New Thermal Neutron Scattering Files for ENDF/B-VI Release 2

R. E. MacFarlane 


\section{CONTENTS}

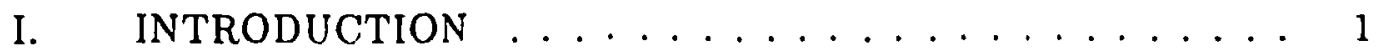

II. THE LEAPR MODULE OF NJOY $\ldots \ldots \ldots \ldots \ldots \ldots$

A. Theory ........................ 4

B. LEAPR Input Instructions $\ldots \ldots \ldots \ldots$

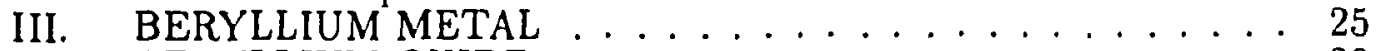

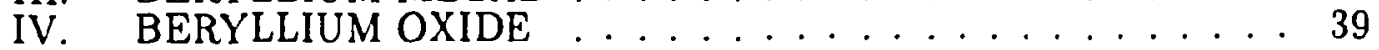

V. GRAPHITE .................... 51

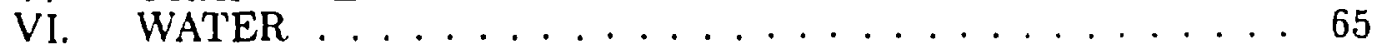

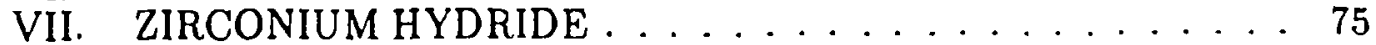

VIII. SOLID METHANE . . . . . . . . . . . . . . . . 95

IX. LIQUID METHANE . . . . . . . . . . . . 101

$\mathrm{X}$ LIQUID HYDROGEN $\ldots \ldots \ldots \ldots \ldots \ldots \ldots \ldots$

XI. LIQUID DEUTERIUM . . . . . . . . . . . . . . . 121

XII. ACKNOWLEDGMENTS . . . . . . . . . . . . 133

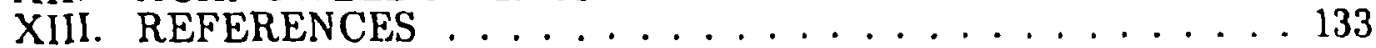




\title{
NEW THERMAL NEUTRON SCATTERING FILES FOR ENDF/B-VI RELEASE 2
}

by

\author{
R. E. MacFarlane
}

\begin{abstract}
At thermal neutron energies, the binding of the scattering nucleus in a solid, liq:id, or gas affects the cross section and the distribution of secondary neutrons. These effects are described in the thermal sublibrary of Version VI of the Evaluated Nuclear Data Files (ENDF /BVI) using the File 7 format. In the original release of the ENDF/B-VI library, the data in File 7 were obtained by converting the thermal scattering evaluations of ENDF/B-III to the ENDF-6 format. These original evaluations were prepared at General Atomics (GA) in the late sixties, and they suffer from accuracy limitations imposed by the computers of the day. This report describes new evaluations for six of the thermal moderator materials and six new cold moderator materials. The calculations were made with the LEAPR module of NJOY, which uses methods based on the British code LEAP, together with the original GA physics models, to obtain new ENDF files that are accurate over a wider range of energy and momentum transfer than the existing files. The new macerials are $\mathrm{H}$ in $\mathrm{H}_{2} \mathrm{O}, \mathrm{Re}$ metal, $\mathrm{Be}$ in $\mathrm{BeO}, \mathrm{C}$ in graphite, $\mathrm{H}$ in $\mathrm{ZrH}, \mathrm{Zr}$ in $\mathrm{ZrH}$, liquid ortho-hydrogen, liquid para-hydrogen, liquid ortho-deuterium, liquid para-deuterium liquid methane, and solid methane.
\end{abstract}

\section{INTRODUCTION}

A good understanding of the scattering of thermal neutrons (that is, neutrons with energies below about $4 \mathrm{eV}$ ) is important for the design of nuclear reactors, spent fuel storage and shipping systems, fuel processing lines, and radiation shielding. In this energy range, the scattering is affected by the binding of the scattering nucleus in the solid, liquid, or gas moderator material. The neutron 
can give up energy to excitations in the material, or it can gain energy. These effects change the reaction cross section, and they modify the energy and angle distribution of the scattered neutrons.

All versions of the US-standard Evaluated Nuclear Data Files (ENDF) since version III have contained evaluations for thermal neutron scattering based on work done at General Atomics (GA) in the sixties. ${ }^{1}$ Because of the limited computer assets available in those days, the calculations were limited to neutron energy transfers of about $1 \mathrm{eV}$, and some numerical problems occured even under these limitations. Modern reactor snalysis codes use thermal scattering methods up to energics as high as $4 \mathrm{eV}$, and the existing data are not adequate to prepare appropriate libraries for them. For some time, we have wanted to produce extended evaluations that remove these limits.

When the ENDF-6 format, which is used for the ENDF/B-VI library, was designed, we included a number of extensions and improvements to the so-called "File 7 Format." ${ }^{2}$ At that time, it was not possible to prepare new evaluations to match the new format, and we simply converted the old evaluations to the new format. The only significant changes made involved renormalizing the basic cross sections to match the low-energy cross sections from ENDF/B-VI. This work was done here at Los Alamos, but we didn't do a very good job with the component of scattering called "coherent elastic," (which shows up for $\mathrm{Be}, \mathrm{BeO}$, and graphite) in the energy range between .1 and $1 \mathrm{eV}$. The "incoherent elastic" component for $\mathrm{Zr}$ in $\mathrm{ZrH}$ is also incorrecl. There has been a strong incentive to correct these problems for some time.

Therefore, to fix the numerical problems, to extend the energy-transfer range of the evaluations, and to correct the elastic scattering problem, we decided to prepare new evaluations for the key moderator materials. This was made possible by the existence of a new module for the NJOY Nuclear Data Processing System ${ }^{3}$ called LEAPR. The LEAPR module is based on the British code LEAP+ADDELT originally written by McLatchie at Harwell in 1962 then implemented by Butland at Winfrith, ${ }^{5}$ and finally modified to work better for cold moderators as part of the Ph.D. Thesis of D. J. Picton, now at the University of Birmingham. We got involved with LEAP at the instigation of Gary Russell of the Lcs Alamos Neutron Scattering Experiment facility (LANSCE), who needed results for cold moderators like liquid hydrogen, liquid deuterium, liquid methane, and solid methane, to use in the design of cold-neutron sources for his facility. 7, 8 Picton kindly provided his version of LEAP to Russell. After work- 
ing with the code and theory for a while, we realized that the phonon expansion method used by LEAP had the capability to compute scattering through larger energy transfers than did the GASKET code ${ }^{9}$ used by GA. Based on this capability, we decided to integrate the core of the LEAP method into NJOY and to add on the other capabilities needed to produce ENDF-6 files for important moderator materials. The first part of this report describes the basic methods used in this new module and gives its input instructions.

Once the LEAPR module was complete, it was used to recompute the thermal scattering files for the most important moderator materials from ENDF/B-VI. The physics models were left the same as in the original GA work. This report contains sections describing the model, the calculated results, and the differences between the old ENDF/B-IV.0 evaluations and the new evaluations for each moderator system. We have also included sections describing new evaluations for several materials useful as moderators for cold neutron sources. The resulting thermal sublibrary files have been submitted to the Cross Section Evaluation Working Group (CSEWG) for inclusion in Release 2 of the ENDF/B-VI library.

\section{THE LEAPR MODULE OF NJOY}

This module is used to prepare the scattering law $S(\alpha, \beta)$ and related quantities, which describe thermal scattering from bound moderators, in the ENDF-6 format used by the THERMK module of NJOY. The original ENDF thermal scattering data ${ }^{1}$ were prepared by by GA using the GASKET code. ${ }^{9}$ This code has difficulty working with the very large energy and momentum transfers encountered for large incident energy or very low temperatures.

The code that Dr. Picton provided was modified extensively to fit better into the NJOY environment and to take advantage of modern large computers. This involved massive rearrangement of storage and routines, updating for FORTRAN-77, and extensive editing of the comment cards. The original Edgewood expansion and short collision time (SCT) treatments were removed in favor of using more terms in the phonon expansion and using the simple ENDF SCT formula. ${ }^{2}$ In addition, output in ENDF-6 format ${ }^{2}$ was provided, the capability to include either coherent or incoherent elastic scattering was added, a major speedup for the diffusion calculation was provided, and a capability to produce a mixed scattering law for materials like $\mathrm{BeO}$ and benzine was developed. In order to improve the numerics on short-word computers, the code was changed to use 
the asymmetric scattering function, the normalization scheme for the phonon expansion was revised, and the discrete-oscillator calculation was rebuilt to take better advantage of the convolution properties of the delta function and to use better Bessel functions. A complete discussion of the theory used in the code is given below.

\section{A. Theory}

The follnwing discussion of the theory used in the the code is based on the original British documentation and the presentation in a standard reference. ${ }^{10}$

1. Coherent and Incoherent Scattering. In practice, the scattering of neutrons from a system of $\mathrm{N}$ particles with a random distribution of spins or isotope types can be expressed as the sum of a coherent part and an incoherent part. The coherent scattering includes the effects from waves that are able to interfere with each other, and the incoherent part depends on a simple sum of noninterfering waves from all the $\mathrm{N}$ particles. (The spin correlations in ortho and para hydrogen violate the assumption of randomness, so liquid hydrogen does not fit into the model described here. A method for treating them will be described below.) The cross sections for coherent and incoherent scattering can be considered to be characteristic properties of the materials. As examples, the scattering from hydrogen is almost completely incuherent, and the scattering from carbon and oxygen is almost completely coherent.

Furthermore, the coherent and incoherent scattering include both elastic and inelastic parts. The elastic scattering takes place with no energy change. It should not be confused with the elastic scattering from a single particle that is familiar for higher neutron energies where the neutron loses energy; thermal elastic scattering can be considered to be scattering from the entire lattice; thus the effective mass of the target is very large, and the neutron does not lose energy in the scattering process. Thermal inelastic scattering results in an energy loss (gain) for the neutron with a corresponding excitation (deexcitation) of the target. The excitation may correspond to the production of one or more phonons in a crystalline material, to the production of rotations or vibrations in molecules, or to the initiation of atomic or molecular recoil motions in a liquid or gas.

In addition, the coherent in elastic part of the scattering contains both inter. ference effects between waves scattered by different particles and direct terms. 
It turns out tiac the direct part for gases, liquids, and solids consisting of randomly oriented crystallites has approximately the same form as the incoherent term. The interference is usually neglected.

Therefore, we can usually divide the thermal scattering cross section into three different parts:

- Coherent eiastic. Important for crystalline solids like graphite or beryllium.

- Incoherent elastic. Important for hydrogenous solids like solid methane, polyethylene, and zirconium hydride.

- Inelastic. Important for all materials (this category includes both incoherent and coherent inelastic).

The absence of interference in incoherent scattering and the neglect of interference in coherent inelastic scattering allow us to construct thermal scattering laws for "hydrogen in water," "hydrogen in solid methane," or "oxygen in beryllium oxide." However, this simplification is not possible in general for coherent elastic scattering in materials with more that one type of atom in the unit cell; for coherent elastic scaitering, beryllium oxide must be considered as a unit.

2. Inelastic Scattering. It is shown in the standard references ${ }^{10}$ that the double differential scattering cross section for thermal neutrons for gases, liquicis, or solids consisting of randomly ordered microcrystals can be written as

$$
\sigma\left(E \rightarrow E^{\prime}, \mu\right)=\frac{\sigma_{b}}{2 k T} \sqrt{\frac{E^{\prime}}{E}} \mathcal{S}(\alpha, \beta),
$$

where $E$ and $E^{\prime}$ are the incident and secondary neutron einergies in the laborat.ory system, $\mu$ is the cosine of the scattering angie in the laboratory, $\sigma_{b}$ is the characteristic bound scattering cross section for the material, $k T$ is the thermal energy in $\mathrm{eV}$, and $\mathcal{S}$ (script $\mathrm{S}$ ) is the asymmetric form of the scattering law. The scattering law depends on only two variables: the momentum transfer

$$
\alpha=\frac{E^{\prime}+E-2 \sqrt{E^{\prime} E} \mu}{A k T},
$$

where $A$ is the ratio of the scatterer mass to the neutron mass, and the energy transfer

$$
\beta=\frac{E^{\prime}-E}{k T} .
$$


Note that $\beta$ is positive for energy gain and negative for energy loss. Working in the i...erent approximation and the Gaussian approximation, the scattering law call be written

$$
\mathcal{S}(\alpha, \beta)=\frac{1}{2 \pi} \int_{-\infty}^{\infty} e^{i \beta \hat{t}} e^{-\gamma(\hat{t})} d \hat{t}
$$

where $\hat{t}$ is time measured in units of $\hbar /(k T)$ seconds. The function $\gamma(\hat{t})$ is given by

$$
\gamma(\hat{t})=\alpha \int_{-\infty}^{\infty} P(\beta)\left[1-\mathrm{e}^{-i \beta \hat{t}}\right] \mathrm{e}^{-\beta / 2} d \beta
$$

where

$$
P(\beta)=\frac{\rho(\beta)}{2 \beta \sinh (\beta / 2)},
$$

and where $\rho(\beta)$ is the frequency spectrum of excitations in the system expressed as a function of $\beta$. The spectrum must be normalized as follows:

$$
\int_{0}^{\infty} \rho(\beta) d \beta=1
$$

The function $P(\beta)$ defined by Eq.(6) is used directly in LEAPR, and $\rho(\beta)=$ $\rho(\epsilon / k t)$ is given as a function of $\epsilon$ in $\mathrm{eV}$. For systems in thermal equilibrium, there is a relationship between upscatter and downscatler called "detail balance" that is a consequence of microscopic reversibility. It requires that

$$
\mathcal{S}(\alpha, \beta)=\mathrm{e}^{-\beta} \mathcal{S}(\alpha,-\beta) \text {. }
$$

Liquid hydrogen and deuterium violate this condition, as will be described below. In addition, the scattering law satisfies two other important constraints, namely, normalization

$$
\int_{-\infty}^{\infty} \mathcal{S}(\alpha, \beta) d \beta=1
$$

and the sum rule

$$
\int_{-\infty}^{\infty} \beta \mathcal{S}(\alpha, \beta) d \beta=-\alpha \text {. }
$$

Actually, ENDF works with the so-called "symmetric" $S(\alpha, \beta)$,

$$
S(\alpha, \beta)=\mathrm{e}^{\beta / 2} \mathcal{S}(\alpha, \beta),
$$

which (except for liquid hydrogen or deuterium) satisfies the condition

$$
S(\alpha, \beta)=S(\alpha,-\beta) .
$$


Note that $\mathcal{S}(\alpha,-\beta)$ for positive $\beta$ describes the downscatter side of the function, and because it is basically proportional to the cross section, it can be represented by reasonable numbers (say $10^{-8}$ to 1 ) for all $\beta$. The symmetric $S(\alpha, \beta)$, on the other hand, can easily be smaller than $\mathcal{S}(\alpha,-\beta)$ by factors like $\mathrm{e}^{-\beta / 2} \sim \mathrm{e}^{-80} \sim$ $10^{-35}$, which can cause trouble on short-word machines. This kind of numerical problem is even more severe for cold moderators, where dynamic ranges on the order of $10^{100}$ occur for $S(\alpha, \beta)$. (The user will have to use some caution reading this report, because the typographic symbols for $\mathrm{S}$ and script-S are very similar.) By worling with $\mathcal{S}(\alpha,-\beta)$, LEAPR can do all of its calculations using singleprecision variables, even on a short-word machine.

The next step is to decompose the frequency spectrum into a sum of simple spectra

$$
\rho(\beta)=\sum_{j=1}^{K} \rho_{j}(\beta),
$$

where the following possibilities are allowed:

$$
\begin{aligned}
& \rho_{j}(\beta)=w_{j} \delta\left(\beta_{j}\right) \text { discrete oscillator, } \\
& \rho_{j}(\beta)=\rho_{s}(\beta) \quad \text { solid-type spectrum, and } \\
& \rho_{j}(\beta)=\rho_{t}(\beta) \quad \text { translational spectrum. }
\end{aligned}
$$

The solid-type spectrum must vary as $\beta^{2}$ as $\beta$ goes to zero, and it must integrate to $w_{\mathbf{s}}$, the weight for the solid-type law. The translational spectrum can be either a free-gas law or a diffusion-type spectrum represented with the approximation of Egelstaff and Schofield that will be discussed later. In either case, the spectrum must integrate to $w_{t}$, the translational weight. The sum of all the weights of the partial spectra must equal 1. Defining $\gamma_{j}(\hat{t})$ to be the value of $\gamma$ appropriate for $\rho_{j}$, and $\mathcal{S}_{j}$ to be the corresponding partial scattering law and using the convolution theorem for Fourier transforms lead to a recursive formula for the scattering law:

$$
\mathcal{S}(\alpha, \beta)=\mathcal{S}^{(K)}(\alpha, \beta)
$$

where

$$
\begin{aligned}
\mathcal{S}^{(J)}(\alpha, \beta) & =\frac{1}{2 \pi} \int_{-\infty}^{\infty} \mathrm{e}^{i \beta \hat{t}} \prod_{j=1}^{J} \mathrm{e}^{-\gamma_{j}(\hat{t})} d \hat{t} \\
& =\int_{-\infty}^{\infty} \mathcal{S}_{J}\left(\alpha, \beta^{\prime}\right) \mathcal{S}^{(J-1)}\left(\alpha, \beta-\beta^{\prime}\right) d \beta^{\prime}
\end{aligned}
$$


As an example of the use of this recursive procedure, consider a case where the desired frequency spectrum is a combination of $\rho_{s}$ and two discrete oscillators. First, calculate $\mathcal{S}^{(1)}=\mathcal{S}_{1}$ using $\rho_{9}$. Then calculate $\mathcal{S}_{2}$ using $\rho\left(\beta_{1}\right)$, the distribution for the first discrete oscillator, and convolve $S_{2}$ with $\mathcal{S}^{(1)}$ to obtain $\mathcal{S}^{(2)}$, the composite scattering law for the first two partial distributions. Repeat the process with the second discrete oscillator to obtain $\mathcal{S}^{(3)}$, which is equal to $\mathcal{S}(\alpha, \beta)$ for the full distribution.

3. The Phonon Expansion. Consider first $\gamma_{s}(\hat{t})$, the Gaussian function for solid-type frequency spectra. Expanding the time-dependent part of the exponential gives

$$
\mathrm{e}^{-\gamma_{s}(\hat{t})}=\mathrm{e}^{-\alpha \lambda_{s}} \sum_{n=0}^{\infty} \frac{1}{n !}\left[\alpha \int_{-\infty}^{\infty} P_{s}(\beta) \mathrm{e}^{-\beta / 2} \mathrm{e}^{-i \beta \hat{t}} \mathrm{~d} \beta\right]^{n}
$$

where $\lambda_{s}$ is the Debye-Waller coefficient

$$
\lambda_{s}=\int_{-\infty}^{\infty} P_{s}(\beta) \mathrm{e}^{-\beta / 2} d \beta
$$

The scattering function becomes

$$
\begin{aligned}
\mathcal{S}_{s}(\alpha, \beta)= & \mathrm{e}^{-\alpha \lambda_{s}} \sum_{n=0}^{\infty} \frac{1}{n !} \alpha^{n} \\
& \times \frac{1}{2 \pi} \int_{-\infty}^{\infty} \mathrm{e}^{i \beta \hat{t}}\left[\int_{-\infty}^{\infty} P_{s}\left(\beta^{\prime}\right) \mathrm{e}^{-\beta^{\prime} / 2} \mathrm{e}^{-i \beta^{\prime} \hat{t}} d \beta^{\prime}\right]^{n} d \hat{t}
\end{aligned}
$$

For convenience, define the quantity in the second line of this equation to be $\lambda_{s}^{n} \mathcal{T}_{n}(\beta)$. Then clearly,

$$
\mathcal{S}_{s}(\alpha, \beta)=\mathrm{e}^{-\alpha \lambda_{s}} \sum_{n=0}^{\infty} \frac{1}{n !}\left[\alpha \lambda_{s}\right]^{n} \mathcal{T}_{n}(\beta)
$$

where

$$
\mathcal{T}_{0}(\beta)=\frac{1}{2 \pi} \int_{-\infty}^{\infty} \mathrm{e}^{i \beta \hat{t}} d \hat{t}=\delta(\beta)
$$

and

$$
\mathcal{T}_{1}(\beta)=\int_{-\infty}^{\infty} \frac{P_{s}\left(\beta^{\prime}\right) \mathrm{e}^{-\beta^{\prime} / 2}}{\lambda_{s}}\left\{\frac{1}{2 \pi} \int_{-\infty}^{\infty} \mathrm{e}^{i\left(\beta-\beta^{\prime}\right) \hat{t}} d \hat{t}\right\} d \beta^{\prime}=\frac{P_{s}(\beta) \mathrm{e}^{-\beta / 2}}{\lambda_{s}}
$$


In general,

$$
\mathcal{T}_{n}(\beta)=\int_{-\infty}^{\infty} \mathcal{T}_{1}\left(\beta^{\prime}\right) \mathcal{T}_{n-1}\left(\beta-\beta^{\prime}\right) d \beta^{\prime}
$$

The script-T functions obey the relationship $\mathcal{T}_{n}(\beta)=\mathrm{e}^{-\beta} \mathcal{T}_{n}(-\beta)$. In addition, each of the $\mathcal{T}_{n}$ functions obeys the following normalization condition:

$$
\int_{-\infty}^{\infty} \mathcal{T}_{n}(\beta) d \beta=1
$$

It guarantees that Eq.(9) will be satisfied by the sum in Eq.(22). In LEAPR, the $\mathcal{T}_{n}(-\beta)$ functions are precomputed on the input $\beta$ grid for $n$ up to some specified maximum value, typically 100 . It is then easy to compute the smooth part of $\mathcal{S}_{s}(\alpha,-\beta)$ for any sufficiently small value of $\alpha$ using Eq.(22). The corresponding values of $\mathcal{S}_{s}(\alpha, \beta)$ can then be obtained by multiplying by $\mathrm{e}^{-\beta}$. The delta function arising from the "zero-phonon" term is carried forward separately. The normalization in Eq.(22) has better numerical properties than the one used in LEAP.

4. The Short-Collision-Time Approximation. For larger values of $\alpha$, the expansion of Eq.(22) requires too many terms. LEAPR uses the simple SCT approximation from ENDF to extend the solid-type scattering law.

$$
\mathcal{S}_{s}(\alpha,-\beta)=\frac{1}{\sqrt{4 \pi w_{s} \alpha \bar{T}_{s} / T}} \exp \left[-\frac{\left(w_{s} \alpha-\beta\right)^{2}}{w_{s} \alpha \bar{T}_{s} / T}\right]
$$

and

$$
\mathcal{S}_{s}(\alpha, \beta)=\mathrm{e}^{-\beta} \mathcal{S}_{s}(\alpha,-\beta),
$$

where $\beta$ is positive, and where the effective temperature is given by

$$
\bar{T}_{s}=\frac{T}{2 w_{s}} \int_{-\infty}^{\infty} \beta^{2} P_{s}(\beta) \mathrm{e}^{-\beta} d \beta
$$

As above, $w_{\mathrm{s}}$ is the weight for the solid-type spectrum.

5. Diffusion and Free-Gas Translation. The neutron scattering from many important liquids, including water and liquid methane, can be represented using a solid-type spectrum of rotational and vibrational modes combined with a diffusion term. Egelstaff and Schofield have proposed an especially simple model 
for diffusion called the "effective width model". It has the advantage of having analytic forms for both $S(\alpha, \beta)$ and the associated frequency spectrum $\rho(\beta)$ :

$$
\begin{aligned}
\mathcal{S}_{t}(\alpha, \beta) & =\frac{2 c w_{t} \alpha}{\pi} \exp \left[2 c^{2} w_{t} \alpha-\beta / 2\right] \\
& \times \frac{\sqrt{c^{2}+.25}}{\sqrt{\beta^{2}+4 c^{2} w_{t}^{2} \alpha^{2}}} K_{1}\left\{\sqrt{c^{2}+.25} \sqrt{\beta^{2}+4 c^{2} w_{t}^{2} \alpha^{2}}\right\}
\end{aligned}
$$

and

$$
\rho(\beta)=w_{t} \frac{4 c}{\pi \beta} \sqrt{c^{2}+.25} \sinh (\beta / 2) K_{1}\left\{\sqrt{c^{2}+.25} \beta\right\} .
$$

In these equations, $K_{1}(x)$ is a modified Bessel function of the second kind, and the translational weight $w_{t}$ and the diffusion constant $c$ are provided as inputs.

An alternative for the translational part of the distribution is ite free-gas law. It is clearly appropriate for a gas of molecules, but it has also been used to represent the translational component for liquid moderators like water. ${ }^{i}$ The scattering law is given by

$$
\mathcal{S}_{t}(\alpha,-\beta)=\frac{1}{\sqrt{4 \pi w_{t} \alpha}} \exp \left[-\frac{\left(w_{t} \alpha-\beta\right)^{2}}{4 w_{t} \alpha}\right],
$$

and

$$
\mathcal{S}_{t}(\alpha, \beta)=\mathrm{e}^{-\beta} \mathcal{S}_{t}(\alpha,-\beta),
$$

with $\beta$ positive. The free-gas law is used in LEAPR if the diffusion coefficient $c$ is input as zero.

In LEAPR, $\mathcal{S}_{s}(\alpha, \beta)$, the scattering law for the solid-type modes, is calculated using the phonon expansion as described above. The translational contribution $\mathcal{S}_{t}(\alpha, \beta)$ is then calculated using one of the formulas above on a $\beta$ grid chosen to represent its shape fairly well. The combined scattering law is then obtained by convolution as follows:

$$
\mathcal{S}(\alpha, \beta)=\mathcal{S}_{t}(\alpha, \beta) \mathrm{e}^{-\alpha \lambda_{s}}+\int_{-\infty}^{\infty} \mathcal{S}_{t}\left(\alpha, \beta^{\prime}\right) \mathcal{S}_{\mathfrak{s}}\left(\alpha, \beta-\beta^{\prime}\right) d \beta^{\prime}
$$

The first term arises from the delta function in Eq.(22), which isn't included in the numerical results for the phonon series calculation. The values for $\mathcal{S}_{t}(\beta)$ and $\mathcal{S}_{s}\left(\beta-\beta^{\prime}\right)$ are obtained from the precomputed functions by interpolation. This makes LEAPR run much faster than LEAP+ADDELT for 'diffusive cases, because the original code did direct recalculations of the solid-type scattering 
law for all the desired values of $\beta-\beta^{\prime}$. It also had to take pains to compute $S_{t}$ on a $\beta$ grid that was commensurate with the input grid. This often resulted in more points for $S_{t}$ than were necessary to obtain useful accuracy for the convolutions.

The effective temperature for a combination of solid-type and translation modes is computed using

$$
\bar{T}_{s}=\frac{w_{t} T+w_{s} \bar{T}_{s}}{w_{t}+w_{s}}
$$

6. Discrete Osciliators. Polyatomic molecules normally contain a number of vibrational modes that can be represented as discrete oscillators. The distribution function for one oscillator is given by $w_{i} \delta\left(\beta_{i}\right)$, where $w_{i}$ is the fractional weight for mode $i$, and $\beta_{i}$ is the energy-transfer parameter computed from the mode's vibrational frequency. The corresponding scattering law is given by

$$
\begin{aligned}
\mathcal{S}_{i}(\alpha, \beta) & =\mathrm{e}^{-\alpha \lambda_{i}} \sum_{n=-\infty}^{\infty} \delta\left(\beta-n \beta_{i}\right) I_{n}\left[\frac{\alpha w_{i}}{\beta_{i} \sinh \left(\beta_{i} / 2\right)}\right] \mathrm{e}^{-n \beta_{i} / 2} \\
& =\sum_{n=-\infty}^{\infty} A_{i n}(\alpha) \delta\left(\beta-n \beta_{i}\right)
\end{aligned}
$$

where

$$
\lambda_{i}=w_{i} \frac{\operatorname{coth}\left(\beta_{i} / 2\right)}{\beta_{i}} .
$$

The combination of a solid-type mode (s) with discrete oscillators (1) and (2) would give

$$
\begin{aligned}
\mathcal{S}^{(0)}(\alpha, \beta) & =\mathcal{S}_{s}(\alpha, \beta), \\
\mathcal{S}^{(1)}(\alpha, \beta) & =\int_{-\infty}^{\infty} \mathcal{S}_{1}\left(\alpha, \beta^{\prime}\right) \mathcal{S}^{(0)}\left(\alpha, \beta-\beta^{\prime}\right) d \beta^{\prime} \\
& =\sum_{n=-\infty}^{\infty} A_{1 n}(\alpha) \mathcal{S}^{(0)}\left(\alpha, \beta-n \beta_{1}\right), \text { and } \\
\mathcal{S}^{(2)}(\alpha, \beta) & =\int_{-\infty}^{\infty} \mathcal{S}_{2}\left(\alpha, \beta^{\prime}\right) \mathcal{S}^{(1)}\left(\alpha, \beta-\beta^{\prime}\right) d \beta^{\prime} \\
& =\sum_{m=-\infty}^{\infty} A_{2 m}(\alpha) \sum_{n=-\infty}^{\infty} A_{1 n}(\alpha) \mathcal{S}^{(0)}\left(\alpha, \beta-n \beta_{1}-m \beta_{2}\right) .
\end{aligned}
$$

This process can be continued through $\mathcal{S}^{(3)}(\alpha, \beta), \mathcal{S}^{(4)}(\alpha, \beta)$, etc., until all the discrete oscillators have been included. The result has the form

$$
\mathcal{S}(\alpha, \beta)=\sum_{k} W_{k}(\alpha) \mathcal{S}_{\mathbf{s}}\left(\alpha, \beta-\beta_{k}\right)
$$


where the $\beta_{k}$ and the associated wcights $W_{k}$ are easily generated recursively using a procedure that throws out small weights at each step. The Debye-Waller $\lambda$ for the combined modes is computed using

$$
\lambda=\lambda_{s}+\sum_{i=1}^{N} \lambda_{i}
$$

The effective temperature for the combined modes is given by

$$
\bar{T}_{s}=w_{t} T+w_{s} \bar{T}_{s}+\sum_{i=1}^{N} w_{i} \frac{\beta_{i}}{2} \operatorname{coth}\left(\frac{\beta_{i}}{2}\right) T .
$$

If the starting-point scattering law $\mathcal{S}^{(0)}$ does not contain a translational contribution (true for hydrogenous solids like polyethylene and frozen methane), it is important to remember to include the effects of the "zero-phonon" term $\exp \left(-\alpha \lambda_{s}\right) \delta(\beta)$. The code does this by adding in triangular peaks with the proper areas and with their apexes at the $\beta$ value closest to the $\beta_{k}$ values. One of these peaks is at $\beta=0$. This peak is not put into the scattering law as a sharp triangle; instead, it is handled as "incoherent elastic" scattering in order to take full advantage of the analytic properties of $\delta(\beta)$.

7. Incoherent Elastic Scattering. In hydrogenous solids, there is an elastic (no energy loss) component of scattering arising from the "zero-phonon," or $n=0$ term, of Eq.(22). In ENDF terminology, this is called the "incrherent elastic" term. Clearly,

$$
S_{\text {iel }}(\alpha, \beta)=\mathrm{e}^{-\alpha \lambda} \delta(\beta) .
$$

The corresponding differential scattering cross section is

$$
\sigma(E, \mu)=\frac{\sigma_{b}}{2} \mathrm{e}^{-2 W E(1-\mu)}
$$

and the integrated cross section is

$$
\sigma(E)=\frac{\sigma_{b}}{2}\left\{\frac{1-\mathrm{e}^{-4 W E}}{2 W E}\right\} .
$$

In these equations, the Debye-Waller coefficient is given by

$$
W=\frac{\lambda}{A k T},
$$


where $\lambda$ is computed from the input frequency spectrum as shown by Eq.(20) and modified by the presence of discrete oscillators (if any) as shown above. LEAPR writes the bound scattering cross section $\sigma_{b}$ and the Debye-Waller coefficient $W$ as a function of temperature into a section of the ENDF-6 output with MF=7 and $\mathrm{MT}=2$.

8. Coherent Elastic Scattering. In solids consisting of coherent scatterersfor example, graphite--the zero-phonon term leads to interference scattering from the various planes of atoms of the crystals making up the solid. Once again, there is no energy loss, and the ENDF term for the process is "coherent eliastic scattering". The differential scattering cross section is given by

$$
\sigma_{\text {coh }}(E, \mu)=\frac{n_{c}}{E} \sum_{t_{i}<E} f_{i}-4 W E_{i} \delta\left(u-\mu_{i}\right)
$$

where

$$
\mu_{i}=1-E_{i} / E,
$$

and the integrated cross section is given by

$$
\sigma_{\text {coh }}=\frac{\sigma_{c}}{E} \sum_{E_{i}<E} f_{i} \mathrm{e}^{-4 W E_{i}}
$$

In these equations, $\sigma_{c}$ is the effective bound coherent scattering cross section for the material, $W$ is the effective Debye-Waller coefficient, $E_{i}$ are the so-called "Bragg edges", and the $f_{i}$ are related to the crystallographic structure factors.

It can be seen from Eq.(50) that the coherent elastic cross section is zero below the first Bragg Edge, $E_{1}$ (typically about 2 to $5 \mathrm{meV}$ ). It then jumpa sharply to a value determined by $f_{1}$ and the Debye-Waller term. At higher energies, the cross section drops off as $1 / E$ until $E=E_{2}$. It then takes another jump, and resumes its $1 / E$ drop off. The sizes of the steps in the cross section gradually get smaller, and at high energies there is nothing left but an asymptotic $1 / E$ decrease (typically above 1 to $2 \mathrm{eV}$ ). LEAPR stores the quantity $E \sigma_{\text {coh }}(E)$ as a function of energy and temperature in a section of the ENDF-6 output with $\mathrm{MF}=7$ and $\mathrm{MT}=2$. The cross section is easily recovered from this representation by dividing by $E$ (this is done in the THERMR module of NJOY). The angular distribution of scattered neutrons can be calculated by extracting the $f_{i}$ from the steps in $\sigma_{\text {coh }}(E)$ file by subtraction. This process is carried out automatically in the GROUPR module of NJOY. 
The calculation of the $E_{i}$ and $f_{i}$ depends on a knowledge of the crystal structure of the scattering material. The methods used are borrowed from HEXSCAT. ${ }^{11}$ In general, the energies of the Bragg edges are given by

$$
E_{i}=\frac{\hbar^{2} \tau_{i}^{2}}{8 m}
$$

where $\tau_{i}$ is the length of the vectors of one particular "shell" of the reciprocal lattice, and $m$ is the neutron inass. The $f_{i}$ fac"ors for a material containing a single atomic species are given by

$$
f_{i}=\frac{2 \pi r^{2}}{4 m N V} \sum_{\tau_{i}}|F(\tau)|^{2},
$$

where the sum extends over all reciprocal lattice vectors of the given length, and the crystallographic structure factor is given by

$$
|F(\tau)|^{2}=\left|\sum_{j=1}^{N} \mathrm{e}^{i 2 \pi \phi_{j}}\right|^{2}
$$

where $N$ is the number of atoms in the unit cell, $\phi_{j}=\vec{\tau} \cdot \vec{\rho}_{j}$ are the phases for the atoms, and the $\vec{\rho}_{j}$ are their positions. The situation is more complicated for materials containing different atomic species, such as beryllium oxide. In these cases,

$$
\sigma_{c} \mathrm{e}^{-2 W E_{i}} f_{i}=\left|\sum_{j=1}^{N} \sqrt{\sigma_{j}} \mathrm{e}^{-W_{j} E_{i}} \mathrm{e}^{i 2 \pi \phi_{j}}\right|^{2},
$$

where the coherent cross section and Debye-Waller factor can be different for each site in the unit cell. The effective coherent cross section is clearly given by

$$
\sigma_{c}=\sum_{j=1}^{N} \sigma_{j}
$$

Since LEAPR only works with one material at a time, it doesn't have access to different values of $W_{j}$ for the atoms in the unit cell. Therefore, it assumes that either $W_{j} E_{i}$ is small, or the $W_{j}$ doesn't vary much from site to site. This allows it to calculate the $f_{i}$ using

$$
|F|^{2}=\left|\sum_{j=1}^{N} \frac{\sqrt{\sigma_{j}}}{\sqrt{\sigma_{c}}} \mathrm{e}^{-2 \pi \phi_{j}}\right|^{2}
$$


For hexagonal materials, the lattice is described by the two constants $a$ and $c$. The reciprocal lattice vector lengths are given by

$$
\left(\frac{\tau}{2 \pi}\right)=\frac{4}{3 a^{2}}\left(\ell_{1}^{2}+\ell_{2}^{2}+\ell_{1} \ell_{2}\right)+\frac{1}{c^{2}} \ell_{3}^{2}
$$

where $\ell_{1}, \ell_{2}$, and $\ell_{3}$ run over all the positive and negative integers, including zero. The volume of the unit cell is

$$
V=\sqrt{3} a_{2} c / 2 .
$$

For graphite, there are four atoms in the unit cell at positions ${ }^{12}$

$$
(0,0,0),\left(-\frac{1}{3}, \frac{1}{3}, 0\right),\left(-\frac{2}{3},-\frac{1}{3}, \frac{1}{2}\right),\left(-\frac{1}{3}, \frac{1}{3}, \frac{1}{2}\right) .
$$

These positions give the follo:wing phases:

$$
\begin{aligned}
& \phi_{1}=0, \\
& \phi_{2}=\left(-\ell_{1}+\ell_{2}\right) / 3, \\
& \phi_{3}=-(2 / 3) \ell_{1}-(1 / 3) \ell_{2}+(1 / 2) \ell_{3}, \text { and } \\
& \phi_{4}=-(1 / 3) \ell_{1}+(1 / 3) \ell_{2}+(1 / 2) \ell_{3} .
\end{aligned}
$$

The form factor for graphite becomes

$$
|F|^{2}= \begin{cases}6+10 \cos \left[2 \pi\left(\ell_{1}-\ell_{2}\right) / 3\right] & \ell_{3} \text { even } \\ 4 \sin ^{2}\left[\pi\left(\ell_{1}-\ell_{2}\right) / 3\right] & \ell_{3} \text { odd }\end{cases}
$$

For the hexagonal close packed (hcp) structure, which includes beryllium, there are two atoms per unit cell at

$$
(0,0,0),\left(\frac{1}{3}, \frac{2}{3}, \frac{1}{2}\right)
$$

and the form factor for hcp lattices, such as beryllium, becomes

$$
|F|^{2}=2+2 \cos \left[2 \pi\left(2 \ell_{1}+4 \ell_{2}+3 \ell_{3}\right) / 6\right] \text {. }
$$

The beryllium oxide lattice consists of two interpenetrating hcp lattices, one for the beryllium atoms, and one for the oxygen. There are four atoms per unit 
cell with positions

$$
(0,0,0),\left(\frac{1}{3}, \frac{2}{3}, \frac{1}{2}\right),(0,0, u),\left(\frac{1}{3}, \frac{2}{3}, u+\frac{1}{2}\right)
$$

where $u$ is .378 (which is very close to $3 / 8$ ). Using the approximation that the Debye-Waller factor doesn't vary from position to position in the unit cell gives the following expression for the structure factor:

$$
|F|^{2}=\left(2+2 \cos \left[2 \pi\left(2 \ell_{i}+4 \ell_{2}+3 \ell_{3}\right) / 6\right]\right)\left(r_{1}^{2}+2 r_{1} r_{2} \cos \left(3 \pi \ell_{3} / 4\right)+r_{2}^{2}\right)
$$

where $r_{1}^{2}$ and $r_{2}^{2}$ are the bound coherent cross sections for beryllium and oxygen, respectively, and the effective coherent cross section, $\sigma_{c}$, is to be taken as 1 . More formulas for the structure factor can be added to the code when needed.

9. Liquid Hydrogen and Deuterium. Materials containing hydrogen (H) or deuterium (D) molecules violate the assumption that spins are distributed randomly that underlies the incoherent approximation used for Eq.(22), and an explicitly quantum-mechanical formula is required to take account of the correlations between the spins of two atoms in the same molecule. This problem was considered by Young and Koppel. ${ }^{13}$ Changing to our notation, the formulas for the hydrogen molecule (neglecting vibrations) become

$$
\begin{aligned}
\mathcal{S}_{\text {para }}(\alpha, \beta) & =\sum_{J=0,2,4, \ldots} P_{J} \\
& \times \frac{4 \pi}{\sigma_{b}}\left[A_{\text {para }} \sum_{J^{\prime}=0,2,4, \ldots}+B_{\text {para }} \sum_{J^{\prime}=1,3,5, \ldots}\right]\left(2 J^{\prime}+1\right) \\
& \times S_{f}\left(w \alpha, \beta+\beta_{J J^{\prime}}\right) \\
& \times \sum_{\ell=\left|J^{\prime}-J\right|}^{J^{\prime}+J} 4 j_{\ell}^{2}(y) C^{2}\left(J J^{\prime} \ell ; 00\right)
\end{aligned}
$$

and

$$
\begin{aligned}
\mathcal{S}_{\text {ortho }}(\alpha, \beta) & =\sum_{J=1,3,5, \ldots} P_{J} \\
& \times \frac{4 \pi}{\sigma_{b}}\left[A_{\text {ortho }} \sum_{J^{\prime}=0,2,4, \ldots}+B_{\text {ortho }} \sum_{J^{\prime}=1,3,5, \ldots}\right]\left(2 J^{\prime}+1\right) \\
& \times S_{f}\left(w \alpha, \beta+\beta_{J J^{\prime}}\right)
\end{aligned}
$$




$$
\times \sum_{\ell=\left|J^{\prime}-J\right|}^{J^{\prime}+J} 4 j_{\ell}^{2}(y) C^{2}\left(J J^{\prime} \ell ; 00\right)
$$

The coefficents for the even and odd sums are given below.

\begin{tabular}{lcc} 
Type & $A($ even $)$ & $B$ (odd) \\
\hline H para & $a_{c}^{2}$ & $a_{i}^{2}$ \\
H ortho & $a_{c}^{2} / 3$ & $a_{c}^{2}+2 a_{i}^{2} / 3$ \\
D para & $3 a_{i}^{2} / 4$ & $a_{c}^{2}+a_{i}^{2} / 4$ \\
D ortho & $a_{c}^{2}+5 a_{i}^{2} / 8$ & $3 a_{i}^{2} / 8$ \\
\hline
\end{tabular}

Here $a_{c}$ and $a_{i}$ are the coherent and incohereni scattering lengths (note that the characteristic bound cross section $\left.\sigma_{b}=4 \pi\left[a_{c}^{2}+a_{i}^{2}\right]\right), P_{J}$ is the statistical weight factor, $\beta_{J J^{\prime}}=\left(E_{J}^{\prime}-E_{J}\right) / k T$ is the energy transfer for a rotational transition, $j_{\ell}(x)$ is a spherical Bessel function of order $\ell$, and $C\left(J J^{\prime} \ell ; 00\right)$ is a Clebsch-Gordan coefficient. The parameter $y$ is given by $\kappa a / 2=(a / 2) \sqrt{M k T \alpha}$, where $a$ is the interatomic distance in the molecule, and $M$ is the molecular mass. The translational weight $w$ is $1 / 2$ for $\mathrm{H}_{2}$ and $1 / 4$ for $\mathrm{D}_{2}$. The sums over $J^{\prime}$ are treated as operators in order to keep the notation compact.

Young and Koppel assumed that the molecular translations were free, so the equations contain

$$
\mathcal{S}_{f}(\alpha,-\beta)=\frac{1}{\sqrt{4 \pi \alpha}} \exp \left[-\frac{(\alpha-\beta)^{2}}{4 \alpha}\right] \text {, }
$$

and

$$
\mathcal{S}_{f}(\alpha, \beta)=\mathrm{e}^{-\beta} \mathcal{S}_{f}(\alpha,-\beta),
$$

the free-atom scattering function (with $\beta$ positive). Note that $\alpha$ is multiplied by a translational weight of 0.5 or 0.25 when this equation is used in order to make the formula apply to a molecule with mass ratio 2 or 4 , respectively.

These formulas as stated are appropriate for a gas of hydrogen or deuterium molecules. In a liquid, there are two additional effects to be considered: interference between the neutron waves scattered from different molecules, and the fact that the recoil of the hydrogen molecule is not really free. First, we will consider the latter effect. Experiments by Egelstaff, Haywood, and Webb at Harwell ${ }^{24}$ and Schott at Karlsruhe ${ }^{25}$ showed appreciable broadening of the quasi-elastic scattering peak for liquid hydrogen, and both groups ascribed this 
to diffusive effects. Later, Utsuro of Kyoto University constructed a simple analytic model ${ }^{26}$ that included both diffusion and intermolecular vibrations and showed good agreement with experiment. More recently, Keinert and Sax of the University of Stuttgart proposed the model 27 that we follow here.

They suggested that the free translation term in the Young and Koppel formulas be replaced by the superposition of a solid-state like motion and a diffusive law. One can picture a hydrogen molecule bound in a cluster of about 20 molecuies and undergoing vibrations similar to those of a hydrogen molecule in a solid. These clumps then diffuse through the liquid (hindered translations) according to the Egelstaff-Schofield effective width model discussed above. The details of the performance of this model will be discussed further in a subsequent section.

As mentioned earlier, waves scattered from different molecules can also interfere. Intermolecular coherence results when there is a correlation between the positions of nearby molecules. This kind of coherence is described by the "static structure factor" $S(\kappa)$. This quantity can be used in an approximation due to Vineyard 28 as follows:

$$
\frac{d^{2} \sigma}{d \Omega d \epsilon}=\frac{d^{2} \sigma_{\text {coh }}}{d \Omega d \epsilon} S(\kappa)+\frac{d^{2} \sigma_{\text {incoh }}}{d \Omega d \epsilon}
$$

This is equivalent to using Eqs.(66) and (67) with $a_{c}^{2}$ replaced by $S(\kappa) a_{c}^{2}$ in the calculation of the coefficients $A$ and $B$. The effects of this procedure will be shown below.

10. Mixed Moderators. In some cases, thermal evaluations give the scattering for a principal scatterer as bound in a moderator, for example, $\mathrm{H}$ in $\mathrm{H}_{2} \mathrm{O}$, or $\mathrm{Zr}$ in $\mathrm{ZrH}$. The other atoms in the molecule are represented by an analytic law (free-gas $\mathrm{O}$ ), or by another detailed scattering law ( $\mathrm{H}$ in $\mathrm{ZrH}$ ). In other cases, the scattering from the entire molecule is represented in one file. Examples from ENDF are $\mathrm{BeO}$ and methane. The molecular scattering is renormalized to be used with the principal scatterer (Be or $\mathrm{H}$ for these cases); the secondary scatterer is assumed to have zero scattering in the thermal range. 
Taking $\mathrm{BeO}$ as an example, the thermal cross section can be represented as follows:

$$
\begin{aligned}
\sigma_{\mathrm{BeO}}= & \frac{\sigma_{b, \mathrm{Be}}}{2 k T} \sqrt{\frac{E^{\prime}}{E}} \mathrm{e}^{-\beta / 2} \\
& \left\{S_{\mathrm{Be}}\left(\alpha_{\mathrm{Be}}, \beta\right)+\frac{\sigma_{b, \mathrm{O}}}{\sigma_{b, \mathrm{Be}}} S_{\mathrm{O}}\left(\frac{A_{\mathrm{Be}}}{A_{\mathrm{O}}} \alpha_{\mathrm{Be}}, \beta\right)\right\},
\end{aligned}
$$

where $\alpha_{\mathrm{Be}}$ stands for $\alpha$ computed with the atomic mass ratio for $\mathrm{Be}, A_{\mathrm{Be}}$. In practice, LEAPR first computes $S_{\mathrm{Be}}$ using the input $\alpha$ grid, and then it computes $S_{\mathrm{O}}$ on a new $\alpha$ grid obtained by transforming the input grid with the indicated mass ratio. The two parts can now be added up by weighting with the indicated ratio of the bound cross sections. The method for preparing a mixed $S(\alpha, \beta)$ for $\mathrm{BeO}$ is shown in detail below.

\section{B. LEAPR Input Instructions}

The following listing of input instructions was copied from the comment cards at the beginning of the LEAPR module. It is always a good idea to check the current source file in case there have been changes.

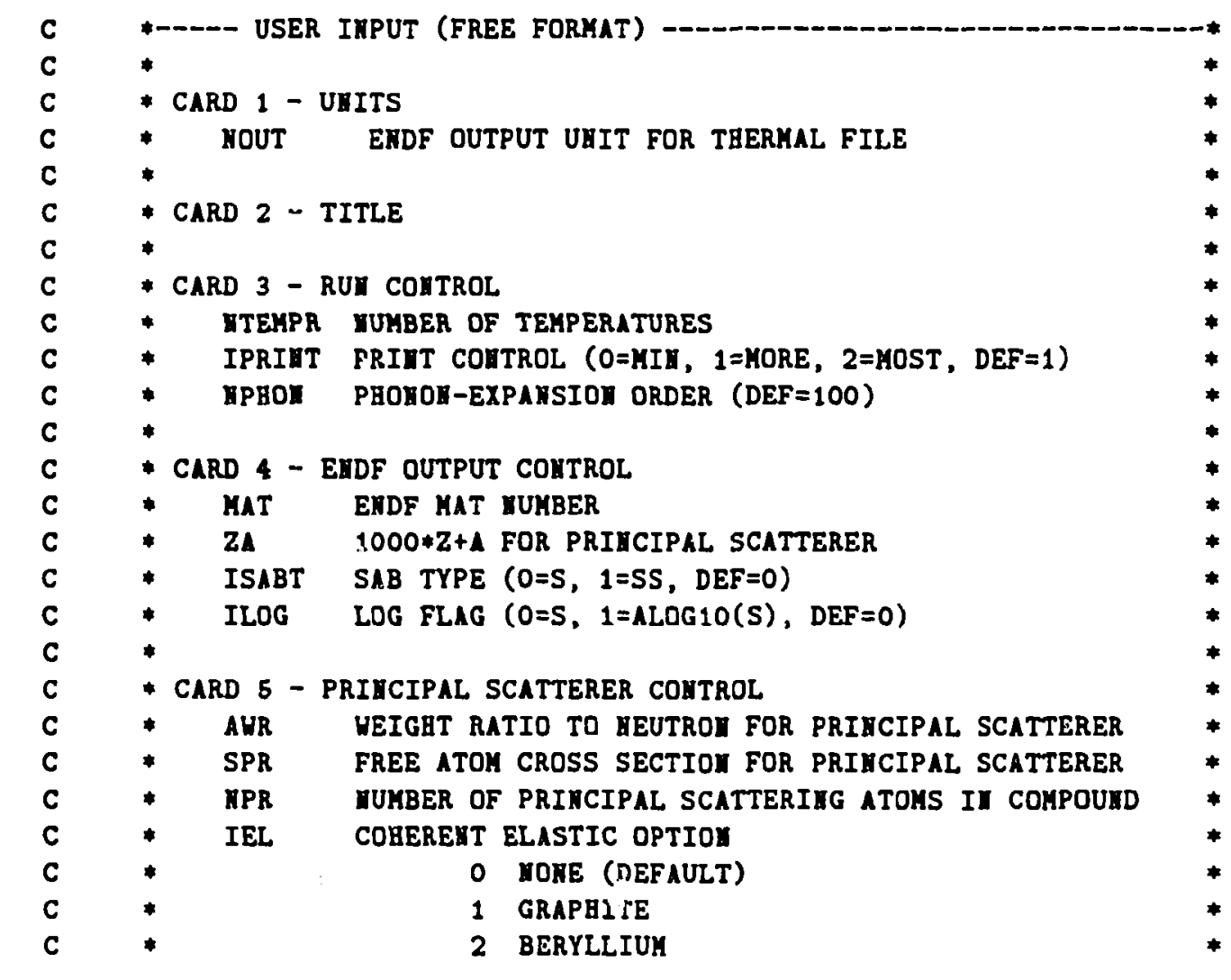




\begin{tabular}{|c|c|}
\hline C & 3 BERYLLIUK OXIDE \\
\hline C & COLD BYDROGER OPTION $(D E F=0)$ \\
\hline C & ORTEO BYDROGEI \\
\hline C & PARA BYDROGEM \\
\hline C & 3 OTHO DEUTERIUM \\
\hline C & 4 PARA DEUTERIUY \\
\hline C & $\star$ \\
\hline C & * CARD 6 - SECOIDARY SCATTERER CONTROL \\
\hline C & MUMBER OF SECOMDARY SCATTERERS (O OR 1) \\
\hline C & SECORDARY SCATTERER TYPE \\
\hline C & (0=SCT DXLY, 1=FREE, 2=DIFFUSIOR) \\
\hline C & HEIGET RATIO TO DEUTROI FOR SECOHDARY SCATTERER \\
\hline C & FREE ATOMS CROSS SECTIOH FOR SECOHDARY SCATTERER \\
\hline C & MUMBER OF ATOMS OF TEIS TYPE II TBE COHPOUAD \\
\hline C & * \\
\hline C & - CARD 7 - ALPBA, BETA COHTROL \\
\hline C & MALPEA IUABER OF ALPBA VALUES \\
\hline C & MBETA HUMBER OF BETA VALUES \\
\hline C & IF LAT.EQ.1, ALPBA AED BETA VALUES ARE SCALED \\
\hline C & BY $.0253 / \mathrm{TEV}$, WEERE TEV IS TEMP II EV. (DEF=0) \\
\hline C & $\star$ \\
\hline C & * CARD 8 - ALPBA VALUES (IRCREASIMG ORDER) \\
\hline C & * CARD 9 - BETA VALUES (IMCREASIMG ORDER) \\
\hline C & * \\
\hline C & * SCATTERER LOOP, DO TEMPERATURE LOOP FOR PRIMCIPAL SCATTERER. \\
\hline C & * REPEAT FOR SECONDARY SCATTERER (IF AIY) IF B7=0. \\
\hline C & * \\
\hline C & * TEMPERATURE LOOP, REPEAT CARDS 10 TO 18 FOR EACB TEMPERATURE \\
\hline C & * \\
\hline C & CARD 10 - TEMPERATURE (K) \\
\hline C & A MEgATIVE VALUE MEAMS SKIP CARDS 11 TO 18 , \\
\hline C & TBEREBY USIMG PREVIOUS PARAMETERS FOR THIS TEMP. \\
\hline C & * \\
\hline C & CARD 11 -- CONTIEUOUS DISTRIBUTIDA COATROL \\
\hline C & DELTA INTERVAL IN EV \\
\hline C & RI BUHBER OF POIHTS \\
\hline C & * \\
\hline C & CARD 12 -- RBO(EGERGY) (ORDER OF IMCREASIMG EV) \\
\hline C & * \\
\hline C & CARD 13 - CORTILUOUS DISTRIBUTIOI PARAKETERS \\
\hline C & TRARSLATIORAL MEIGBT \\
\hline C & DIFFUSIOI COKSTART (ZERO FOR FREE GAS) \\
\hline C & MORHALIZATIOA FOR COHTIMUOUS PART \\
\hline C & * \\
\hline C & CARD 14 - DISCRETE OSCILLATOR CORTROL \\
\hline C & MUKBER OF DISCRETE OSCILLATORS \\
\hline C & * \\
\hline C & CARD 15 - OSCILLATOR EHERGIES (EV) \\
\hline C & CARD 16 - OSCILLATOR WEIGBTS (SUM TO 1.-TBETA-TWT) \\
\hline C & * \\
\hline C & CARD 17 - PAIR CORRELATIOA COHTROL (BCOLD.GT.O OHLY) \\
\hline C & HUMBER OF KAPPA VALUES \\
\hline C & KAPPA IBCREMENT (INV. AHGSTROMS) \\
\hline C & CARD 18 SKAPPA VALUES IR IMCREASIRG ORDER (IMV. ABG.) \\
\hline
\end{tabular}




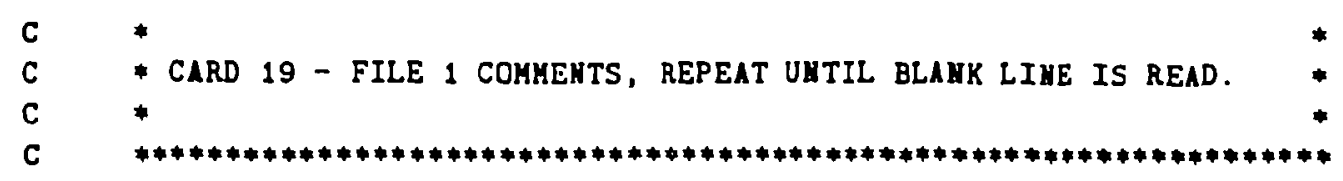

Card 1 is the standard NJOY units card. NOUT should be a number from 20 up, and it can be either positive (ASCII) or negative (blocked binary).

The title card is just used to label the input deck and the output listing for the user's convenience. The title string does not go into the output ENDF file.

Card 3 contains global parameters that coritrol the run. The meaning of NTEMPR is clear. The amount of information printed on the output listing is controiled by IFRINT. The default is 1 (a mid-size listing). If you suspect problems, you can turn on the long listing to see more details. NPHON gives the order of the phonon expansion. Usually a fairly large number (such as 100) is suitable. If it is too small, the SCT approximation will be used to excess.

The ENDF MAT number on Card 4 should be one of the numbers listed in Appendix C of ENDF-102. ${ }^{2}$ For the user's convenience, the numbers currently being used are given in the following table:

Table 1: Material (MAT) and Reaction (MT) Numbers Used in ENDF Thermal Evaluations and NJOY Processing

\begin{tabular}{lcclcc} 
Compound & MAT & MT & Compound & MAT & MT \\
\hline Water & 1 & 222 & $\mathrm{BeO}$ & 27 & 233,234 \\
Para Hydrogen & 2 & & Graphite & 31 & 229,230 \\
Ortho Hydrogen & 3 & & Polyethylene & 37 & 223,224 \\
H in ZrH & 7 & 225,226 & Benzine & 40 & 227 \\
Heavy Water & 11 & 228 & $\mathrm{Zr} \mathrm{in} \mathrm{ZrH}$ & 58 & 235,236 \\
Para Deuterium & 12 & & $\mathrm{UO}_{2}$ & 75 & \\
Ortho Deuterium & 13 & & $\mathrm{UC}$ & 76 & \\
Be & 26 & 231,232 & & & \\
\hline
\end{tabular}

The table also gives the non-standard MT numbers used to label these reactions in NJOY THERMR, GROUPR, and MATXSR runs. The appropriate value to be used for ZA is fairly obvious. The parameter ISYM controls whether the output ENDF tape contains the symmetric $S(\alpha, \beta)$ or the asymmetric $S(\alpha, \beta)$. The 
second option gives much better numerics, especially on short-word machines, but it is not sanctioned by the ENDF-6 format. ILOG controls whether the output file contains $S$ (or $S$ ) or $\log _{10} S$. Giving the $\log$ of $S$ is the ENDF-sanctioned way of handling very small numbers it. File 7.

The "principal scatterer" may be hard to select for some compounds. For water, it is $\mathrm{H}$. But for $\mathrm{ZrH}_{\mathrm{r}}$, it would be $\mathrm{H}$ for $\mathrm{MAT}=$ ? and $\mathrm{Z}_{\mathrm{r}}$ for $\mathrm{MAT}=58$. For mixed moderators, such as $\mathrm{BeO}$, it is usually the lighter material. The value of SPR should be chosen by looking at the low-energy limit for $M F=3, M T=2$ (elastic scattering) on the neutron file to be used with the new evaluation. The value for NPR would be 2 for $\mathrm{H}_{2} \mathrm{O}$, or 1 for $\mathrm{BeO}$. The elastic option IEL would normally be zero, except for solid moderators. Currently, only the three crysialline materials graphite, beryllium, and beryllium oxide are supported for coherent elastic scattering. Options for $\mathrm{UO}_{2}$ and $\mathrm{UC}$ need to be added. If IEL=0 and TWT=0 ., an incoherent elastic section is automatically added to the ENDF tape. This normally occurs for hydrogenous solids like polyethylene, $\mathrm{ZrH}$, or frozen methane. The NSCOLD option is set to zero, exce when a liquid hydrogen or deuterium calculation is desired.

The "secondary scatterer" card would be just " $0 /$ " for simple materials like graphite or beryllium. The behavior of LEAPR for molecular moderators is determined by the value of $B 7$. The choice $B 7=0$. is for mixed moderators like $\mathrm{BeO}$ and benzine. In these cases, the entire $S(\alpha, \beta)$ for the molecule is given in $M F=7$, $M T=4$, and it is intended to be used with the neutron file for the primary scatterer. The secondary scatterer's cross section, atomic weight ratio, and effective temperature are only used for extending $S(\alpha, \beta)$ with the SCT approximation (see THERMR). When $B 7>0$., only the scattering law for the primary scatterer is given. The effects of the secondary scatterer are to be included later by using an analytic law. For example, in the Water evaluation, the $S(\alpha, \beta)$ is for $\mathrm{H}$ in $\mathrm{H}_{2} \mathrm{O}$; the oxygen is included later by using a free-gas cross section using the given SPS and AWS.

Cards 7-9 are used to define the $\alpha$ and $\beta$ grids for the LEAPR run. These grids are currently limited to 200 elements each. In the ENDF thermal format, the values for $S(\alpha, \beta)$ for the higher temperatures are given on the same $\alpha$ and $\beta$ grids as for the base temperature. But since $\alpha$ and $\beta$ are inversely proportional to $T$, only the smaller $\alpha$ and $\beta$ values would be seen at higher temperatures. The results for the higher values would normally be zero. This is a waste of space in the fields on the ENDF evaluation. Using LAT $=1$ will spread the scatter- 
ing law values for the higher temperatures out, thereby giving : more accurate representation.

The range of $\beta$ helps determine the high-energy limit for the evaluation. For example, if $T$ is $296 \mathrm{~K}$, a value of $\beta_{\max }$ of 160 will allow downscatter events of 4 $\mathrm{eV}$ to be represented without recourse to the SCT approximation. This implies that pretty good results would be obtained for incident neitrons with energies of $4 \mathrm{eV}$. If incident energies are limited to $\beta_{\mathrm{max}} k T$, the range of $\alpha$. values that can be obtained using Eq.(2) is limited to $\alpha_{\max }=4 \beta_{\max } / A$. The specific points in the $\alpha$ and $\beta$ grids are hard to choose. Too many points make the evaluation expensive to use; too few lead to inaccurate interpolated results. The low $\beta$ grid should probably have about the same detail as the input $\rho(\beta)$ in order to reflect all the structure in the frequency distribution. Because of the smoothing effect of the convolutions, the grid can gradually get coarser as $\beta$ increases. GA traditionally used a log spacing in this higher region. If translational modes are wo be included, a finer $\beta$ grid for small $\beta$ may be required to get good results at small $\alpha$. If discrete oscillators are included, additional $\beta$ values might be needed near the values $\pm n \beta_{i}$ and their various sums and differences, especially for $n=1$. After running LEAPR, the user should examine the results vs $\beta$ printed out on the listing and the results $v s \alpha$ printed out on the ENDF file to see whether the features of $S(\alpha, \beta)$ are being represented well enough. If the normalization and sum rule checks are not being satisfied well, this may be an indication that the grids are too coarse.

If a secondary scatterer with $B 7=0$. is seen, it is necessary to read two entire temperature loops, one for the principal scatterer, and one for the secondary scatterer. See the $\mathrm{BeO}$ example below for how this is done.

LEAPR aliows you to change the input frequency distribution $\rho(\epsilon)$ for every temperature, but this has not been done historically. For all the examples included in this report, Cards 11-18 are given for the first temperature only. Subsequently, only Card 10 is given to enter the desired temperalure value.

The input distribution is given as a function of energy (eV) on a uniform grid. It can be in arbitrary units; it will always be normalized to the value TBETA. If a translational term is desired in addition, set TWT to a number greater than zero. The translational term can be either a free-gas law $(c=0$.) or a diffusive law $(c>0$.). 
Card 14 is used to enter the number of discrete oscillators desired. Their energies and weights are given on the folloving two cards. It is important to obey the restriction

$$
w_{i}+w_{s}+\sum_{i=1}^{P} w_{i}=i .
$$

Of course, if ND=0, the sum over $i$ in this equation is omitted.

Card 17 is only given for the liquid hydrogen or liquid deuterium cases. It controls the entry of the pair-correlation function used to account for intermolecular interference at very low neutron energies. Card is gives the actual values for $S(\kappa)$. See the liquid hydrogen example below for an example of how this quantity is entered.

The final section of the input deck gives the new comment cards to be added to the section $\mathrm{MF}=1 / \mathrm{MT}=451$ on the ENDF file generated by LEAPR. If this section is to be a part of a standard library like ENDF/B-VI, there are standard fields that must appear. Examples of the appearance of such a formal section will be found in the following sections. Note that the comment cards are terminated by an empty card; the number of cards entered is counted by LEAPR. 


\section{BERYLLIUM METAL}

The phonon dispersion curves for metallic beryllium were fitted by Schmunk et al. ${ }^{14}$ using a model of central forces that extend to the fifth nearest neighbors. The phonon spectrum corresponding to this model was calculated by the root sampling method, and then used to compute $S(\alpha, \beta)$. It was necessary to make one small change because the $\rho(\epsilon)$ given in the GA report did not follow an $\epsilon^{2}$ law at low $\epsilon$ as required by theory. Therefore, the lowest value was changed from .00312 to .0062 . The LEAPR input file is shown below. 'The points added to the $\alpha$ and $\beta$ grid are distinguished by a different number of significant figures or a different style.

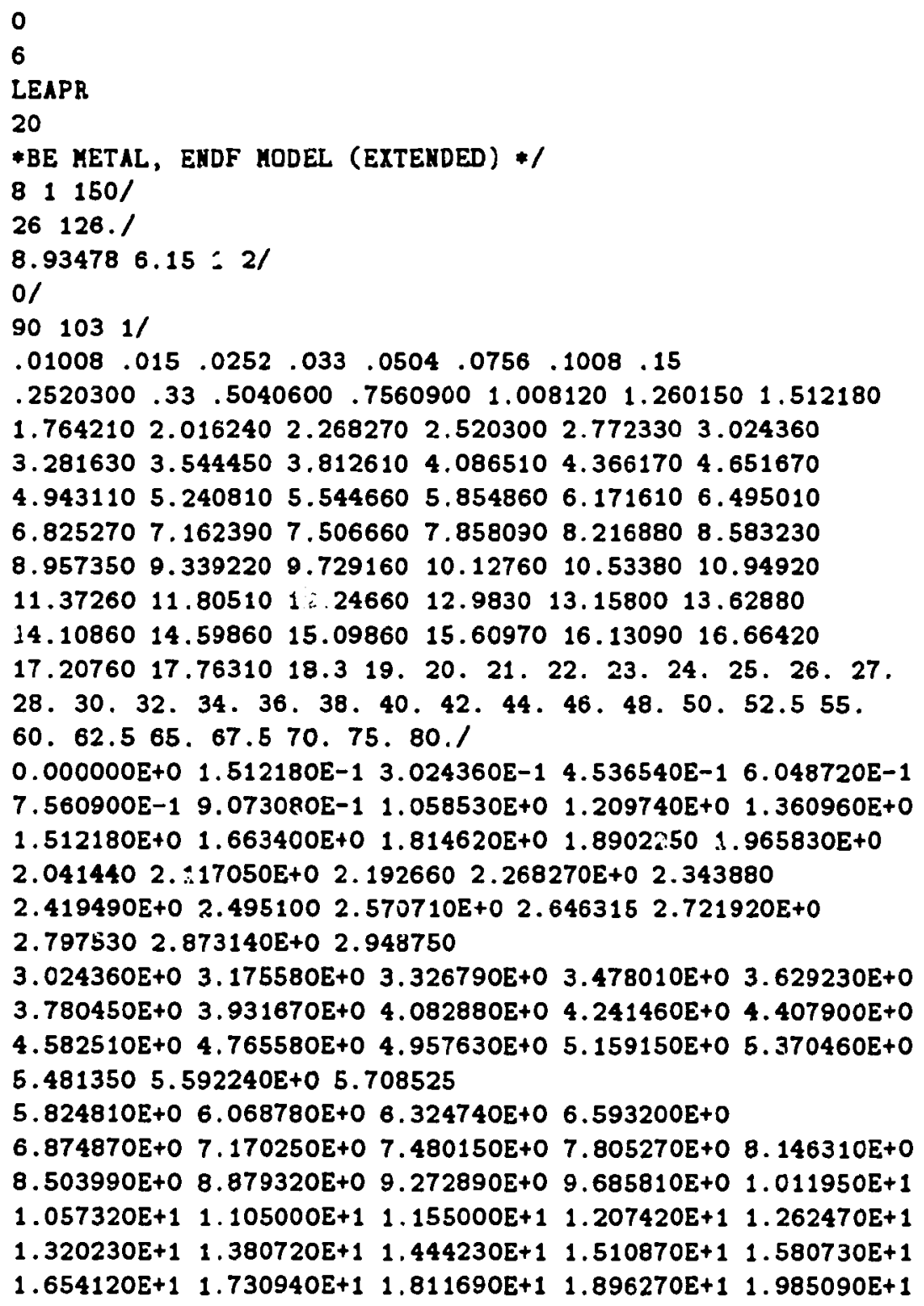


$2.078240 E+12.175920 E+12.278350 E+1 \quad 2.385810 E+12.498620 E+1$

$2.616880 E+1 \quad 2.740980 E+1 \quad 2.871120 E+1 \quad 3.007720 E+1 \quad 3.150880 E+1$

$3.301190 \mathrm{E}+1 \quad 3.458760 \mathrm{E}+1 \quad 3.624090 \mathrm{E}+1 \quad 3.797490 \mathrm{E}+1 \quad 3.979450 \mathrm{E}+1$

42. 44. 46. 48. 50. 52.5 55. 57.560 .65 .70 .75 . 80./

$296 /$

$.001536155 /$

$0 . \quad .0062 \quad .025 \quad .06 \quad .115 \quad .17 \quad .235$

$\begin{array}{lllllll}.308 & .39 & .475 & .595 & .775 & 1.052\end{array}$

$\begin{array}{lllllll}1.1482 & 1.6233 & 1.5653 & 1.7423 & 2.4289 & 2.8072\end{array}$

$3.2863 \quad 3.75774 .43975 .49246 .33157 .6421$

$0.5339 \quad 12.1016 \quad 15.0553 \quad 22.0154 \quad 26.6382 \quad 29.3387$

$32.6036 \quad 35.777 \quad 37.6536 \quad 40.3845 \quad 37.1196 \quad 32.39$

$\begin{array}{llllllll}29.8269 & 21.2831 & 18.9488 & 13.0186 & 7.33848 & 12.5075\end{array}$

$\begin{array}{lllllll}18.9031 & 23.5564 & 25.6923 & 25.5855 & 43.0544 & 17.8658\end{array}$

$\begin{array}{llllllll}6.81975 & 3.98963 & 2.99184 & 1.6843 & .65772 & 0 . /\end{array}$

0.0 .1 .0 .1

o/

$-400.1$

$-500.1$

$-600.1$

$-700.1$

$-800.1$

$-1000 . /$

$-1200 . /$

* BE METAL LARL EVAL-APR93 MaCFARLARE */

* REF. 3 DIST $-* 1$

- - - ENDF/B-6

MATERIAL $26 * 1$

* ---- THERMal heUtron scatTERIhg data */

$*-----$ ENDF-6*1

$*+1$

* tenperatures $=296,400,500,600,700,800,1000,1200 * 1$

* *1

* BISTORY */

* - - - - - * *

* teis evaluation has gemerated at tee los alamos matiogal *

* laboratory (APR 1993) USIMg the leapr code. the PEYSical */

* hodel is very similar to the one USEd at general atomic *

* in 1969 to produce tae original endf/B-III eValuations *

* (SEE REF. 1). TIGHTER gRIdS aHd EXTENDED RAHGES FOR aLPHa */

* and beta nere uSEd. a slightly more detailed calculation */

* of tae coherent imelastic scatterimg has geBerated. of *

* COURSe, tae various constaAts were updated to agReE HIT日 */

* THE ETdF/B-VI EVAluation of BE. */

* $* 1$

* taeory *1

$+----D+1$

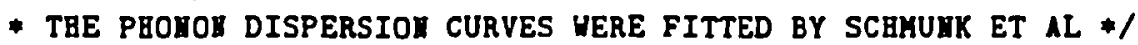

* Using a yOdel of central forces that extend to THE fifth *

* hearest heightbors (Ref 2). the phoHon spectruk correspohdié */

* to tais yOdel has calculated by taE roOt SAMPling METHOd, */

* and teen used to compute s(alpha,beta). tae coberent elastic *

* scattering cross section has computed using the knowg lattice *

* structure (hexagonal close-packed) ayo tae debye-yaller *

* integrals fron the latTice dyayics model. *

$* \quad 1$ 
* - - - - $-2-2-1$

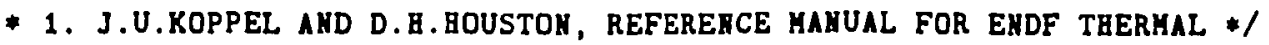

* HEUTROI SCATTERIMg DATA, GENERAL ATOKIC REPORT Ga-8774 */

* revised aHd reissued as endF-269 by tae matiogal guclear */

* data center, july 1978. */

* 2. R.E.SCHYUBK, R.A.BRUGGER, P.D.RAHDOLPB, ABD K.A.STROEG,*/

* PBYS. REV. 128,562 (1962). *I

* 3. R.E.MACFARLAHE, BEH THERMaL HEUTRO SCATTERIBg FILES FOR */

- ERDF/B-VI-RELEASE 2, LOS ALAHOS MATIOHAL LABORATORY REPORT */

* IA-12639-MS (TO BE PUBLISBED). */

$* * 1$

1

STOP

The value $\alpha_{\max }=80$ was selected to allow for incident energies as high as $4.5 \mathrm{eV}$. Actually, at high energies, the energy loss in downscatter is limited by kinematic effects to about $.36 \times E$. This corresponds to $\beta$ values below about 65 for $4.5 \mathrm{eV}$. A value of $\beta_{\max }$ of 80 will be sufficient to include this limit, and it will also allow the normalization and sum-rule checks of LEAPR to be computed more accurately at high $\alpha$. The phonon frequency spectrum given in this input file is plotted in Figure 1.

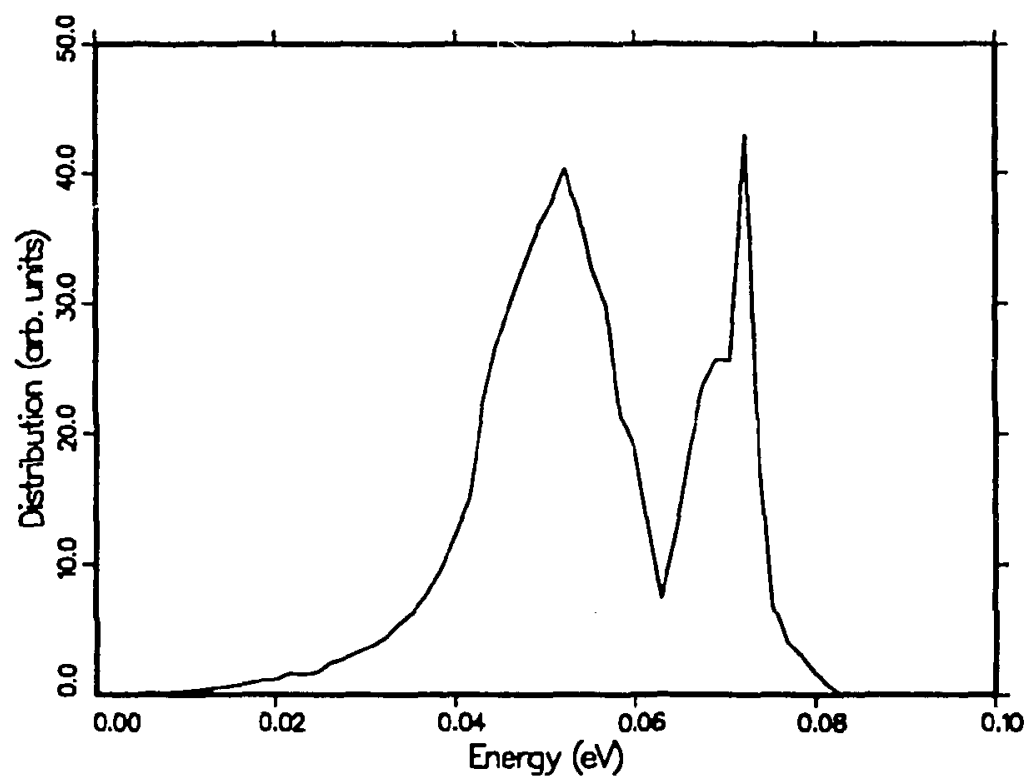

Figure 1: The phonon frequency spectrum $\rho(\epsilon)$ used for Be metal.

The resulting $S(\alpha, \beta)$ is shown in Figures 2 through 9. The first two figures show $\mathrm{S}$ as a function of $\alpha$ at various $\beta$ values featuring low $\alpha$ and high $\alpha$, respectively. The agreement between the new calculations and ENDF/B-VI.0 is 
fairly good, except that the old values only extend from $\alpha$ equals .25 to 17.76. The disagreements just below $\alpha=17.76$ are due to the use of the SCT approximation in GASKET. The lack of agreement for $\beta=0$ is caused by the error in $\rho(\beta)$ in the old evaluation. The scallops seen at low $\alpha$ are caused by the lin-log interpolation used in ENDF. They are not a problem in practice, because THERMR uses a three-point quadratic interpolation to determine $S(\alpha, \beta)$ between grid points. The quadratic interpolation also helps to give better values for the concave-downward regions at the peak of the scattering law. The effect of the very different lower limits on $\alpha$ is somewhat alleviated in THERMR, which extrapolates to small $\alpha$ using the $\log$-log method.

The next two figures show $S(\alpha, \beta)$ at $1200 \mathrm{~K}$. The results are a little smoother at this higher temperature.

Figures 6 through 9 show $\mathcal{S}(\alpha,-\beta)$, that is, script-S for downscatter, at both 296 and $1200 \mathrm{~K}$. They reveal that the GASKET calculations tend to break down for small values of the scattering law $S$. The shapes of the curves tend to become simpler at high $\alpha$, but the presence of the curves for large $\alpha$ is necessary to get accurate cross section values for energy transfers of more than $1 \mathrm{eV}$. Plotting script-S versus $\alpha$ gives a better idea of the importance of the large $\alpha$ values than that given by Figures 2 and 3. Using a three-point interpolation scheme in THERMR is important to get good integrals over the sharply concave-downward curves at high $\alpha$ and $\beta$.

Figures 10 and 11 give two views of the integrated inelastic cross section for Be. The agreement between the new evaluation and ENDF/B-VI.0 is excellent. Figure 12 shows the coherent elastic scattering cross section for Be. The differences between .08 and $.4 \mathrm{eV}$ at $296 \mathrm{~K}$ are due to problems in translating the ENDF/B-III evaluation to ENDF-6 format.

Figures 13 through 16 show some of the secondary neutron spectra for Be. The differences between the new evaluation and ENDF/B-VI.0 are more visible here, but they are still not important. The notch at $E^{\prime}=E$ in Figure 14 is due to the non-quadratic behavior of $\rho(\epsilon)$ near $\epsilon=0$ in the old evaluation.

A table of effective temperatures and Debye-Waller integrals is given at the end of this section.

It is clear that this new evaluation is only a very slight improvement over the old one, except for coherent elastic scattering. This result gives increased confidence in existing calculations, and it provides some validation for LEAPR. 


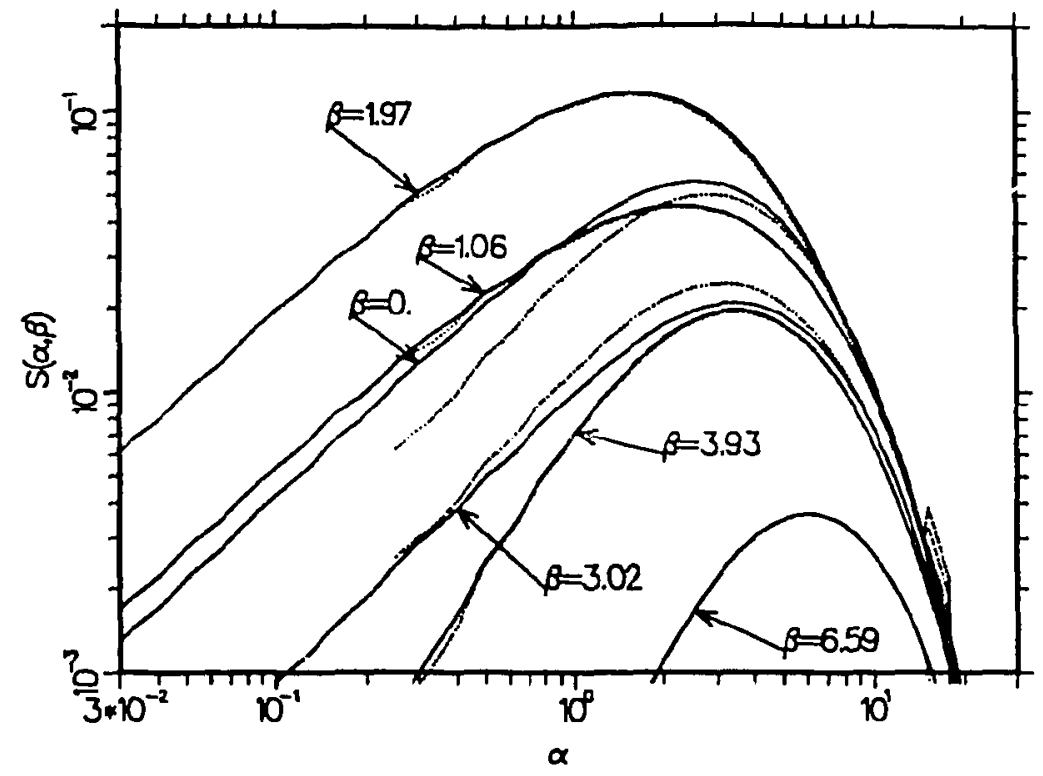

Figure 2: $S(\alpha, \beta)$ vs $\alpha$ for several $\beta$ values at a temperature of $296 \mathrm{~K}$ for Be emphasizing the low- $\alpha$ side of the function. The solid lines are the results of this calculation, and the dashed lines are for ENDF/B-VI.0.

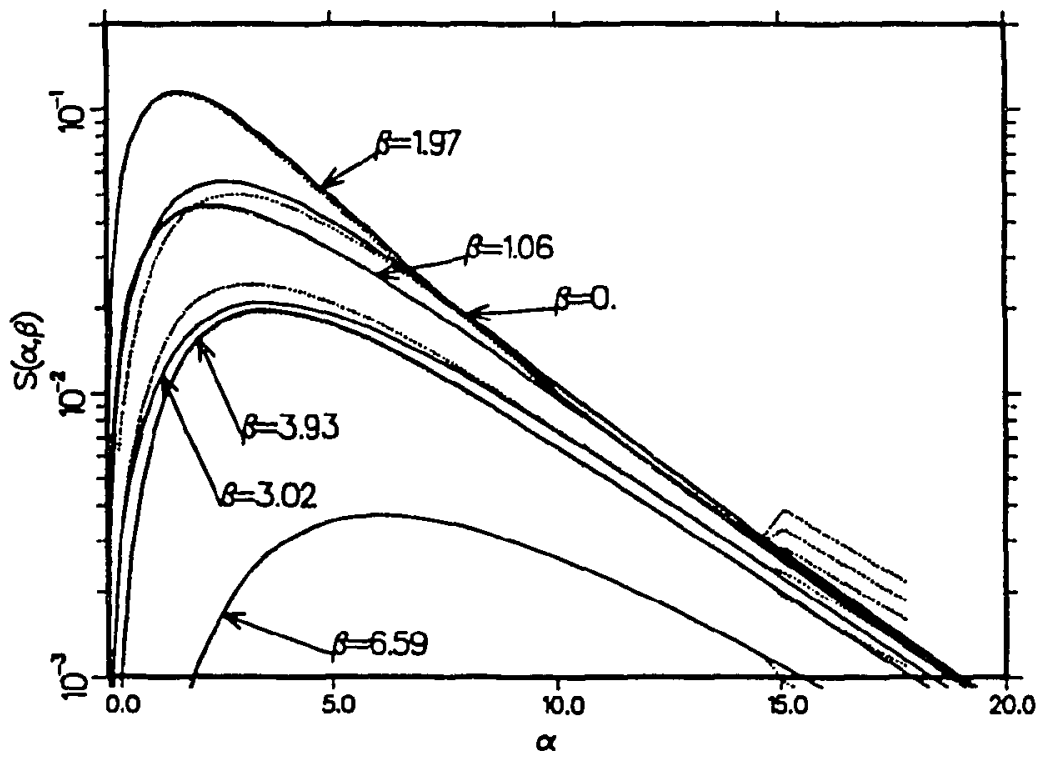

Figure 3: $S(\alpha, \beta)$ vs $\alpha$ for several $\beta$ values at a temperature of $296 \mathrm{~K}$ for Be emphasizing the high- $\alpha$ side of the function. The solid lines are the results of this calculation, and the dashed lines are for ENDF/B-VI.0. 


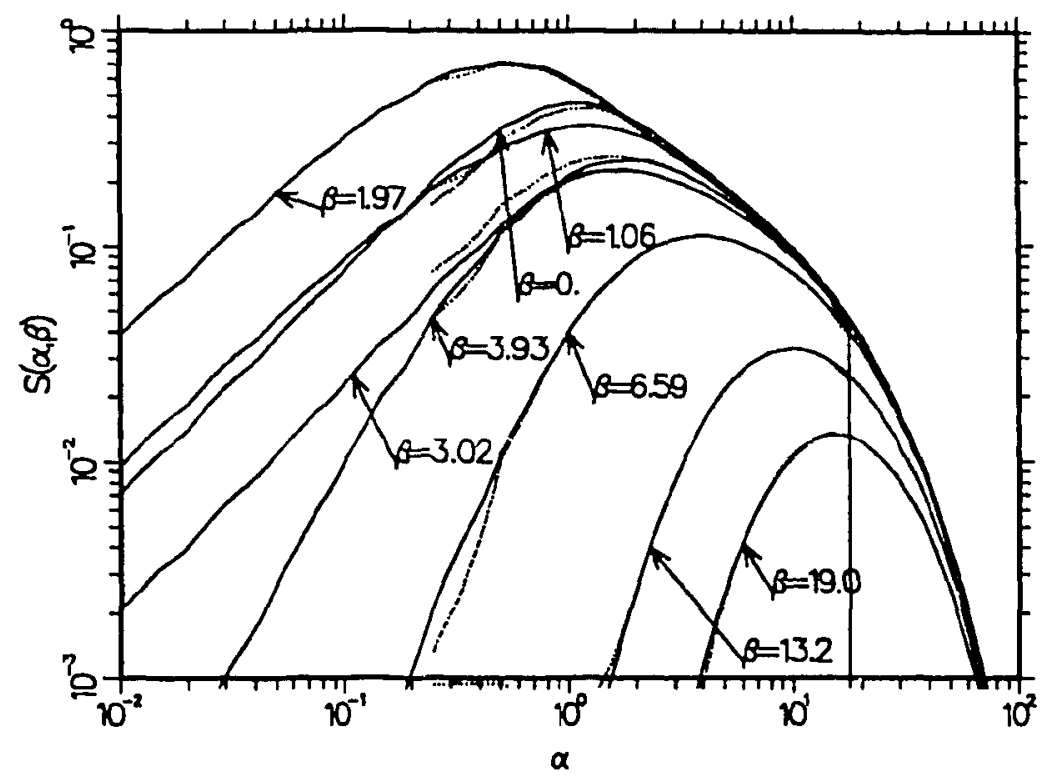

Figure 4: $S(\alpha, \beta)$ vs $\alpha$ for several $\beta$ values at a temperature of 1200 $\mathrm{K}$ for Be. The solid lines are the results of this calculation, and the dashed lines are for ENDF/B-VI.0

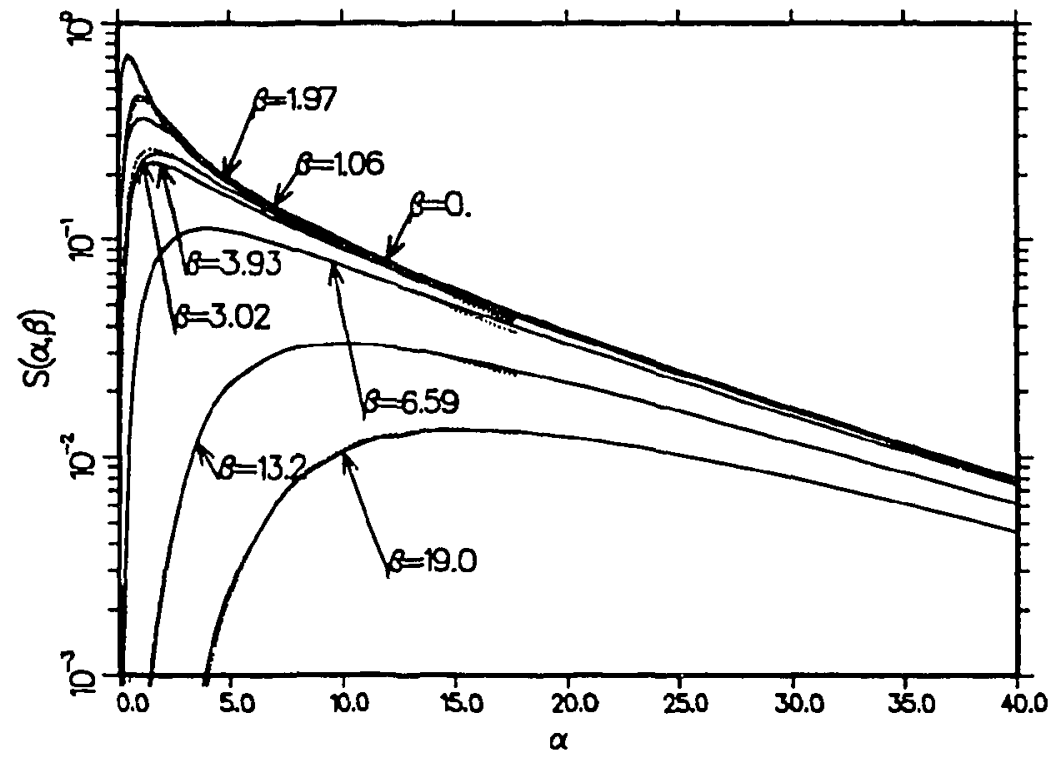

Figure 5: $S(\alpha, \beta)$ vs $\alpha$ for several $\beta$ values of at a temperature of 1200 $\mathrm{K}$ for $\mathrm{Be}$. The solid lines are the results of this calculation, and the dashed lines are for ENDF/B-VI.0. The effects of the new evaluation are similar to those seen at $296 \mathrm{~K}$. 


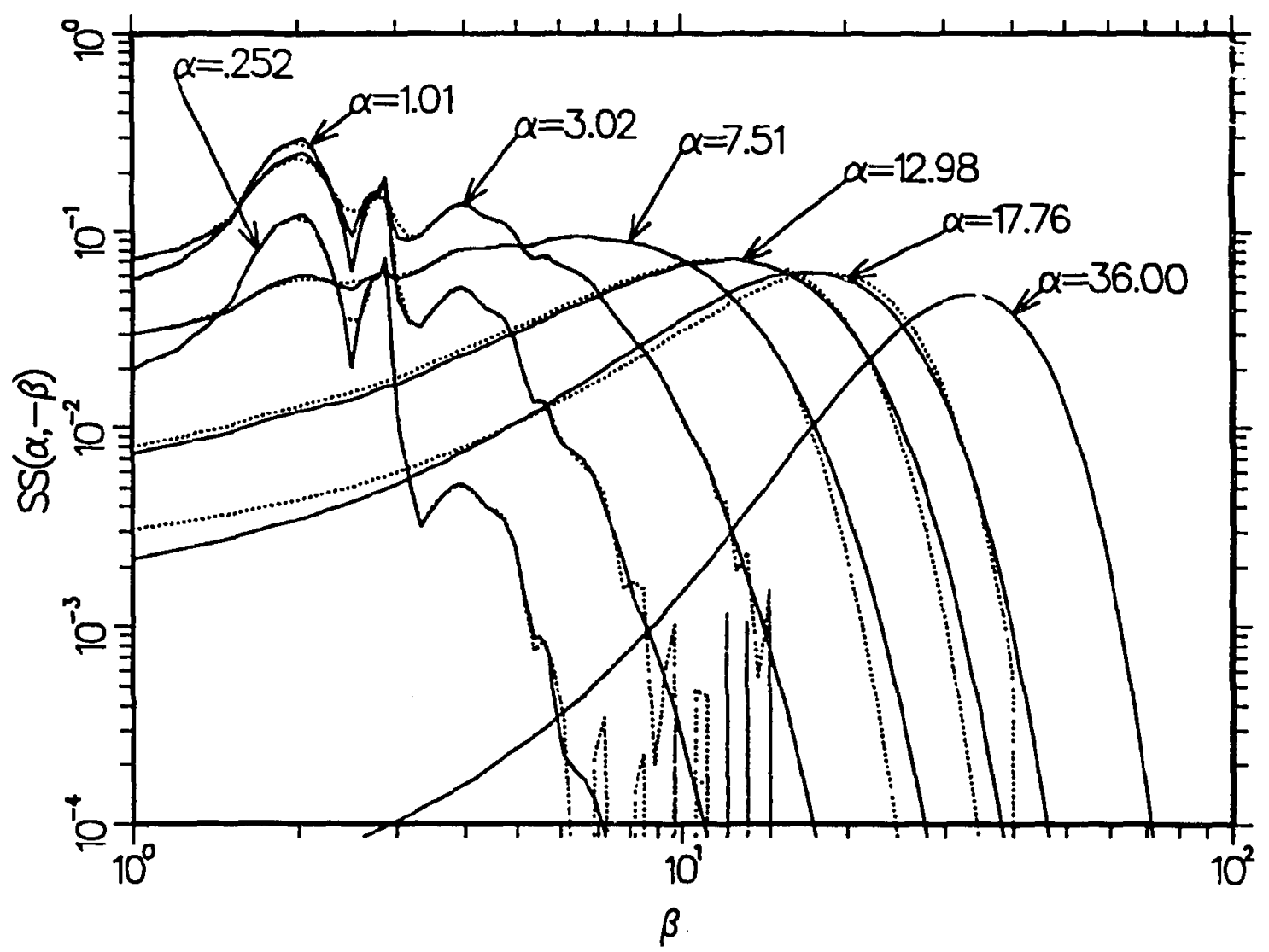

Figure 6: $\mathcal{S}(\alpha,-\beta)$ vs $\beta$ for several $\alpha$ values at a temperature of 296 $\mathrm{K}$ for $\mathrm{Be}$. The solid lines are the results of this calculation, and the dashed lines are for ENDF/B-VI.0. The finer $\beta$ grid used in the new evaluation does a better job of following the peaks and valleys resulting from features in $\rho(\beta)$. Also note that the new evaluation does a better job for small $\mathcal{S}$. SS on the ordinate stands for script $\mathrm{S}$. 


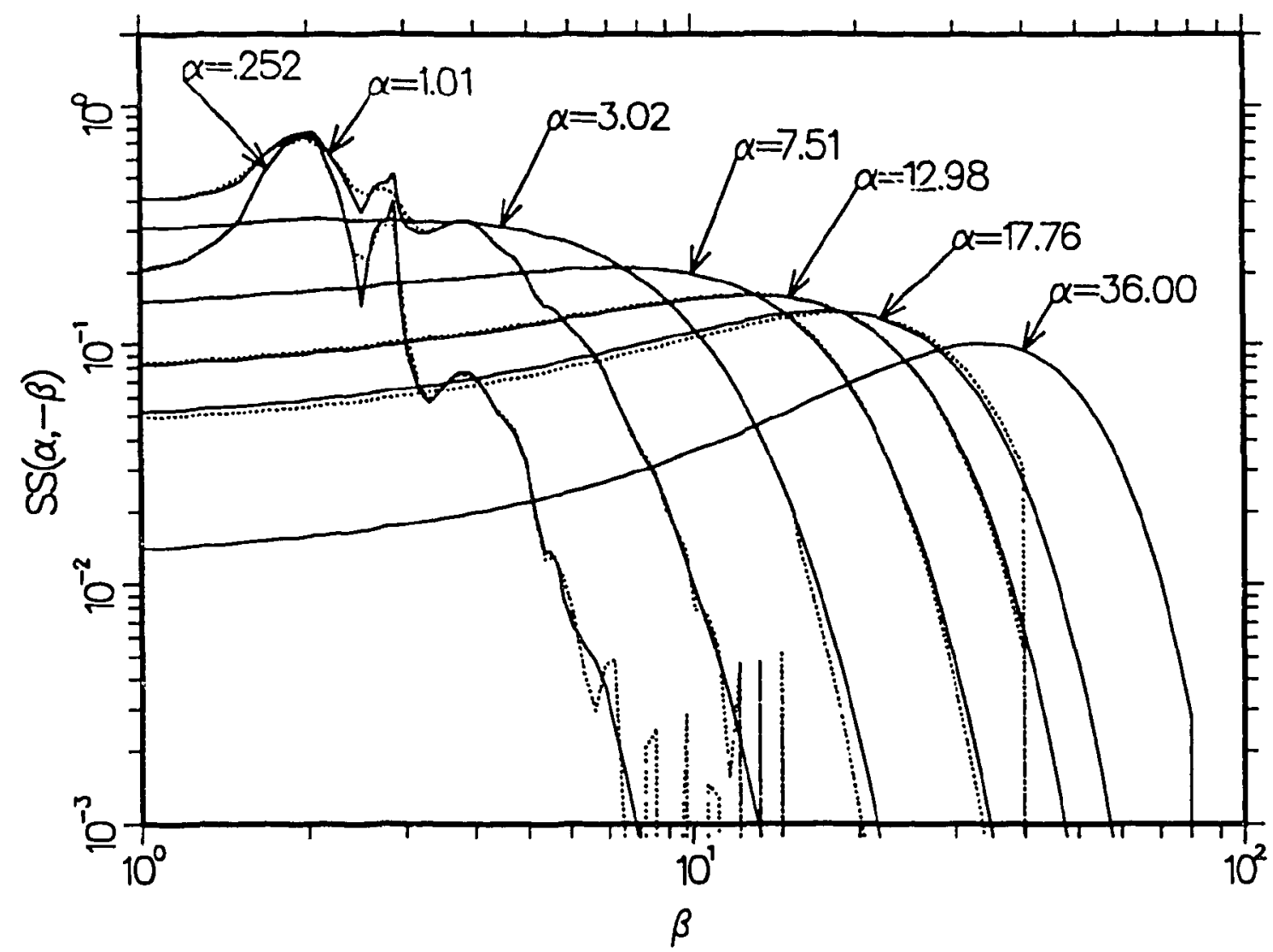

Figure 7: $\mathcal{S}(\alpha,-\beta)$ vs $\beta$ for several $\alpha$ values at a temperature of $1200 \mathrm{~K}$ for $\mathrm{Be}$. The solid lines are the results of this calculation, and the dashed lines are for ENDF/B-VI.0. The comparisons show the same effects seen at $296 \mathrm{~K}$, except the peaks and valleys are somewhat smoothed out at this high temperature. 


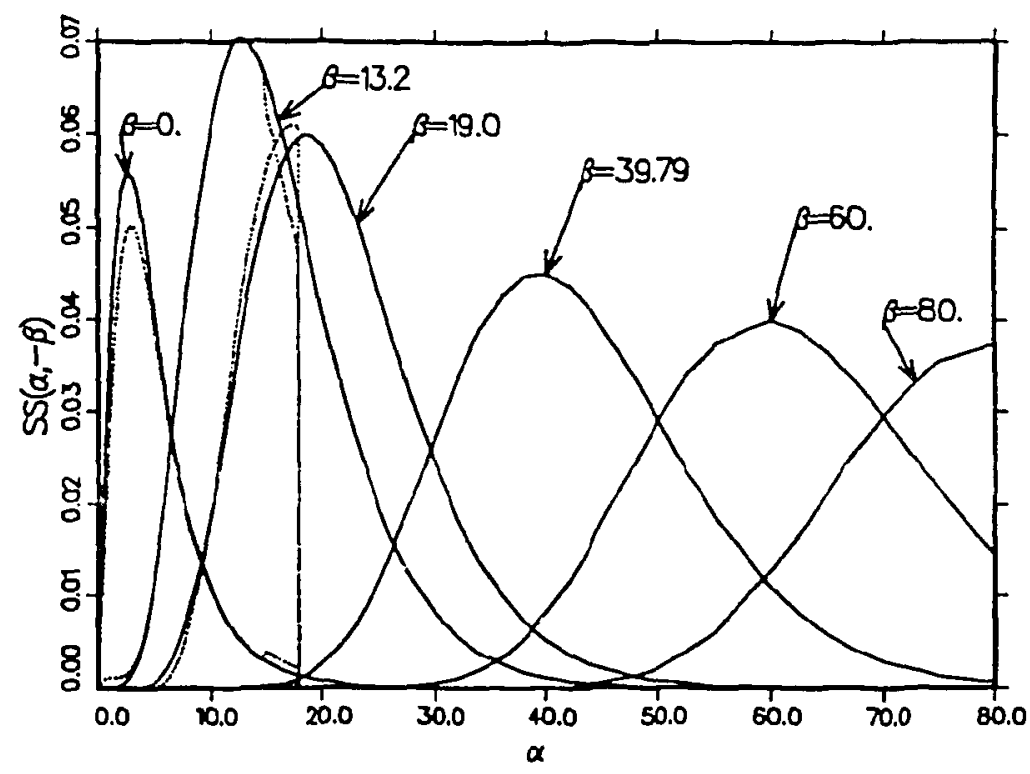

Figure 8: $\mathcal{S}(\alpha,-\beta)$ vs $\alpha$ for several $\beta$ values at a temperature of 296 $\mathrm{K}$ for $\mathrm{Be}$. The solid lines are the results of this calculation, and the dashed lines are for ENDF/B-VI.0.

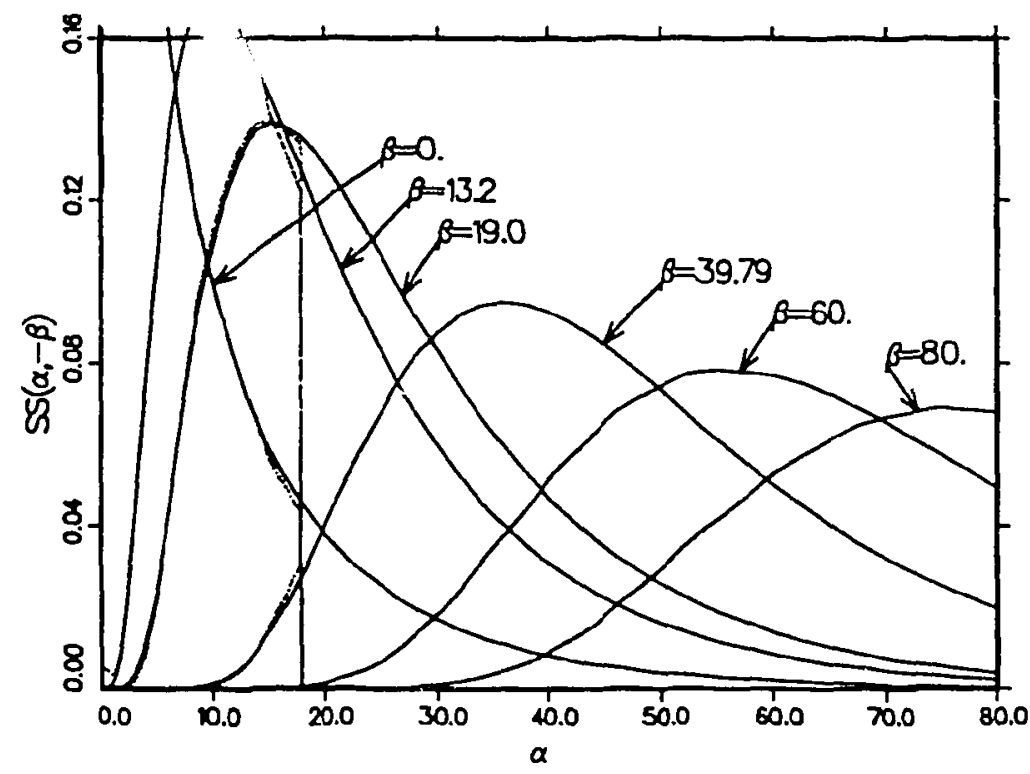

Figure 9: $\mathcal{S}(\alpha,-\beta)$ vs $\alpha$ for several $\beta$ values at a temperature of 1200 $\mathrm{K}$ for $\mathrm{Be}$. The solid lines are the results of this calculation, and the dashed lines are for ENDF/B-VI.0. 


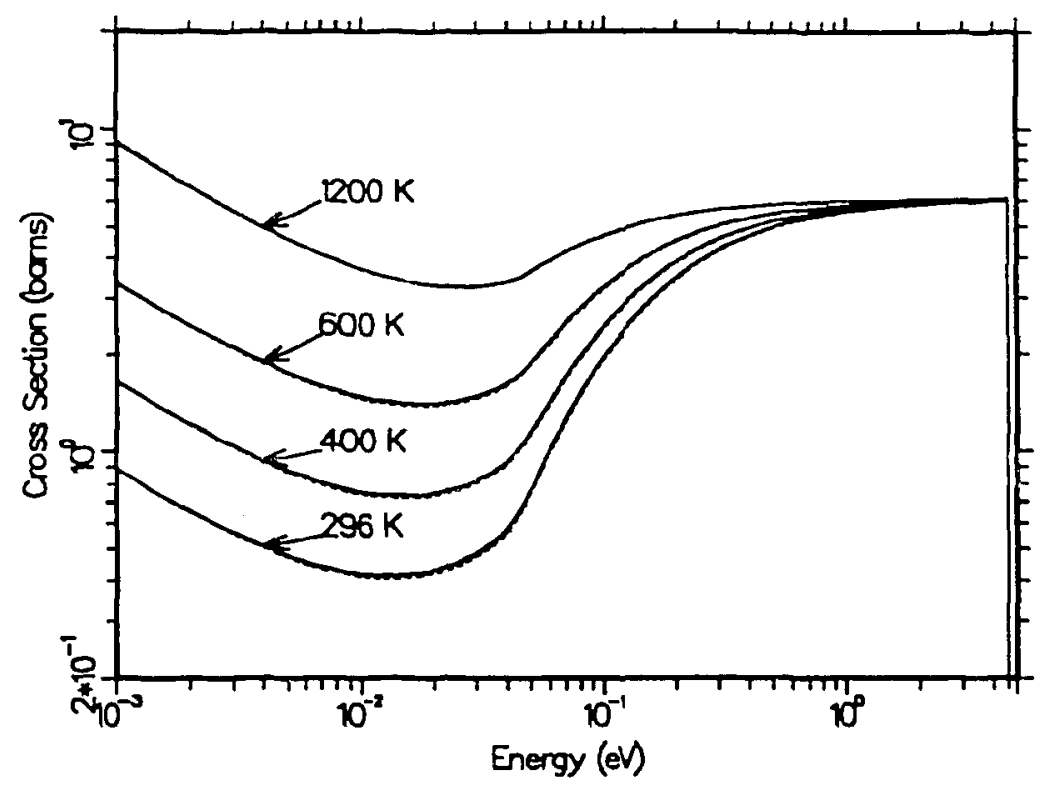

Figure 10: The inelastic cross section for $\mathrm{Be}$ at four temperatures emphasizing the low-energy range. The dashed curves are for the ENDF/B-VI.0 evaluations, and there is very little difference seen.

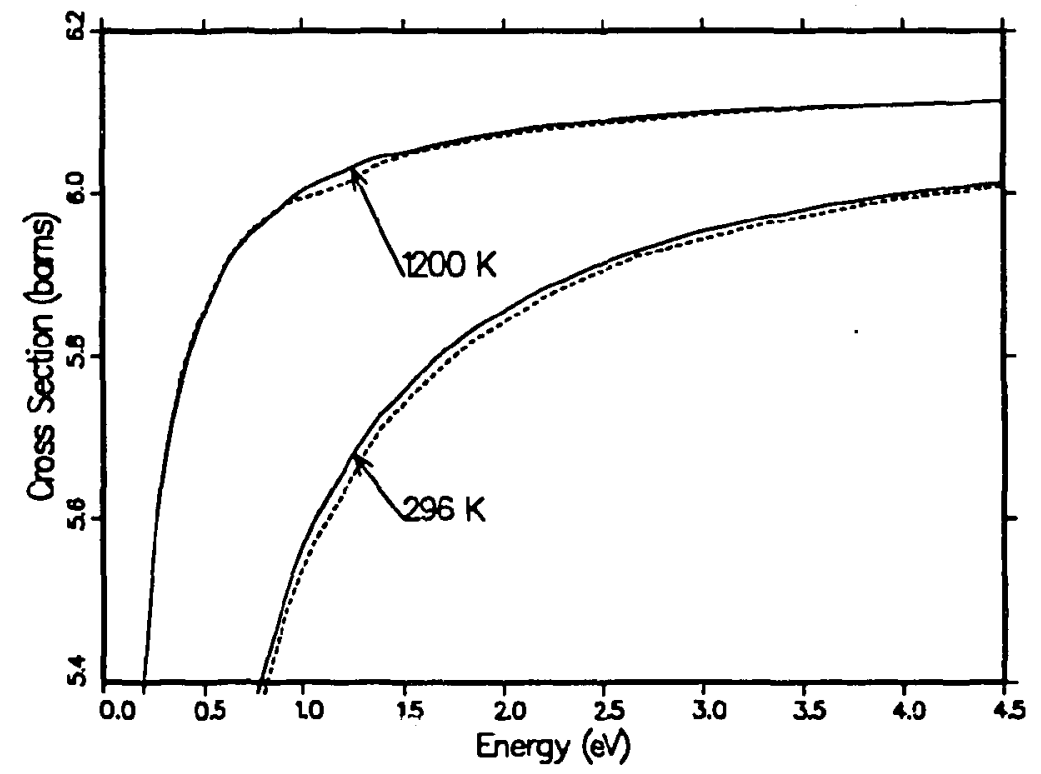

Figure 11: The inelastic cross section for Be at temperatures of $296 \mathrm{~K}$ and $1200 \mathrm{~K}$ emphasizing the high-energy range. The dashed curves are for the ENDF/B-VI.0 evaluations. Once again, little difference is seen. 


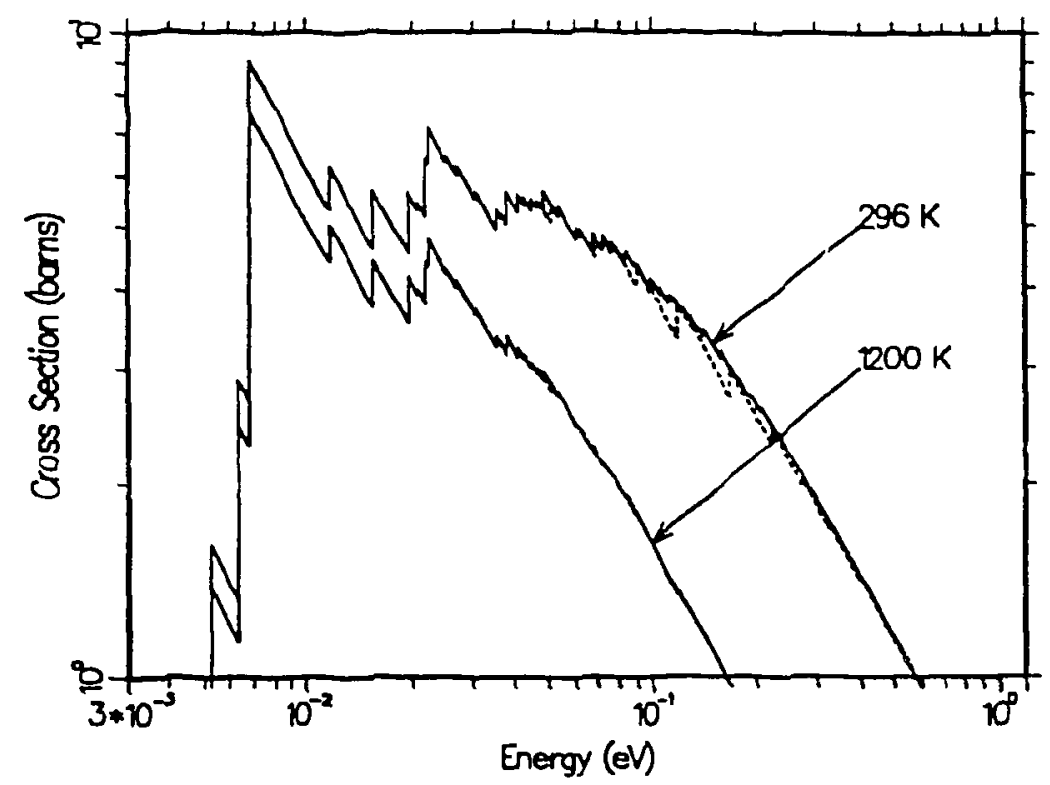

Figure 12: The coherent elastic cross section for Be at temperatures of $206 \mathrm{~K}$ and $1200 \mathrm{~K}$ showing the Bragg peaks. The dashed curves are for the ENDF/B-VI.0 evaluations. Note that the new evaluation fixes the problem in the old evaluation that is visible at $296 \mathrm{~K}$ for high energies.

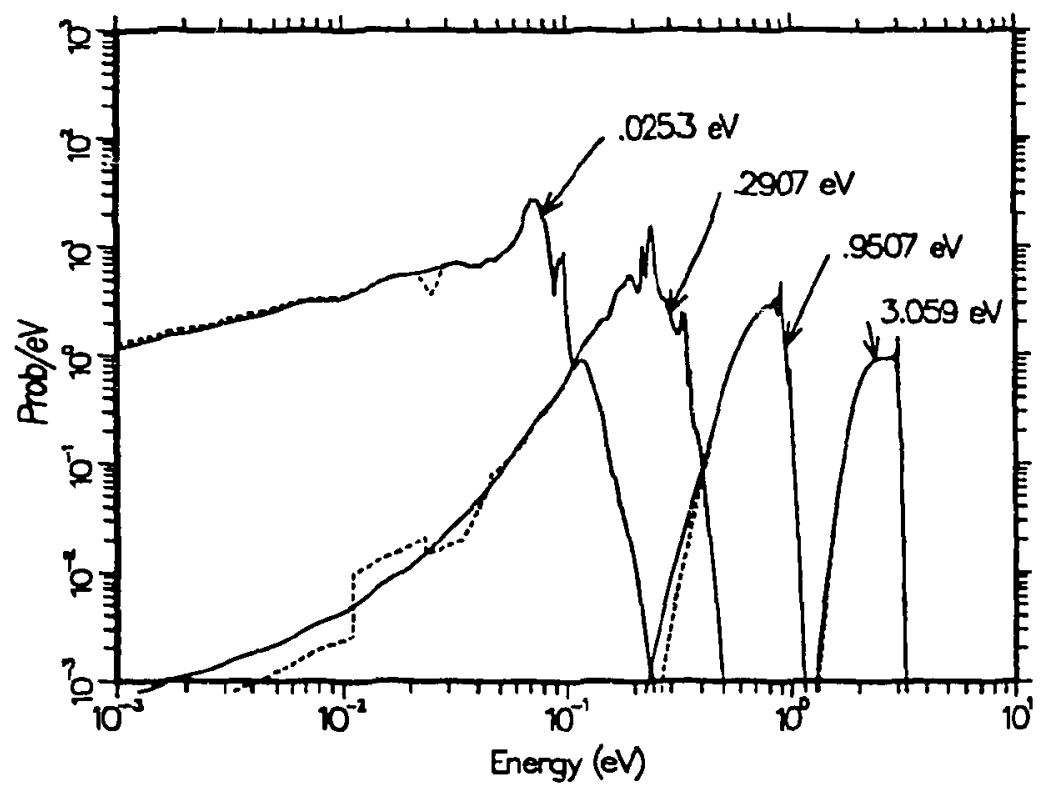

Cigure 13: Secondary neutron spectra for Be for several incident energies at a temperature of $296 \mathrm{~K}$. The dashed curves are for the ENDF/B-VI.0 evaluation. 


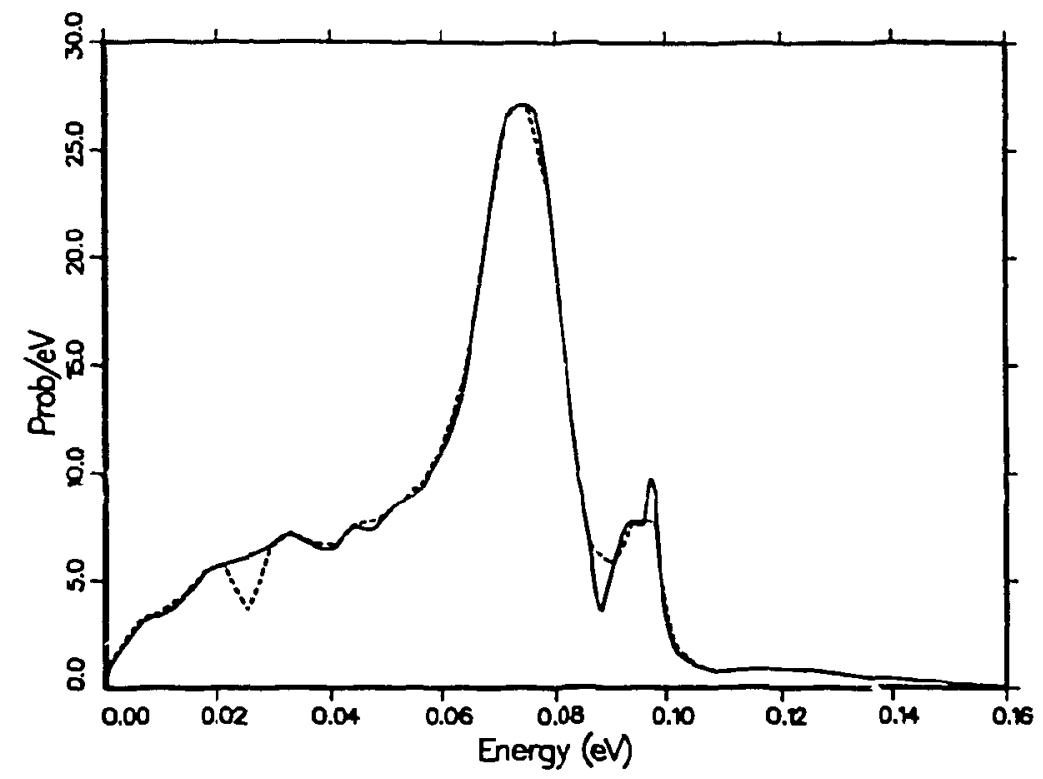

Figure 14: Expanded view of the secondary neutron spectrum at $.0253 \mathrm{eV}$ for $\mathrm{Be}$ at a temperature of $296 \mathrm{~K}$. The dashed curve is the ENDF/B.VI.0 evaiuation. The notch at $.025 \mathrm{eV}$ is due to a problem in the original frequency distribution.

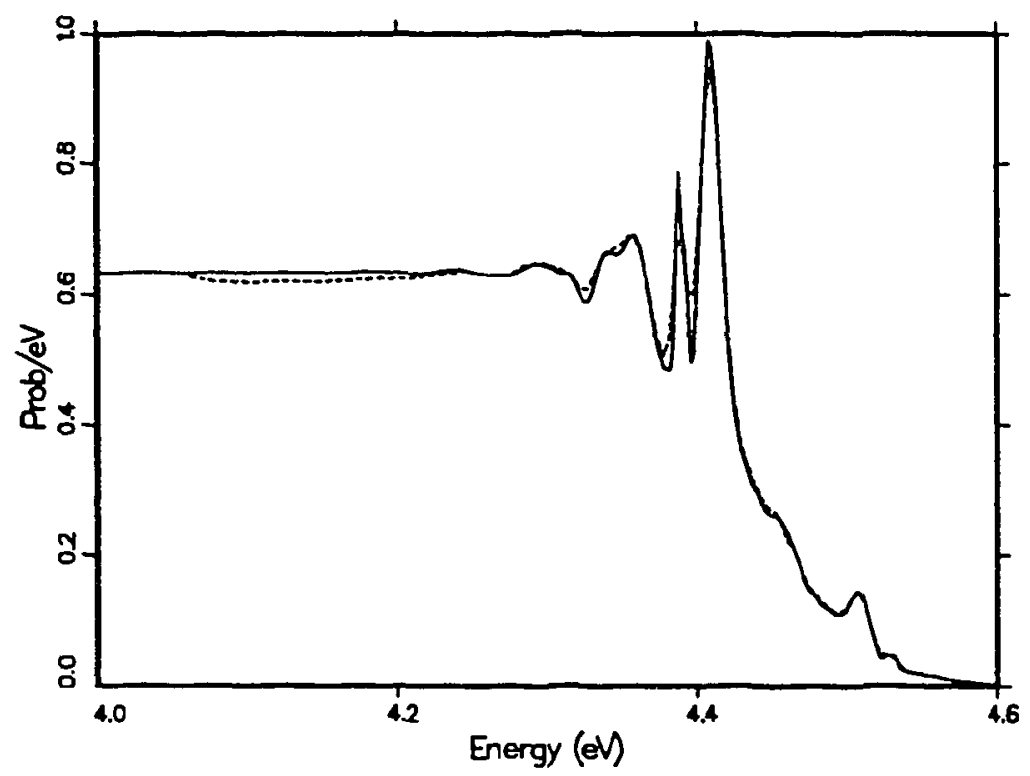

Figure 15: Expanded view of the secondary neutron spectrum at $4.46 \mathrm{eV}$ for $\mathrm{Be}$ at a temperature of $296 \mathrm{~K}$. The dashed curve is the ENDF/B-VI.0 evaluation. Note that the peaks and valleys are represented better in the new evaluation. 


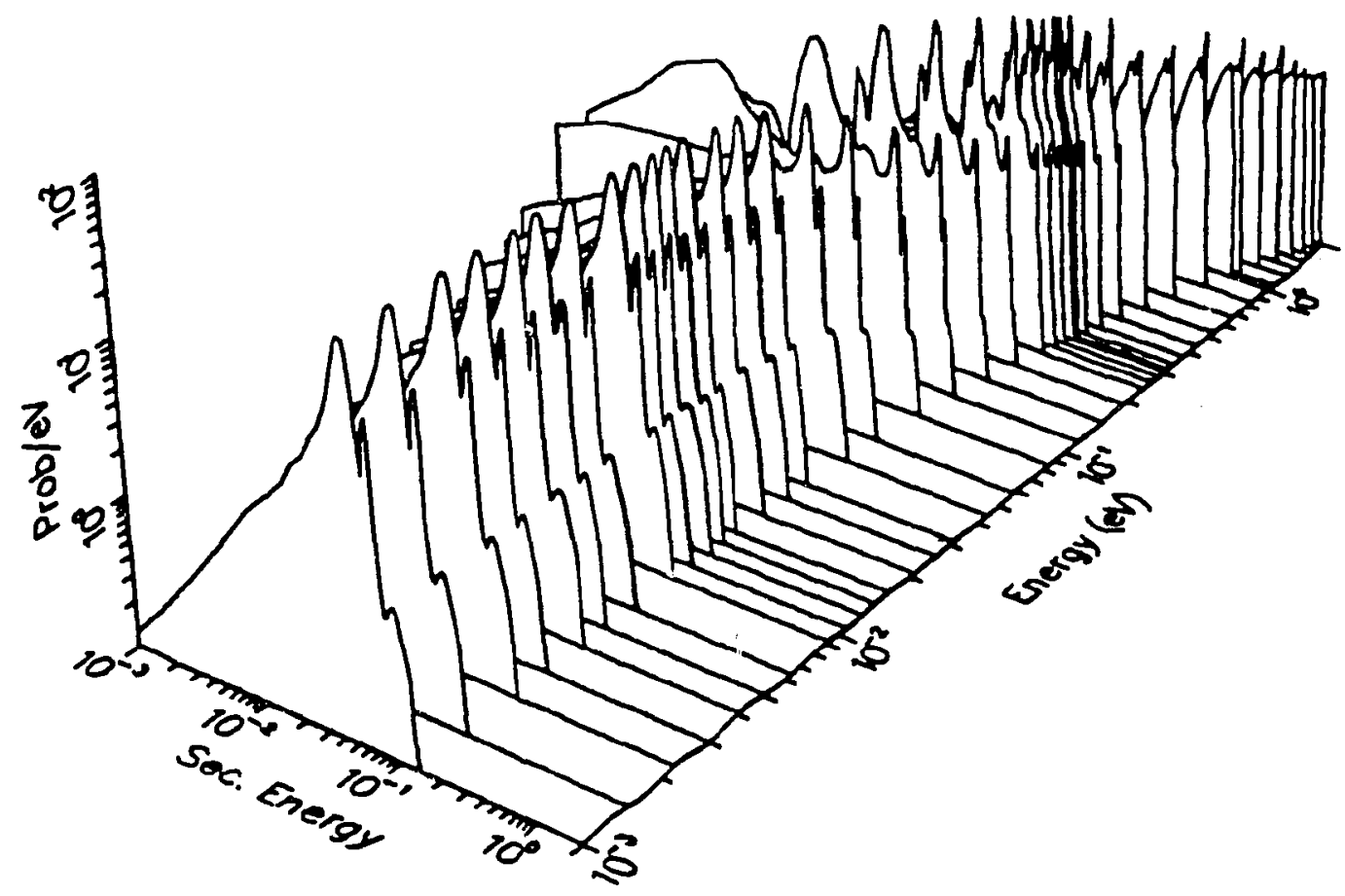

Figure 16: Perspective view of the secoudary neutron spectra from thermal inelastic scattering from Be at $296 \mathrm{~K}$ for energies up to $2 \mathrm{eV}$. 
Table 2: Table of Debye-Waller Integrals and Effective Temperatures for Be

\begin{tabular}{ccc}
\hline $\begin{array}{c}\text { Temperatuze } \\
(\operatorname{deg} K)\end{array}$ & $\begin{array}{c}\text { Debye-Waller Int. } \\
\left(\mathrm{eV}^{-1}\right)\end{array}$ & $\begin{array}{c}\text { Effective Temp. } \\
(\operatorname{deg} \mathrm{K})\end{array}$ \\
\hline 296 & 28.69 & 405.9 \\
400 & 35.21 & 484.4 \\
500 & 41.98 & 568.7 \\
600 & 49.02 & 657.8 \\
700 & 56.22 & 749.8 \\
800 & 63.53 & 843.8 \\
1000 & 78.35 & 1035. \\
1200 & 93.31 & 1229. \\
\hline
\end{tabular}




\section{BERYLLIUM OXIDE}

For this material, it is necessary to prepare a mixed $S(\alpha, \beta)$.

Once again, the basic physics was left unchanged from the GA evaluation of 1969. ${ }^{1}$ Beryllium oxide consists of two interpenetrating hcp structures with four atoms per unit cell. The lattice dynamics were developed ${ }^{15}$ on the basis of a shell model, whose parameters were chosen in such a way as to give the best agreement to the elastic constants data and to the measured Raman frequencies. Only the negative ions (oxygen) were assumea to be polarizable. The effective charge on the ions was taken to be 1.1 electron units, as derived from the Szigeti relation. The negative charge on the shell of each oxygen atom was taken to be equal to 1.2 electron units. The isotropic elastic force constant connecting the shell and core of the negative ions was taken to be equal to $3 \times 10^{5}$ dyne/cryHfithe long-renge forces were computed using the Ewald method. Short-range repulsive forces were assumed to act among first and second neighbors. These interactions take place between the positive ions and the shells of the negative ions. Introduction of the second-neighbor interaction was found to be necessary to fit the preliminary dispersion relations measured by neutron scattering. ${ }^{16}$ This model was then used to compute frequency spectra weighted by the squares of the amplitude vectors for $\mathrm{Be}$ in $\mathrm{BeO}$ and for $\mathrm{O}$ in $\mathrm{BeO}$ separately.

Note that the following input deck contains both "Be in $\mathrm{BeO}^{\text {" and }}$ " $\mathrm{O}$ in $\mathrm{BeO}^{n}$. The mixing option is selected by the " $0 .{ }^{n}$ in the second field of the 9th input card. The points added to the $\alpha$ and $\beta$ grids are fairly obvious.

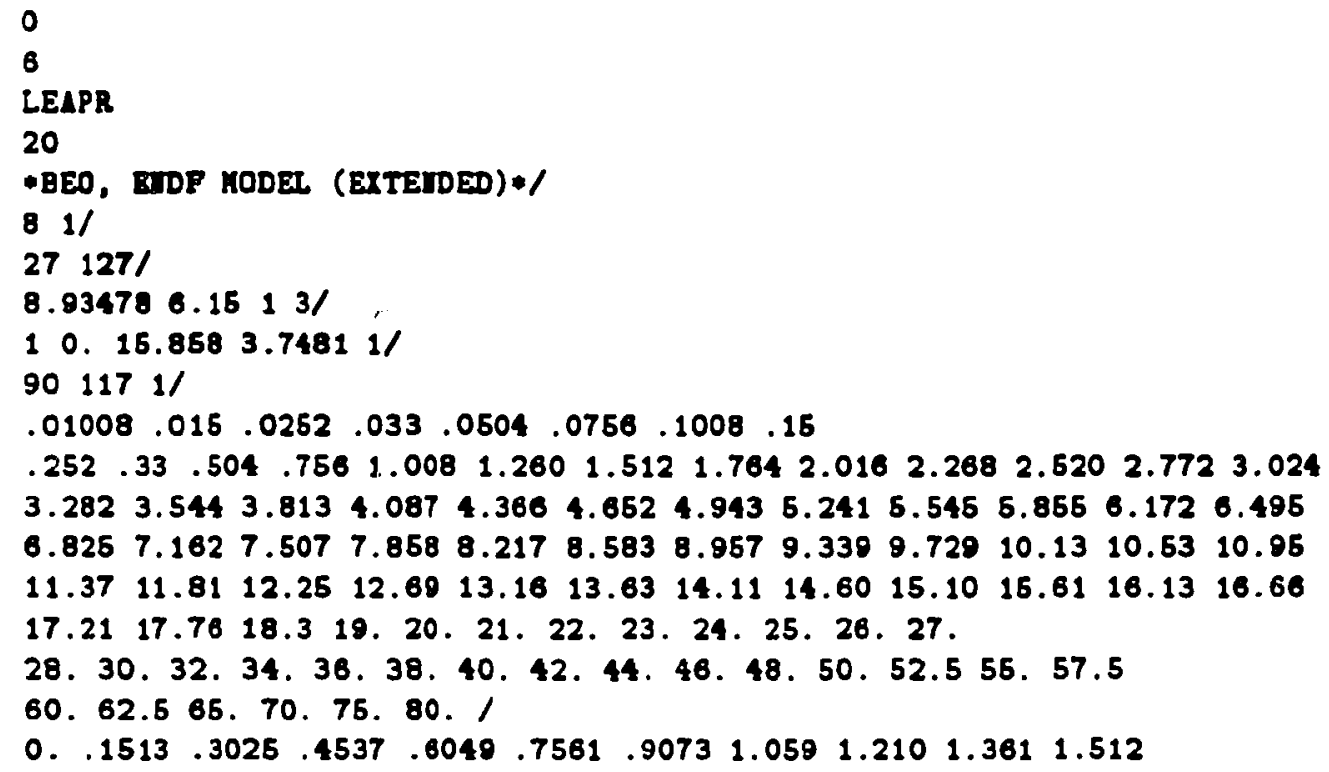




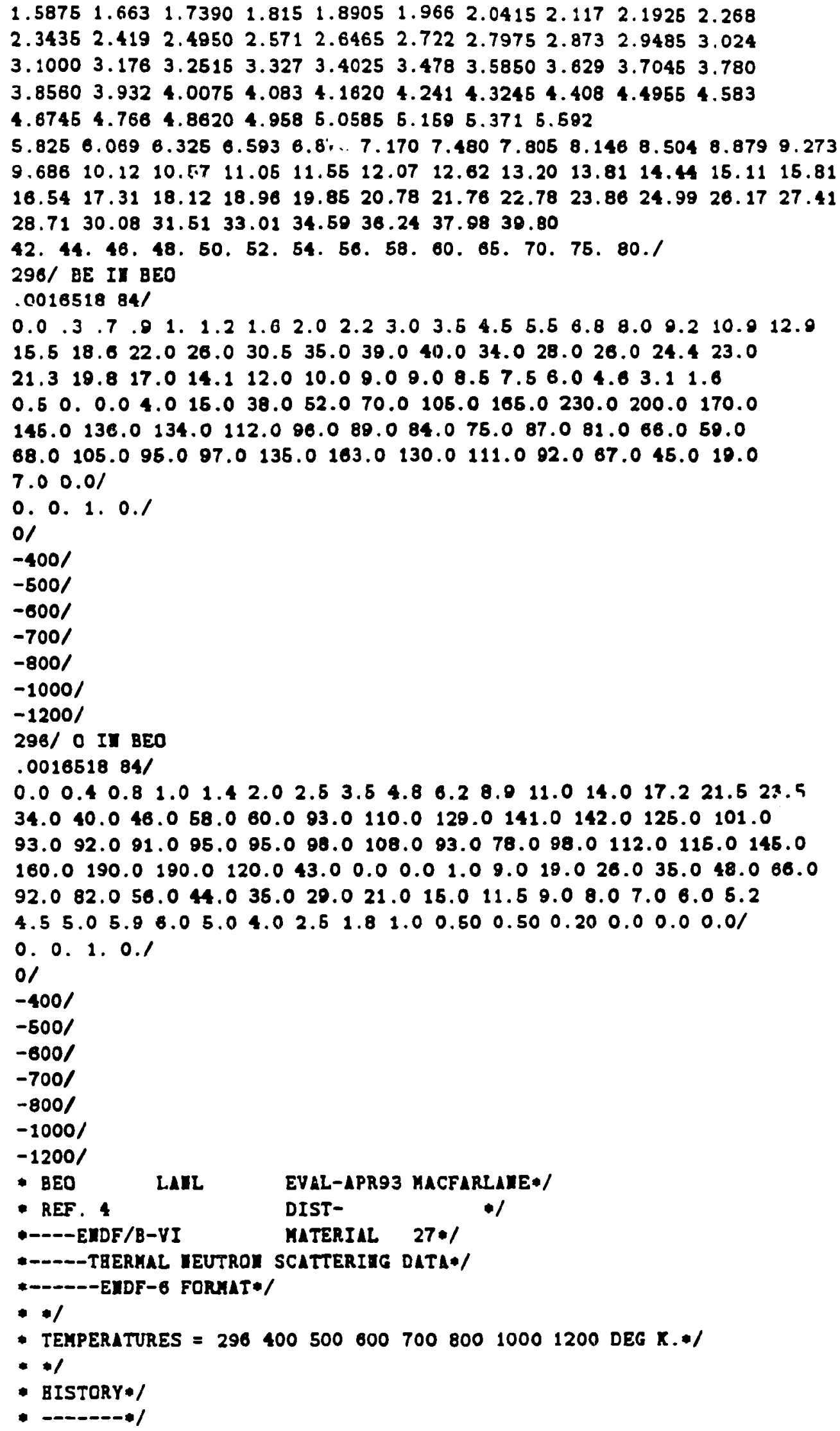


- tais eValuation has gemerated at tge los alamos matiomal *

- LABORATORY (APR 1993) USIIG TEE LEAPR CODE. TEE PBYSICAL */

- hodel is very similar to tee ote used at gemeral atomics */

- II 1968 TO PRODUCE THE ORIGITAL EIDF/B-III EVALUATION */

* (SEE REF. 1.). TIGETER GRIDS aID EITEIDED RAMGES FOR LLPHA *

- amd beta vere used. a sligatry more detailed calculatiod */

- of the cobereut imelastic scatterimg has gemerated. of *

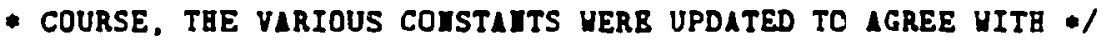

* the emdF/B-vi evaluations of be atd oxygen. *

$* 1$

- TEEORY*/

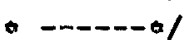

- BERYLIIUN OIIDE COISISTS OF TUO IUTERPEIETRATIJG BETAGOTAL*/

- CLOSE-PACKED STRUCTURES uITE FOUR ATOMS PER UTIT CELL. THE*/

- LATTICB DYIAMICS (REF.2) IS DESCRIBED USIJg $\triangle$ SBELL MODEL*/

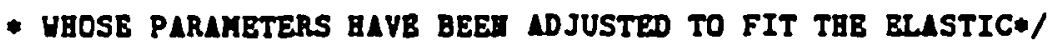

- COISTAITS, teB MEASURED RAMAI FREqUEJCIES, AID PRELIMITART*/

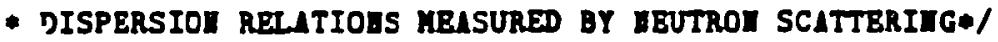

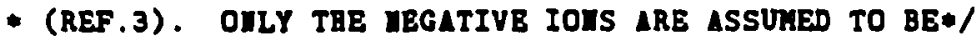

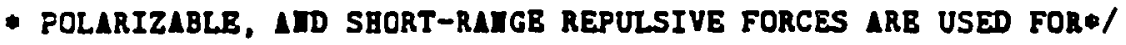

- teE fIRSt AID SECOTd teIgBBORS. THE FREQUETCY SPECTRA*/

- ueigated by tab squares of tab auplitude vectors uere*/

- COMPUTED SEPARATEIY FOR BERYLIUT ATD OXYGEI ITD USED TO*/

- calculate separate scatteriug laus yitg gasket. tBe*/

- SCATTERIMG laUS veRg TBEI COMBIJED AID ADJUSTED TO BE*/

- USED UITE TEB BERYLIUN FREE-ATOM CROSS SECTIOA. TEE*/

- OXYGEI FREB-ATOH CROSS SECTIOI ALS BEED PROVIDED FOR USE*/

- UITH THE SBORT-COLLISION-TIMB APPROXIMATIOI (SCT). TBUS,*/

* the tHerhal choss SECTIOI COMPUTED FROX BITHER S(ALPHA,BETA)*/

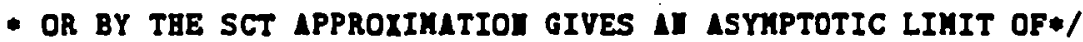

- approtimately 6.15+3.75 BARIS. TBB ELASTIC PART OF TBE*/

- scatteritg yas calculated using the average of the debye-haller*/

- factors for beryliun atd oxygej. */

* 1

- REFEREJCES*/

- -

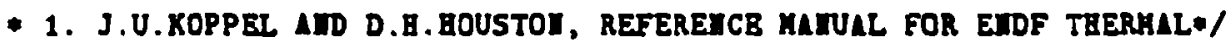

- meutrol scatteritg data, gemeral atomic report ga-8774*/

- REVISED ATD RETSSUED AS EDDF-269 BY TBB mational juctear*/

- data CEUtBa, juLy 1978.*/

- 2. G.bORGoINOVI, LATIICB DYMAMICS AID EEUTROI SCATTERIJG OF BEO, $*$

- crmbal ltokic report gl-8768 (1968). /

- 3. R.M. BRUgGER, X. A.STROIG, IID J.M.CARPEITER, J.PBYS.CBEM.*/

- SOLIDS $28,240(1967) \ldots$

* 4. R.B. MACFARLAIB, IEV TBERHLL IEUTROU SCATTERIJG FILES FOR */

* EIDF/B-VI REIEASE 2, LOS aLAMOS MATIOTAL LABORLTORY REPORT */

- LA-12639-rS (TO bB PUBLisged). *

$* 1$

/

STOP

STOP

We chose to use the same $\alpha$ and $\beta$ limits as for Be. 
In this calculation, the $\alpha$ values for the secondary scatterer were transformed by the atomic weight ratio of the two atoms. This allowed us to add the $S(\alpha, 3)$ contribution for $\alpha_{i}$ from $\mathrm{Be}$ in $\mathrm{BeO}$ to the contribution for $\alpha_{i}$ from $\mathrm{O}$ in $\mathrm{BeO}$ with only a. cross-section weighting. The resulting $S(\alpha, \beta)$ is intended to be used with the beryllium cross sections.

The phonon spectrum for $\mathrm{Be}$ in $\mathrm{BeO}$ is shown in Figure 17.

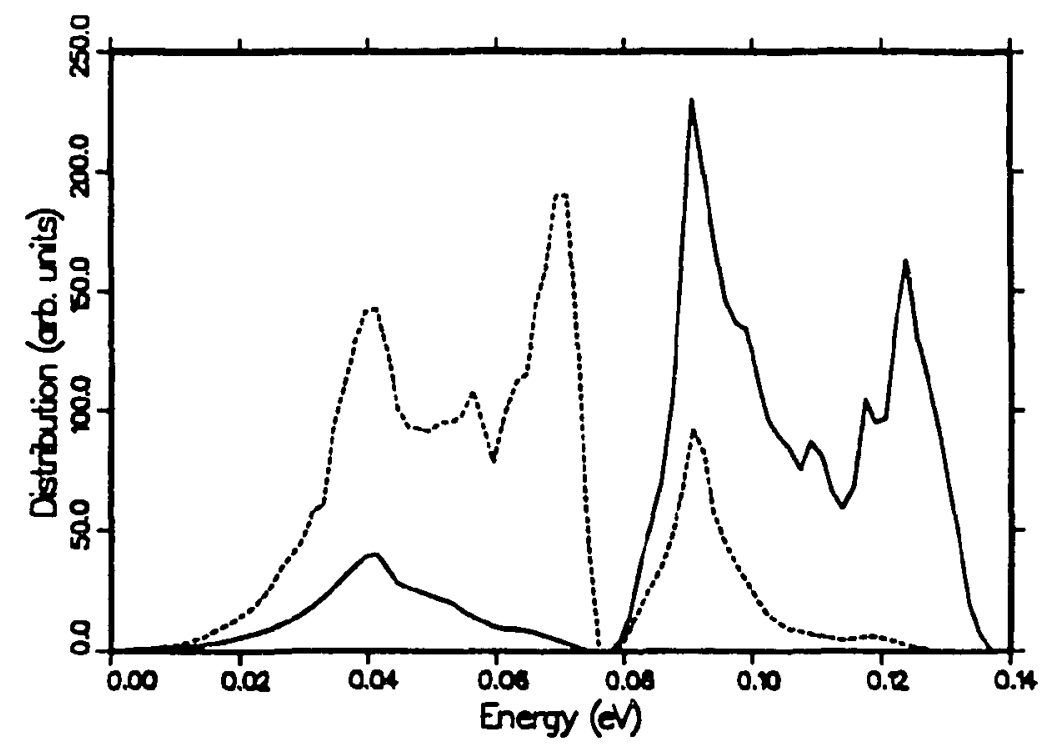

Figure 17: The frequency spectra $\rho(\epsilon)$ used for $\mathrm{Be}$ in $\mathrm{BeO}$ (solid) and $\mathrm{O}$ in $\mathrm{BeO}$ (dashed).

Figures 18 through 21 show some of the features of $S(\alpha, \beta)$ and compare the new evaluation to the old one. The differences seen here are even smaller than those seen for Be.

Figures 22 through 28 show the integrated cross sections and secondary neutron spectra for BeO. Once again, except for fixing the error in the coherent elastic scattering, there is no real improvement obtained with the new evaluation.

A table of effective temperatures and Debye-Waller factors for both constituents is given at the end of this section. The average of the Debye-Waller factors was used in computing the coherent elastic scattering for $\mathrm{BeO}$. 


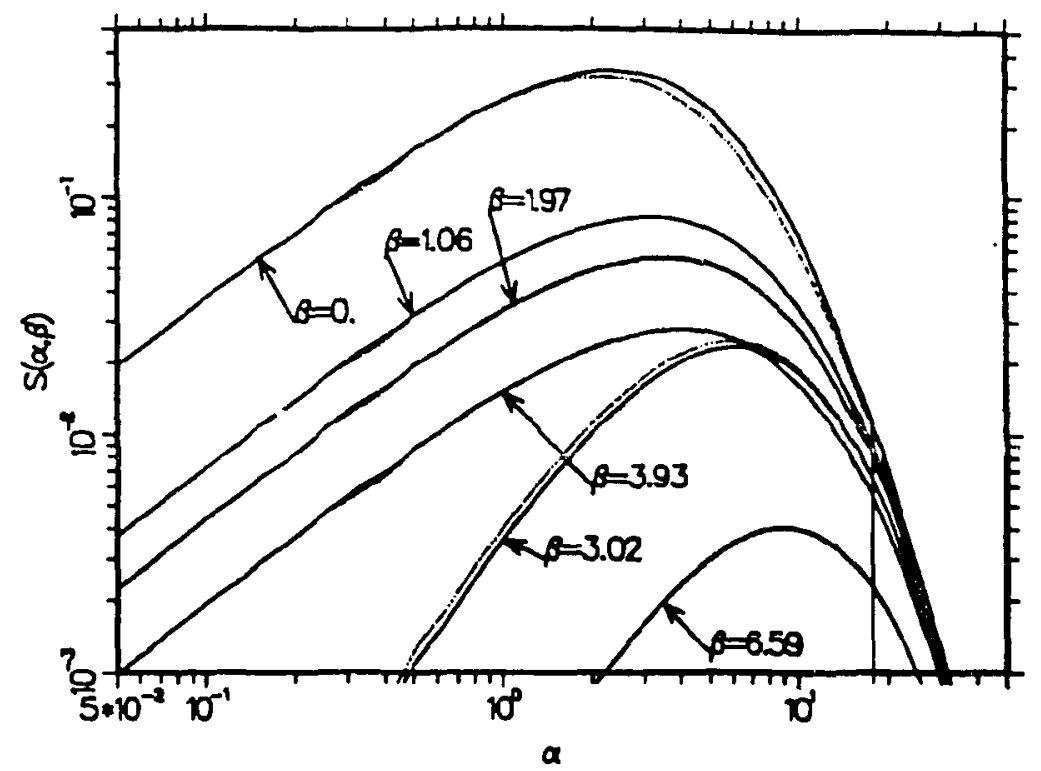

Figure 18: $S(\alpha, \beta)$ vs $\alpha$ for several $\beta$ values in $B e O$ at a temperature of $206 \mathrm{~K}$ emphasizing the low- $\alpha$ side of the function. The solid lines are the results of this calculation, and the dashed lines are for ENDF/B-VI.0.

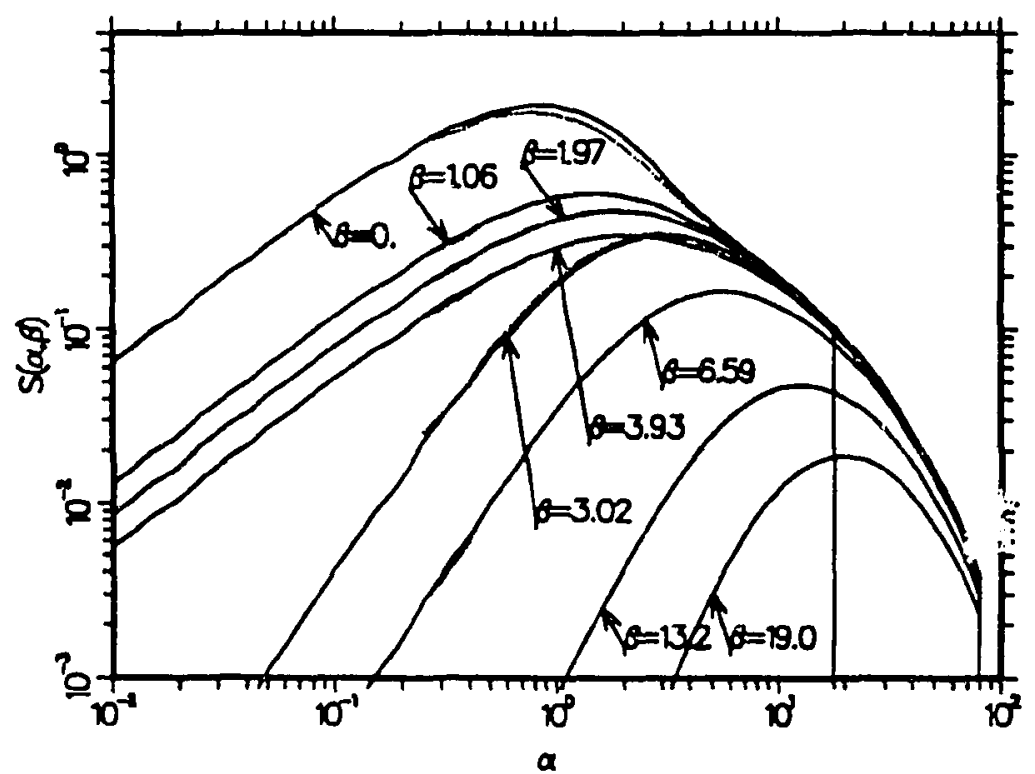

Figure 19: $S(\alpha, \beta)$ vs $\alpha$ for several $\beta$ values in $\mathrm{BeO}$ at a temperature of $1200 \mathrm{~K}$. The solid lines are the results of this calculation, and the dashed lines are for ENDF/B-VI.0. The effects of the new evaluation are similar to those seen at $296 \mathrm{~K}$. 


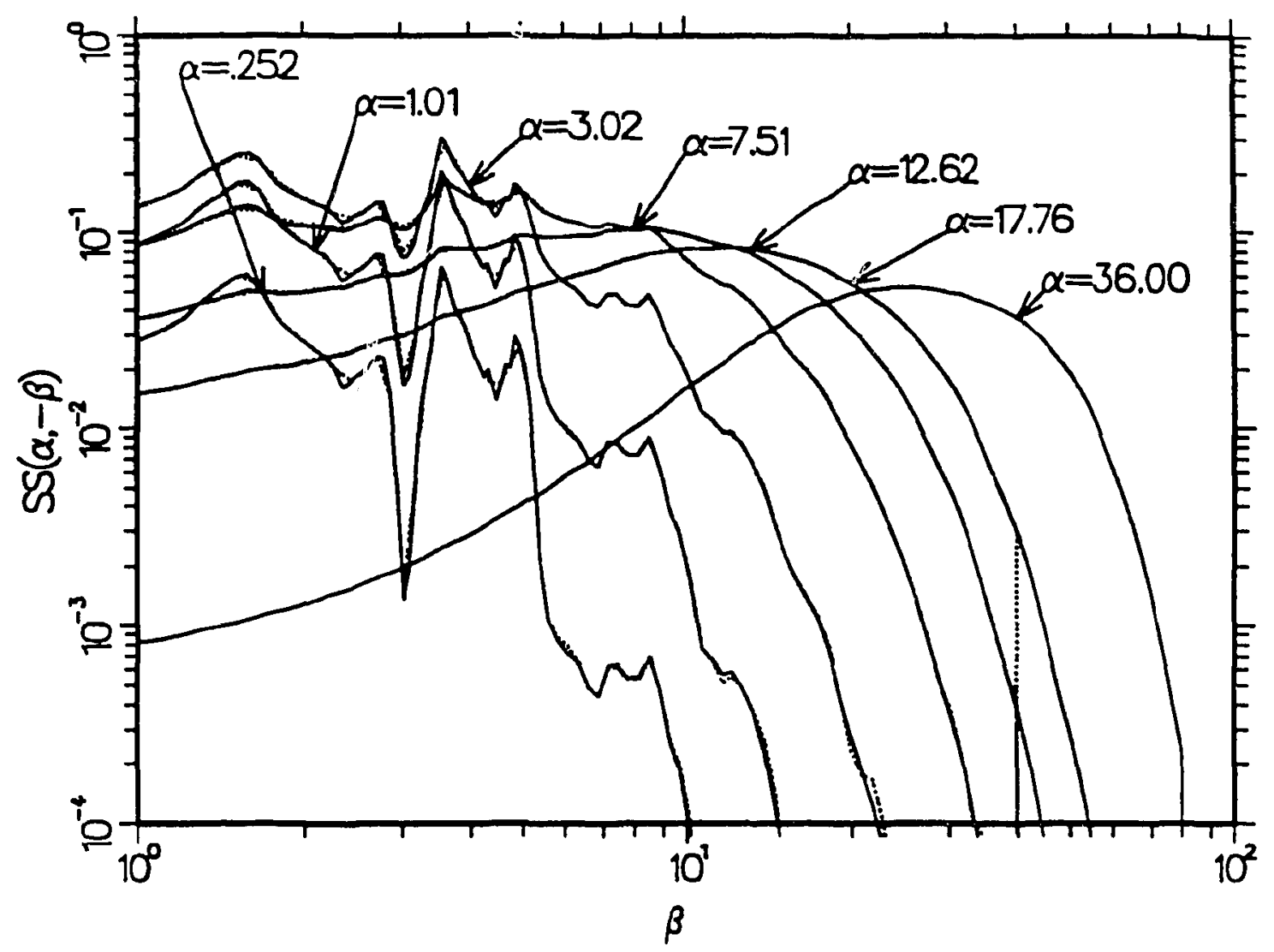

Figure 20: $\mathcal{S}(\alpha,-\beta)$ vs $\beta$ for several $\alpha$ values in $\mathrm{BeO}$ at a temperature of $296 \mathrm{~K}$. The solid lines are the results of this calculation, and the dashed lines are for ENDF/B-VI.0. The finer $\beta$ grid used in the new evaluation does a better job of following the peaks and valleys resulting from features in $\rho(\beta)$. 


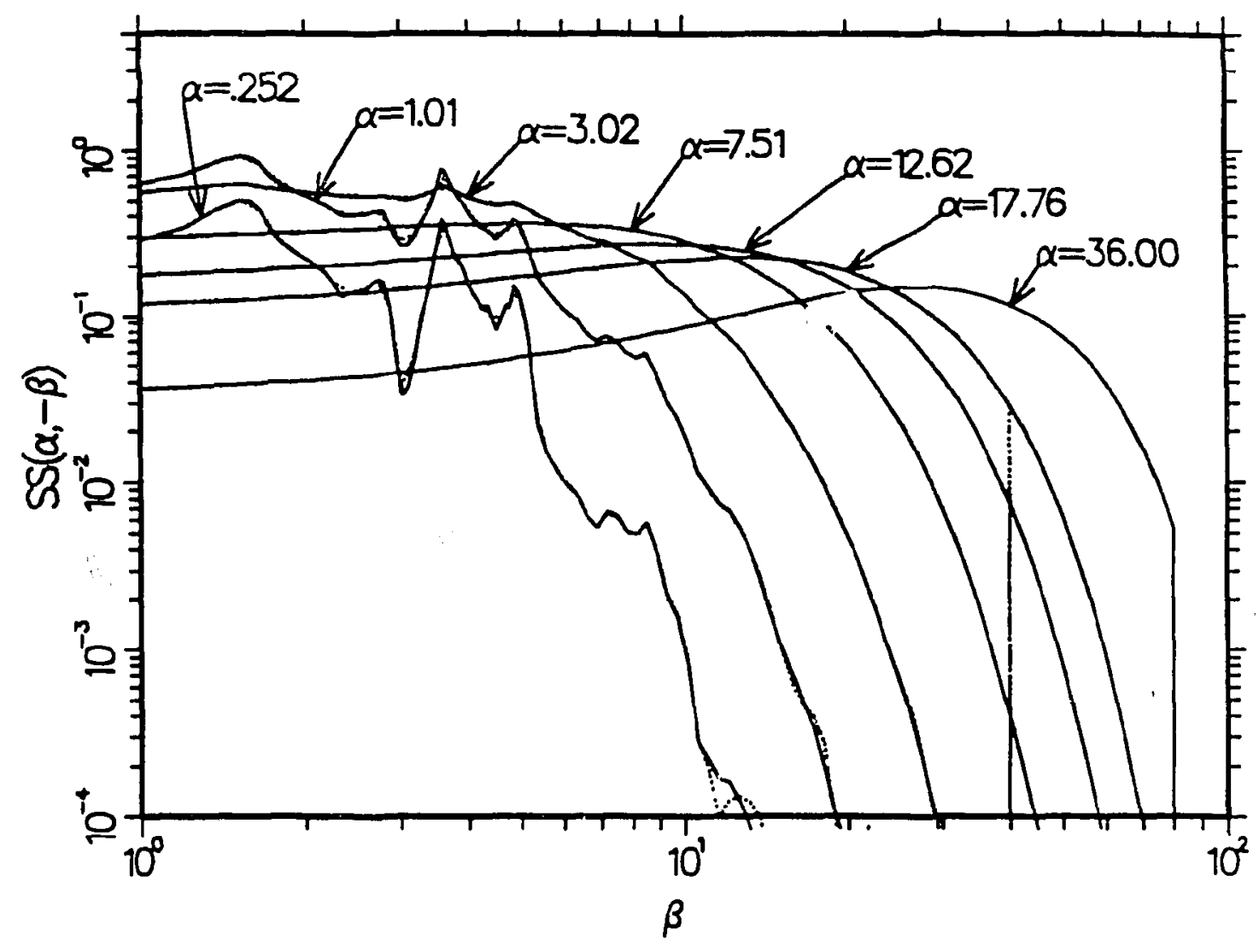

Figure 21: $\mathcal{S}(\alpha,-\beta)$ vs $\beta$ for several $\alpha$ values in $\mathrm{BeO}$ at a temperature of $1200 \mathrm{~K}$. The solid lines are the results of this calculation, and the dashed lines are for ENDF/B-VI.0. The comparisons show the same effects seen at $296 \mathrm{~K}$, except the peaks and valleys are somewhat smoothed out at this high temperature. 


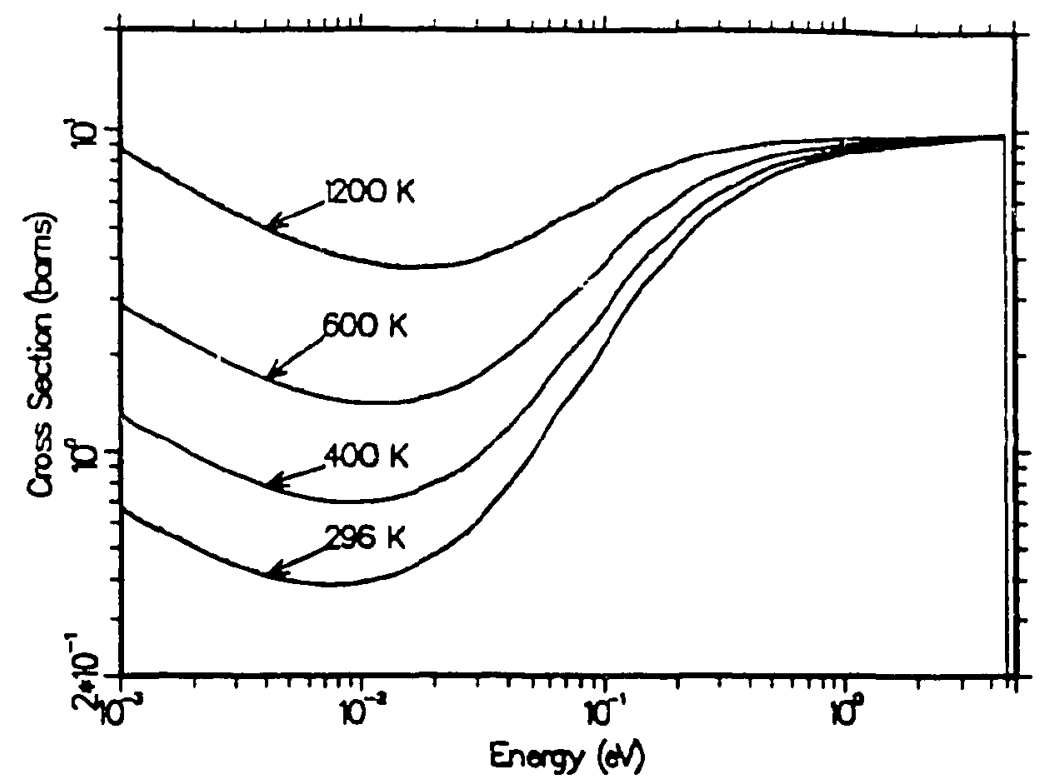

Figure 22: The inelastic cross section for $\mathrm{BeO}$ at four temperatures emphasizing the low-energy range. The dashed curves are for the ENDF/B.VI.0 evaluations, and there is very little difference seen.

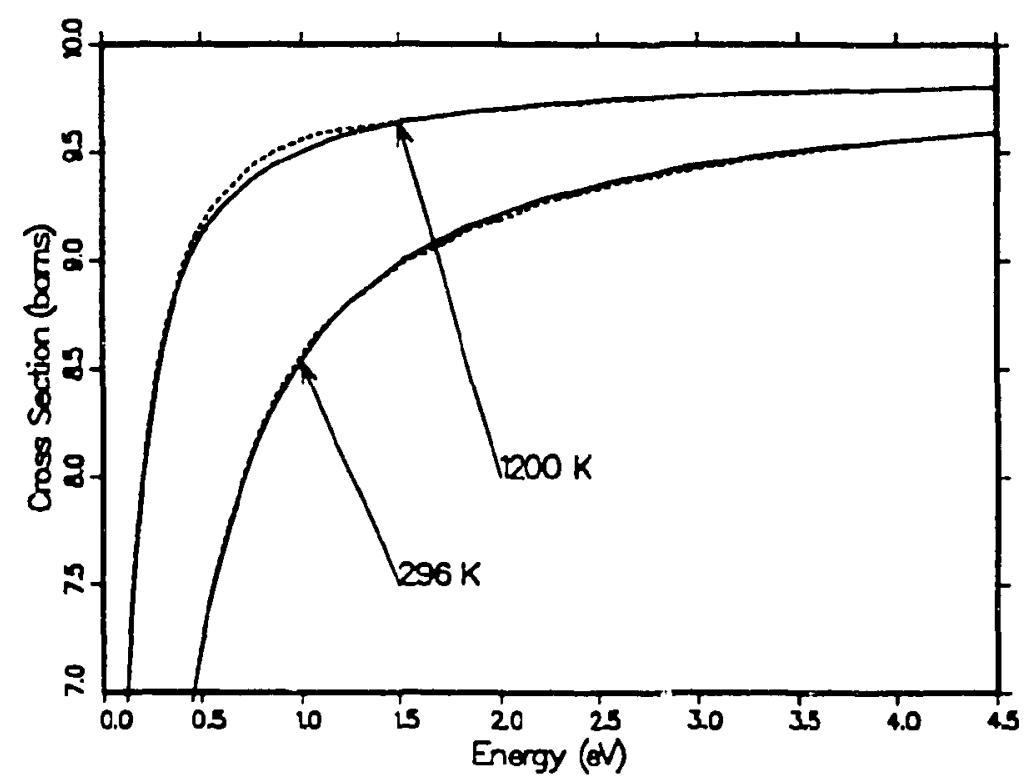

Figure 23: The inelastic cross section for $\mathrm{BeO}$ at temperatures of 296 $\mathrm{K}$ and $1200 \mathrm{~K}$ emphasizing the high-energy range. The dashed curves are for the ENDF/B-VI.0 evaluations. 


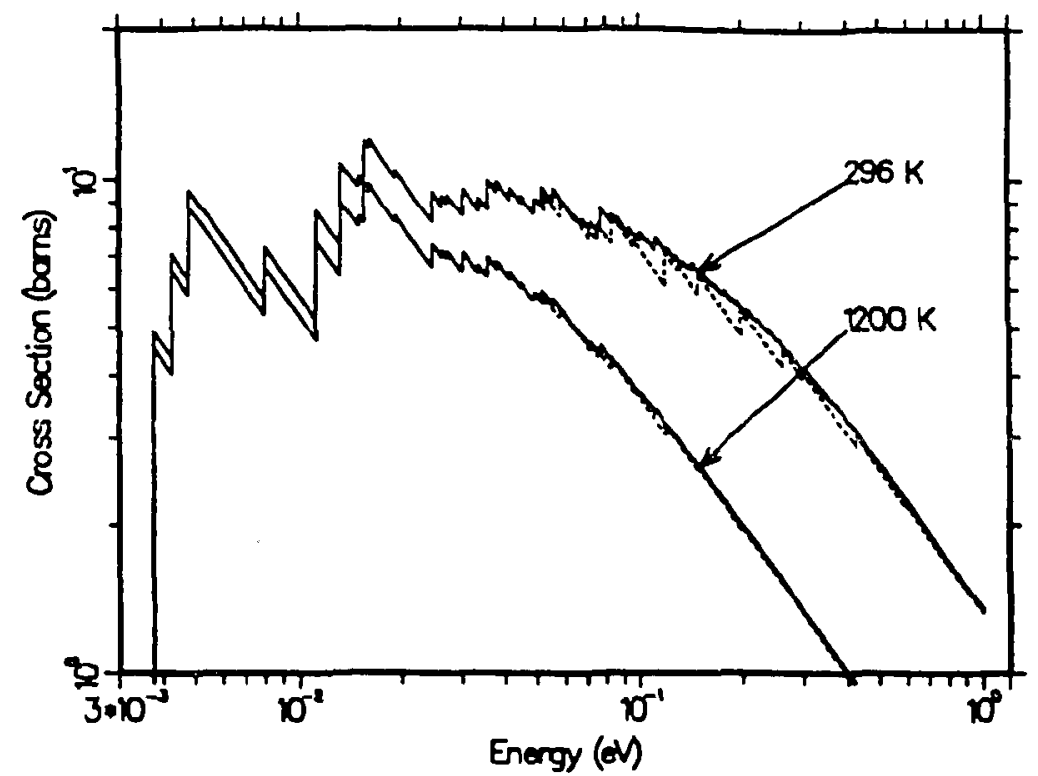

Figure 24: The coherent elastic cross section for $\mathrm{BeO}$ at temperatures of $296 \mathrm{~K}$ and $1200 \mathrm{~K}$ showing the Bragg peaks. The dashed curves are for the ENDF/B-VI.0 evaluations. Note the problem in the old evaluation near $.1 \mathrm{eV}$ for $296 \mathrm{~K}$.

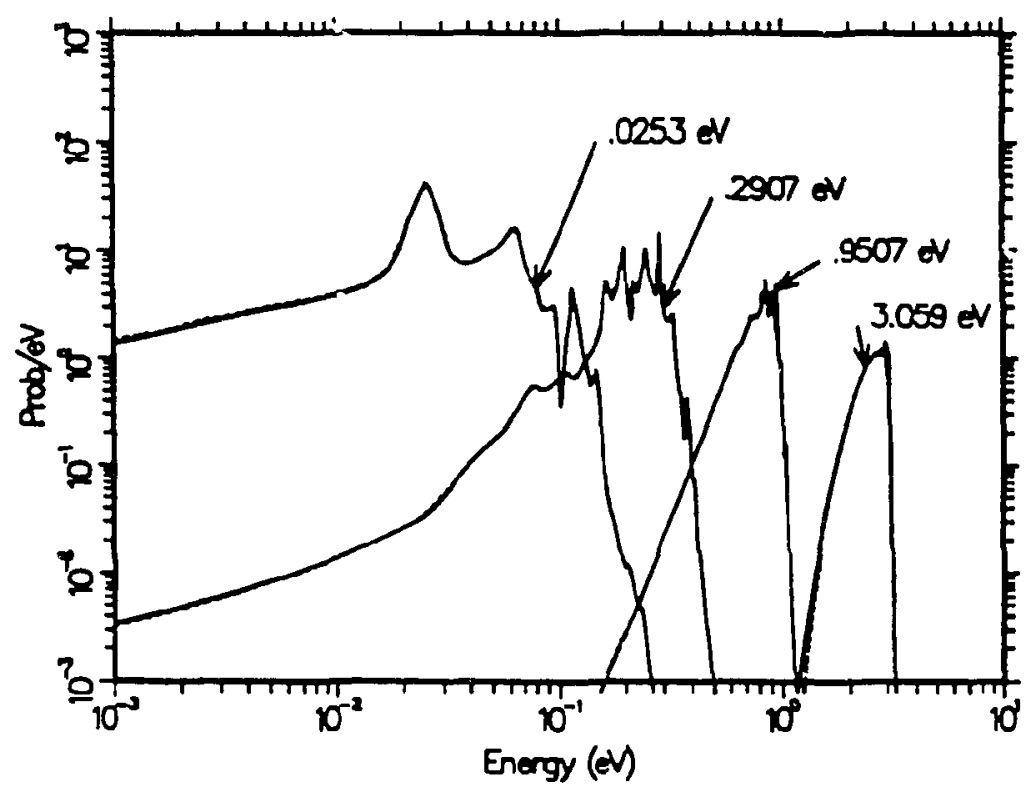

Figure 25: Secondary neutron spectra for $\mathrm{BeO}$ for several incident energies at a temperature of $296 \mathrm{~K}$. The dashed curves are for the ENDF/B-VI.0 evaluation. 


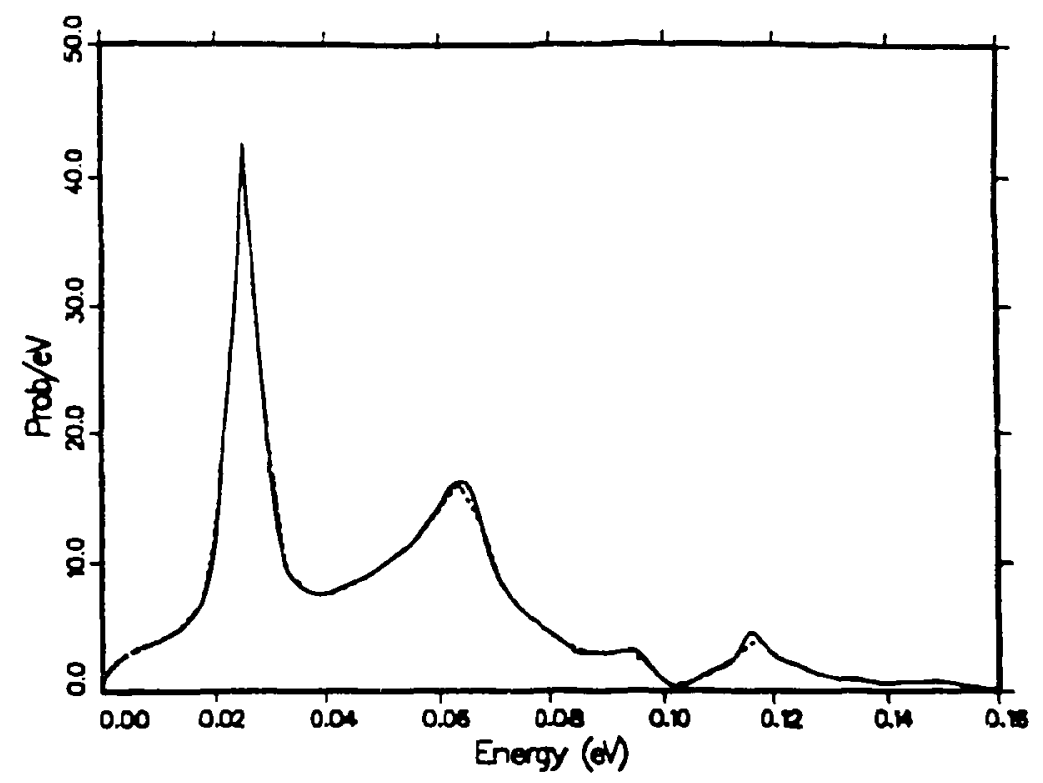

Figure 26: Expanded view of the secondary neutron spectrum at $.0253 \mathrm{eV}$ for $\mathrm{BeO}$ at a temperature of $296 \mathrm{~K}$. The dashed curve is the ENDF/B-VI.0 evaluation.

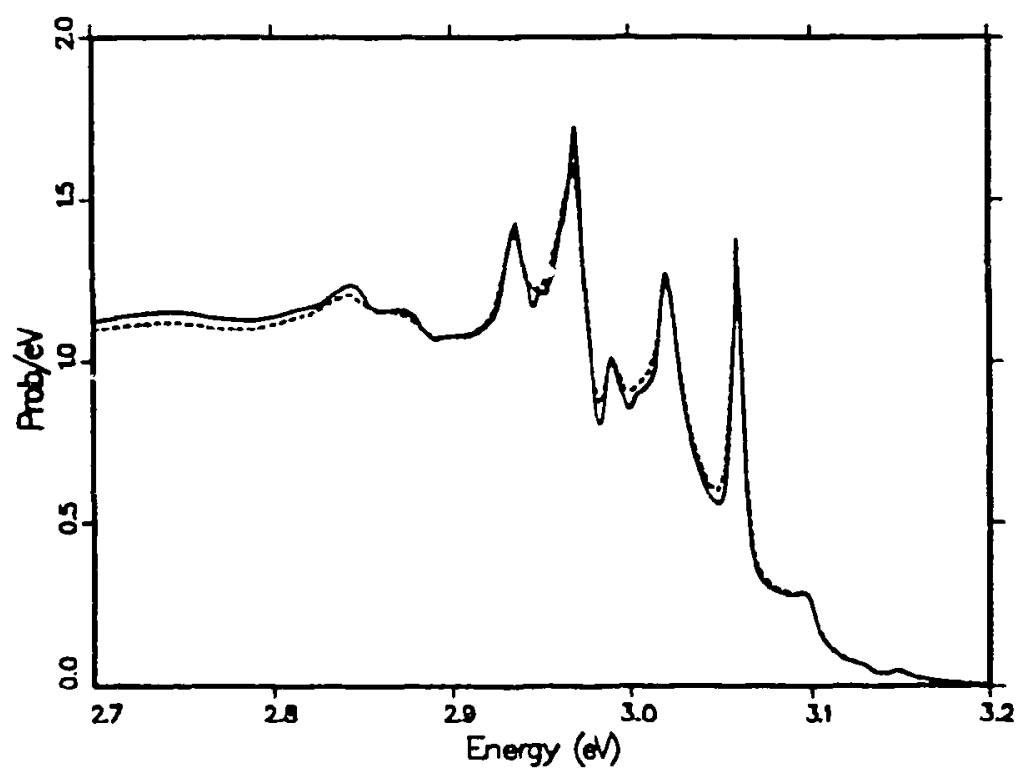

Figure 27: Expanded view of the secondary neutron spectrum at $4.46 \mathrm{eV}$ for $\mathrm{BeO}$ at a temperature of $296 \mathrm{~K}$. The dashed curve is the ENDF/B-VI.0 evaluation. 


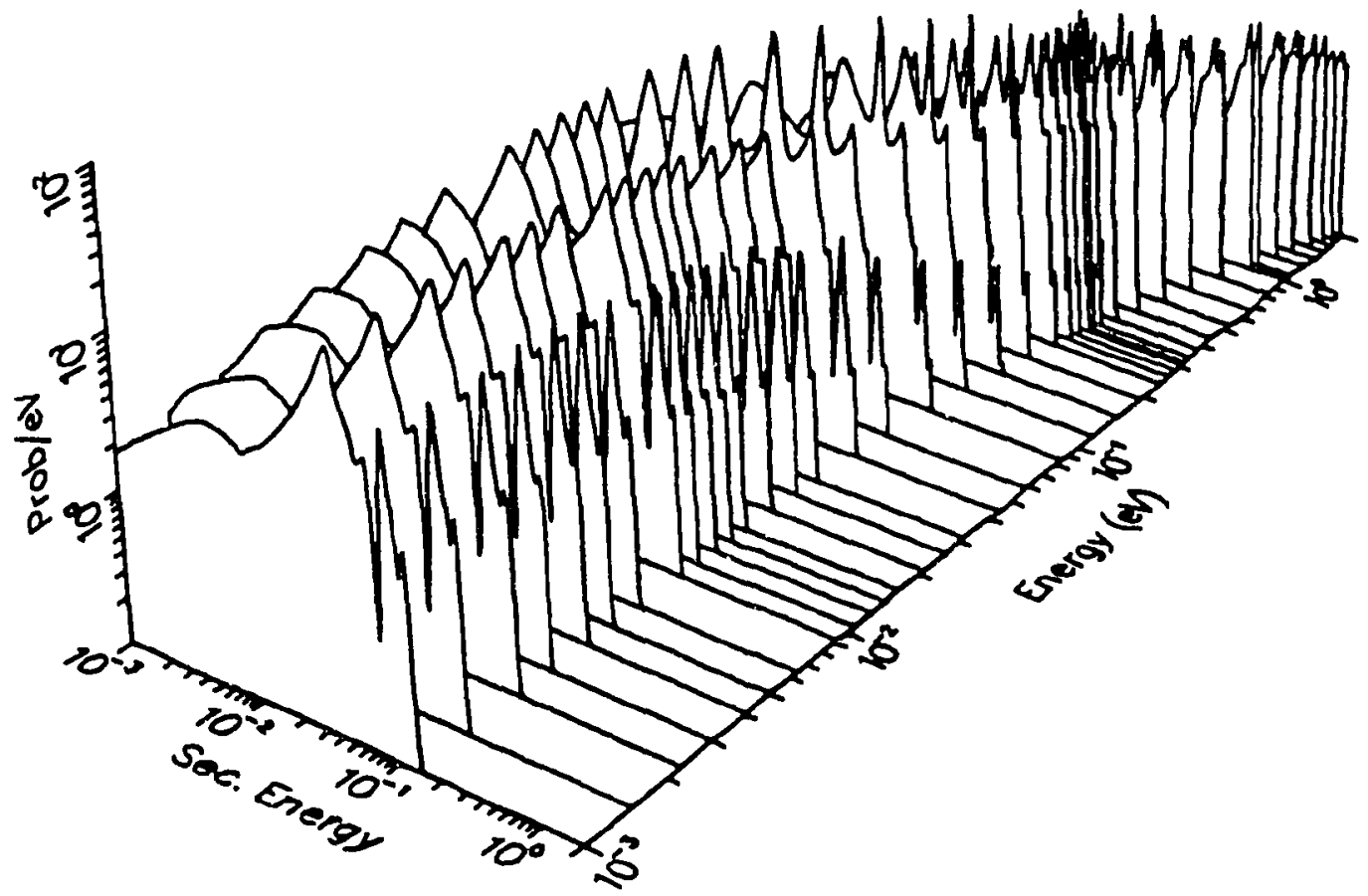

Figure 28: Perspective view of the secondary neutron spectra from thermal inelastic scattering from $\mathrm{BeO}$ at $296 \mathrm{~K}$. 
Table 3: Table of Debye-Waller Integrals and Effective Temperatures for $\mathrm{BeO}$

\begin{tabular}{ccccc}
\hline $\begin{array}{c}\text { Temperature } \\
(\text { deg K) }\end{array}$ & $\begin{array}{c}\text { Be in BeO } \\
\text { Debye-Waller } \\
\text { Int. }\left(\mathrm{eV}^{-1}\right)\end{array}$ & $\begin{array}{c}\text { Be in BeO } \\
\text { Eff. Temp. } \\
\text { (deg K) }\end{array}$ & $\begin{array}{c}\text { O in BeO } \\
\text { Debye-Waller } \\
\text { Int. }\left(\mathrm{eV}^{-1}\right)\end{array}$ & $\begin{array}{c}\text { O in BeO } \\
\text { Eff. Temp. } \\
\text { (deg K) }\end{array}$ \\
\hline 296 & 19.34 & 596.7 & 34.57 & 427.9 \\
400 & 23.19 & 644.2 & 43.23 & 502.9 \\
500 & 27.20 & 704.8 & 52.04 & 584.4 \\
600 & 31.40 & 775.4 & 61.10 & 671.3 \\
700 & 35.74 & 853.0 & 70.33 & 761.7 \\
800 & 40.16 & 935.5 & 79.66 & 854.3 \\
1000 & 49.17 & 1110. & 98.51 & 1044. \\
1200 & 58.32 & 1292. & 117.5 & 1237. \\
\hline
\end{tabular}




\section{GRAPHITE}

The basic physics for graphite was left unchanged from the original GA

evaluation. ${ }^{1}$ The force model used to compute the phovion spectrum 17,18 contains fnur force constants. One force constant is used to describe a nearestneighbor central force that binds two hexagonal planes together, another describes a bond-bending force in an hexagonal plane, the third is for bondstretching between nearest neighbors in the place, and the fourth corresponds to a restoring force against bending of the hexagonal plane. The force constants were evaluated numerically by performing a very precise fit to the high and low temperature specific heat and to the compressibility of reactor-grade graphite. This model was verified by comparing the computed phonon spectrum with experiment. The important changes are the extended $\alpha$ and $\beta$ grids, an updated value fcr the cross section to match the value in ENDF/B-VI, and the use of LEA.PR itself. The input deck for the LEAPR graphise run follows:

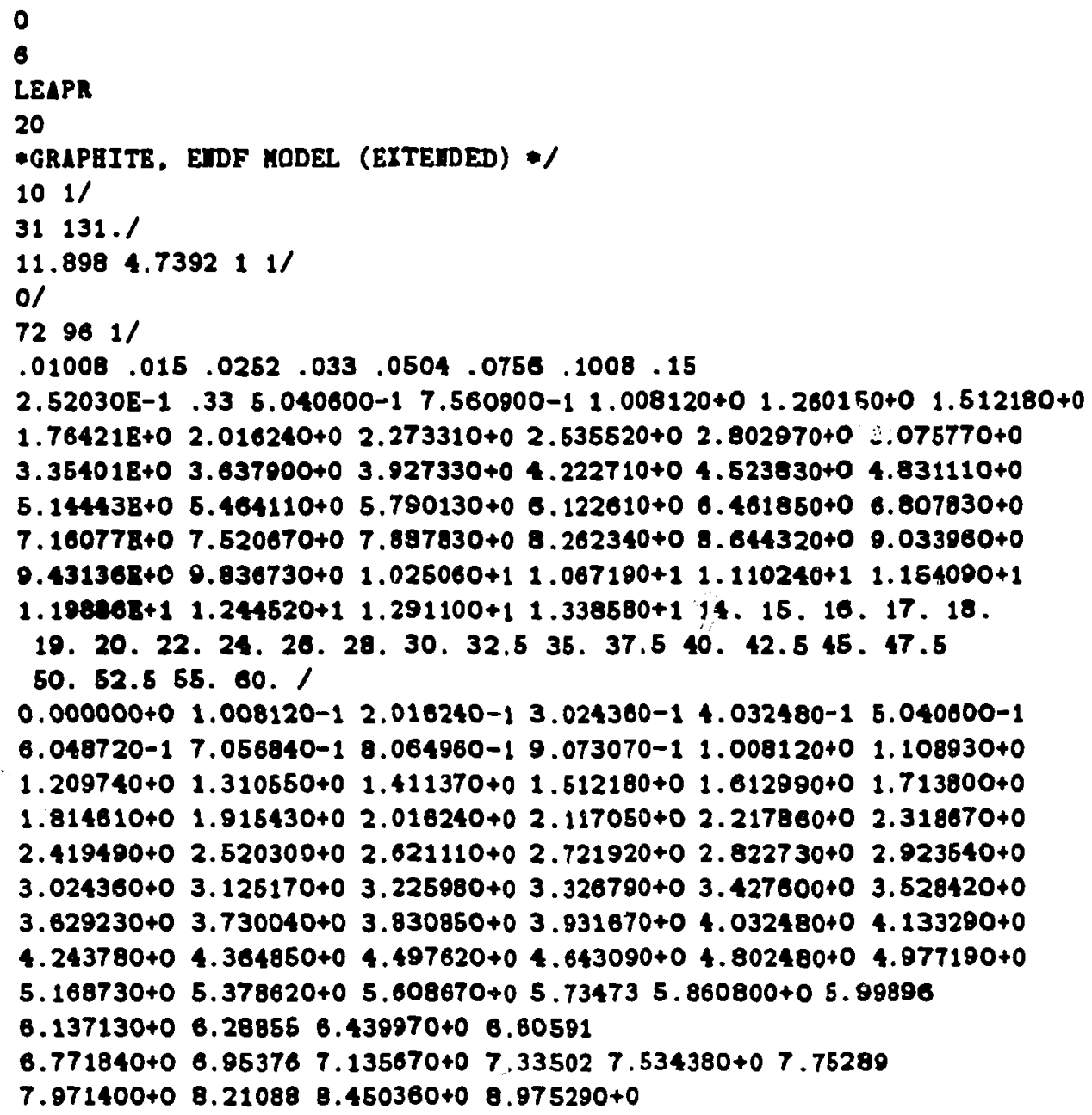




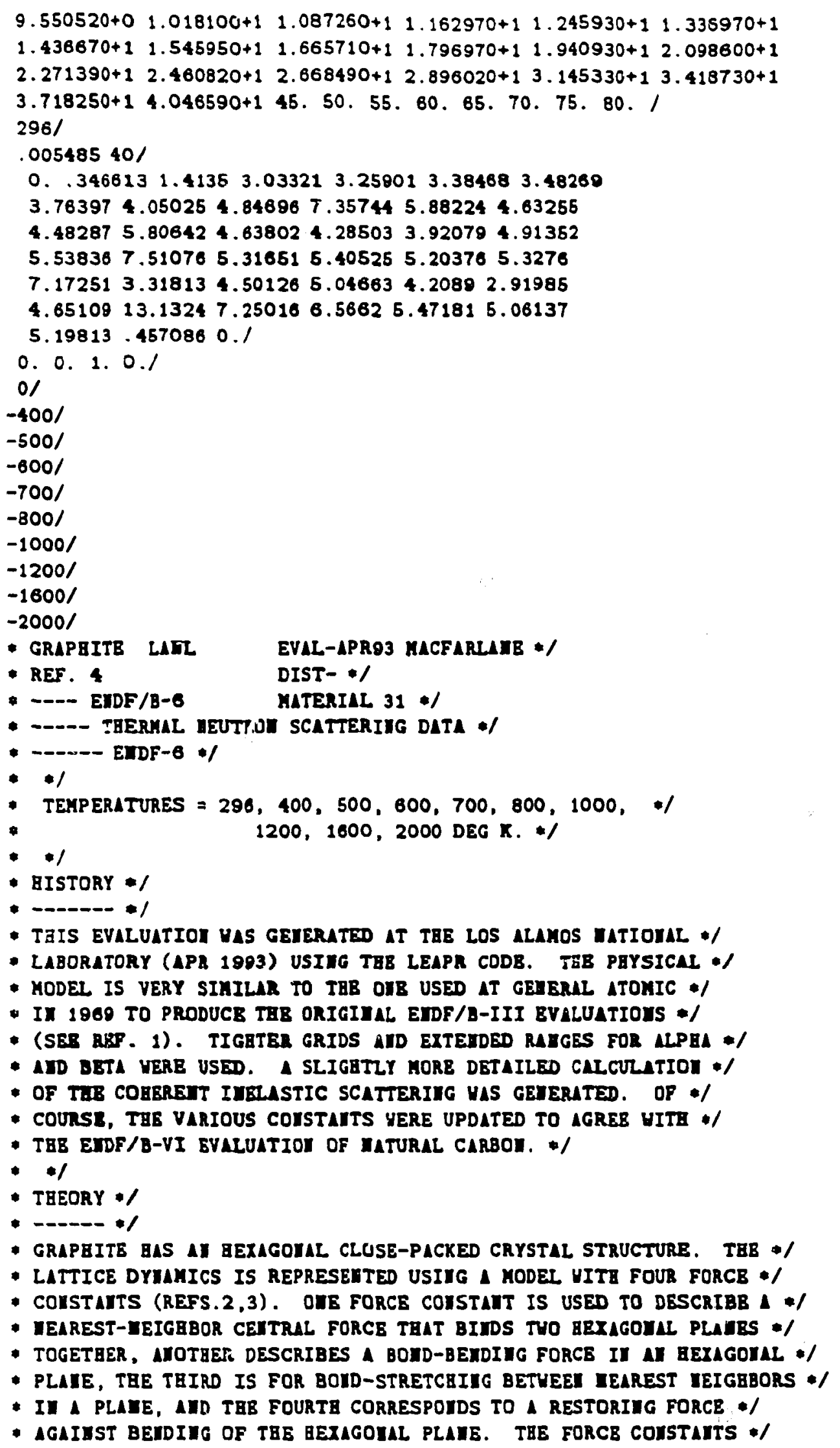




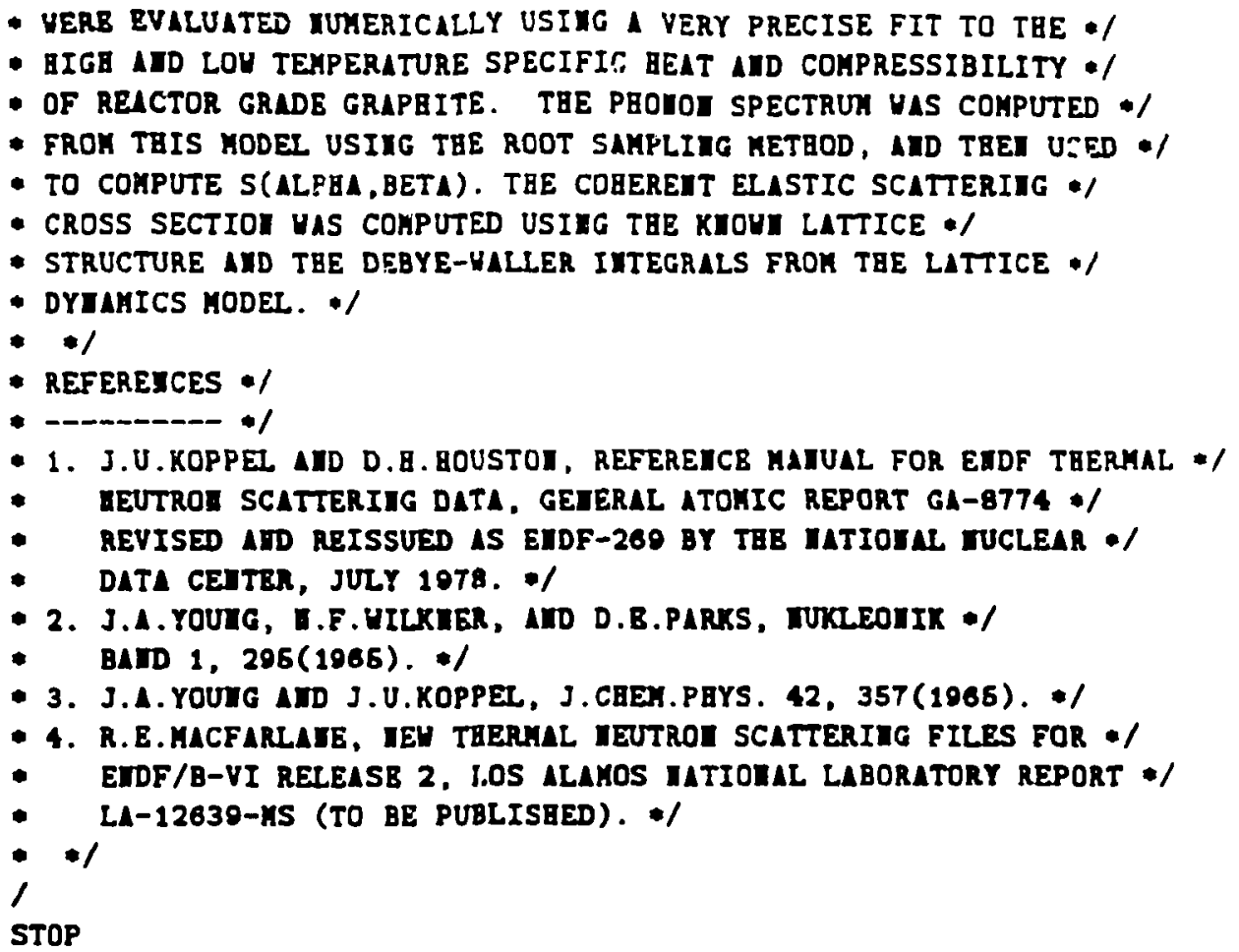

The maximum value for $\alpha$ was chosen using $\alpha_{\max }=(4 / A)\left(E_{\max } / k T\right)$, the limit for small $\beta$. The value of 60 works for energies up to $4.5 \mathrm{eV}$. It is not necessary to carry $\beta$ up to a full 160 , or more, because kinematic effects limit $\beta$ to about 52 at $4.6 \mathrm{eV}$. The value 80 used here should be adequate.

The atomic weight ratio and free cross section for carbon were taken from ENDF/B-VI. The frequency distribution was copied directly from the GASKET input in the GA report, except the last point was changed to satisfy LEAPR input restrictions (see Figure 29). As a test, the first run used IPRINT=2 to obtain a listing containing the maximum amount of information. After echoing back the infut, the code printed out $\rho(\beta), P(\beta)$, and $\mathcal{T}_{1}(\beta)$ in normalized form. It then printed out the effective temperature for the SCT approximation and the Debye-Waller factor needed for the coherent scattering cross section calculation. The break points between the phonon expansion and the SCT were also shown. LEAPR next computed the $S(\alpha, \beta)$ function for each $\alpha$. For quality control, it displayed the results of the normalization and sum rule tests. If these tests are not fairly close to unity, it may be necessary to tighten up the grids used for the calculation. Don't worry about test failures at high values of $\alpha$; the $\beta$ grid does not extend to high enough values to complete the integral over $\beta$. It also printed out values of $S(\alpha, \beta)$ and $S(\alpha, \beta)$ for both upscatter and downscatter. 


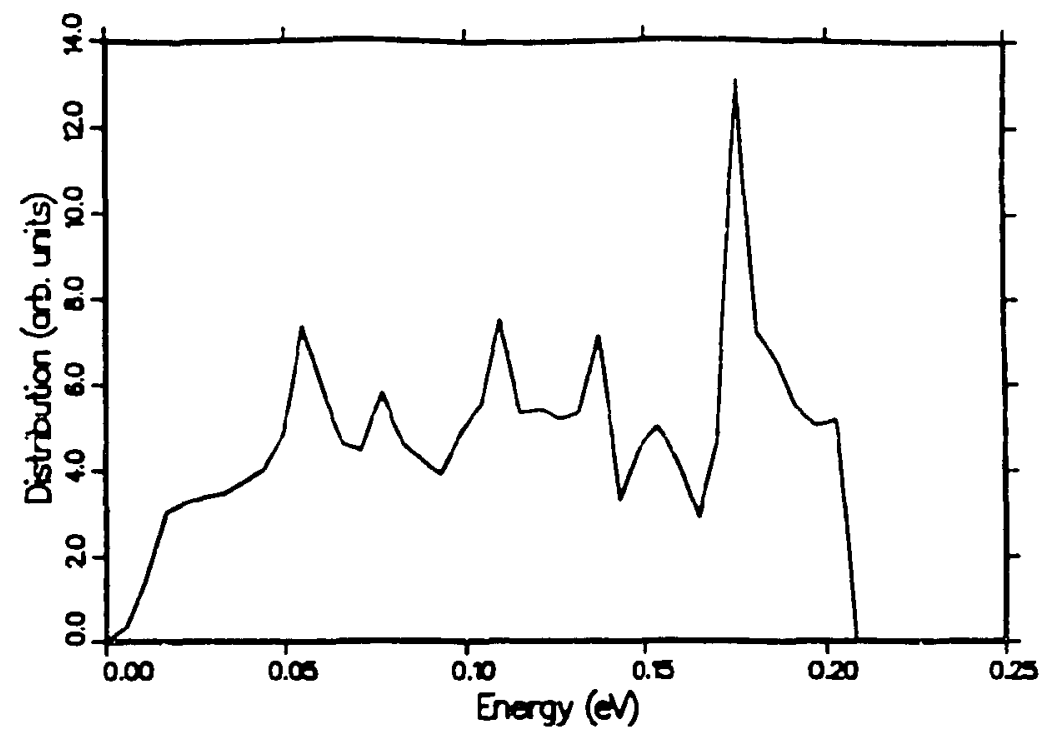

Figure 29: The phonon frequency spectrum $\rho(\epsilon)$ used for graphite.

No obvious problems with the $\alpha$ and $\beta$ grids were seen.

An examination of the final ENDF-6 output on "tape20" shows that both coherent elastic scattering ( $M F=7, M T=2)$ and incoherent inelastic scattering $(\mathrm{MF}=7, \mathrm{MT}=4)$ are included in the new evaluation. The coherent scattering extends all the way to $4 \mathrm{eV}$, which is an improvement over the original GA evaluation. Figures 30 through 33 show several views of $S(\alpha, \beta)$ for $T=296 \mathrm{~K}$ and $1200 \mathrm{~K}$ compared to the corresponding results from ENDF/B-VI.0. The first two figures show that the agreement between the new and old results is good, except that the new results extend over a wider $\alpha$ range. The second two graphs show that the old evaluation sometimes breaks down for small $S$ and that the peak near $\beta=7$ was not represented very well. The LEAPR curves are clearly more selfconsistent than the GASKET results.

The incoherent cross section computed from the new $S(\alpha, \beta)$ is shown in Fig. 34 for four temperatures. The differences seen for low incident energy are caused by the extension of the $\alpha$ grid to very low values in the new evaluation, and they are substantial. Figure 35 gives another view of the integrated inelastic cross section, emphasizing high energies. Here again, the differences are substantial above the $1 \mathrm{eV}$ cutoff used in the old evaluation. Figure 36 shows the coherent elastic cross section. 


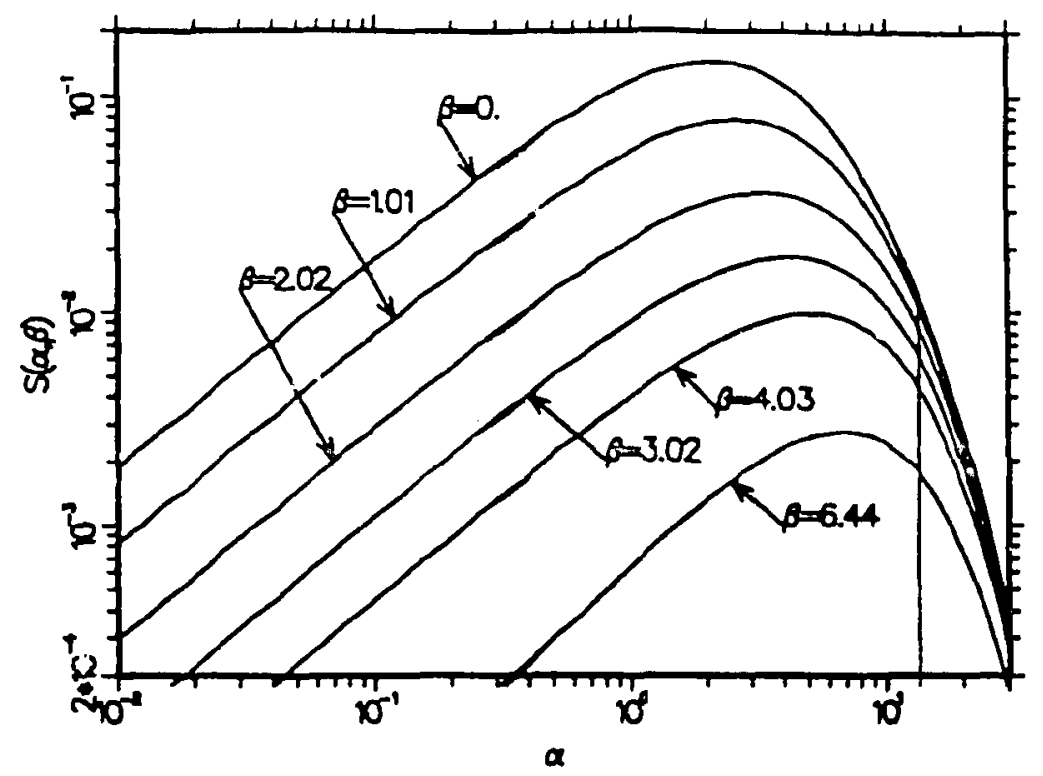

Figure 30: $S(\alpha, \beta)$ vs $\alpha$ for several $\beta$ values at $296 \mathrm{~K}$. The solid lines are the results of this calculation, and the dashed lines are for ENDF/B-VI.0. Note that the dashed lines only cover the $\alpha$ range from .25 to 13.4 .

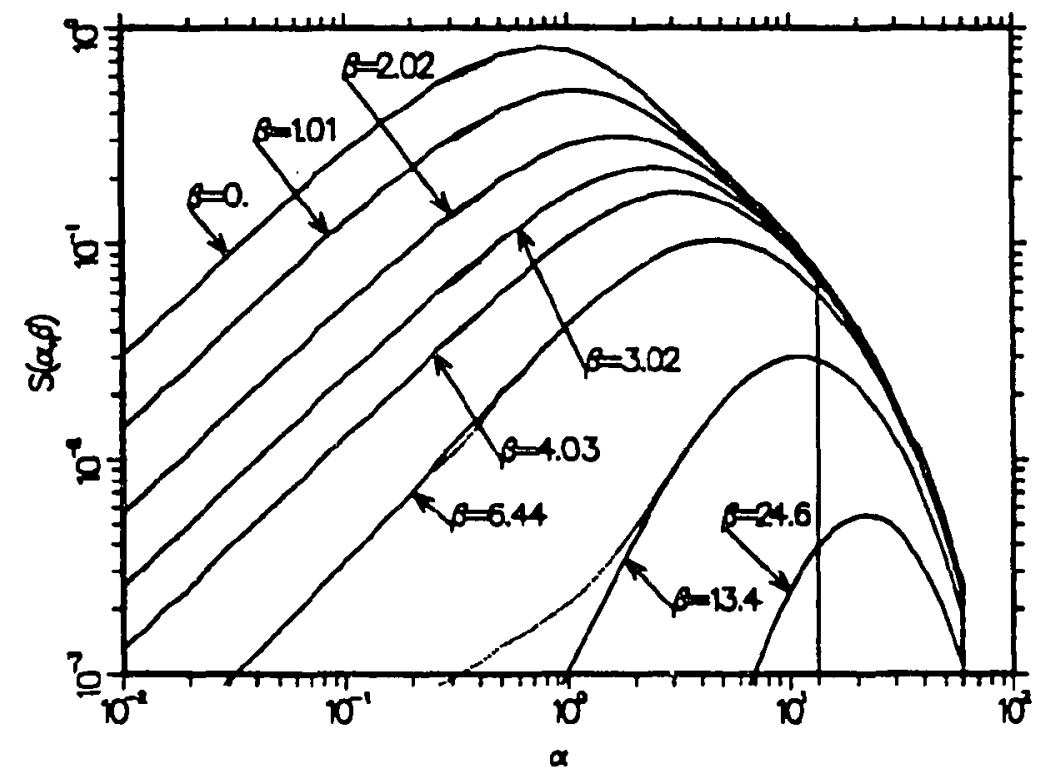

Figure 31: $S(\alpha, \beta)$ vs $\alpha$ for several $\beta$ values at $1200 \mathrm{~K}$. The solid lines are the results of this calculation. and the dashed lines are for ENDF/B-VI.0. Note that the dashed lines only cover the $\alpha$ range from .25 to 13.4 . 


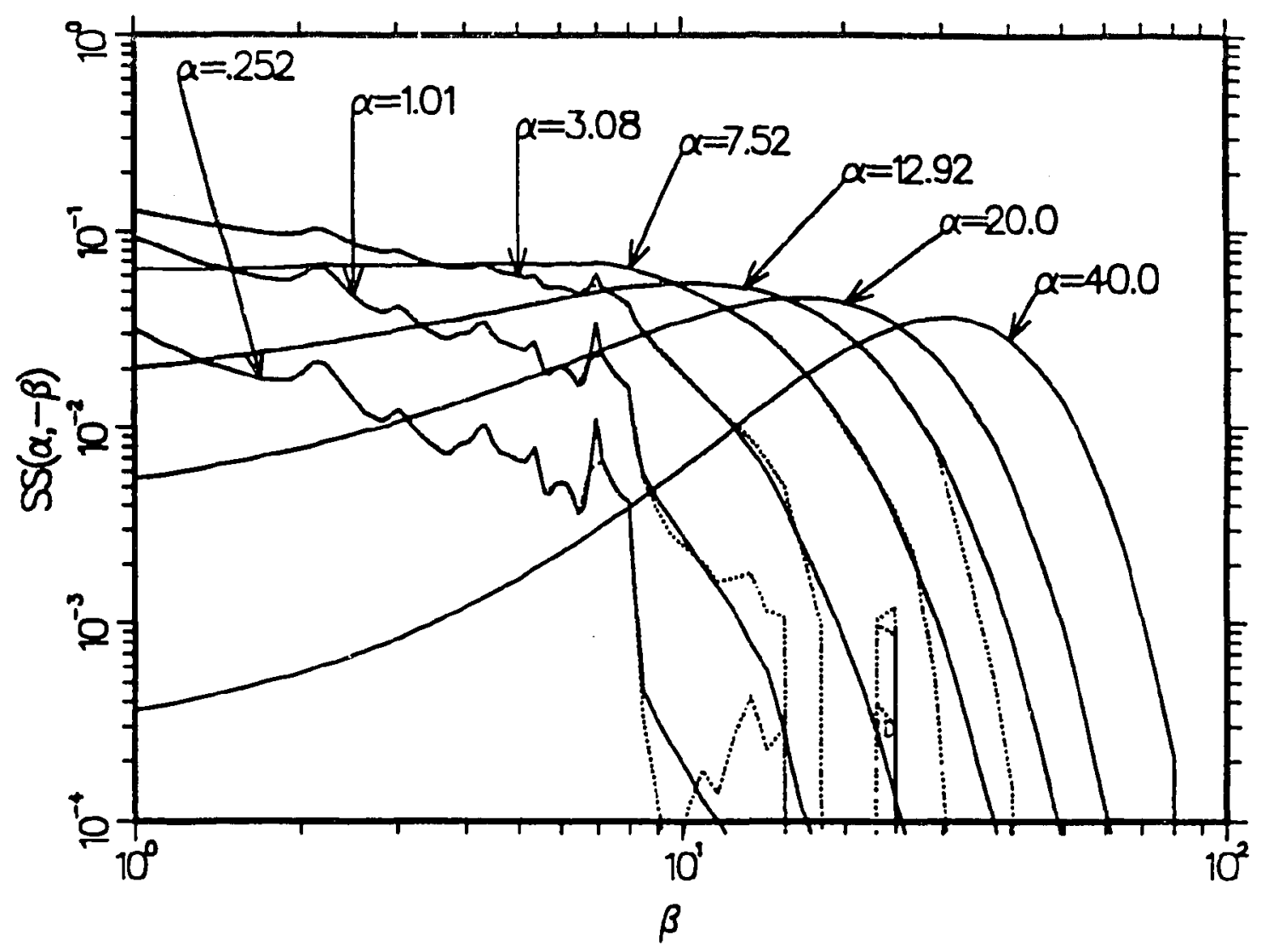

Figure 32: Asymmetric $\mathcal{S}(\alpha,-\beta)$ vs $\beta$ for several $\alpha$ values at a temperature of $296 \mathrm{~K}$. The solid lines are the results of this calculation, and the dashed lines are for ENDF/B-VI.0. 


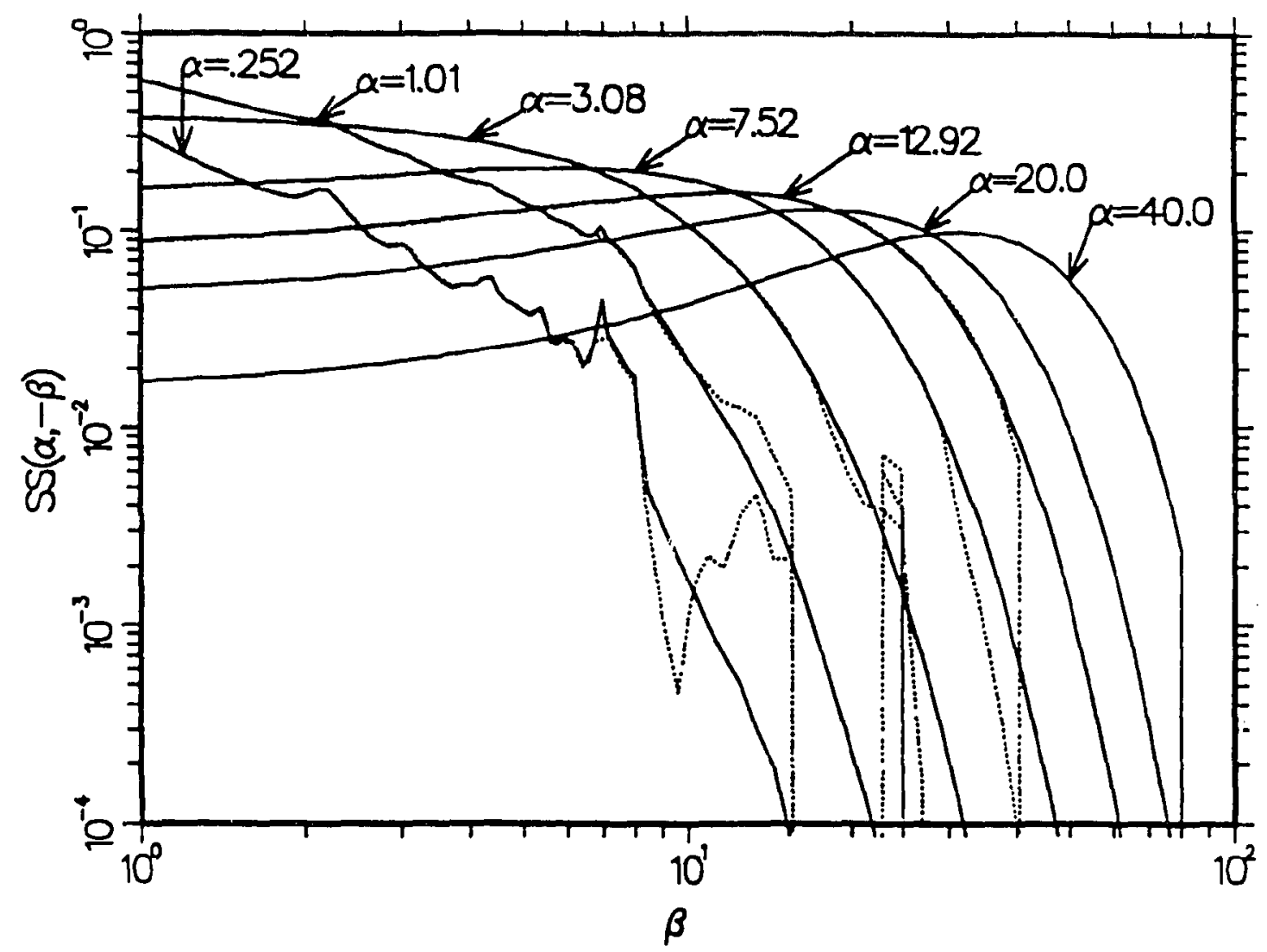

Figure 33: Asymmetric $\mathcal{S}(\alpha,-\beta)$ vs $\beta$ for several $\alpha$ values at a temperature of $1200 \mathrm{~K}$. The solid lines are the results of this calculation, and the dashed lines are for ENDF/B-VI.0. 


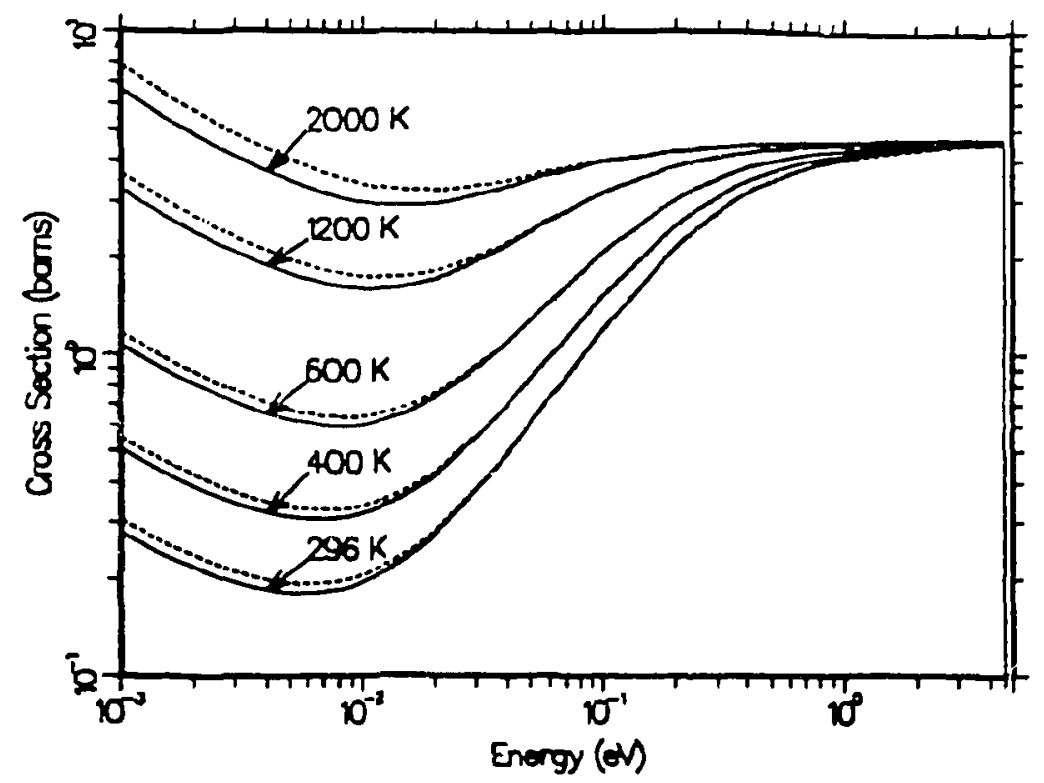

Figure 34: The incoherent inelastic cross section for graphite at temperatures from $296 \mathrm{~K}$ to $2000 \mathrm{~K}$, emphasizing low energies.

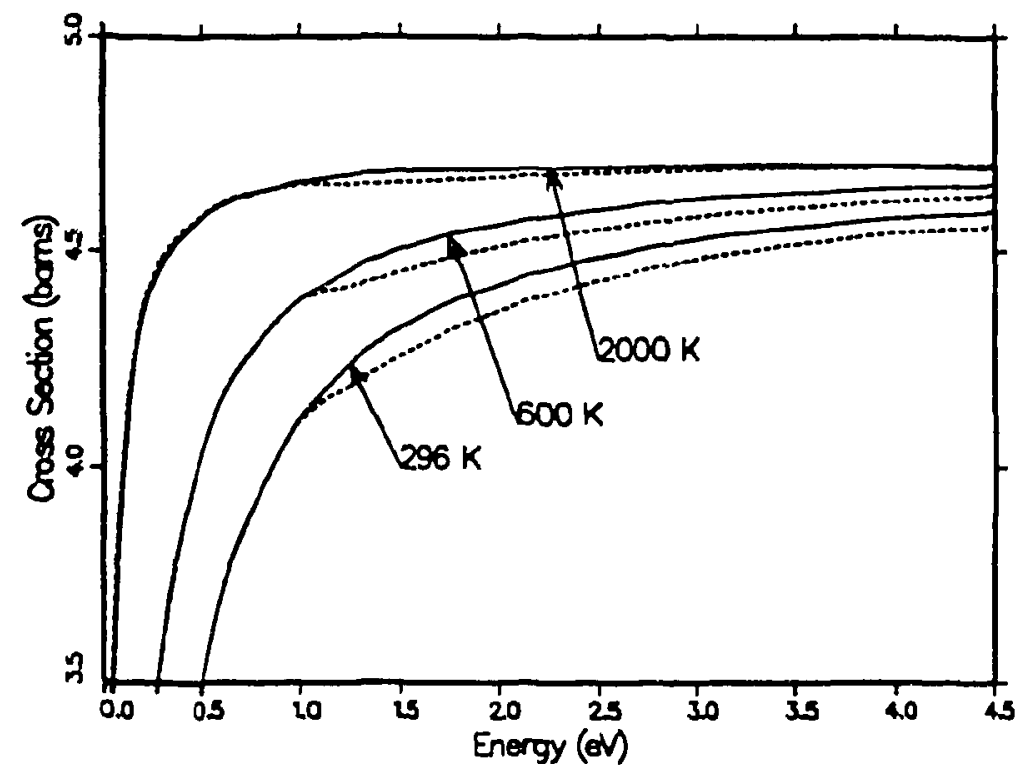

Figure 35: The incoherent inelastic cross section for graphite at temperatures from $296 \mathrm{~K}$ to $2000 \mathrm{~K}$, emphasizing high energies. The old evaluation was designed to work to only $\mathrm{l} \mathrm{eV}$. 


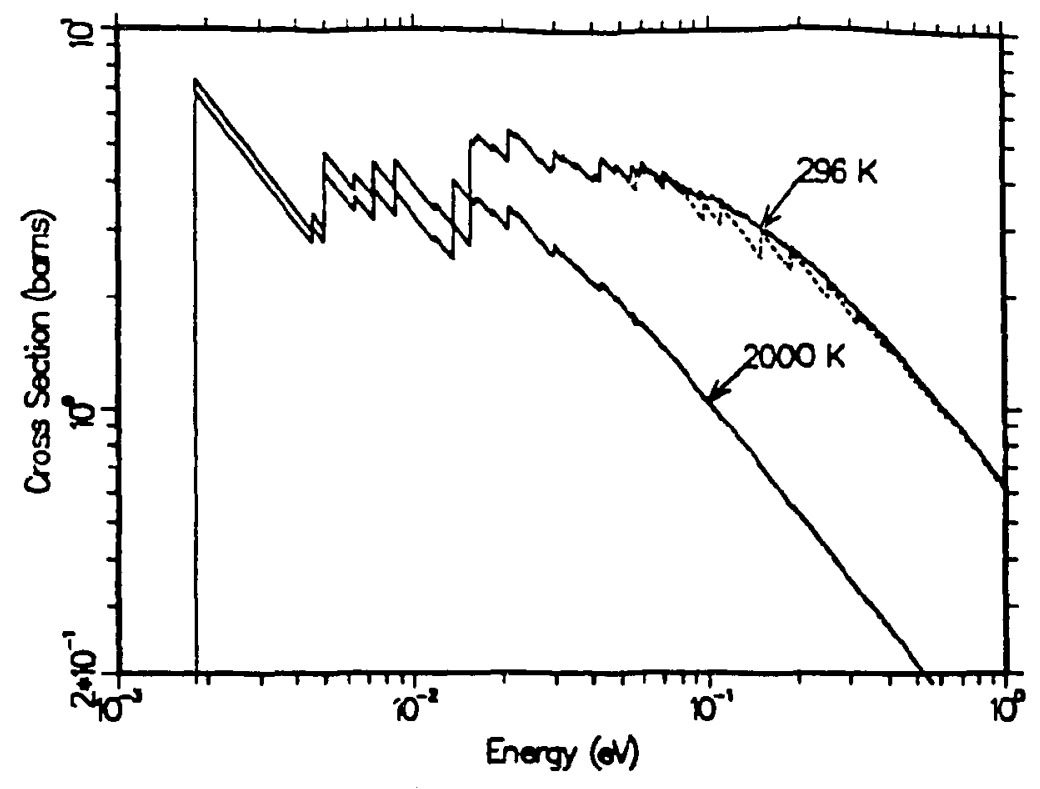

Figure 36: The coherent elastic cross section for graphite at three temperatures showing the Bragg peaks. Note that the problem in ENDF/B-VI.0 for energies above $.05 \mathrm{eV}$ has been corrected.

Figures 37 and 38 show thermal neutron emission spectra for several incident energies at two temperatures. Except for the lowest energy, the spectra from the new evaluation agree very well with those from ENDF/B-VI.0. The disagreemerits in the spectra for $E=.0005 \mathrm{eV}$ are caused by the change in the low- $\alpha$ grid as discussed above. Figures 39 and 40 show expanded views of the spectra at $296 \mathrm{~K}$ for two incident energies; they make it clear what portions of the energy range result in the substantial change in cross section visible in Figure 35. Figure 41 gives an overall view of the entire emission spectrum.

The effective temperatures for the SCT approximation and the Debye-Waller integrals used to compute the coherent elastic scattering are shown in Table 4. 


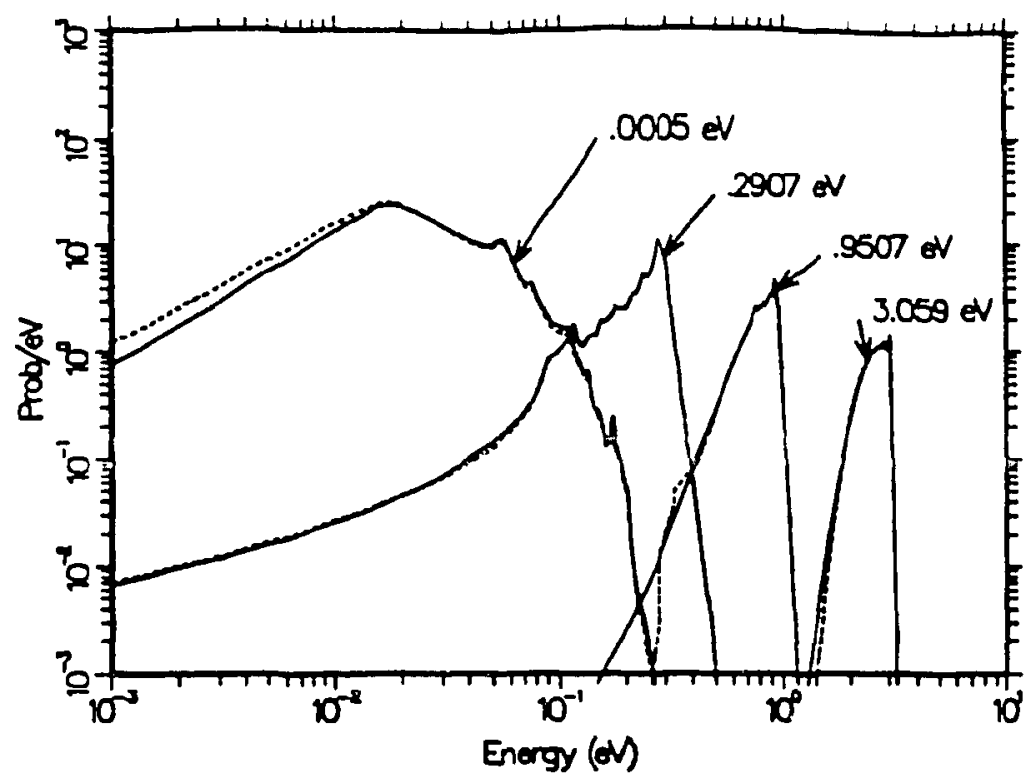

Figure 37: Spectra from thermal inelastic scattering from graphite at $296 \mathrm{~K}$ for several incident energies. The effects of the lower $\alpha$ values are evident at $.0005 \mathrm{eV}$.

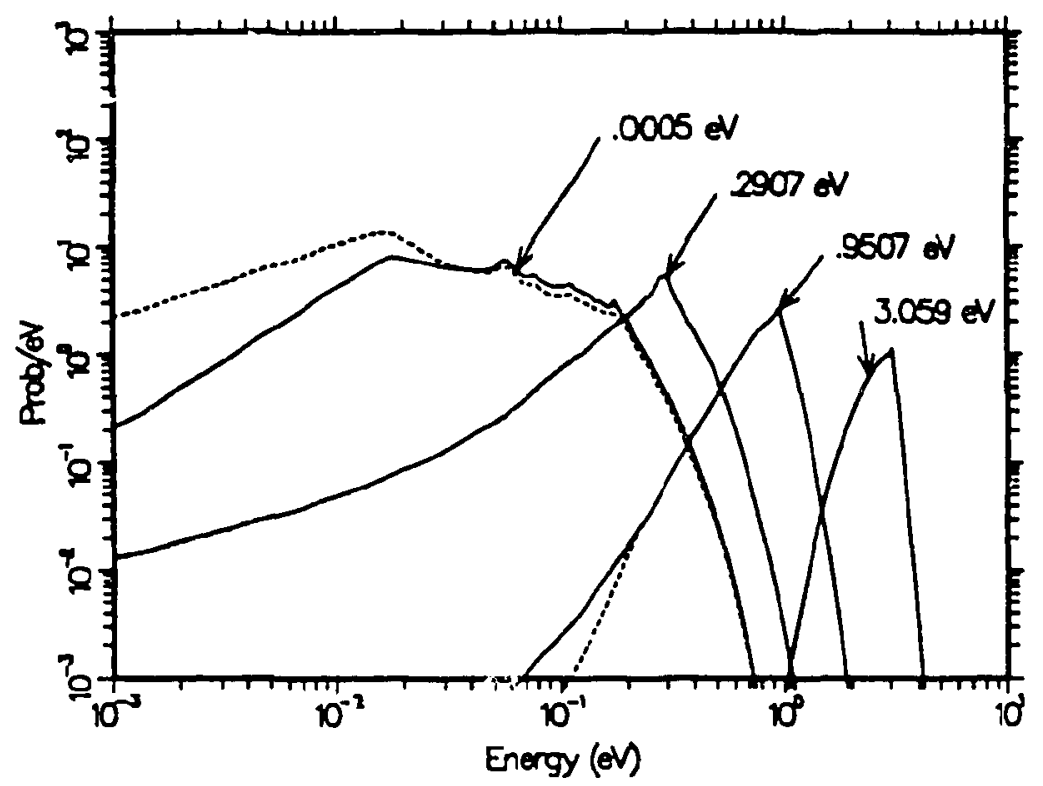

Figure 38: Spectra from thermal inelastic scattering from graphite at $1200 \mathrm{~K}$ for several incident energies. 


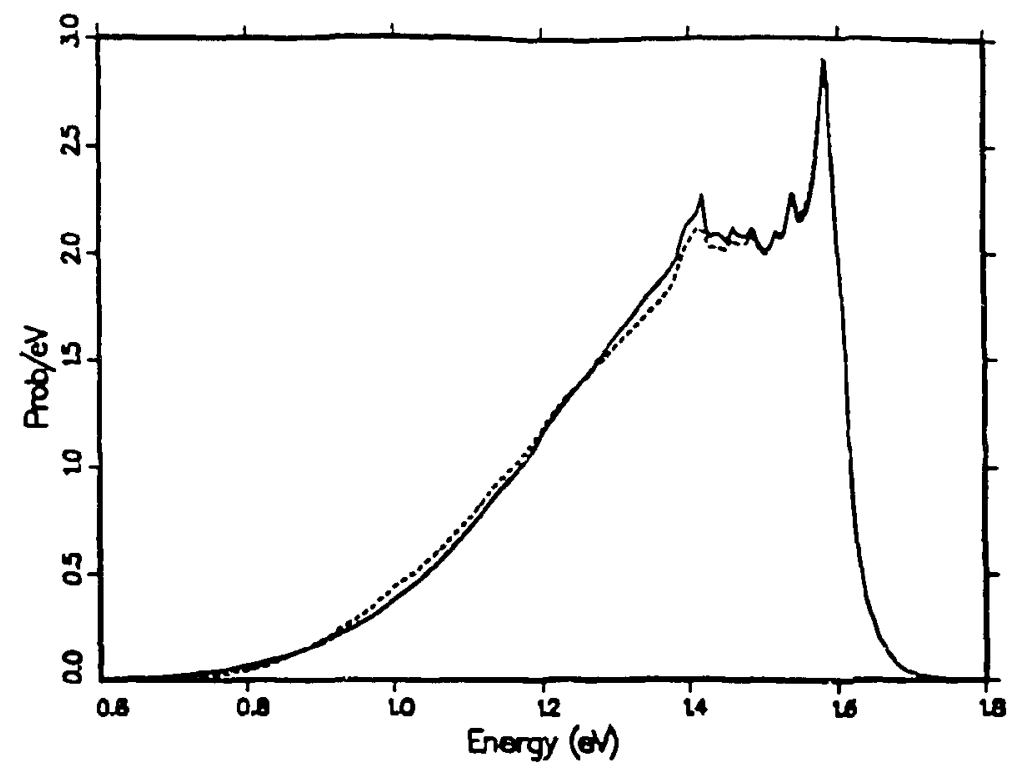

Figure 39: Expanded view of the spectrum from thermal inelastic scattering from graphite at $296 \mathrm{~K}$ with an incident energy of $1.595 \mathrm{eV}$.

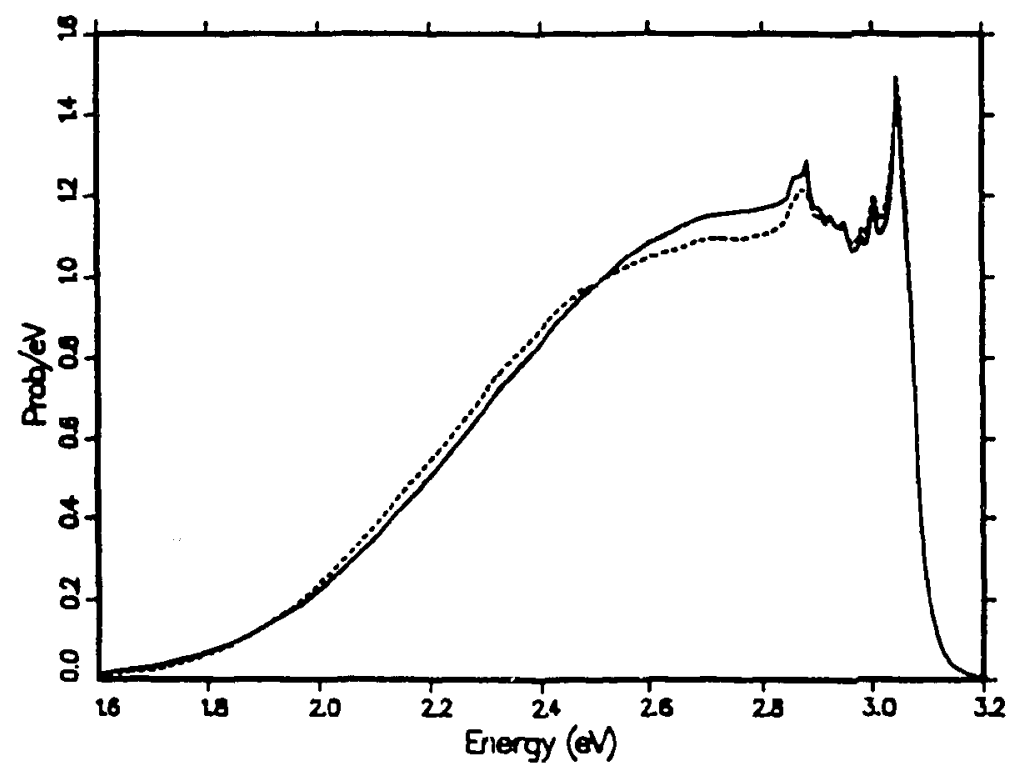

Figure 40: Expanded view of the spectrum from thermal inelastic scattering from graphite at $296 \mathrm{~K}$ with an incident energy of $3.059 \mathrm{eV}$. 


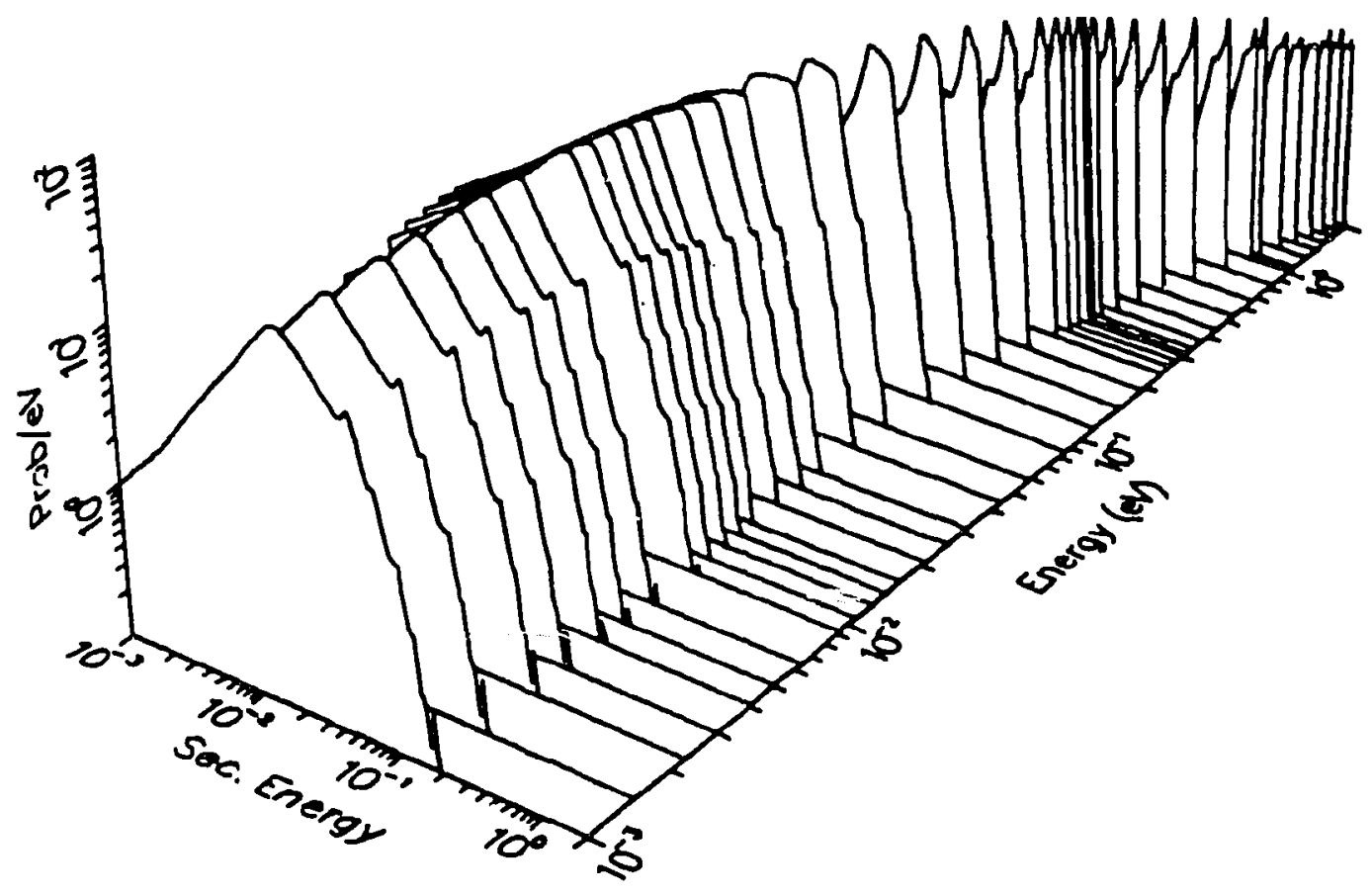

Figure 41: Perspective view of the spectra from thermal inelastic scattering on graphite at $296 \mathrm{~K}$ for energies up to $2 \mathrm{eV}$. 
Table 4: Table of Debye-Waller Integrals and Effective Temperatures for Graphite

\begin{tabular}{ccc}
\hline $\begin{array}{c}\text { Temperature } \\
\text { (deg K) }\end{array}$ & $\begin{array}{c}\text { Debye-Waller Int. } \\
\left(\mathrm{eV}^{-1}\right)\end{array}$ & $\begin{array}{c}\text { Effective Temp. } \\
\text { (deg K) }\end{array}$ \\
\hline 296 & 26.17 & 714.3 \\
400 & 32.65 & 755.5 \\
500 & 39.15 & 807.4 \\
600 & 45.81 & 869.1 \\
700 & 52.59 & 938.3 \\
800 & 87.29 & 1013. \\
1000 & 73.30 & 1175. \\
1200 & 87.29 & 1349. \\
1600 & 115.5 & 1713. \\
2000 & 143.8 & 2091. \\
\hline
\end{tabular}




\section{WATER}

The ENDF model for water assumes that the scattering law for the primary scatterer $(\mathrm{H})$ is well represented by a set of hindered rotations given as a solid-type frequency distribution, two discrete oscillators (.205 and .408 eV) to represent the molecular vibrations, and a free-gas translational mode using atomic weight 18 . The scattering from oxygen is represented using a free-gas law for mass 16. These choices are coded into the 8 th and 9 th cards on the following input deck:

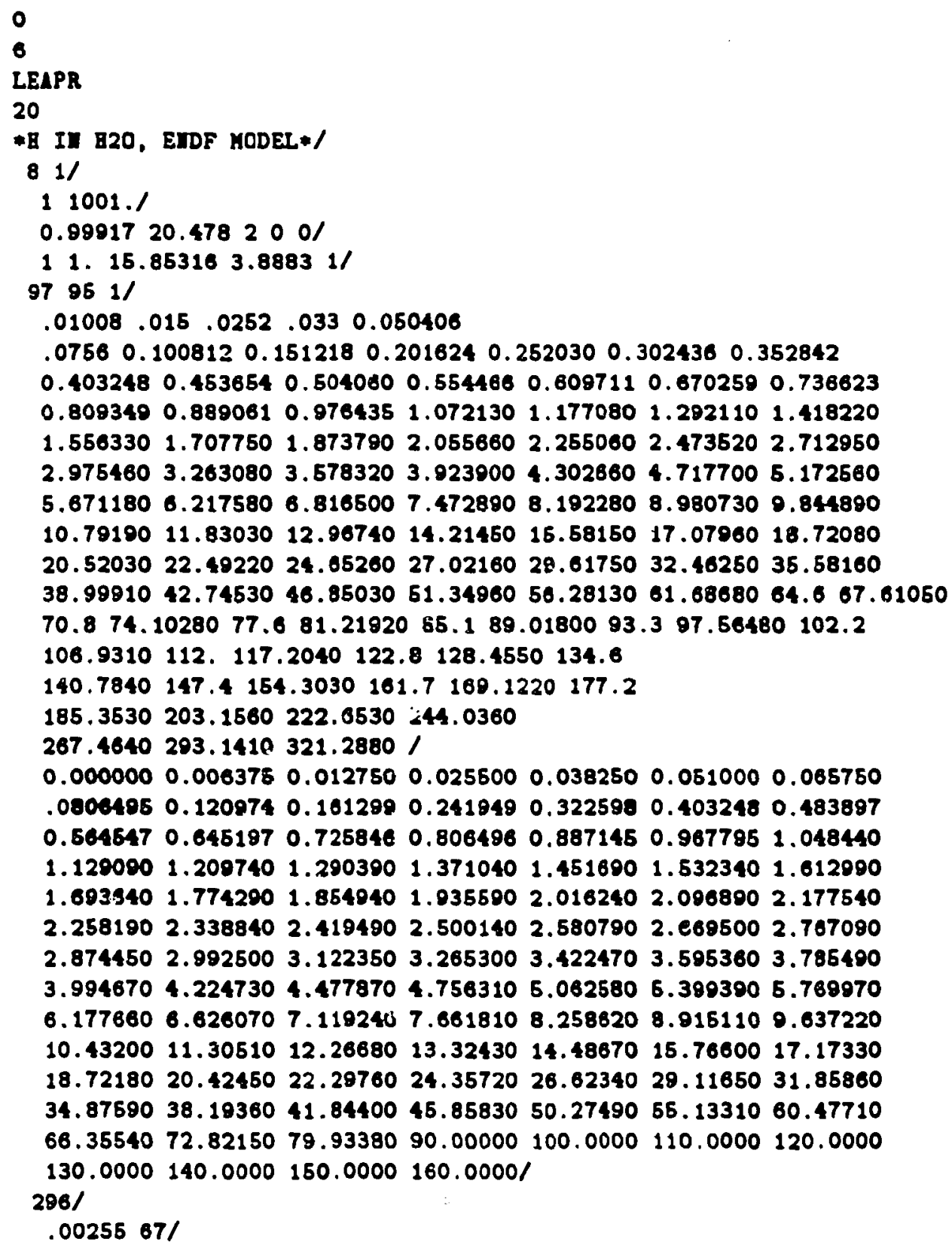




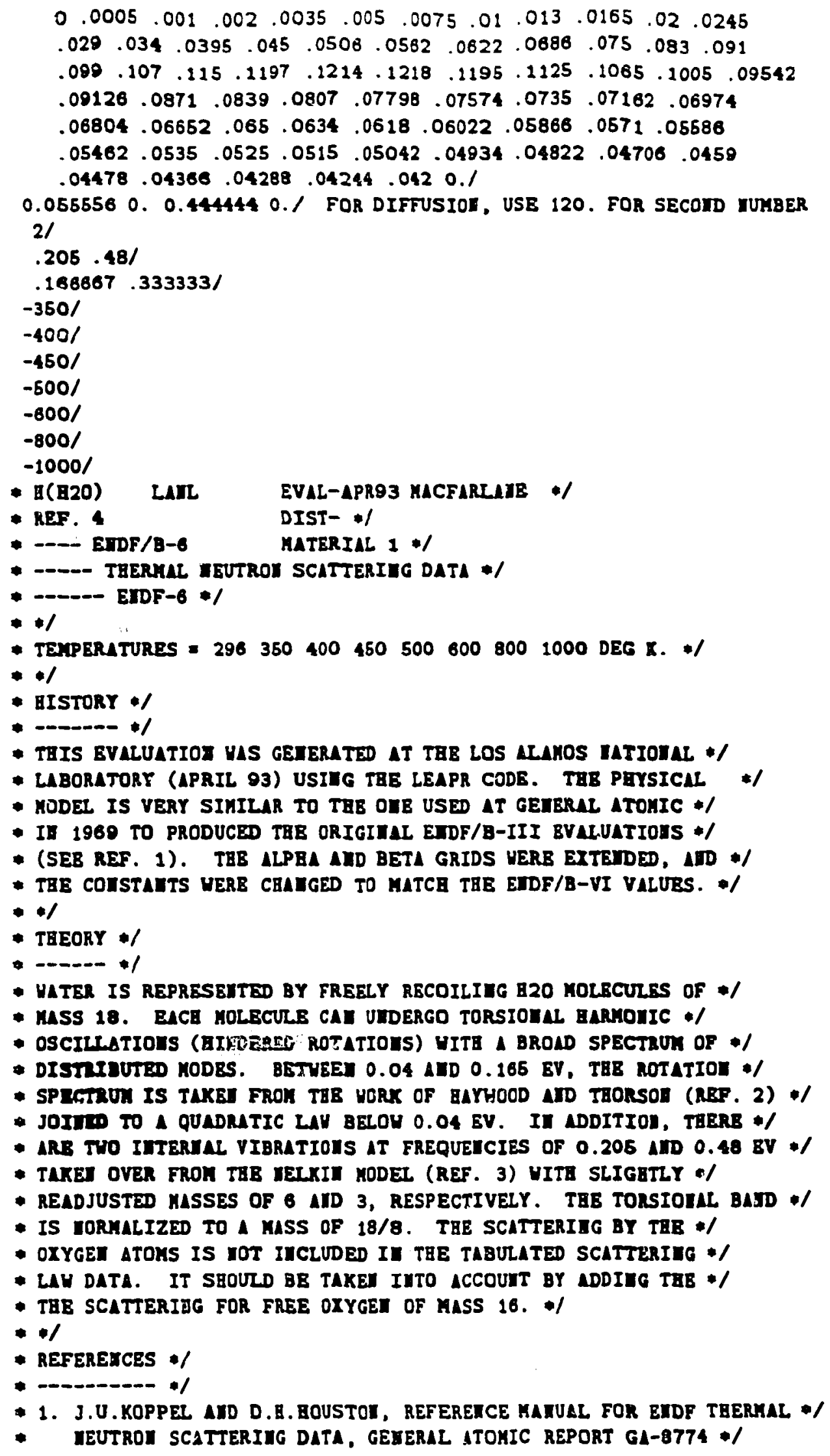


- REVISED AID REISSUED AS EXDF-269 BY TBE IATIOIAL UUCLEAR */

- data ceiter, juzy 1978. *

- 2. B.C. BaYUOOd AJD J.M.TBORSOI, "PROC. COTF. OI IEUTROI */

- TBERMLLIzLTIOI". BROOKBAVEI (1962). *I

* 3. M.S. TELKII, PEYS. REV. 119, $741(1960), *$

* 4. R.e. macf

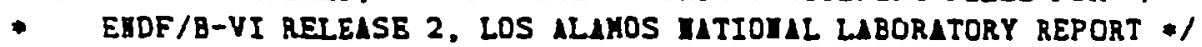

- LL-12639-NS (TO BE PUBLISEED).

$* 1$

I

STOP

These $\alpha$ and $\beta$ grids were based on the choices in the original GA evalusion. with some extensions. The input version of $\rho(\epsilon)$ couldn't use the GA values directly, because LEAPR requires a uniform grid. The figure in the GA report ${ }^{1}$ was digitized, blown up, and smoothed to get the values given in the input deck. The final frequency spectrum is shown in Figure $\mathbf{4 2 .}$

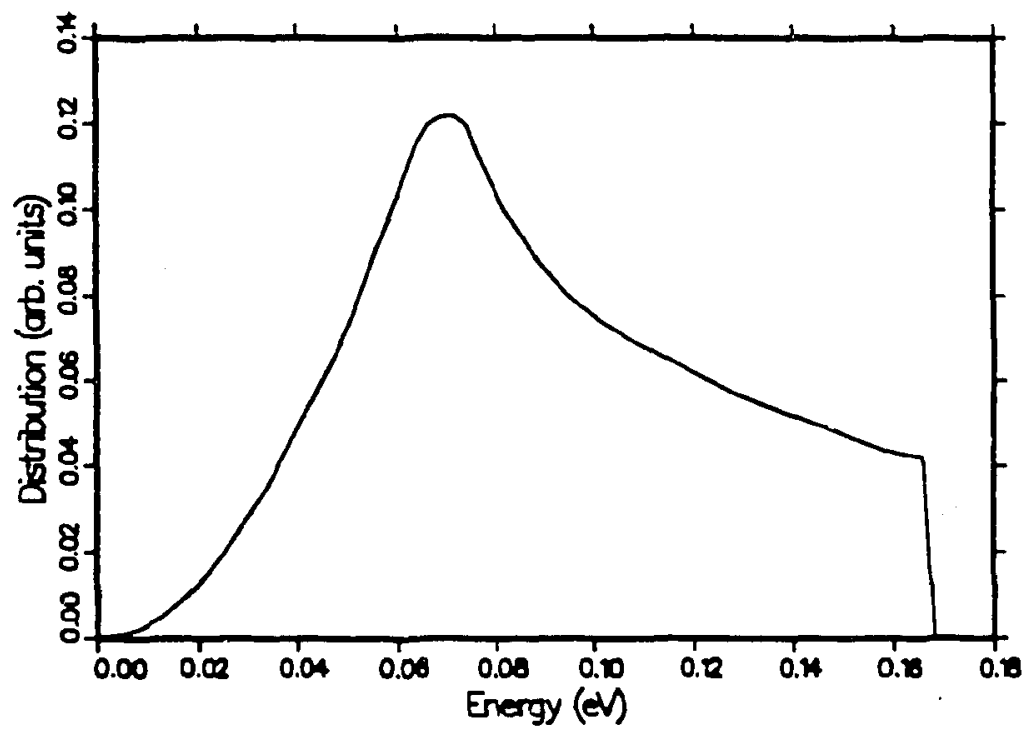

Figure 42: The frequency spectrum used for $\mathrm{H}$ in $\mathrm{H}_{2} \mathrm{O}$. It is based on the original GA model converted to a uniform grid. Note that the right-hand limit is not vertical as in the original model.

This run specifies IPRINT $=2$ to get a more detailed listing. This kind of listing includes checks for the normalization of the phonon expansion members, $\mathcal{T}_{n}$. It also prints out the values $S(\alpha, \beta), \mathcal{S}(\alpha, \beta)$, and $\mathcal{S}(\alpha,-\beta)$ for each $\beta$. Only the asymmetric $S$ for $-\beta$ is actually used and stored inside the code. The other two versions are computed just before being printed. On a short-word machine, these 
first two styles of $S$ may underflow and be printed as zero, even though the last column is nonzerc. No accuracy is actually lost, at this point.

After the results for the solid-type rotational modes have been printed out, the code starts a print for the convolution of the translational modes with the continuous modes. For each $\alpha$, the values for $S_{\text {free }}$ are printed out, followed by the results of the convolution, and the results of the normalization and sum-rule tests. These results can be examined to see if the $\beta$ grid seems to be sufficient for the problem. The problem here is that the translational peak is very sharp for small $\alpha$, and it is difficult to make the $\beta$ grid fine enough to represent it well. Some loss in the normalization and sum-rule accuracies must be accepted.

Next, the code shows similar results for the convolution of the discrete oscillators with the current scattering law. Now the problem is that new peaks appear at the $n \beta_{i}$ values and their various sums and differences. For small $\alpha$, these peaks are very sharp. A few additional $\beta$ points can be added near the peaks to improve the results, but it is usually impractical to represent them with full fidelity. Once again, some loss in the accuracy of the checks must be accepted.

Finally, a summary of the effective temperature and Debye-Waller factor is printed out, and the ENDF output file is constructed. The scattering kernel produced by running the new evaluation through THERMR is shown in Figs. 43 through 45.

The cross sections and secondary neutron distributions computed using the THERMR module of NJOY are shown in Figs. 46 through 49, and two perspective views of the secondary energy distribution are shown in Figs. 50 and 51 . The new results seem to be slightly improved over ENDF/B-VI.0 in the 1 to 3 $\mathrm{eV}$ range, although the cross section may be a little low between 3 and $4 \mathrm{eV}$.

A table of the effective temperatures and Debye-Waller integrals for $\mathrm{H}$ in $\mathrm{H}_{2} \mathrm{O}$ is given at the end of this section. 


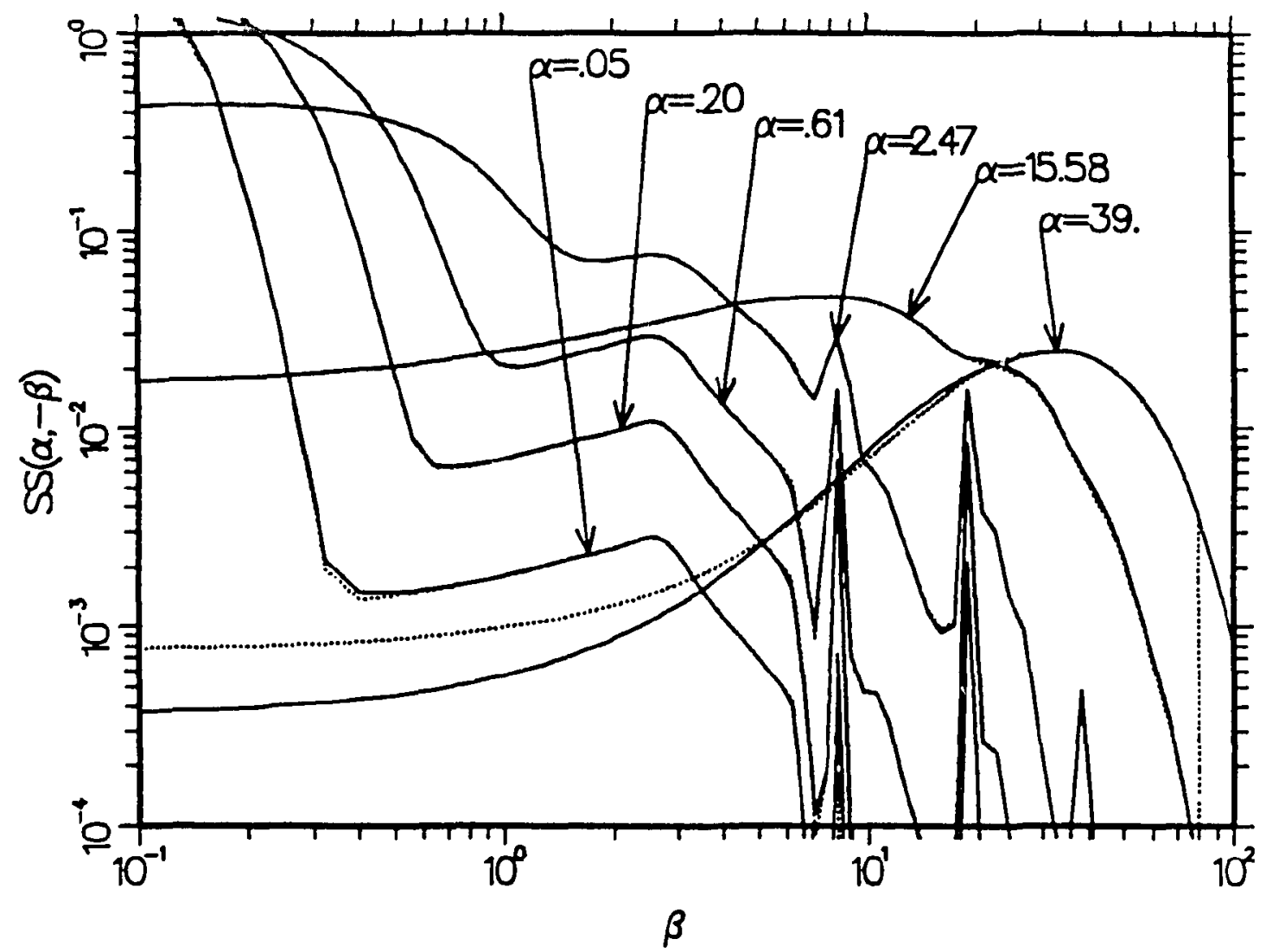

Figure 43: $\mathcal{S}(\alpha,-\beta)$ vs $\beta$ for several $\alpha$ values at a temperature of 296 $\mathrm{K}$ for $\mathrm{H}$ in $\mathrm{H}_{2} \mathrm{O}$. The solid lines are the results of this calculation, and the dashed lines are for ENDF/B-VI.0. 


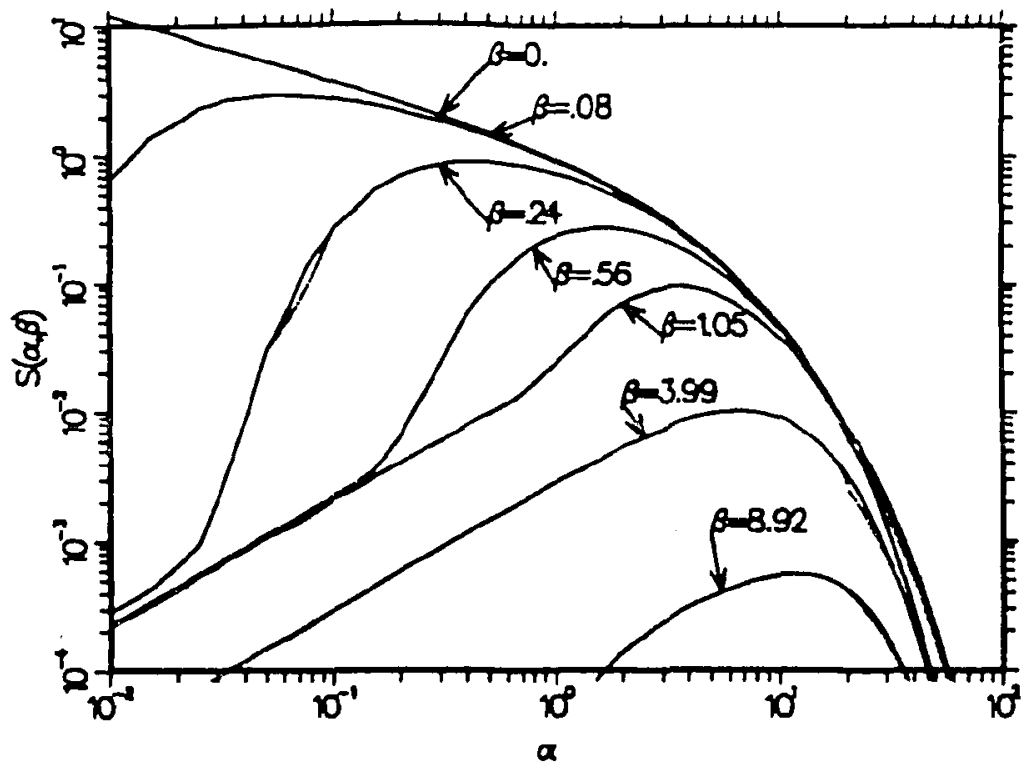

Figure 44: $S(\alpha, \beta)$ vs $\alpha$ for several $\beta$ values at a temperature of 296 $\mathrm{K}$ for $\mathrm{H}$ in $\mathrm{H}_{2} \mathrm{O}$ emphasizing the low- $\alpha$ side of the function. The solid lines are the results of this calculation, and the dashed lines are for ENDF/B-VI.0.

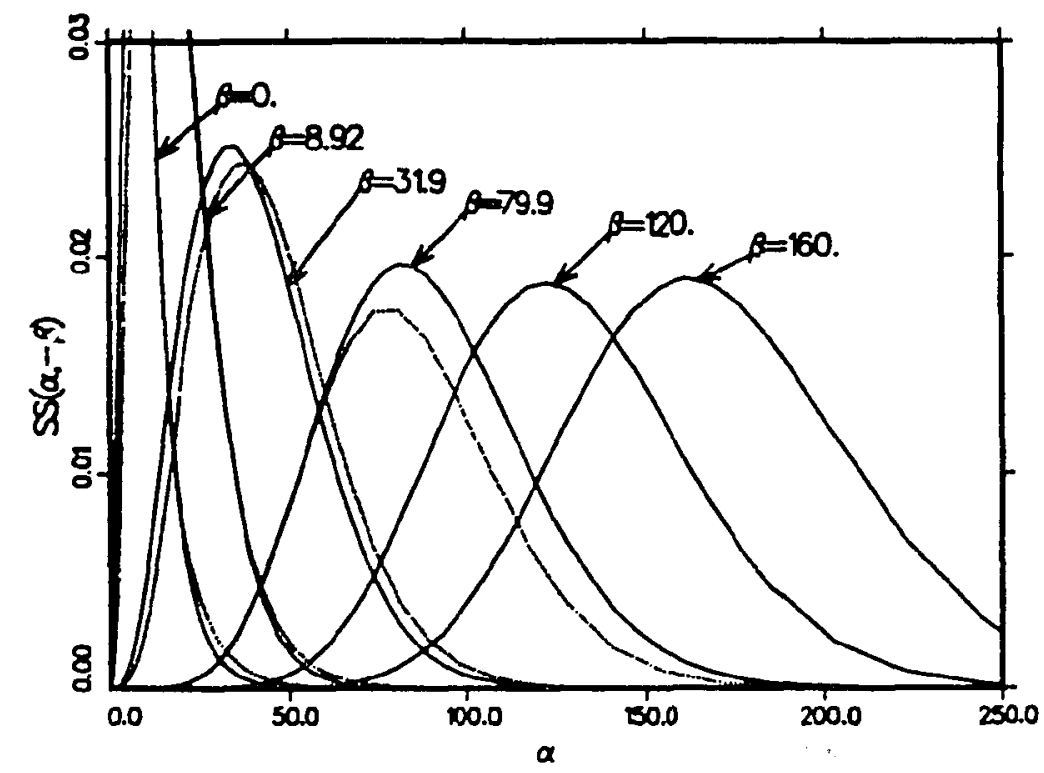

Figure 45: $\mathcal{S}(\alpha,-\beta)$ vs $\alpha$ for several $\beta$ values at a temperature of 296 $\mathrm{K}$ for $\mathrm{H}$ in $\mathrm{H}_{2} \mathrm{O}$. The solid lines are the results of this calculation, and the dashed lines are for ENDF/B-VI.0. 


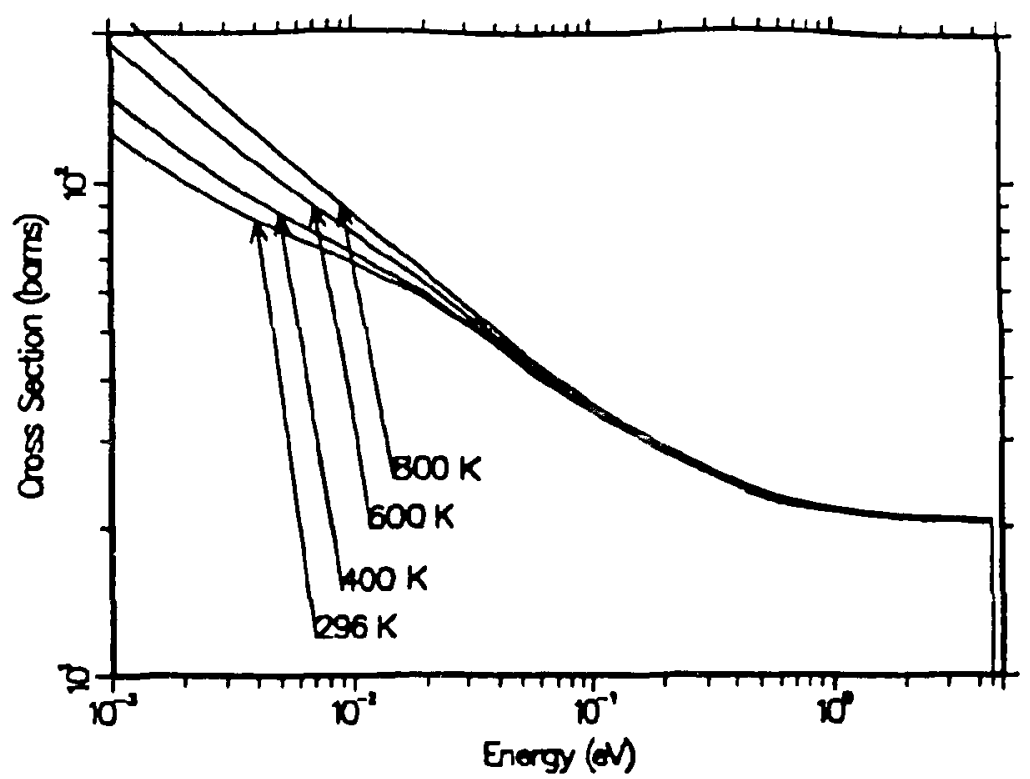

Figure 46: The inelastic cross section for $\mathrm{H}$ in $\mathrm{H}_{2} \mathrm{O}$ at four temperatures emphasizing the low-energy range. The dashed curves are for the ENDF/B-VI.0 evaluation.

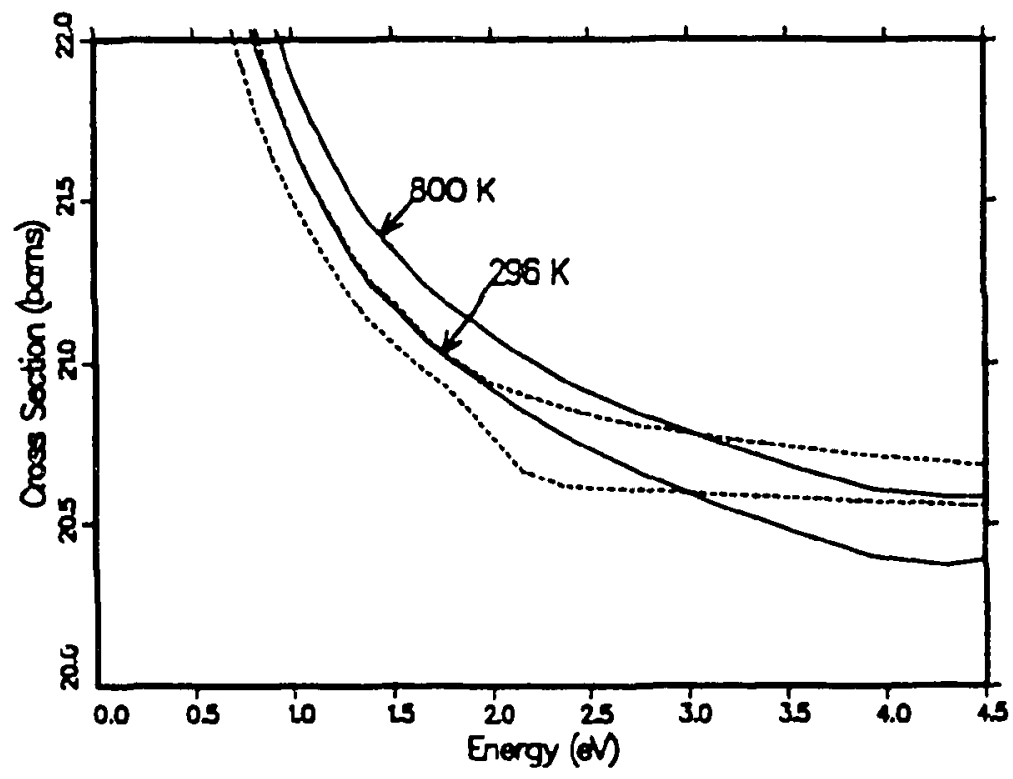

Figure 47: The inelastic cross section for $\mathrm{H}$ in $\mathrm{H}_{2} \mathrm{O}$ at temperatures of $296 \mathrm{~K}$ and $800 \mathrm{~K}$ emphasizing the high-energy range. The dashed curves are for the ENDF/B-VI.0 evaluations. 


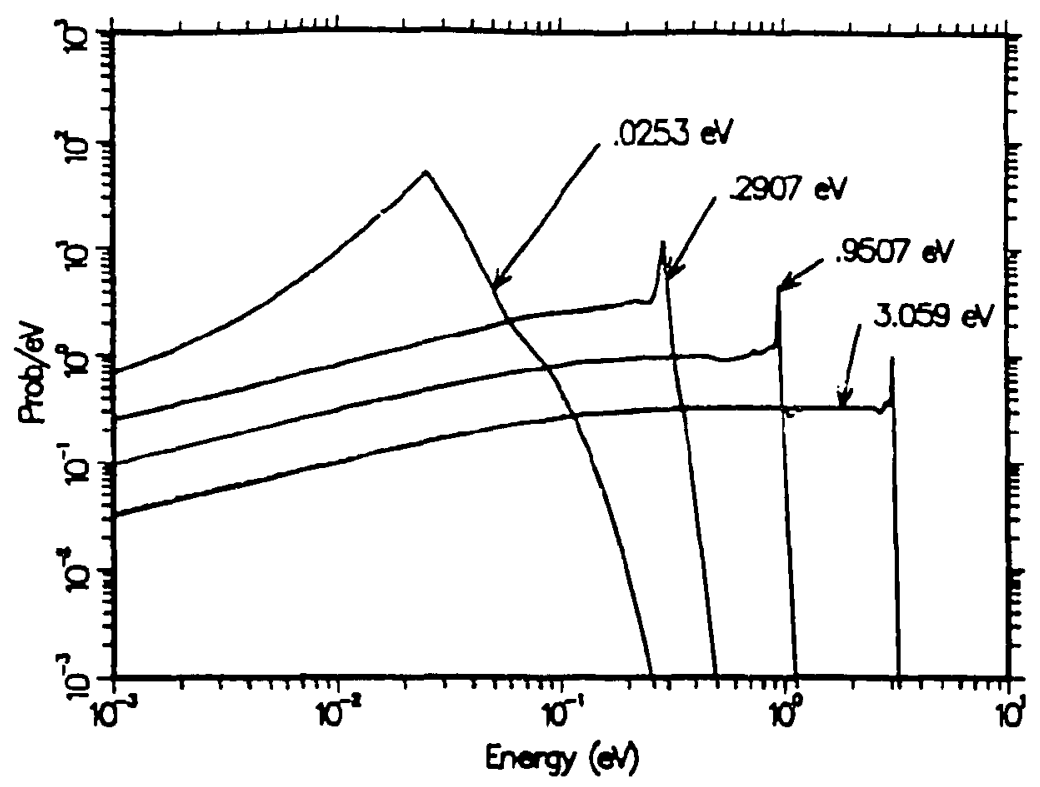

Figure 48: Secondary neutron spectra for $\mathrm{H}$ in $\mathrm{H}_{2} \mathrm{O}$ for several incident energies at a temperature of $296 \mathrm{~K}$. The dashed curves are for the ENDF/B- $\backslash$ I.0 evaluation.

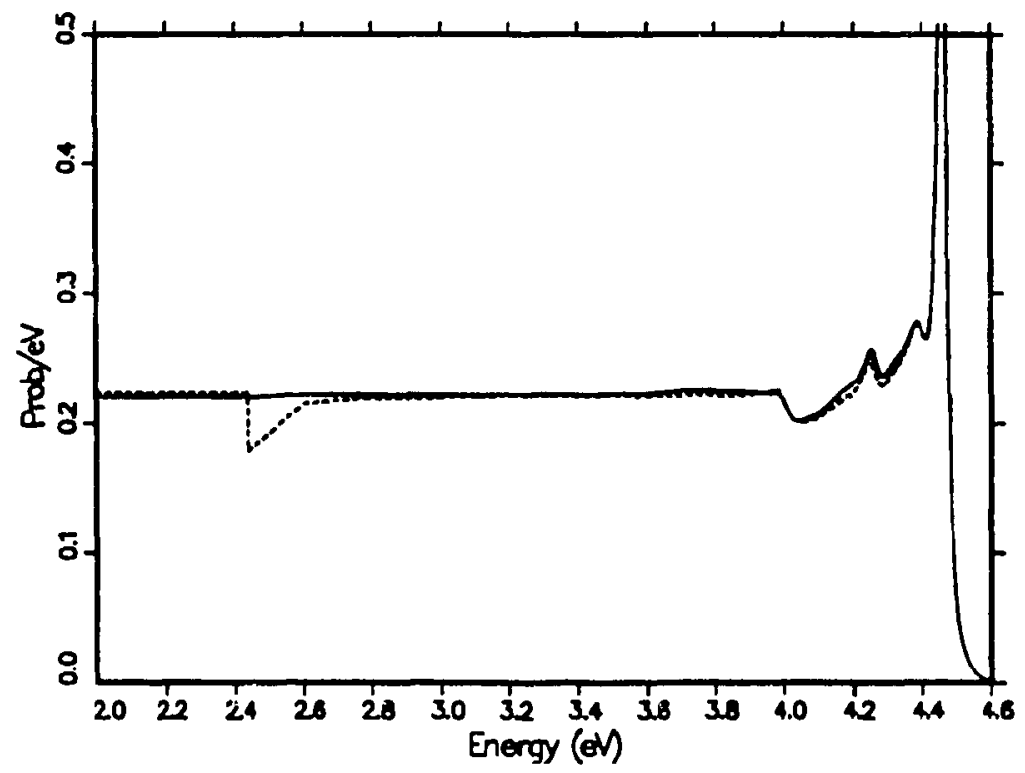

Figure 49: Expanded view of the secondary neutron spectrum at 4.46 $\mathrm{eV}$ for $\mathrm{H}$ in $\mathrm{H}_{2} \mathrm{O}$ at a temperature of $296 \mathrm{~K}$. The dashed curve is the ENDF/B.VI.0 evaluation. The notch shows where the old evaluation used the SCT approximation. 


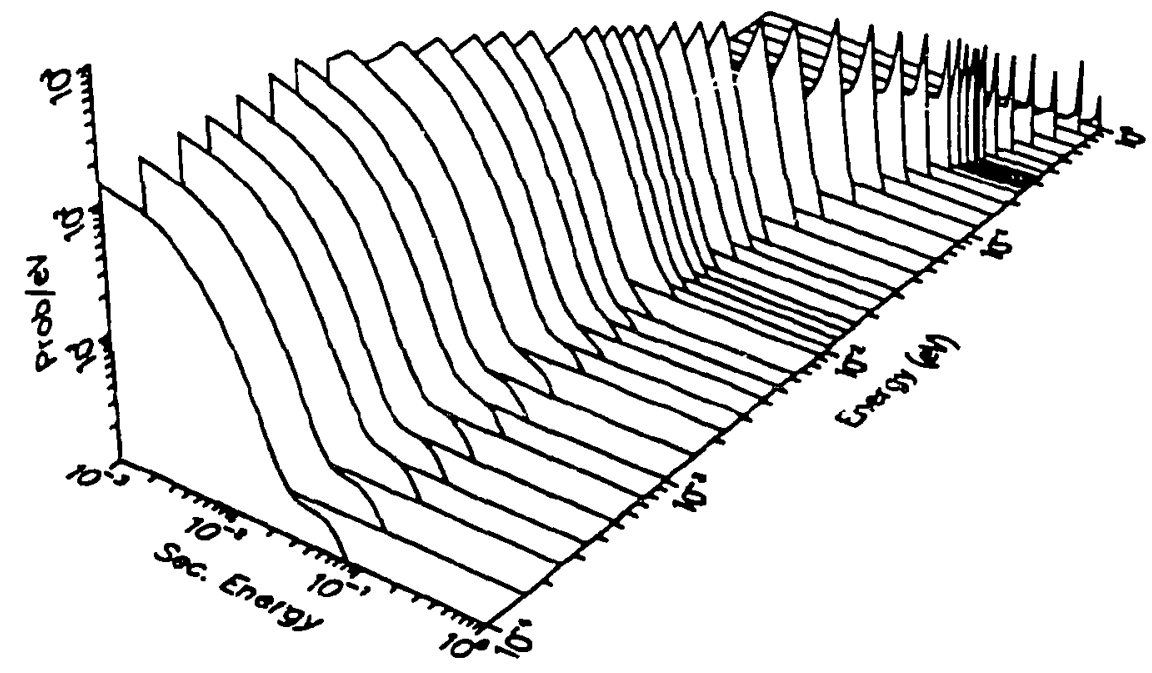

Figure 50: The isotropic component of the scattering from hydrogen bound in water for energies up to $1 \mathrm{eV}$.

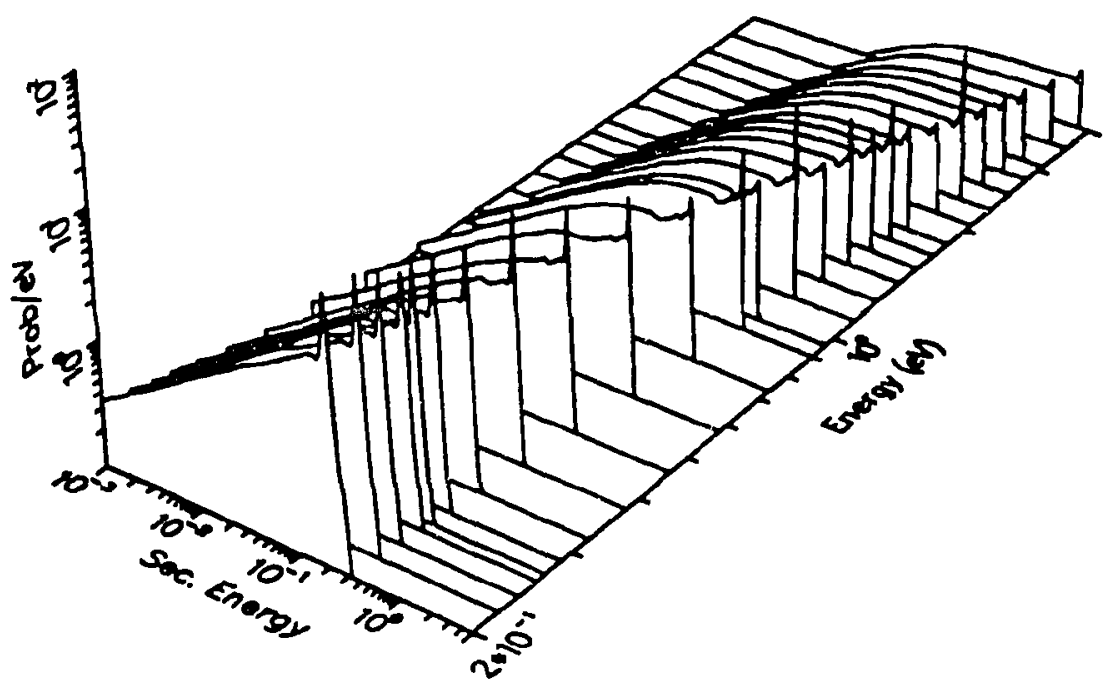

Figure 51: The isotropic component of the scattering from hydrogen bound in water for higher energies. Note that the results begin to converge to the free-gas shape except for some sharp features near $E=E^{\prime}$. The variation in the size of the pseudo-elastic peak resulting from the free translation modes is an artifact of the plotting program. 
Table 5: Table of Debye-Waller Integrals and Effective Temperatures for $\mathrm{H}$ in $\mathrm{H}_{2} \mathrm{O}$

\begin{tabular}{ccc}
\hline $\begin{array}{c}\text { Temıperature } \\
\text { (deg K) }\end{array}$ & $\begin{array}{c}\text { Debye-Waller Int. } \\
\left(\mathrm{eV}^{-1}\right)\end{array}$ & $\begin{array}{c}\text { Effective Temp. } \\
(\text { deg K })\end{array}$ \\
\hline 296 & 10.73 & 1368. \\
350 & 11.68 & 1381. \\
400 & 12.62 & 1395. \\
450 & 13.59 & 1410. \\
500 & 14.59 & 1428. \\
600 & 16.67 & 1466. \\
800 & 21.00 & 1556. \\
1000 & 25.48 & 1660. \\
\hline
\end{tabular}




\section{ZIRCONIUM HYDRIDE}

Zirconium hydride, $\mathrm{ZrH}_{x}$, has variable stoichiometry, with $x$ near 2 . Therefore, it is necessary to treat it as two separate materials, namely, $\mathrm{H}$ bound in $\mathrm{ZrH}_{x}$, and $\mathrm{Zr}$ bound in $\mathrm{ZrH}_{x}$. The calculation for $\mathrm{H}$ bound in $\mathrm{ZrH}_{x}$ follows the GA model, except the $\alpha$ and $\beta$ grids are extended, and the various constants were updated to be consistent with ENDF/B-VI. The frequency spectrum was based on a central-force lattice dynamics model. ${ }^{\text {i9 }}$ This model approximated the slightly tetragonal lattice structure of $\mathrm{ZrH}_{2}$ by a face-centered cubic lattice. Four force constants- $\mu, \gamma, \nu$, and $\delta$-were introduced describing respectively the interaction of a zirconium atom with its nearest neighbors $(8 \mathrm{H}$ atoms), the next nearest neighbors ( $12 \mathrm{Zr}$ atoms), and the interaction of a hydrogen atom with its next nearest neighbors (6 $\mathrm{H}$ atoms) and its third nearest neighbors (12 $\mathrm{H}$ atoms). The final values of the force constants were obtained by fitting both specific heat and neutron data. The position of the optical peak-observed by neutron scattering techniques to be centered roughly around $0.14 \mathrm{eV}$-determines the constant $\mu$, while the overall width and shape of this peak determine $\nu$ and $\delta$, respectively. Existing neutron data were not sufficiently precise to confirm the structure predicted in the optical peak by the central force model. Specific heat data were used to determine the force constant $\gamma$, which primarily determines the upper limit on the phonon energies associated with the acoustic modes. Eigenvalues and eigenvectors of the dynamical matrix corresponding to this model were calculated, and a phonon frequency spectrum was obtained by root sampling techniques. The following block of computer code shows the actual input file used for the $\mathrm{H}(\mathrm{ZrH})$ run.

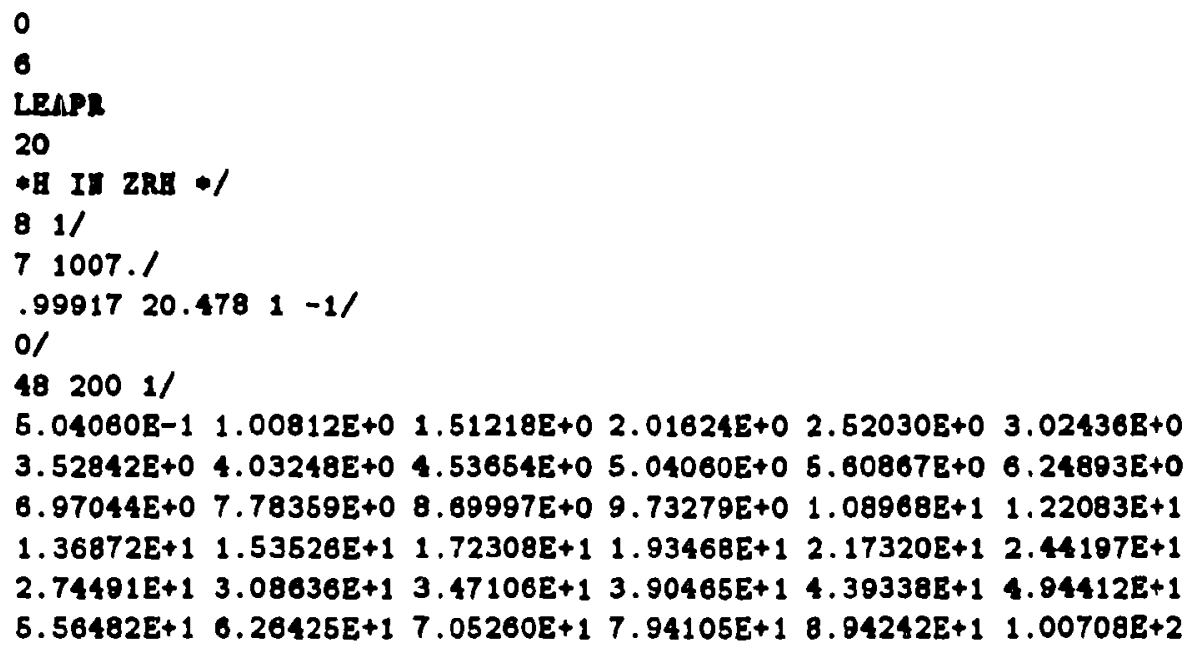


$1.13423 E+21.27759 E+21.43909 E+21.62126 E+2$

170. 180. 190. 200. 210. 220. 230. 240./

$0.00000 \mathrm{E}+0 \quad 7.90678 \mathrm{E}-2 \quad 1.58134 \mathrm{E}-1 \quad 2.37200 \mathrm{E}-1 \quad 3.16277 \mathrm{E}-1 \quad 3.95344 \mathrm{E}-1$

4.74411E-1 5.13939E-1 5.53478E-1 5.93016E-1 6.32545E-1 B.72083E-1

$7.11611 \mathrm{E}-1$ 7.51150E-1 7.90678E-1 8.30217E-1 8.68755E-1 9.48822E-1

$1.02788 E+0 \quad 1.10691 E+0 \quad 1.18605 E+0 \quad 1.26509 E+0 \quad 1.3412 E+0 \quad 1.46278 E+0$

$1.58134 E+0 \quad 1.69998 E+0 \quad 1.81855 E+0 \quad 1.93720 E+0 \quad 2.05676 E+0 \quad 3.17441 E+0$

$2.29297 E+0 \quad 2.41162 E+0 \quad 2.53018 E+0 \quad 2.88594 E+0 \quad 3.20229 E+0 \quad 3.51854 E+0$

$3.87430 E+0 \quad 4.15103 E+0 \quad 4.46738 E+0 \& .58594 E+0 \quad 4.66507 E+0 \& .74411 E+0$

$4.82314 E+0 \quad 4.90218 E+0 \quad 4.98132 E+0 \quad 5.06036 E+0 \quad 5.13039 E+0 \quad 5.21853 E+0$ E. $29757 E+0 \quad 5.33708 E+0 \quad 5.37660 E+0 \quad 5.41612 E+0 \quad 5.45674 E+0 \quad 5.53478 E+0$ $5.613818+0 \quad 5.69295 E+0 \quad 5.77199 E+0 \quad 5.81150 \mathrm{~B}+0 \quad 5.86102 E+0 \quad 5.89054 E+0$ $5.93016 E+0 \quad 5.96968 E+0 \quad 6.00920 E+0 \quad 6.08823 E+0 \quad 6.16727 E+0 \quad 6.24641 E+0$ $6.32545 \mathrm{E}+0 \quad 6.91842 \mathrm{E}+0 \quad 7.51150 \mathrm{E}+0 \quad 8.10447 \mathrm{E}+0 \quad 0.09283 \mathrm{~B}+0 \quad 0.40908 \mathrm{~B}+0$ $0.72543 E+0 \quad 1.004178+1 \quad 1.03584 E+1 \quad 1.06740 B+1 \quad 1.07536 B+1 \quad 1.08322 B+1$ $1.09119 \mathrm{E}+1 \quad 1.10601 \mathrm{~B}+1 \quad 1.12274 \mathrm{E}+1 \quad 1.13867 \mathrm{~B}+1 \quad 1.1540 \mathrm{~B}+1 \quad 1.16226 \mathrm{~B}+1$ $1.17023 B+1 \quad 1.17809 E+1 \quad 1.18605 E+1 \quad 1.19392 E+1 \quad 1.20188 B+1 \quad 1.23343 B+1$ $1.26509 \mathrm{~B}+1 \quad 1.30461 \mathrm{~B}+1 \quad 1.36388 \mathrm{E}+1 \quad 1.42326 \mathrm{~B}+1 \quad 1.48254 \mathrm{~B}+1 \quad 1.54182 \mathrm{~B}+1$ $1.67740 \mathrm{~B}+1 \quad 1.60110 \mathrm{E}+1 \quad 1.62489 \mathrm{E}+1 \quad 1.64858 \mathrm{~B}+1 \quad 1.67237 \mathrm{~B}+1 \quad 1.69606 \mathrm{~B}+1$ $1.71075 \mathrm{E}+1 \quad 1.74344 \mathrm{~B}+1 \quad 1.76713 \mathrm{E}+1 \quad 1.79092 \mathrm{~B}+1 \quad 1.81855 \mathrm{~B}+1 \quad 1.86020 \mathrm{~B}+1$ $1.80798 E+1 \quad 1.97672 \mathrm{E}+1 \quad 2.05576 \mathrm{E}+12.09527 \mathrm{~B}+1 \quad 2.13470 \mathrm{~B}+12.1744 \mathrm{~B}+1$ $2.21393 B+12.25345 B+12.29287 E+12.33248 B+12.37200 B+12.46114 B+1$ $2.53018 B+12.56970 \mathrm{~B}+12.80921 \mathrm{E}+12.64883 \mathrm{~B}+12.68835 \mathrm{~B}+12.72787 \mathrm{E}+1$ 2.76739B+1 2.80091E+1 2.84642E+1 2.88594B+1 2.92556B+1 2.96508B+1 $3.02436 \mathrm{~B}+1 \quad 3.08363 \mathrm{~B}+1 \quad 3.12316 \mathrm{E}+1 \quad 3.16277 \mathrm{E}+1 \quad 3.20220 \mathrm{~B}+1 \quad 3.24181 \mathrm{~B}+1$ $3.28133 B+13.32085 E+1 \quad 3.36036 B+13.39988 B+1 \quad 3.43960 B+1 \quad 3.47902 B+1$ $3.55806 \mathrm{~B}+1 \quad 3.63709 \mathrm{~B}+1 \quad 3.71623 \mathrm{E}+13.79527 \mathrm{E}+1 \quad 3.87430 \mathrm{~B}+1 \quad 3.96344 \mathrm{~B}+1$

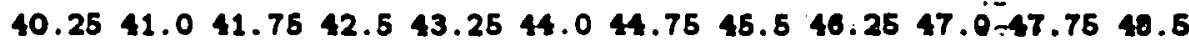
$49.25 \quad 50.0 \quad 51.75 \quad 52.5 \quad 53.25 \quad 54.0 \quad 54.75 \quad 55.5 \quad 56.25 \quad 57.0 \quad 57.75 \quad 58.5$ $59.2560 .0 \quad 60.75 \quad 61.5 \quad 62.2563 .0 \quad 63.75 \quad 64.5 \quad 65.25 \quad 66.0 \quad 66.75 \quad 67.5$ $68.2569 .0 \quad 69.75 \quad 70.5 \quad 71.2572 .072 .75 \quad 73.5 \quad 74.2575 .0 \quad 75.75 \quad 76.5$ $77.2578 .0 /$

296/

$.001161 /$

0. $.000875 .0036 \quad .008 \quad 016 \quad 0235 \quad .0340$

$\begin{array}{lllll}.046 & .061 & .078 & .084 .116 \quad .144\end{array}$

$\begin{array}{lllll}.1606 & .1960 .2606 & .3479 & .3559 & .3500\end{array}$

$\begin{array}{lllllll}.3322 & .3328 & .2011 & .1617 & .1431 & .1248\end{array}$

$\begin{array}{llllll}.09738 .06067 & .1221 & .1495 & .07219 & .01443\end{array}$

.00018220 .04992 .0103 .560

$4.790 \quad 5.995 \quad 7.250 \quad 8.550 \quad 9.640 \quad 11.91$

$\begin{array}{lllllll}13.52 & 16.04 & 10.79 & 26.10 & 29.39 & 30.82\end{array}$

32.2131 .7533 .1435 .6533 .3436 .27

$\begin{array}{lllllll}30.18 & 38.75 & 30.48 & 28.89 & 23.29 & 25.18\end{array}$

$\begin{array}{lllllll}26.59 & 27.86 & 27.89 & 29.44 & 25.86 & 23.33\end{array}$

$\begin{array}{llllll}24.66 & 27.51 \quad 37.94 & 60.77 & 26.68 & 18.54\end{array}$

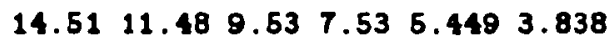

8.4970 .1

0.0 .1 .0 .1

$0 /$

$-400.1$

$-500.1$

$-600.1$

$-700 . /$

$-800 . /$ 
$-1000 . /$

$-1200.1$

* B(2R日) LARL EVAL-IPR73 MACFARLAIE*/

- REF. 3 DIST-*/

*-- EIDF/B-6 MLTERIAL 7*/

* - - TEERHal IEUTROI SCATTERIJg DATA*/

- - - ENDF-6*/

* *

- TEhPERAtures $=29840050060070080010001200$ DEG K.*/

* $/$

- BISTORY*/

* - -

* This bValuatiog has produced at los alamos Ig 1903 USIggó

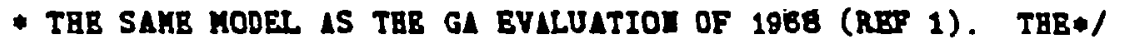

- leapr module of hjot uas uszo for tab calculation, and*/

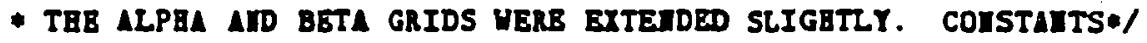

- THAT HATCE TAB EJDF/B-VI VALUES UERB USED.*/

$* 1$

- TBEORY*/

$+-2---\infty /$

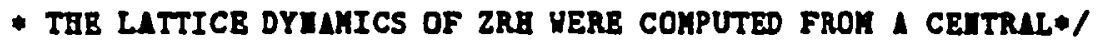

- Force hodel. the sligatzy tetalgotal latticb of zRE_2 uas*/

* APPROXIMATED BY A FACB-CEITERED-CUBIC LATTICB. FOUR FORCE\#/

* COISTAUTS (MU, GAMA, IU, AID DELTA) HERB IITRODUCED DESCRIBIIG*/

* RESPECTIVELY SEE IUTERACTIOI OF. a zIRCOIIUR ATORS UITE ITS*/

* mearest meighbors ( 8 a ltons) lid its mext mearest meighbors*/

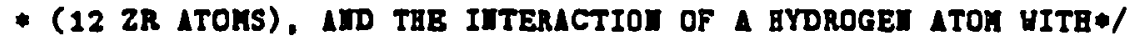

* its yext hearest meiggbors (6 a itons) add its third nearest*/

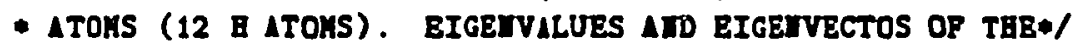

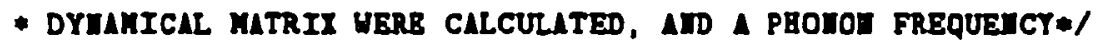

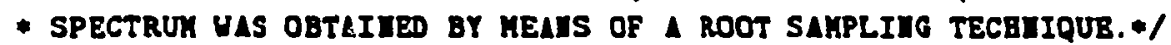

- VEIGETED FREQUEJCY SPECTRA FOR BYDROGEJ II ZRA UERE TEEI*/.

- OBTAIEED BT APPROPRIATB USB OF THB DYMAHICAL MATRIX*/

* EIGEHVECTORS (REF. 2).*/

* 1

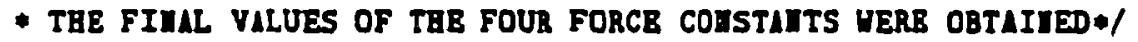

* by fITTIJg both SPECIFIC helt atd meUtroI data. TEE*/

- positida of al optical peak observed by IEUTROI scatTERIIG*/

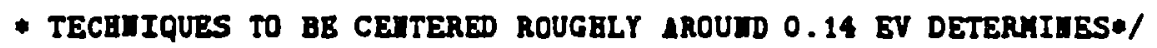

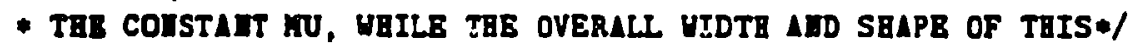

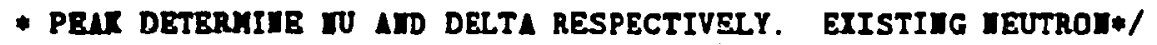

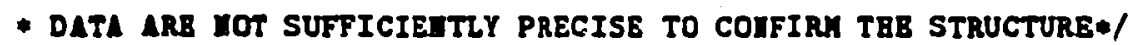

- pREdcted il teg optical peax gy teB ceItRal forcB hodel. */

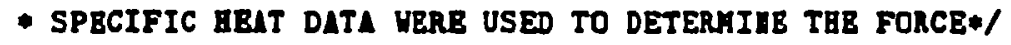

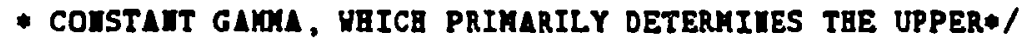

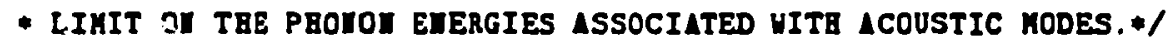

$*$ *

- REFEREjCES*/

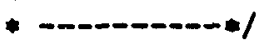

- 1. J.U.KOPPEL AYD D.8. BOUSTOM, REFEREMCE MaTUAL FOR EUDF TBERHAL*/

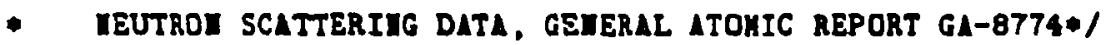

- REVISED ATD REISSUED IS ETDF-269 BY TAE matiomaL IUCLEaR*/

* data center, july 1978.*/

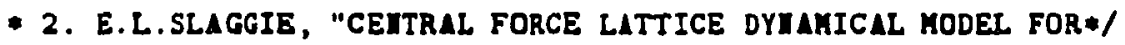

* ZIRCOIIUK BYDRIDE", GL-8132 (1967).*/ 
* 3. R.e.macfarlahe. neu theryal veutrou scatteridg files for */

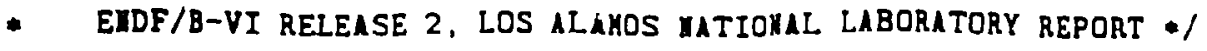

* LA-12639-KS (TO BE PUBLISBED). */

$*$ *

1

STOP

The acoustic modes and optical modes for $\mathrm{H}$ in $\mathrm{ZrH}$ are shown in Figures 52 and 53 , respectively. The strong peak near $0.14 \mathrm{eV}$ is basically an "Einstein oscillator" (they are discussed in the references on thermal scattering theory), and it leads to strong oscillations in both the scattering function and the cross sections. As a result, a very detailed $\beta$ grid is required. The new model used the GA grid below $\beta=40$ ( $1 \mathrm{eV}$ ), but it provided an extension up to $\beta=78$. This extension allows for energy transfers of almost $2 \mathrm{eV}$ at $296 \mathrm{~K}$. The result is shown in Figure 54 for low $\beta$ and Figure 55 for high $\beta$. The function $\mathcal{S}(\alpha,-\beta)$, that is, script-S for downscatter, is plotted. First, note the prominent oscillations. They extend to very high $\beta$ values when $\alpha$ is large. Second, note that the new and old evaluations agree fairly well, except for the dropouts for low values of script-S characteristic of the old evaluation (dashed curves).

To evaluate the importance of the extended $\beta$ range and the effects of removing the dropouts, the new evaluation was run through the THERMR module of NJOY to obtain integrated cross sections and $P_{0}$ scattering spectra. Figure 56 shows the inelastic part of the integrated cross section versus incident neutron energy for several temperatures. The new evaluation is almost identical to the old, except for some small differences at high energies. Figure 57 shows this high energy range in more detail at the limiting temperatures. Note that the two models begin to deviate at $1 \mathrm{eV}$ for $296 \mathrm{~K}$; this is the point where the extended $\beta$ grid begins to have an effect. Figure 58 shows the incoherent elastic part of the cross section.

The effects of the dropouts are more evident in Figures 59 and 60. Although the differences at small secondary energies are between small values of the scattering cross section, their importance may be amplified in the presence of $1 / v$ absorption. The effects of the extended $\beta$ range are very clear in Figure 61 . The low energy shape comes from the SCT approximation. The old evaluation used the approximation for all energy transfer greater than $1 \mathrm{eV}$, but the new evaluation allows transfers as large as $2 \mathrm{eV}$. The difference between these two shapes has a modest effect on the integrated cross section, as shown in Figure 56. The oscillations caused by the Einstein oscillator are dramatic. 


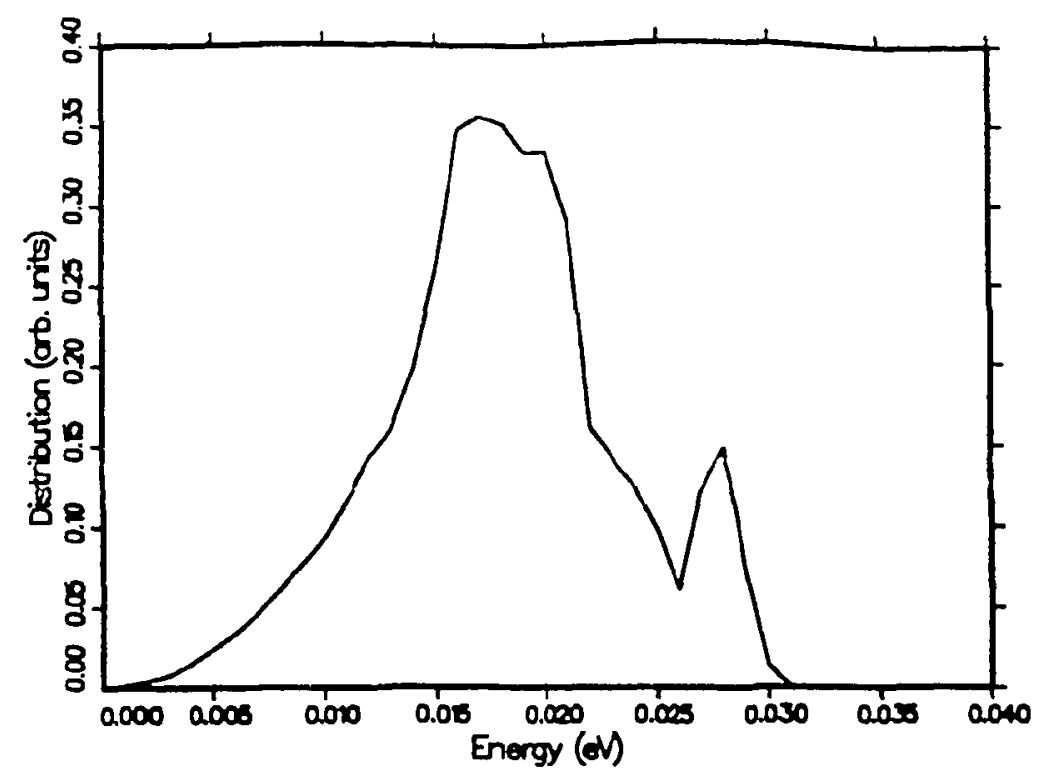

Figure 52: The frequency spectrum used for $\mathrm{H}$ in $2 \mathrm{rH}$ showing the acoustic modes.

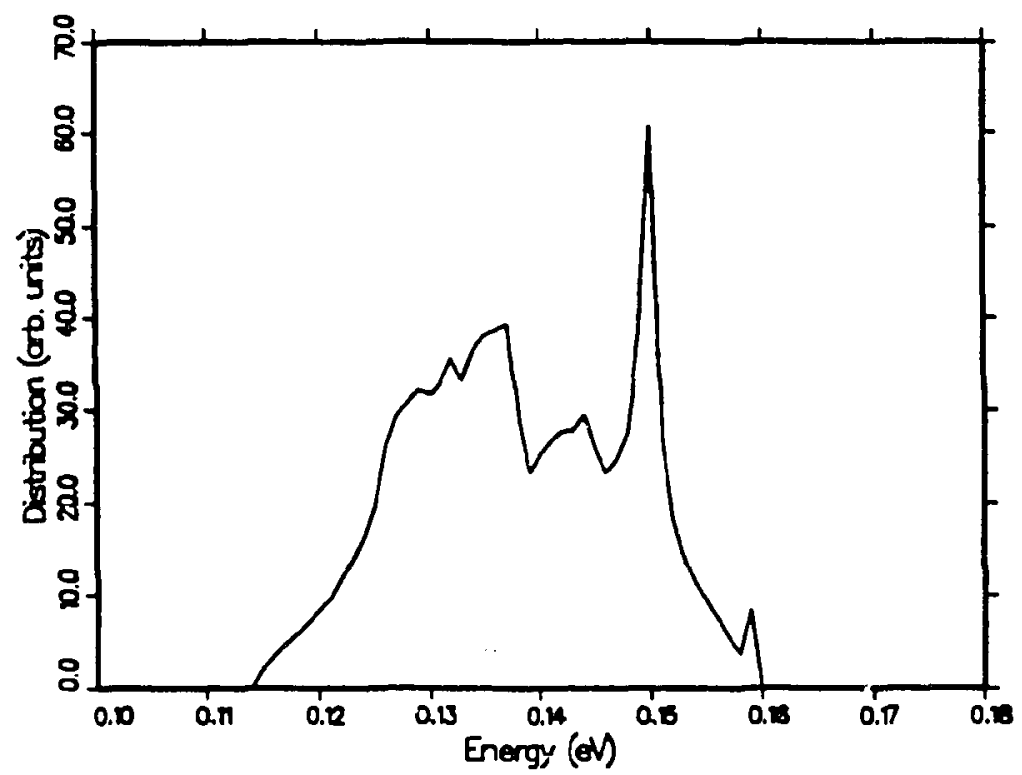

Figure 53: The frequency spectrum used for $\mathrm{H}$ in $\mathrm{ZrH}$ showing the optic modes. The function is zero between the top of the acoustic modes and the bottom of the optic modes. 


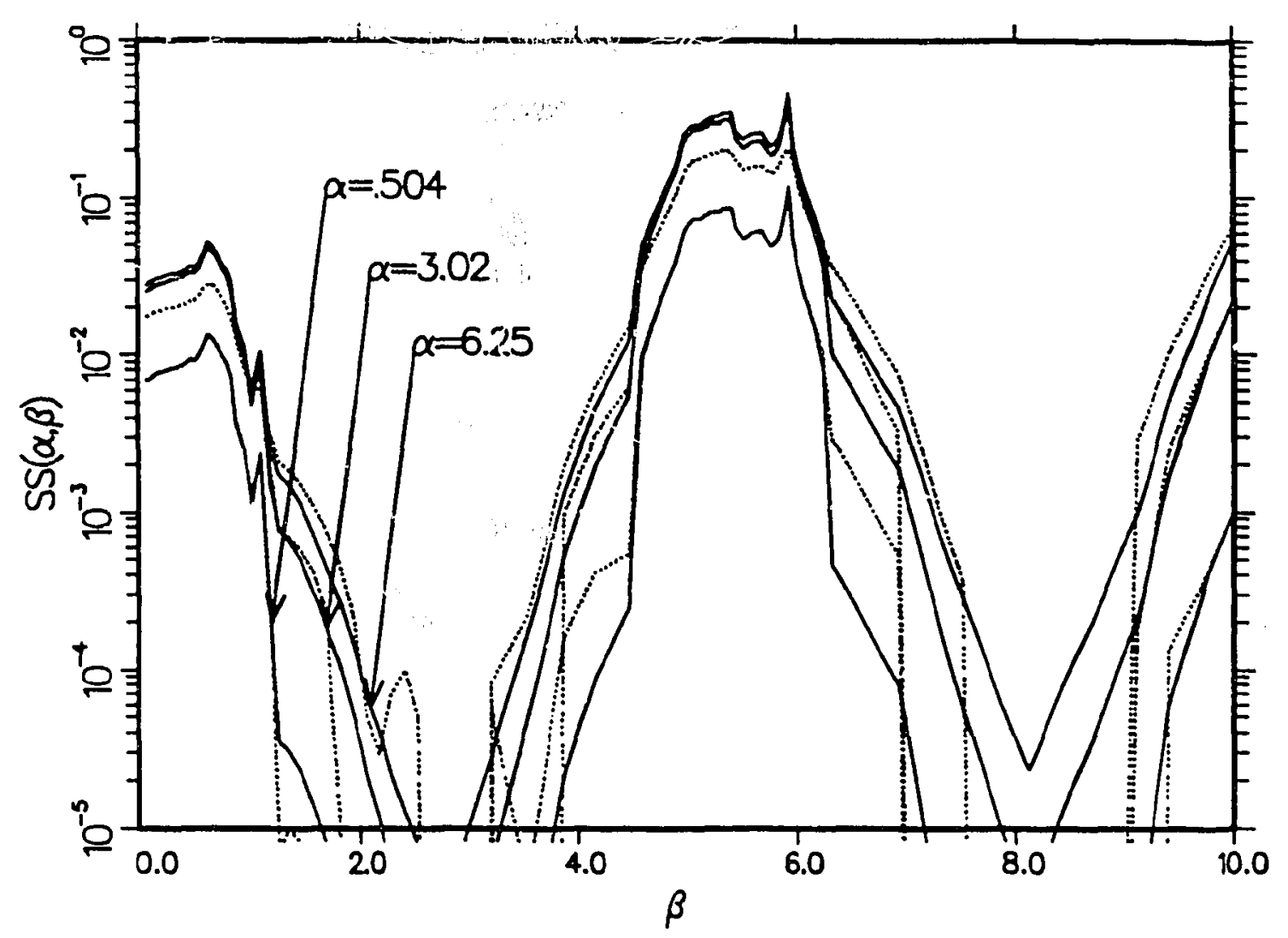

Figure 54: $\mathcal{S}(\alpha,-\beta)$ vs $\beta$ for several $\alpha$ values at a temperature of 296 $\mathrm{K}$ showing the low- $\beta$ side of the function. The solid lines are the results of this calculation, and the dashed lines are for ENDF/B-VI.0. 


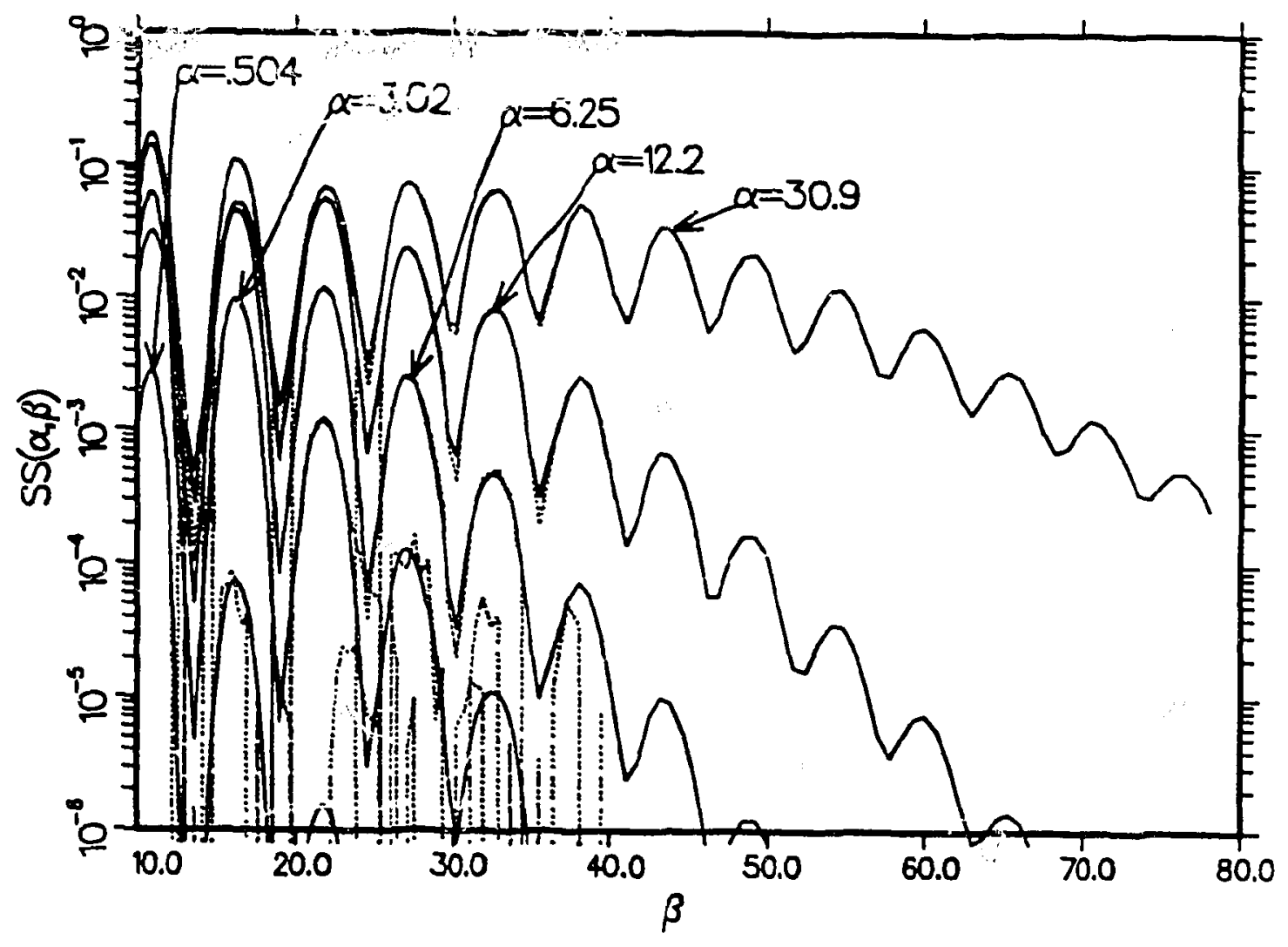

Figure 55: $\mathcal{S}(\alpha,-\beta)$ vs $\beta$ for several $\alpha$ values at a temperature of $296 \mathrm{~K}$ showing the high- $\beta$ side of the function. The solid lines are the results of this calculation, and the dashed lines are for ENDF/B.VI.O. 


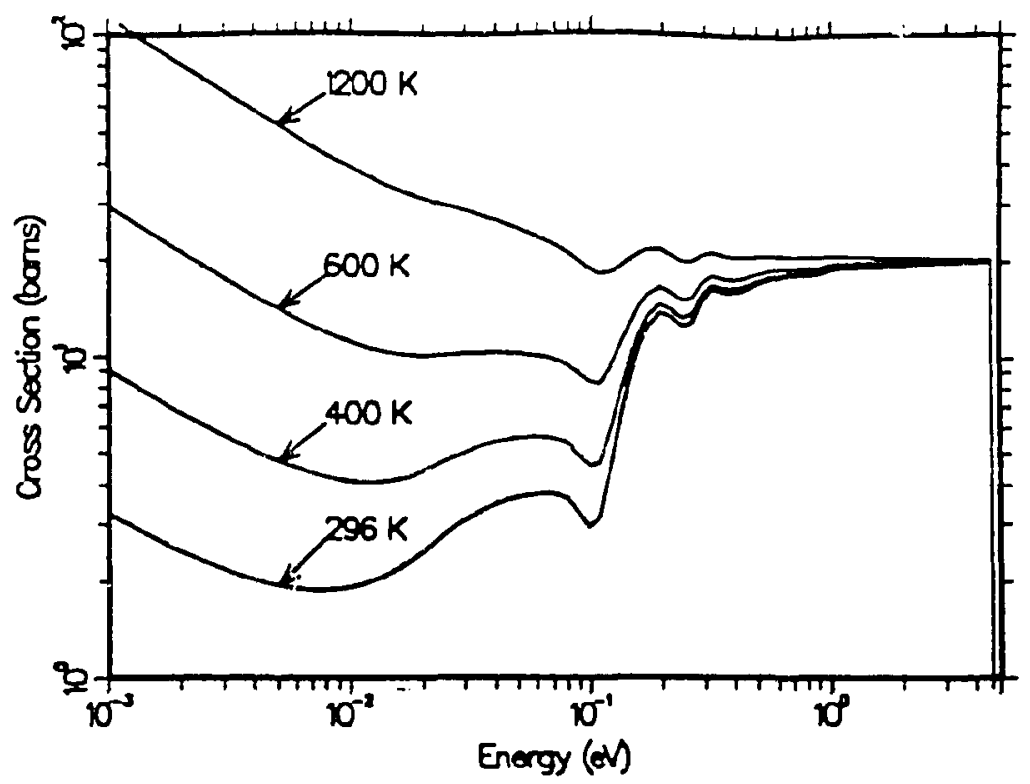

Figure 56: Integrated cross section for $\mathrm{H}$ in $\mathrm{ZrH}$ at four values of the temperaturc. The solid curves are the new evaluation, and the dashed curves are from ENDF/B-VI.0.

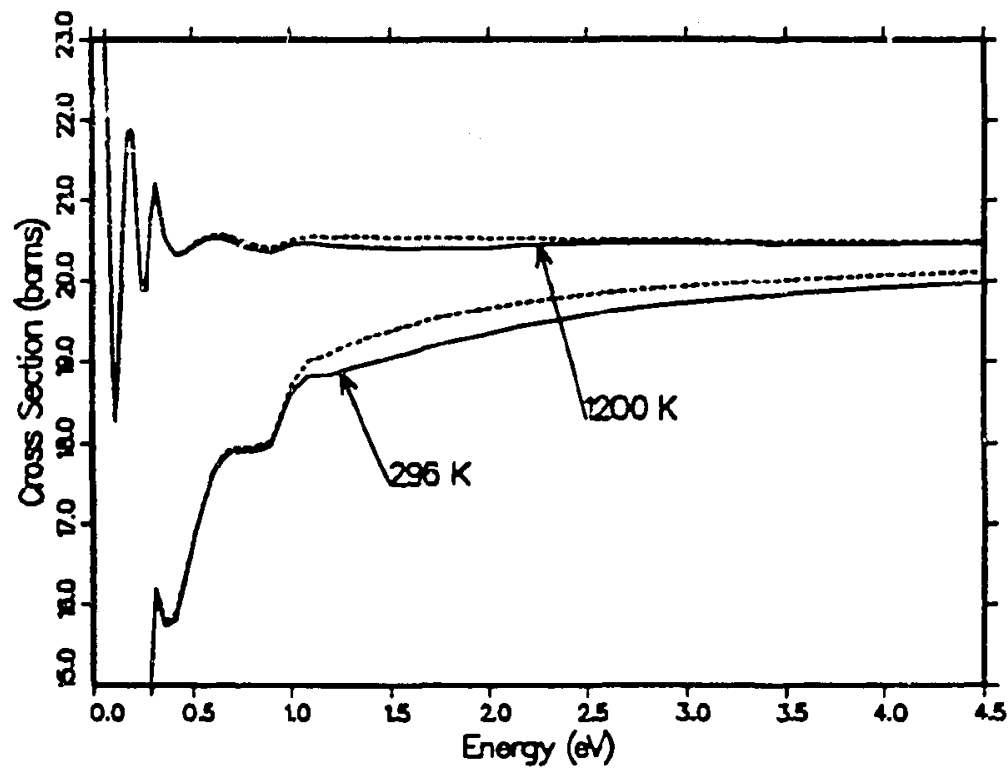

Figure 57: An expanded view of the integrated cross section for $\mathrm{H}$ in $\mathrm{Z} \mathrm{rH}$ at the limiting values of the temperature. The solid curves are the new evaluation, and the dashed curves are from ENDF/B-VI.0. The differences above $\mathrm{leV}$ are due to extending the $\beta$ grid to cover energy transfers as large as $2 \mathrm{eV}$. 


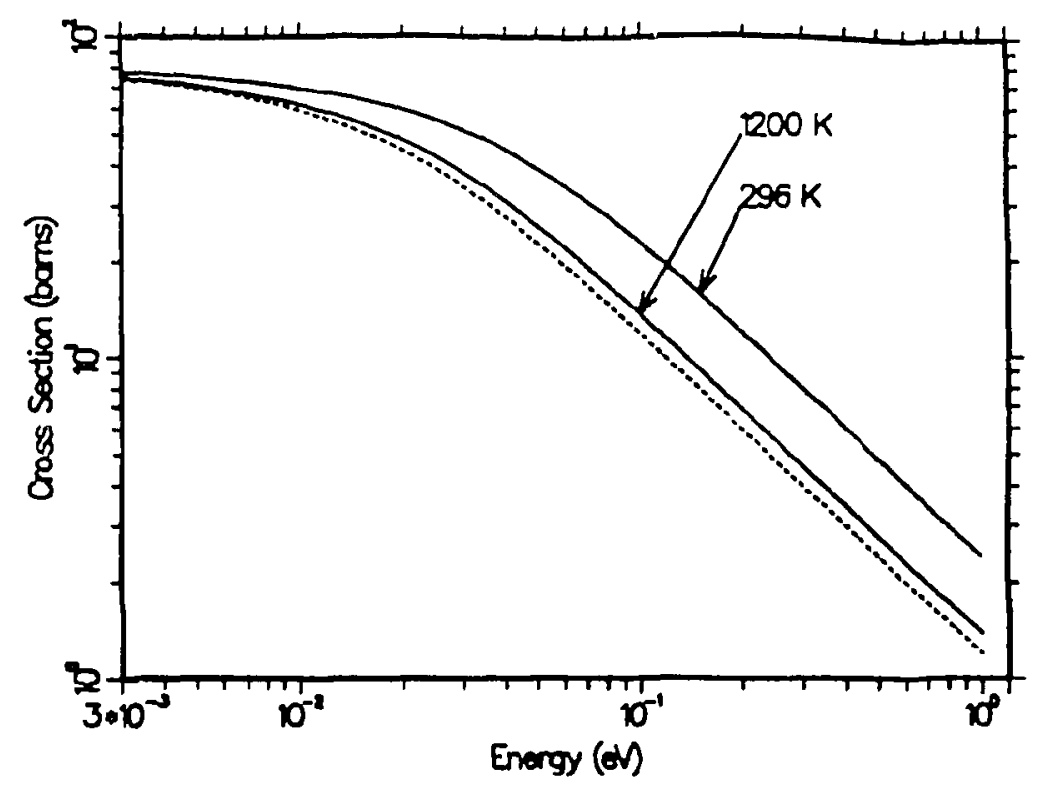

Figure 58: Incoherent elastic cross section for $\mathrm{H}$ in $\mathrm{ZrH}$ at two values of the temperature. The solid curves are the new evaluation, and the dashed curves are from ENDF/B-VI.0.

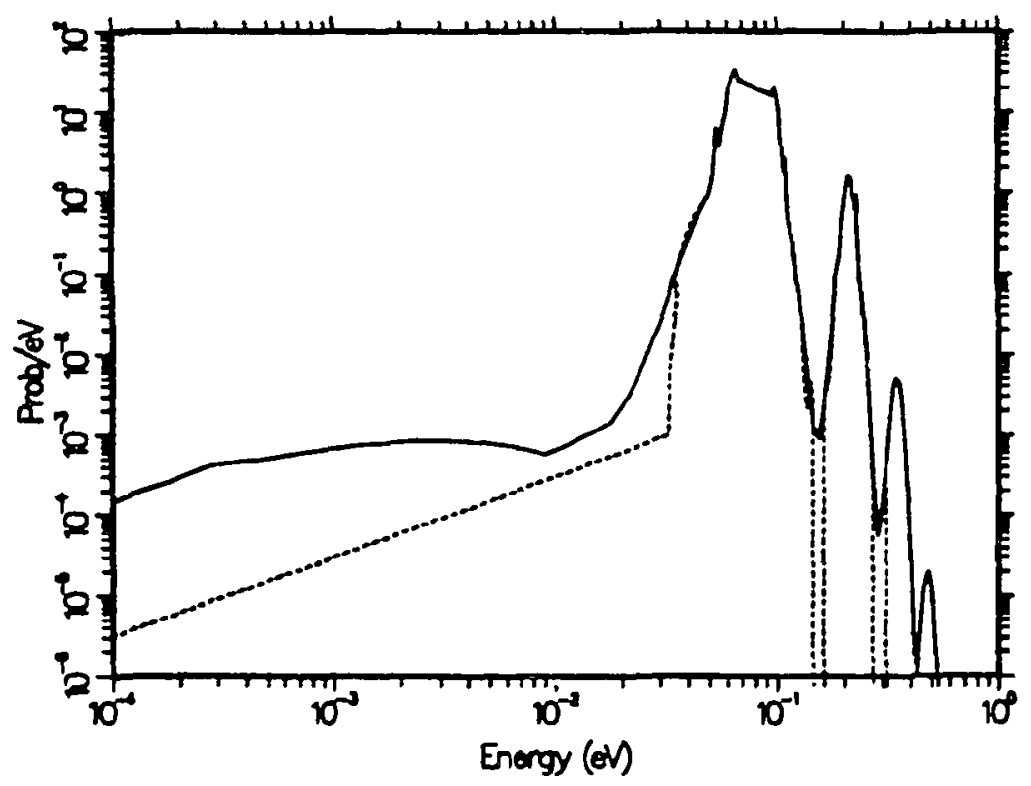

Figure 59: Secondary neutron spectrum from $\mathrm{H}$ in $\mathrm{ZrH}$ for an incident energy of $.0819 \mathrm{eV}$. The solid curves are the new evaluation, and the dashed curves are from ENDF/B-VI.0. 


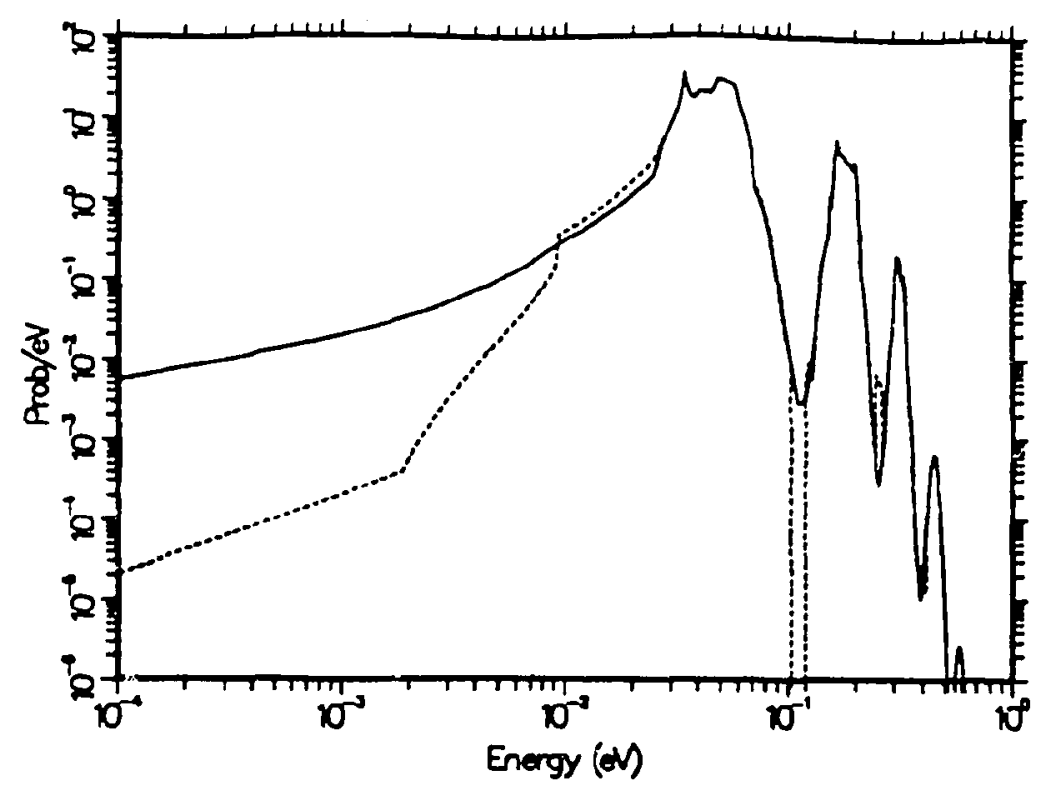

Figure 60: Secondary neutron spectrum from $\mathrm{H}$ in $\mathrm{ZrH}$ for an incident energy of .1844 eV. The solid curves are the new evaluation, and the dashed curves are from ENDF/B-VI.0.

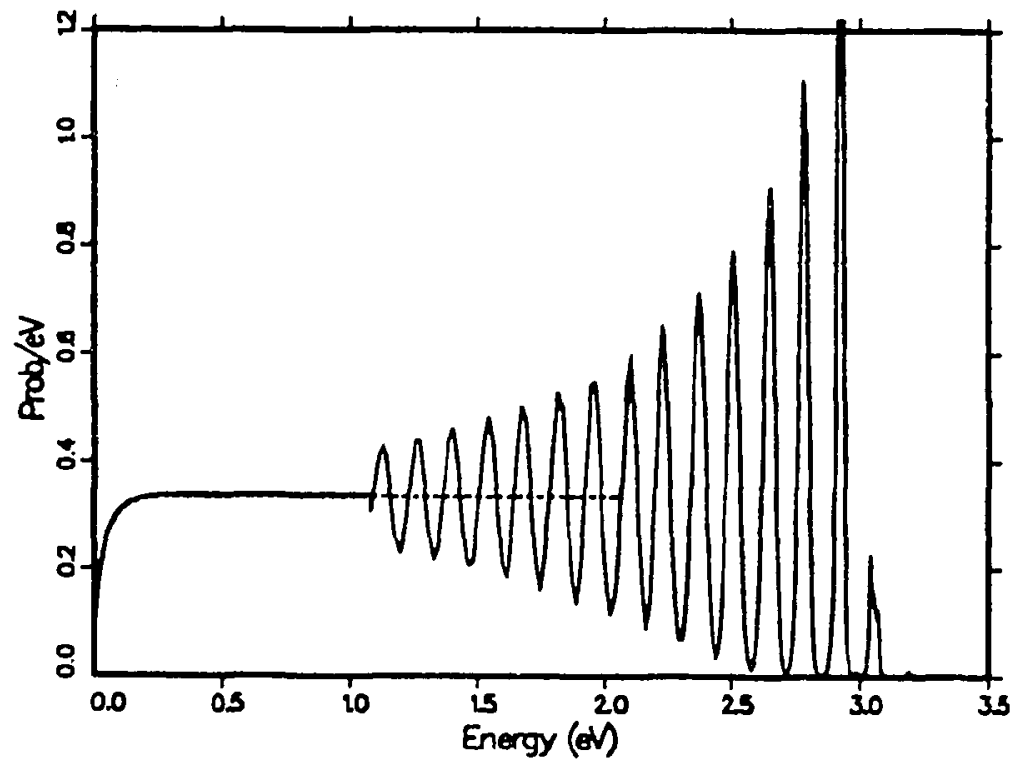

Figure 61: Secondary neutron spectrum from $\mathrm{H}$ in $\mathrm{ZrH}$ for an incident energy of $3.059 \mathrm{eV}$. The solid curves are the new evaluation, and the dashed curves are from ENDF/E-VI.0. Note that energy transfers as large as $2 \mathrm{eV}$ are now treated in detail. 
The same lattice dynamical model can be used to derive a frequency spectrum for $\mathrm{Zr}$ in $\mathrm{ZrH}$. Because of the large mass of the zirconium atom, the maximum energy loss in scattering is small, and it is not necessary to extend the $\beta$ grid. The following input file was used.

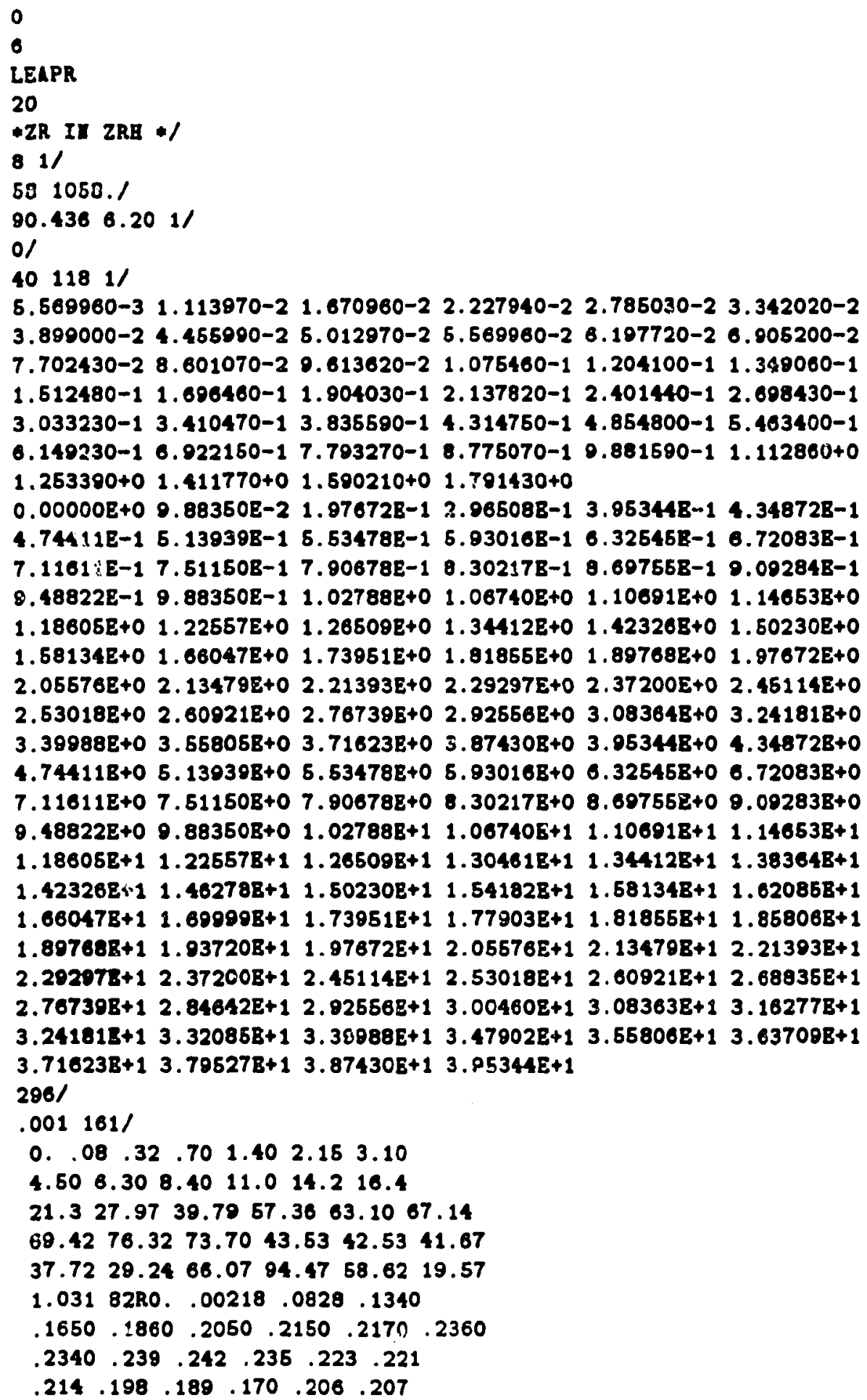




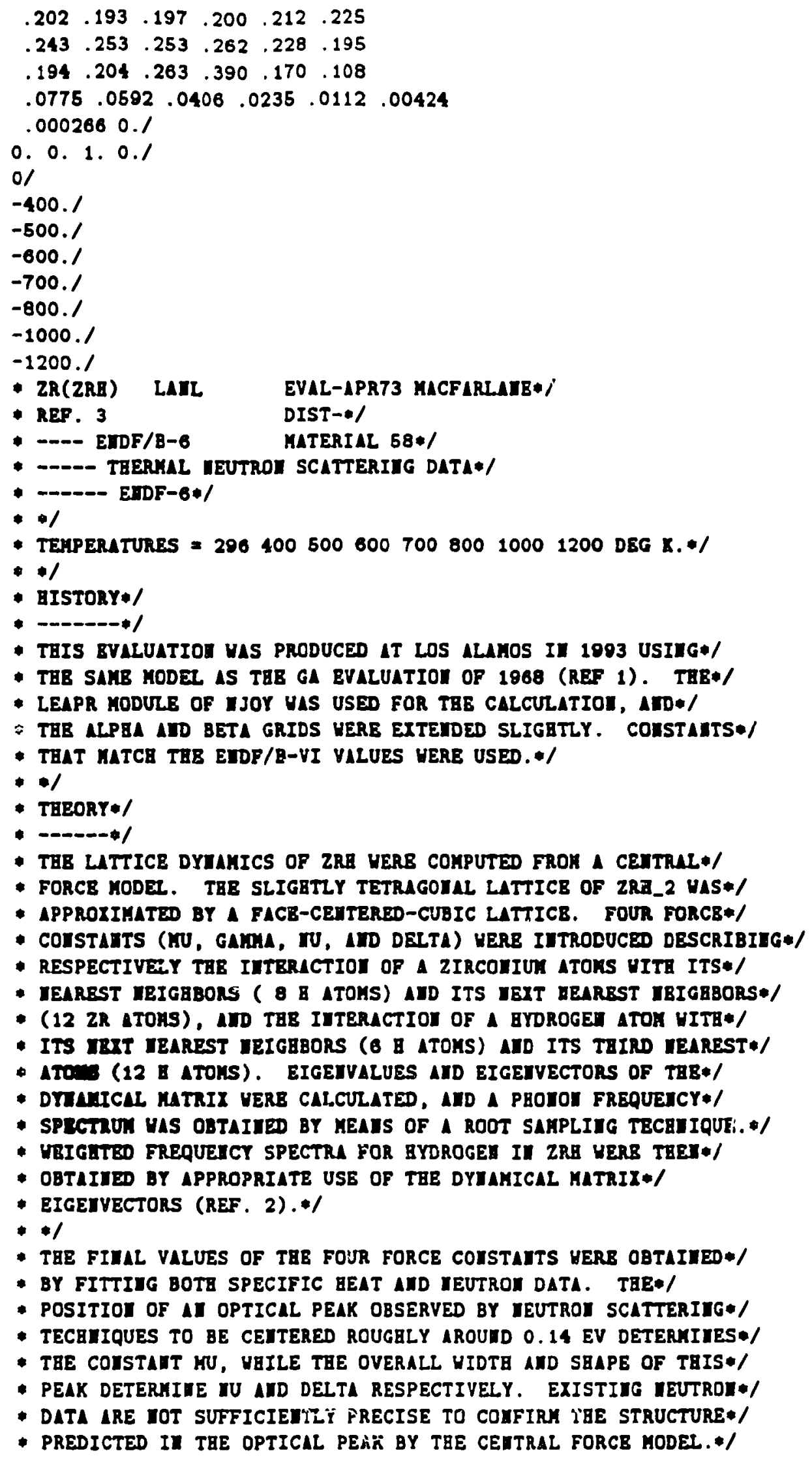




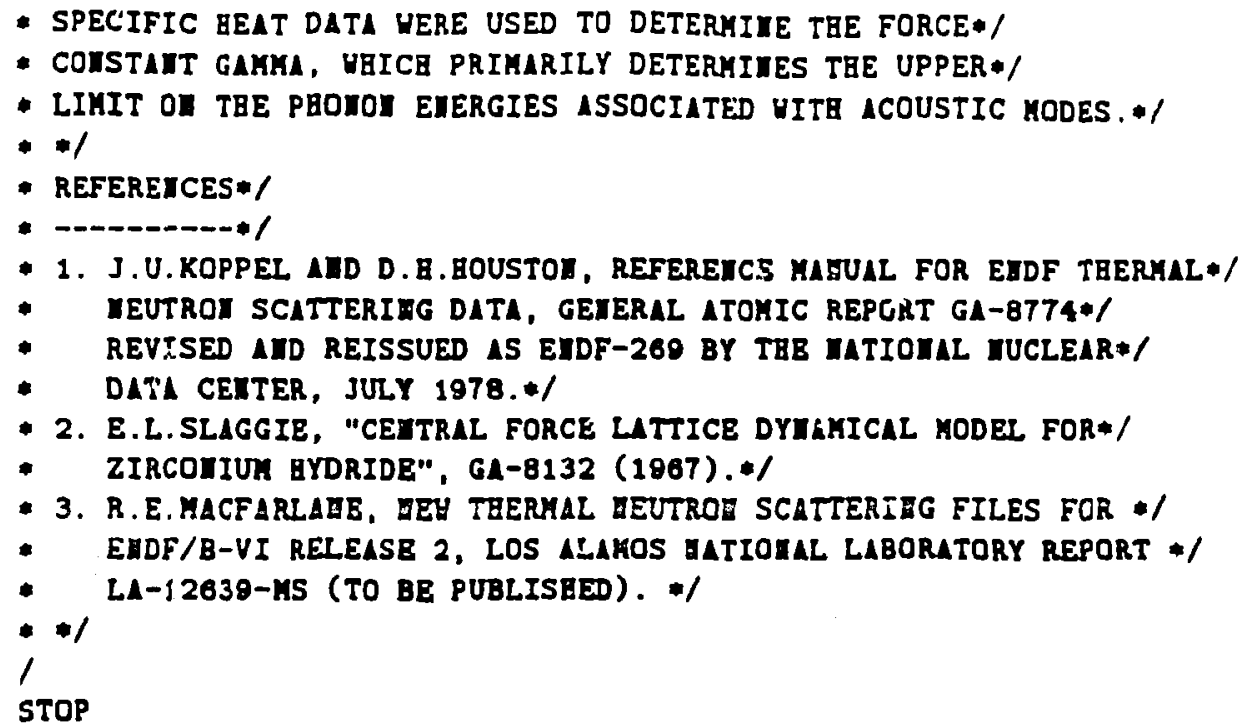

The frequency spectrum from this input file is plotted in Figures 62 and 63 for acoustic modes and optical modes, respectively.

Figures 64 and 65 show the new. and old versions of the scattering law; they are essentially identical. Similarly, the inelastic cross sections shown in Figs. 66 and 67 are basically identical. Figure 68 shows that there was a problem in the incoherent elastic data used for ENBDF/B-VI.0. The Debye-Waller integral values were too large by a factor of $A$, the $\mathrm{Zr}$ mass ratio.

Figures 69 through 71 show secondary neutron spectra for three different incident energies. The agreement between the new and old evaluations is generally good, except for small values of the scattering cross section and for details in the sharp peaks that show ip near $E^{\prime}=E$ for large incident energies.

A table of the effective temperatures and Debye-Waller integrals for both $\mathrm{H}$ and $\mathrm{Zr}$ in $\mathrm{ZrH}$ is given at the end of this section. 


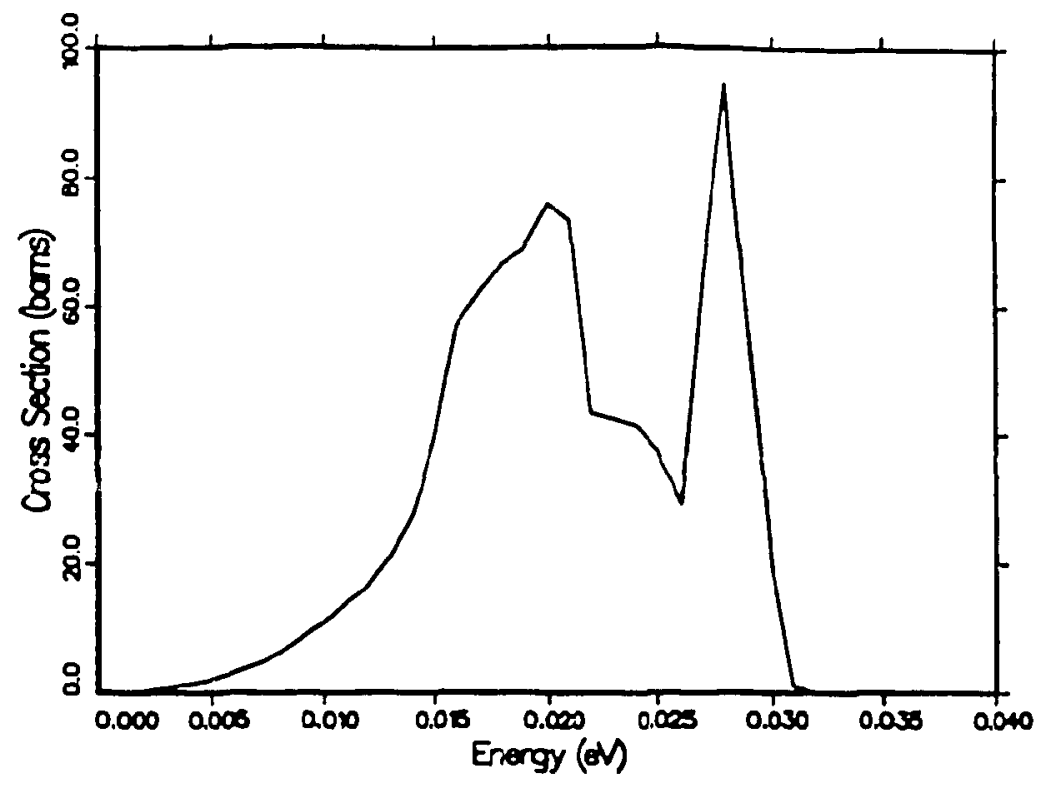

Figure 62: The frequency spectrum used for $\mathrm{Zr}$ in $\mathrm{ZrH}$ showing the acoustic modes.

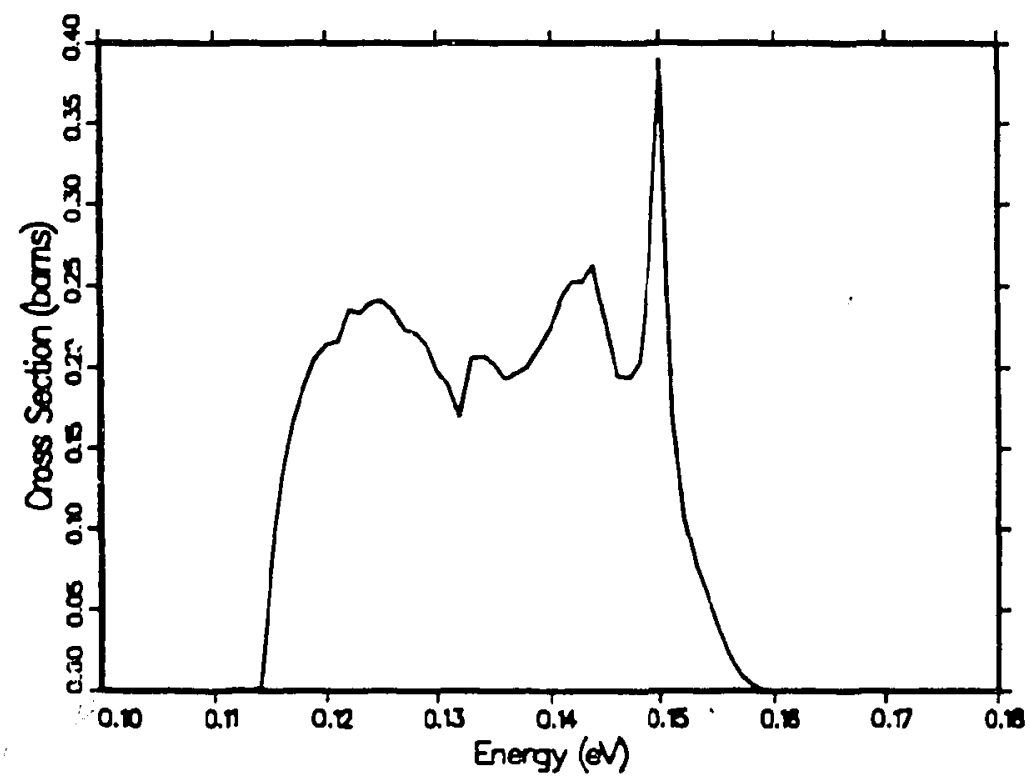

Figure 63: The frequency spectrum used for $\mathrm{Zr}$ in $\mathrm{ZrH}$ showing the optic modes. The function is zero between the top of the acoustic modes and the bottom of the optic modes. 


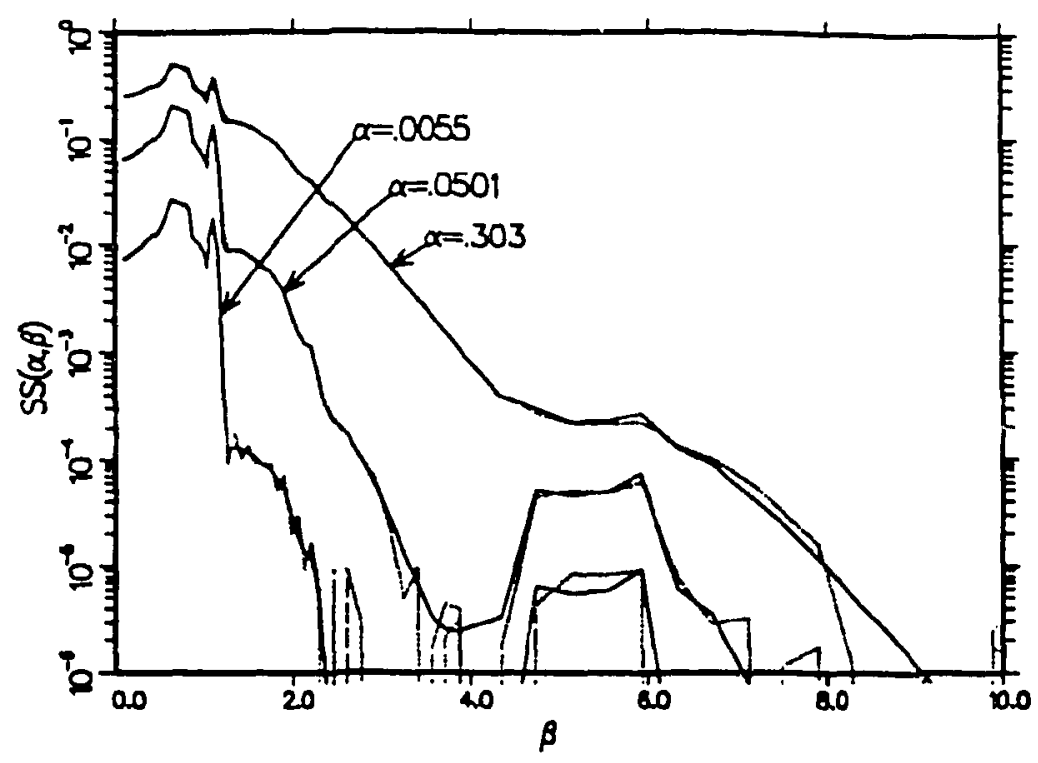

Figure 64: $\mathcal{S}(\alpha,-\beta)$ vs $\beta$ for several $\alpha$ values at a temperature of 296 $\mathrm{K}$ for $\mathrm{Zr}$ in $\mathrm{ZrH}$. The solid lines are the results of this calculation, and the dashed lines are for ENDF/B-VI.0.

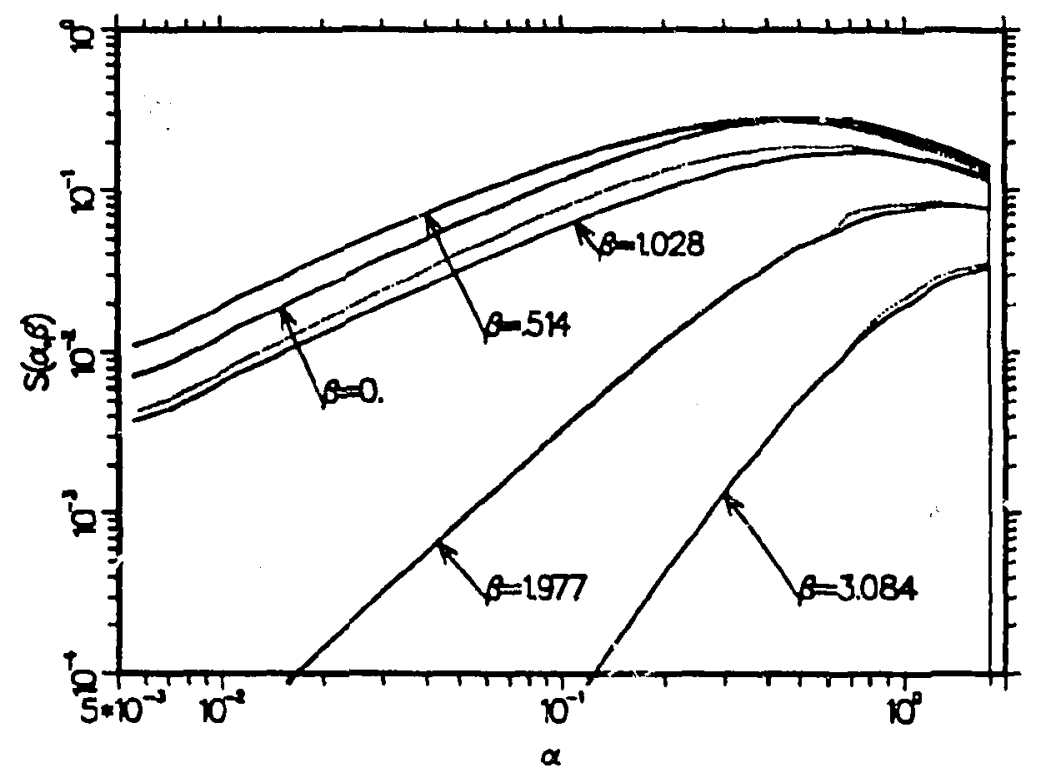

Figure 65: $S(\alpha, \beta)$ vs $\alpha$ for several $\beta$ values at a temperature of 296 $\mathrm{K}$ for $\mathrm{Zr}$ in $\mathrm{ZrH}$. The solid lines are the results of this calculation, and the dashed lines are for ENDF/B-VI.0. 


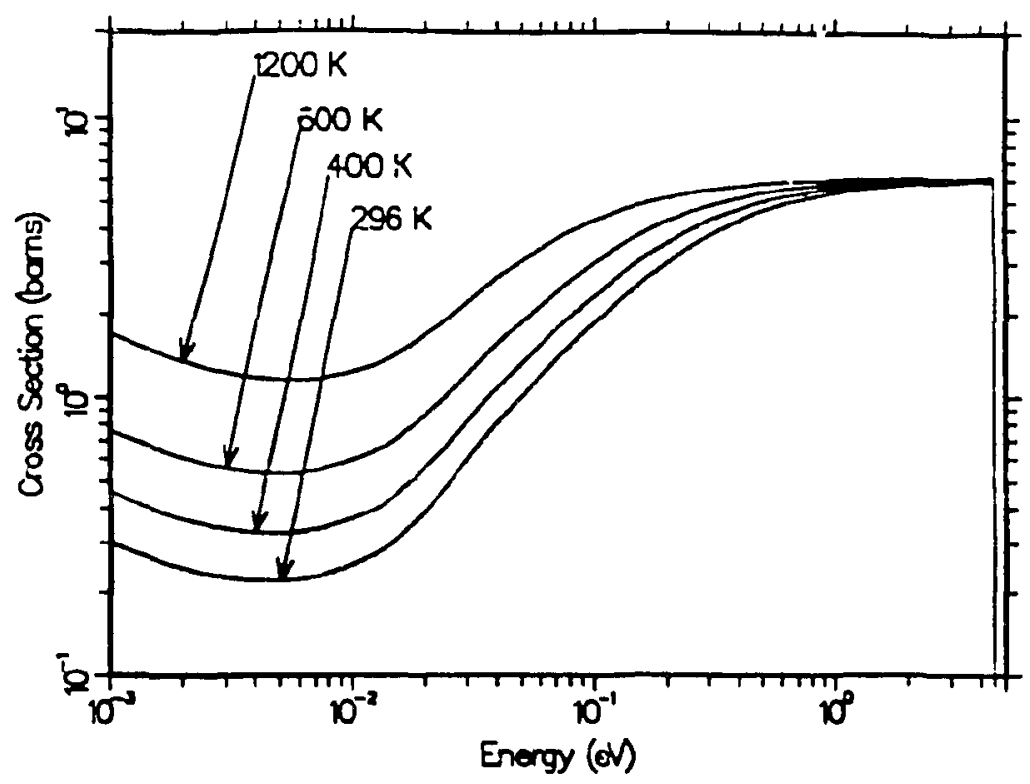

Figure 66: Integrated cross section for $\mathrm{Zr}$ in $\mathrm{ZrH}$ at four values of the temperature. The solid lines are the results of this calculation. The dashed lines for ENDF/B-VI.0 are almost completely hidden by the solid lines.

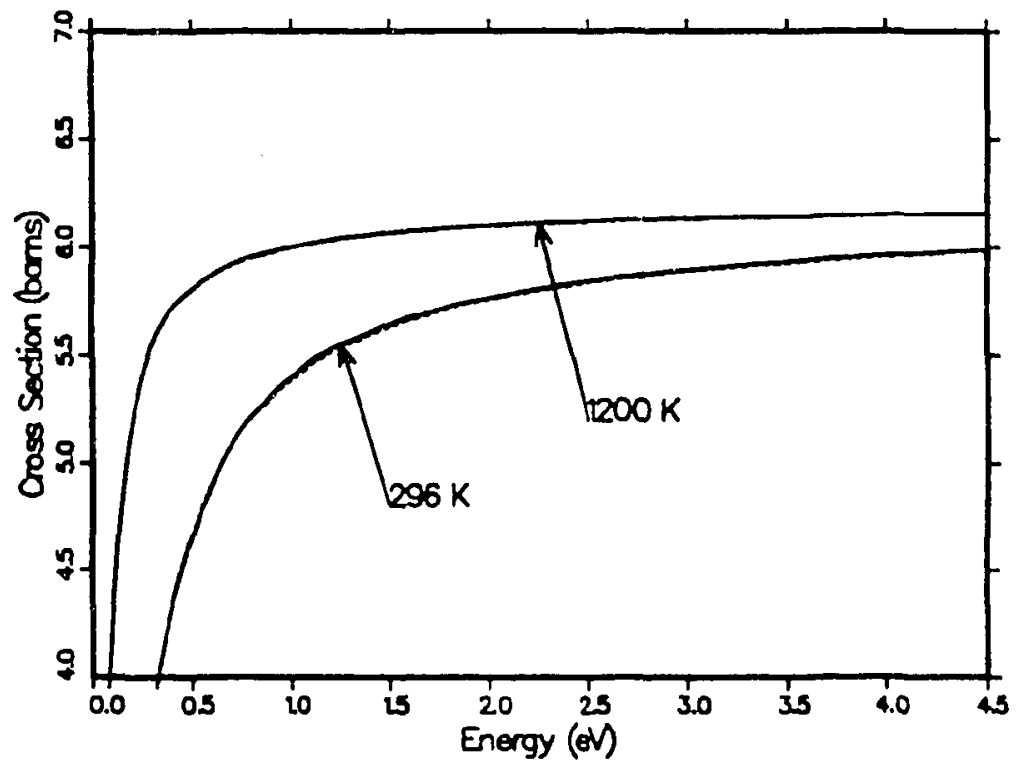

Figure 67: Expinded view of the integrated cross section for $\mathrm{Zr}$ in $\mathrm{ZrH}$ at the limiting values of the temperature. The solid lines are the results of this calculation, and the dashed lines are for ENDF/B-VI.0. 


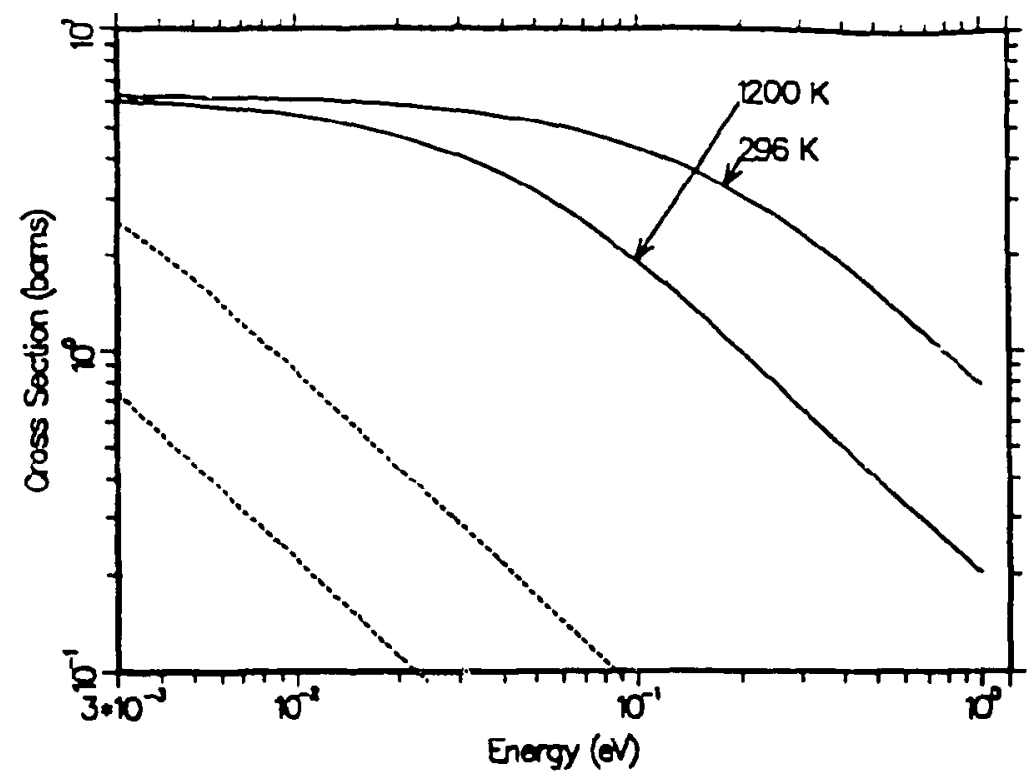

Figure 68: Incoherent elastic cross section for $\mathrm{Zr}$ in $\mathrm{ZrH}$ at two values of the temperature. The solid lines are the results of this calculation, and the dashed lines are for ENDF/B-VI.0.

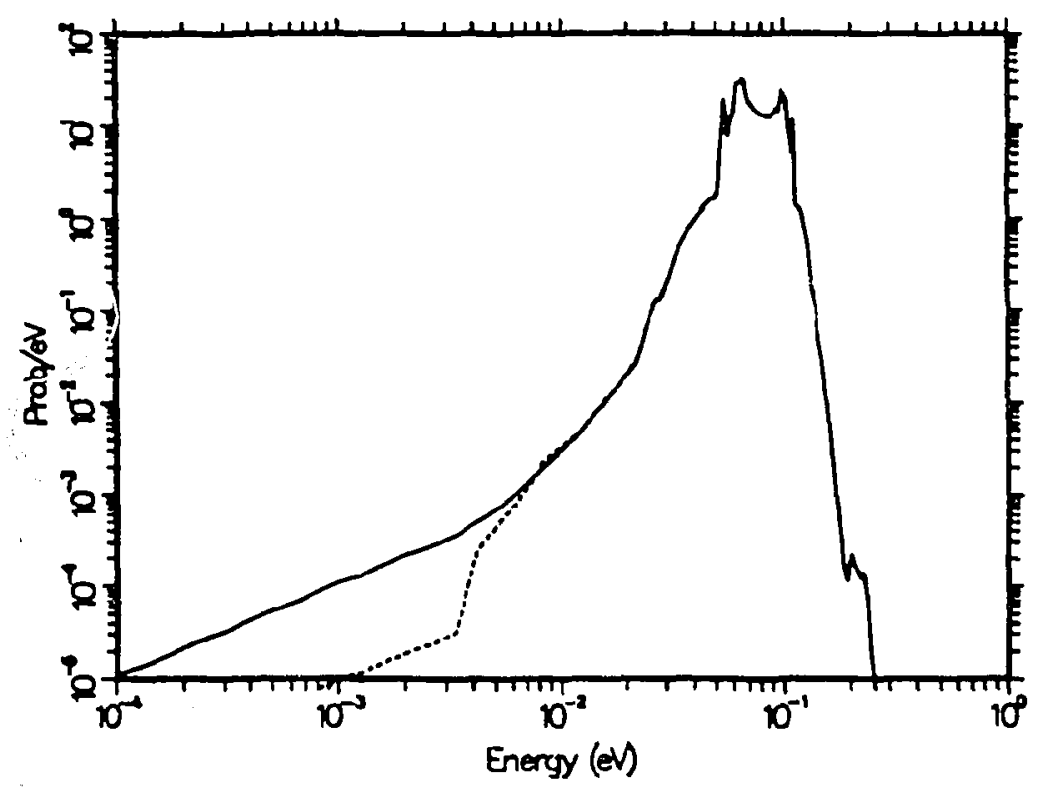

Figure 69: Secondary neutron spectrum frorn $\mathrm{Zr}$ in $\mathrm{ZrH}$ for an incident energy of $.0819 \mathrm{eV}$. The solid lines are the results of this calculation, and the dashed lines are for ENDF/B-VI.0. 


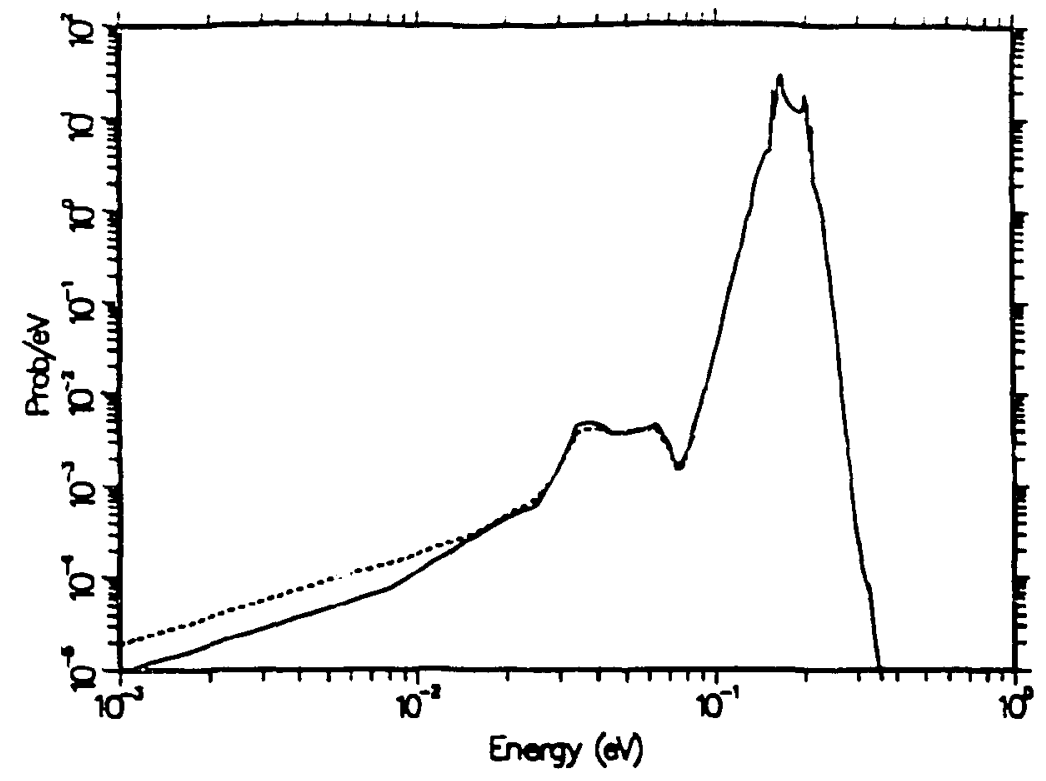

Figure 70: Secondary neutron spectrum from $\mathrm{Zr}$ in $\mathrm{ZrH}$ for an incident energy of $.1844 \mathrm{eV}$. The solid lines are the results of this calculation, and the dashed lines are for ENDF/B-Vi.0.

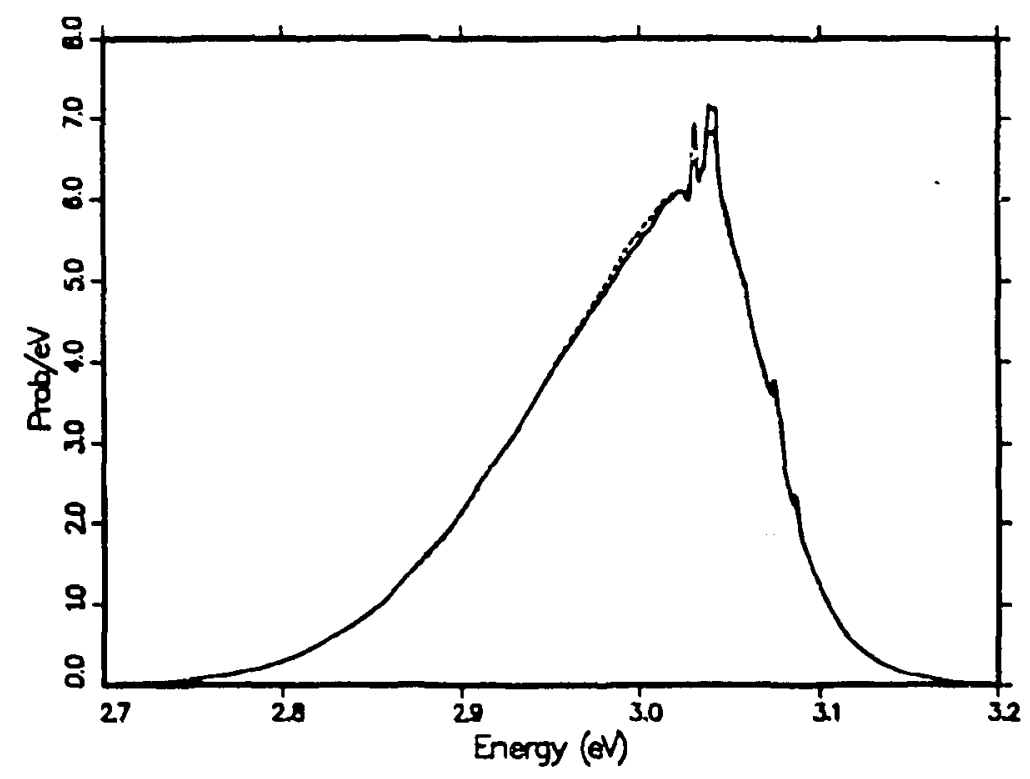

Figure 71: Secondary neutron spectrum from $\mathrm{Zr}$ in $\mathrm{ZrH}$ for an incident energy of $3.059 \mathrm{eV}$. The solid lines are the results of this calculation, and the dashed lines are for ENDF/B-VI.O. 
Table 6: Debye-Waller Integrals and Effective Temperatures for $\mathrm{ZrH}$

\begin{tabular}{ccccc}
\hline $\begin{array}{c}\text { Temperature } \\
\text { (deg K) }\end{array}$ & $\begin{array}{c}\mathrm{H} \text { in } \mathrm{ZrH} \\
\text { Debye-Waller } \\
\text { Int. }\left(\mathrm{eV}^{-1}\right)\end{array}$ & $\begin{array}{c}\mathrm{H} \text { in } \mathrm{ZrH} \\
\text { Eff. Temp. } \\
\text { (deg K) }\end{array}$ & $\begin{array}{c}\text { Zr in ZrH } \\
\text { Debye-Waller } \\
\text { int. }\left(\mathrm{eV}^{-1}\right)\end{array}$ & $\begin{array}{c}\text { Zr in ZrH } \\
\text { Eff. Temp. } \\
\text { (deg K) }\end{array}$ \\
\hline 296 & 8.480 & 806.7 & 182.1 & 317.4 \\
400 & 9.086 & 829.9 & 242.2 & 416.3 \\
500 & 9.820 & 868.4 & 300.6 & 513.3 \\
600 & 10.68 & 920.0 & 359.3 & 611.2 \\
700 & 11.63 & 981.8 & 418.2 & 709.6 \\
800 & 12.64 & 1051. & 477.2 & 808.5 \\
1000 & 14.82 & 1205. & 595.4 & 1007. \\
1200 & 17.13 & 1373. & 713.7 & 1206. \\
\hline
\end{tabular}




\section{SOLID METHANE}

The methane molecule consists of an atom of carbon surrounded by four atoms of hydrogen placed on the corners of a tetrahedron. The carbon atom is at the center of mass of the system; beccuse of its symmetry, the methane molecule is often called a "spherical top". Optical measurements of methane in the gas phase show four fairly well defined vibrational modes at 162, 190, 361 , and $374 \mathrm{meV}$. Following the lead of Picton, they have been included in this model as discrete oscillators with weights equal to $.308, .186, .042$, and .144 , respectively.

Specific heat measurements in solid methane near one atmosphere show three phases with transitions at $8 \mathrm{~K}$ and $20.4 \mathrm{~K}$. The melting point is about $89 \mathrm{~K}$. Xray measurements show that the carbon atoms are arranged on a face-centered cubic (fcc) lattice for both of the higher two phases; it has been speculated that the phase transition is due to a change in the degree of rotational order, or perhaps due to the onset of a self-diffusion behavior. Because of this interesting question, slow neutron inelastic scattering experiments were carried out with samples in each of the phases. ${ }^{20}$ Because hydrogen is an incoherent scatterer, it was possible to analyze the data to obtain a frequency spectrum for hydrogen in solid methane. The results didn't really explain what was happening in the 20 $\mathrm{K}$ phase transition, but they did provide us with just the data needed for our calculation.

Again following Picton, we chose the spectrum for $22.1 \mathrm{~K}$ for our model. Instead of using Picton's numbers directly, we digitized the curve from the graph in the reference, plotted it on a large scale, and then smoothed it by hand. Care was taken to use an $\epsilon^{2}$ varizition for low energies. As discussed by Harker and Brugger, the appropriate normalization for this frequency spectrum is 0.32 . This spectrum and the four discrete oscillators were then used to calculate $S(\alpha, \beta)$ with LEAPR using the $\alpha$ and $\beta$ grids of Picton. The input file follows:

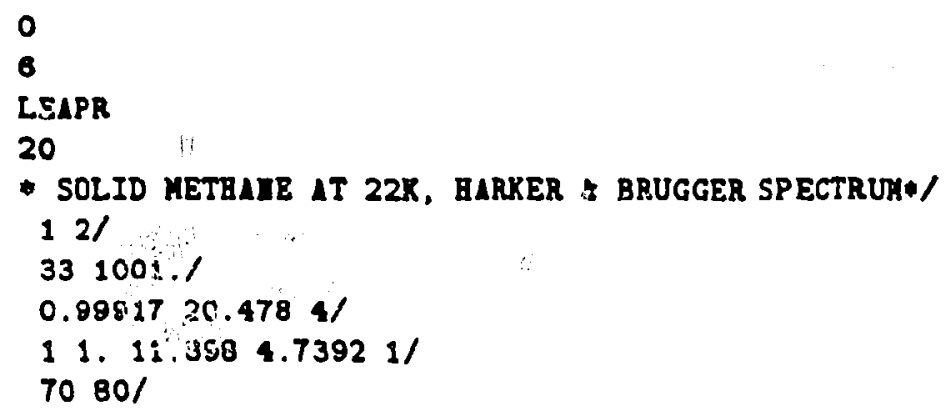




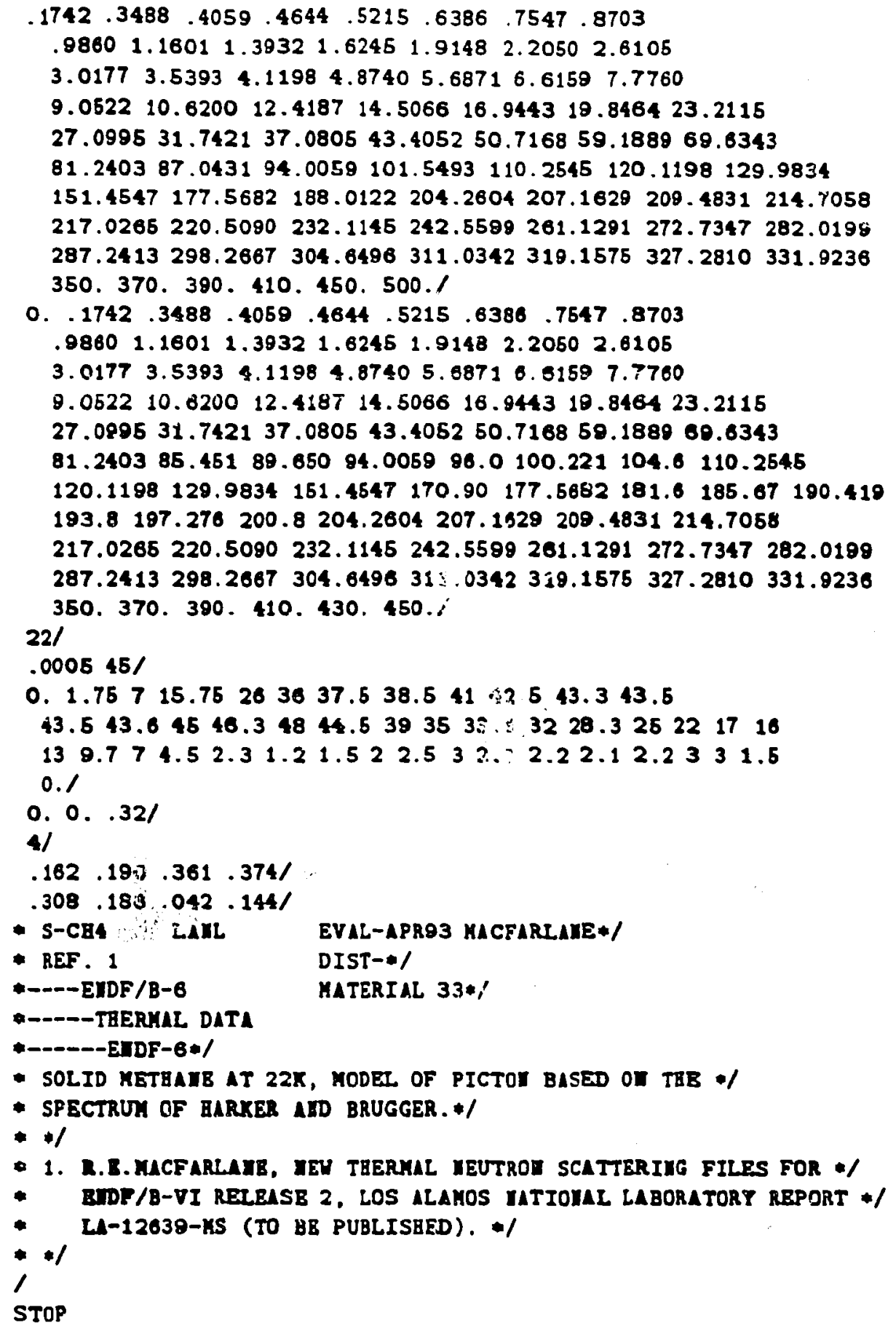

The frequency spectrum given in this input file is plotted in Figure 72. During the calculation, the moments of $T_{n}$ and $S(\alpha, \beta)$ were checked and the errors were modest. The output listing for the solid-type part of the problem was examined carefully to see that the $\alpha$ and $\beta$ ranges were sufficient and that the normalization and sum-rule checks were reasonably well satisfied. Since this is a solid, there was no translational calculation. In the discrete-oscillator calculation the delta 


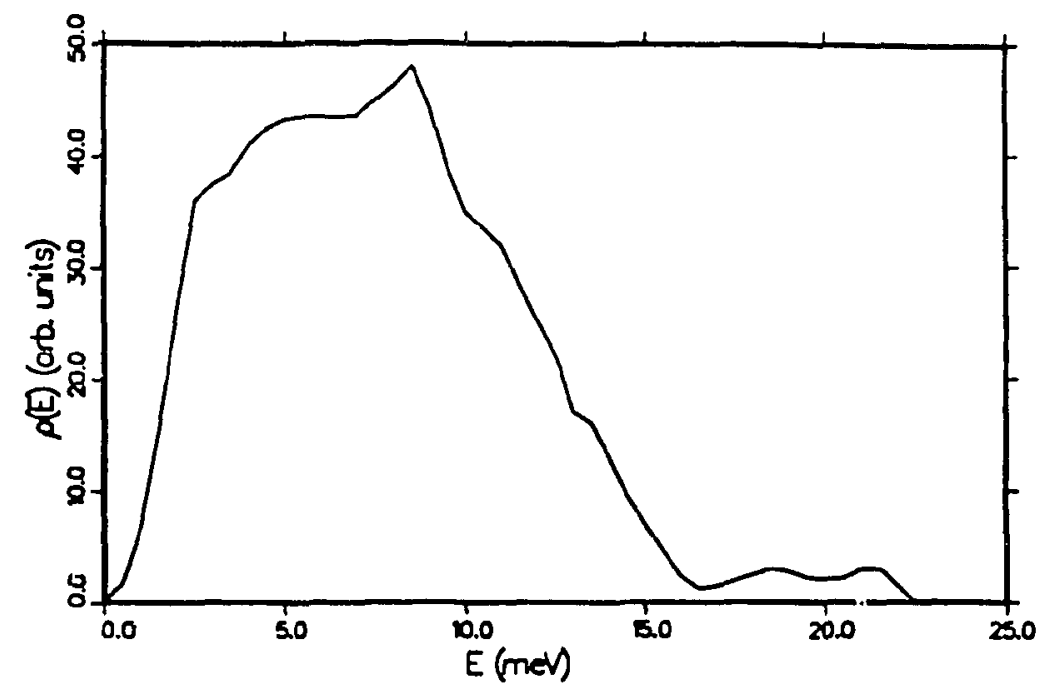

Figure 72: The Harker-Brugger frequency spectrum used for solid methane. Note the quadratic shape at low energies.

functions for $\beta>0$ were put directly into the scattering law as sharp triangles. The $\beta=0$ peak was converted into incoherent elastic scattering. A portion of the output listing showing the discrete lines is shown below:

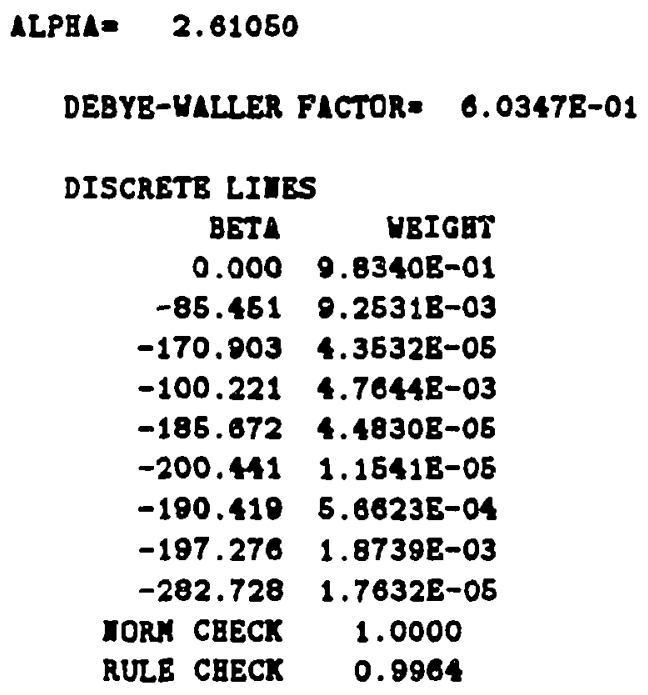

The Debye-Waller factor printed here is the one from the solid-type spectrum, $\lambda_{3}$. It defines the total strength of all the discrete lines. All the discrete lines taken together must satisfy the normalization and sum-rule requirements of any scattering law. If the number of discrete lines increases above 100 , some of them 


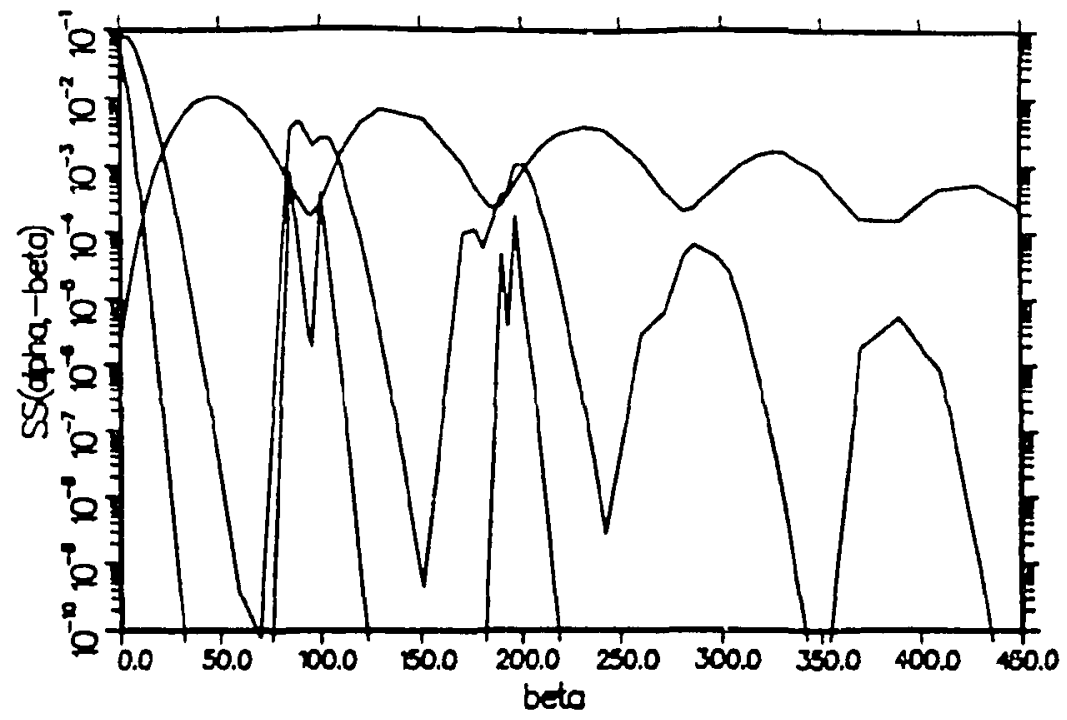

Figure 73: $\mathcal{S}(\alpha,-\beta)$ for solid methane shown as a function of $\beta$ for the $\alpha$ values 0.986 (lowest), 16.94, and 151.45 (highest). The four discrete levels show up as sharp triangles in the lowest curve. SS is used to denote script $\mathrm{S}$.

will begin to be lost, and the checks will begin to decrease below unity. The listing for the discrete-oscillator calculation shows that the grid specified does a reasonable job of satisfying the overall normalization and sum-rule checks. LEAPR automatically prepared an output file in ENDF-6 format. Plots of $S(\alpha, \beta)$ versus $\beta$ for several values of $\alpha$ are given in Figure 73 .

Next, the new evaluation for $S(\alpha, \beta)$ was processed into integrated cross secijons and double differential cross sections using the THERMR module of NJOY. When this was first done, it was necessary to slightly modify the NJOY code to allow for the very large values of $\beta$ appropriate to these low temperatures (note that $\beta$ is inversely proportional to $k T$ for a given energy transfer); it is necessary to keep values of $S$ as small as $1 \times 10^{-80}$ for this evaluation. This is quite a dynamic range! $A$ plot of the integrated cross section is given in Figure 74 , and plots of the outgoing neutron spectrum integrated over angle at several incident energies are given in Figure 75.

The output of THERMR can then be used to produce either meltigroup or Monte Carlo cross sections. 


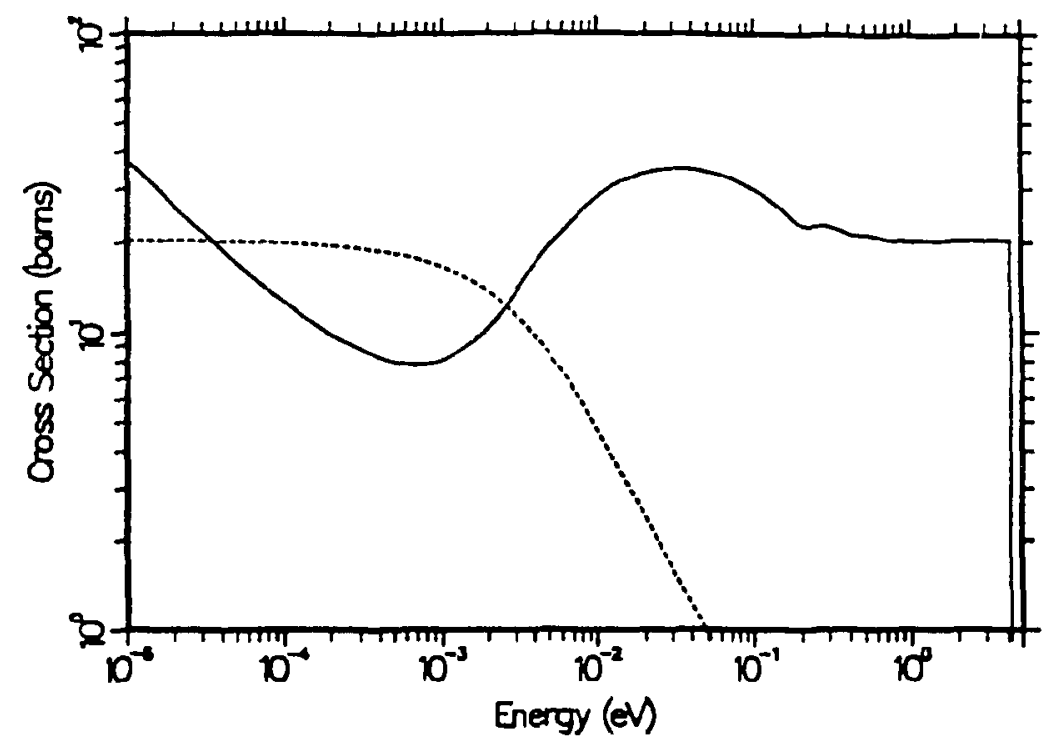

Figure 74: Inelastic (solid) and incoherent elastic (dashed) cross sections for solid methane. The small bumps starting at about $0.2 \mathrm{eV}$ are due to the discrete levels at $.162, .190, .361$, and $.374 \mathrm{eV}$.

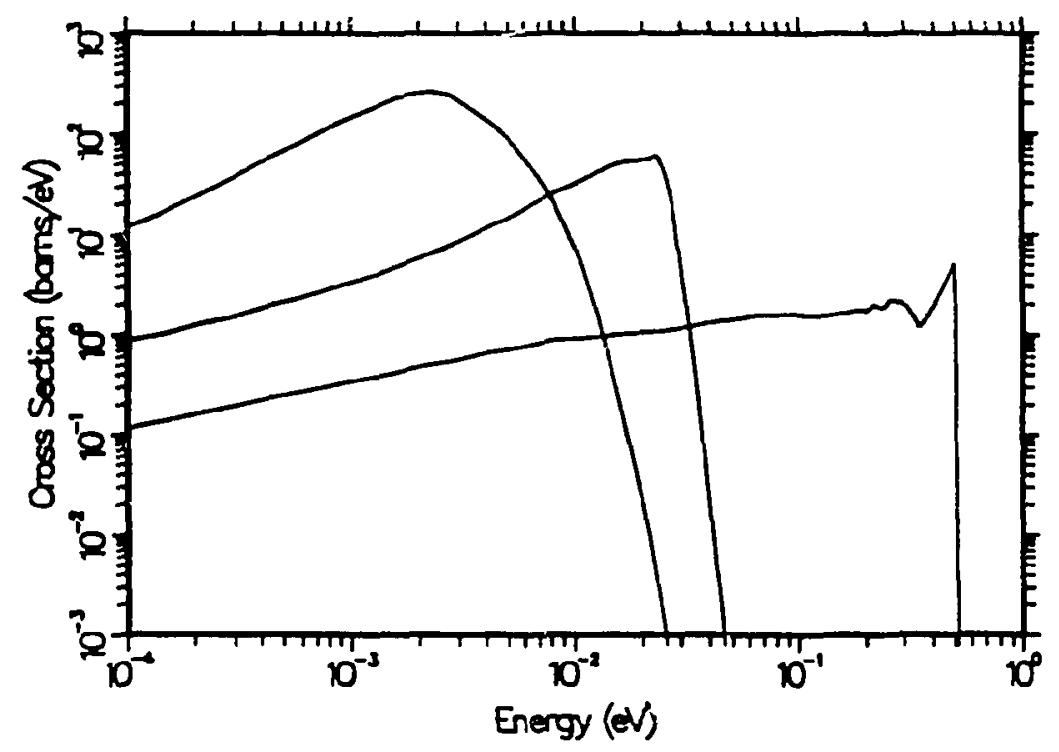

Figure 75: Neutron spectra $\sigma\left(E \rightarrow E^{\prime}\right)$ for solid methane shown as functions of outgoing neutron energy $E^{\prime}$ for $E=0.0001, .0253$, and .503 $\mathrm{eV}$. 


\section{LIQUID MFTHANE}

The preparation of a model for liquid methane at $90 \mathrm{~K}$ was a little more difficult. Once again, we use the four discrete oscillators to represent the molecular vibrations. In addition, we need a continuous frequency distribution to represent the molecular rotations, and a pair of parameters $w_{\xi}$ and $c$ to represent diffusion. This latter component was omitted from Picton's model, but we felt that it might be needed to obtain a reasonable quasi-elastic peak in the spectrum of scattered neutrons. Therefore, we couldn't use the Picton input directly, and we had to refer to his source. ${ }^{21}$ Agrawal and Yip divided the problem into two parts: translations and rotations.

For translations, they proposed a model for $\gamma(t)$ that matches the expected diffusive behavior at long times and provides an oscillatory behavior at short times. Each methane molecule is assumed to move in a "cage" formed by its neighbors, and the cage itself is allowed to relax with time. As Agrawal and Yip point out, the molecule will oscillate initially, but gradually as the restoring forces decay into a frictional background, it will go over into diffusive motions. The resulting analytic expression for the frequency spectrum is

$$
f_{:}(\omega)=\frac{2}{\pi} \frac{\omega_{0}^{2} / \tau_{0}}{\left(\omega^{2}-\omega_{0}^{2}\right)^{2}+\left(\omega / \tau_{0}\right)^{2}} .
$$

The fact that $f(\omega)$ is nonzero at $\omega=0$ indicates that the molecules are capable of diffusion, and in addition, $f(\omega)$ has a resonant behavior near $\left(\omega^{2}-\tau_{0}^{2}\right)^{1 / 2}$, the characteristic frequency of local oscillations.

For rotations, the argument starts out by recalling that for translations, $\gamma(t)$ is related to the mean-square displacement $W(t)$ by

$$
\gamma(t)=\kappa^{2} W(t)
$$

where the magnitude of the wave vector is related to $\alpha$ by

$$
x=\frac{\hbar^{2} \kappa^{2}}{2 M k T} \text {. }
$$

The rotational analog of the mean-square displacement is the mean-square component of the bond length $\vec{b}$ along the vector $\vec{\kappa}$, or

$$
W(t)=\left\langle\left[b_{\kappa}(t)-b_{\kappa}(0)\right]\right\rangle^{2}
$$




$$
\begin{aligned}
& =2<b_{\kappa}^{2}>\left[1-\frac{\left\langle b_{\kappa}(t) b_{\kappa}(0)\right\rangle}{\left.<b_{\kappa}^{2}\right\rangle}\right] \\
& =\frac{2 b^{2}}{3}\left[1-F_{1}(t)\right] .
\end{aligned}
$$

The function $F_{1}(t)$ is seen to describe the correlation between a specific direction in the molecule at $t=0$ with its direction at a later time $t$. Therefore, it is called the "dipole correlation function". The same function appears in the classical limit of the theory of optical line shapes for infrared absorption as presented by Gordon. 22 At frequencies where it is safe to assume that the internal vibrations of different molecules are uncoupled, the shape of a vibrational line depends mostly on the reorientation motions of individual molecules, and the dipole correlation function can be obtained from

$$
F_{1}(t)=\int_{\text {band }} \hat{I}(\omega) \cos \omega t d \omega
$$

Gordon has used this inethod to compute $F_{1}(t)$ for liquid methane at $98 \mathrm{~K}$ based on the infrared data of Ewing. ${ }^{23}$ In order to link this result to neutron scattering, we use the high-temperature classical limit of Eq.(5) to express $W(t)$, namely,

$$
W(\hat{t})=\frac{\hbar^{2}}{2 M k T} \int_{0}^{\infty} \frac{\rho_{r}}{\beta^{2}}[1-\cos (\beta \hat{t})] d \beta,
$$

which can be inverted to obtain

$$
\rho_{r}(\beta)=\frac{2 M k T}{\hbar^{2}} \int_{-\infty}^{\infty} \frac{d^{2} \gamma_{r}}{d t^{2}} e^{i \beta \hat{t}} d \hat{t}
$$

This limit is justified by noting that $\beta<1$ for the rotational modes in liquid methane around $90 \mathrm{~K}$. It is now easy to compute $\rho_{r}$ by taking two derivatives of the dipole correlation function graphed by Gordon.

The result is shown in Figure 76, together with the translational frequency distribution discussed above. These numbers were generated by digitizing the curve from Agrawal and Yip, subtracting the translational part, and smoothing the remainder. Agrawal and Yip compared their model with both double-differential and integrated cross sections, with very good agreement.

Unfortunately, this model does not match the requirements of LEAPR. The only type of frequency distribution that is nonzero at $\omega=0$ that can be used by the 
code is the diffusive law of Egelstaff and Schofield, which does not have the shorttime oscillatory behavior of Eq.(73). Our main reason for using the diffusion term in our model for liquid methane was to improve the "quasi-elastic" peak, which depends mostly on the small- $\omega$ part of the frequency distribution. Therefore, it seemed reasonable to select diffusion parameters $w_{t}$ and $c$ that gave a reasonable representation for the full width at half maximum of the quasi-elastic peak, to subtract the result $f_{d}$ from the sum of the two curves shown in Figure 76, and to use the difference to recresent both the translational oscillatory modes and the rotational modes. Figr:a 77 shows this breakdown. Once again, there has been some hand smoothing, and the low energy part of the distribution was forced to follow an $\epsilon^{2}$ law. The final breakdown was $1.5 \%$ diffusion, $30.5 \%$ rotation, and $68 \%$ molecular vibrations.

The LEAPR input for liquid methane at $90 \mathrm{~K}$ is shown below.

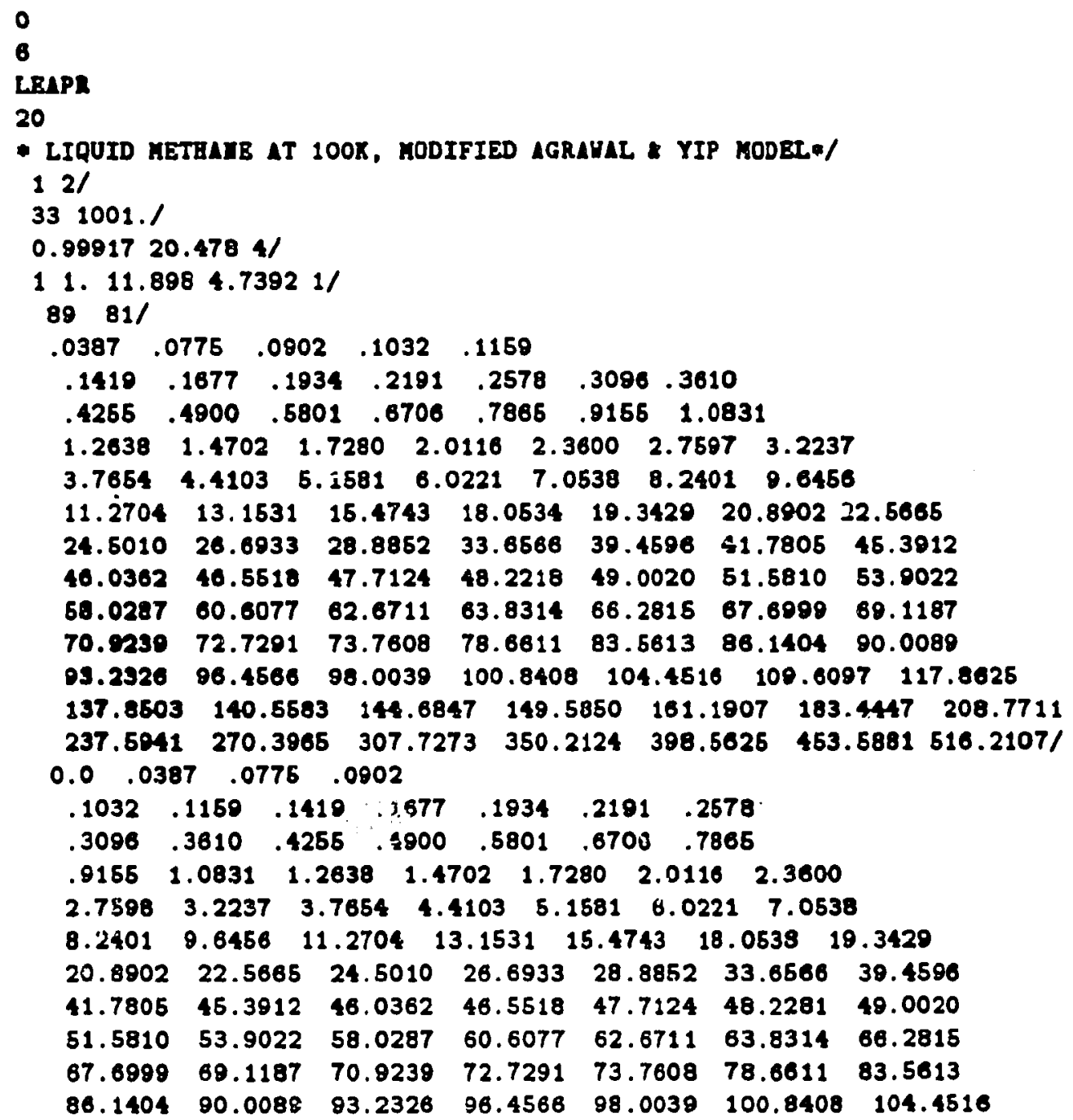




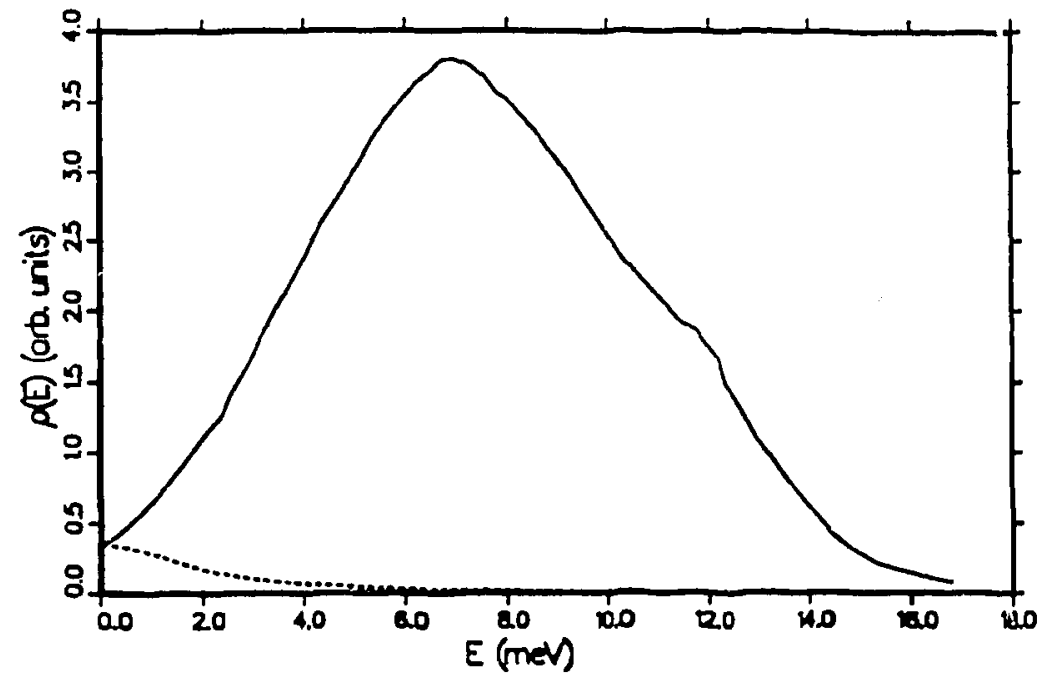

Figure 76: Frequency spectrum for liquid methane (solid) as given by Agrawal and Yip, including an analytic translational part (dashed) and a rotational part based on Gordon's analysis of the optical measurements of Ewing.

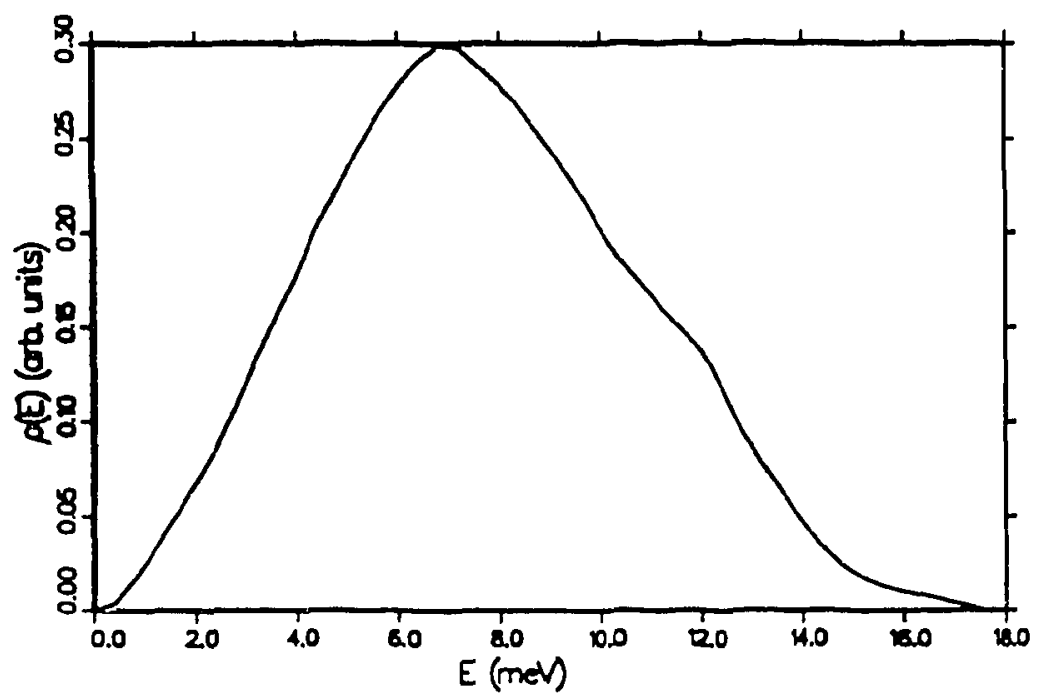

Figure 77: Effective frequency spectrum for methane including both translational and rotational modes, but not including diffusive modes. 


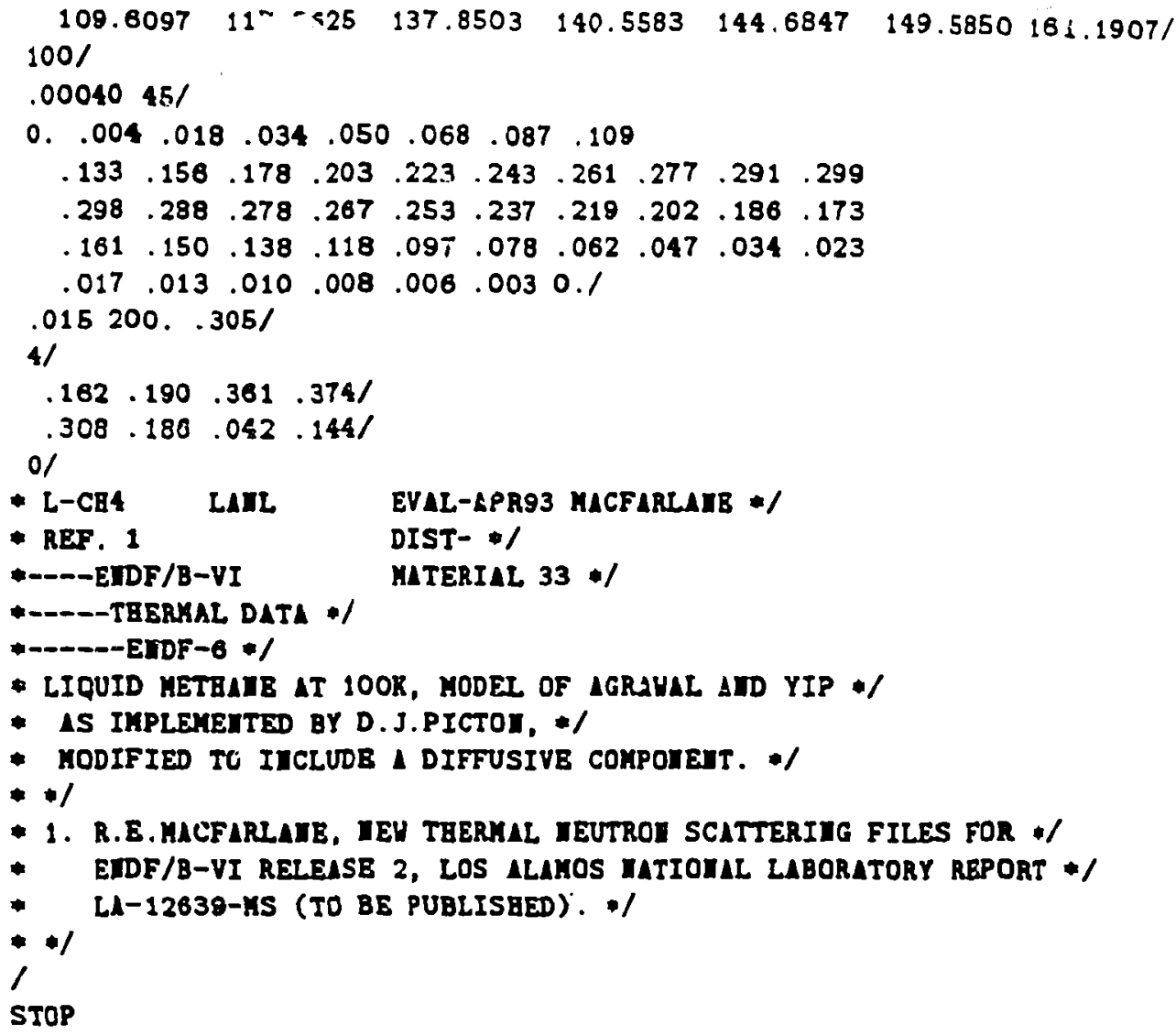

LEAPR was run with this input taking advantage of the much acrelerated diffusion calculation discussed above. Once again the moments of $T_{n}$ and $S(\beta)$ were checked, and no great problems were seen. These checks help to prove that the $\epsilon$ grid for the input frequency spectrum and the $\beta$ grid for calculating $S$ are reasonable. We also checked the range of $\alpha$ and $\beta$ to be sure that no significant cross section contributions were being cut off. The results seem to be good for all energy transfers possible with incident neutron energies up to $1 \mathrm{eV}$. Once again, LEAPR produced an output file in ENDF-6 lormat. This time, there was no elastic contribution at all. Plots of $S(\alpha, \beta)$ versus $\alpha$ for several values of $\beta$ are shown in Figure 78. Note that the behavior of the curves for small $\beta$ is quite different than in Figure 73. This reflects the presence of the diffusive component.

The new evaluation for liquid methane was run through the THERMR module of NJOY to produce integrated and differential cross sections. Sample results are given in Figures 79 and 80 . The integrated cross section is compared with experimental data at $110 \mathrm{~K}$ that was quoted in the Agrawal and Yip paper. 


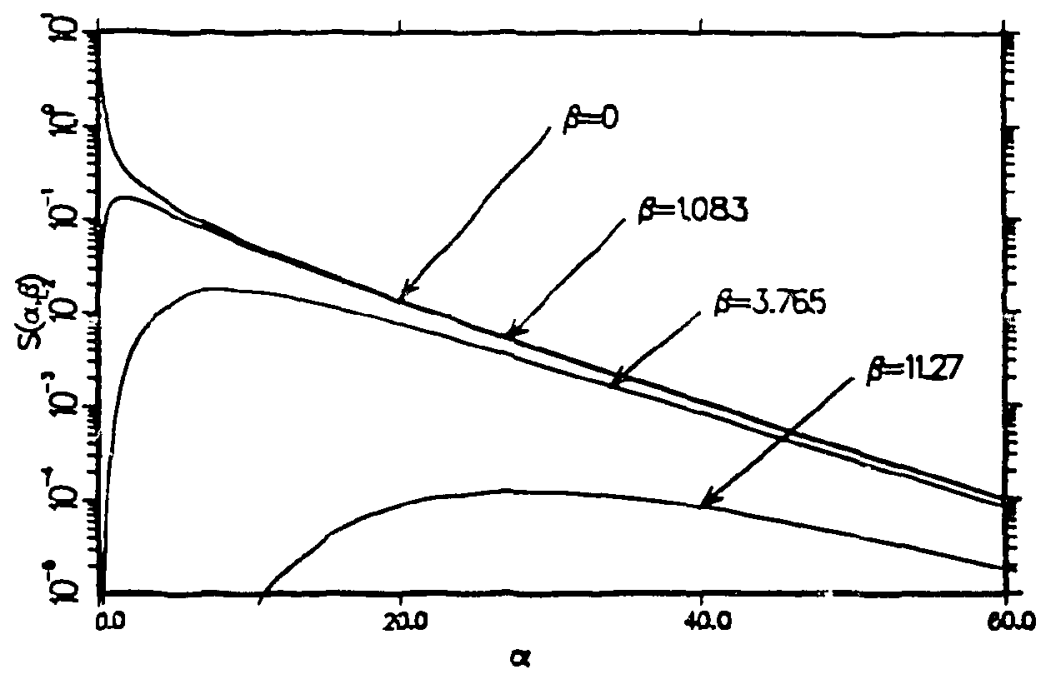

Figure 78: $S(\alpha, \beta)$ curves for liquid methane. Note the diffusive behavior at low $\alpha$ and $\beta$.

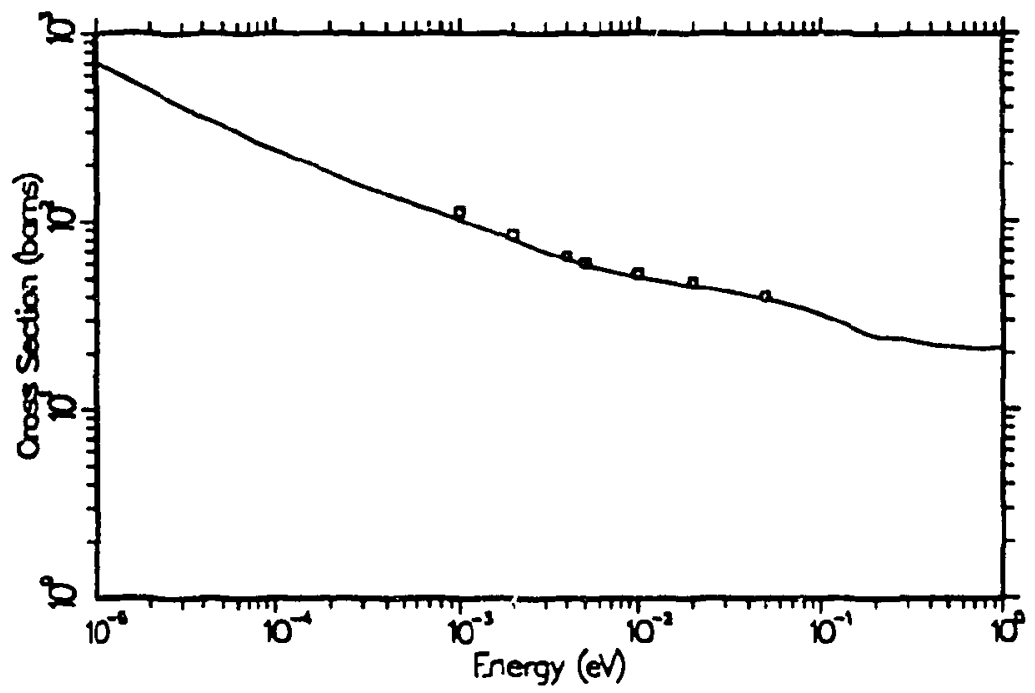

Figure 79: The computed cross section for liquid methane at $100 \mathrm{~K}$ (solid) is cornpared to experimental data (squares) by Whittemore and by Rogalska as quoted by Agrawal and Yip. 


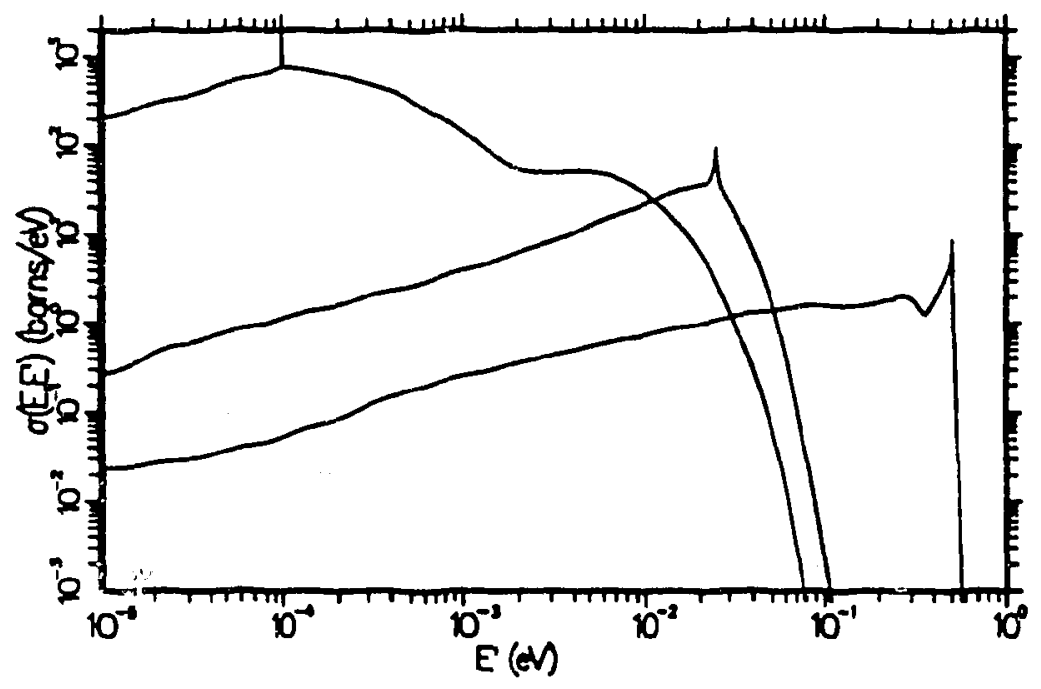

Figure 80: Neutron spectra $\sigma\left(E \rightarrow E^{\prime}\right)$ are shown for $E=.0001, .0253$, and $.503 \mathrm{meV}$. Note the sharp quasj-elastic peak that results from the diffusive term in the theory used here. 


\section{LIQUID HYDROGEN}

As discussed above, we picture a hydrogen molecule bound in a cluster of about 20 molecules and undergoing vibrations similar to those of a hydrogen mclecule in a solid. These clumps then diffuse through the liquid (hindered translations) according to the Egelstaff-Schofield effective width model. This physical situation is described by the Keinert-Sax distribution function (which they kindly provided to us). They assumed a weight of 0.025 for the hindered translation, leaving a valie of 0.475 for the solid-like distribution. In addition, intermolecular coherence is taken into account using the Vineyard approximation. The static structure factor $S(\kappa)$ was provided to us by Keinert and Sax.

This model was then used in LEAPR. The following is the input file used for para hydrogen:

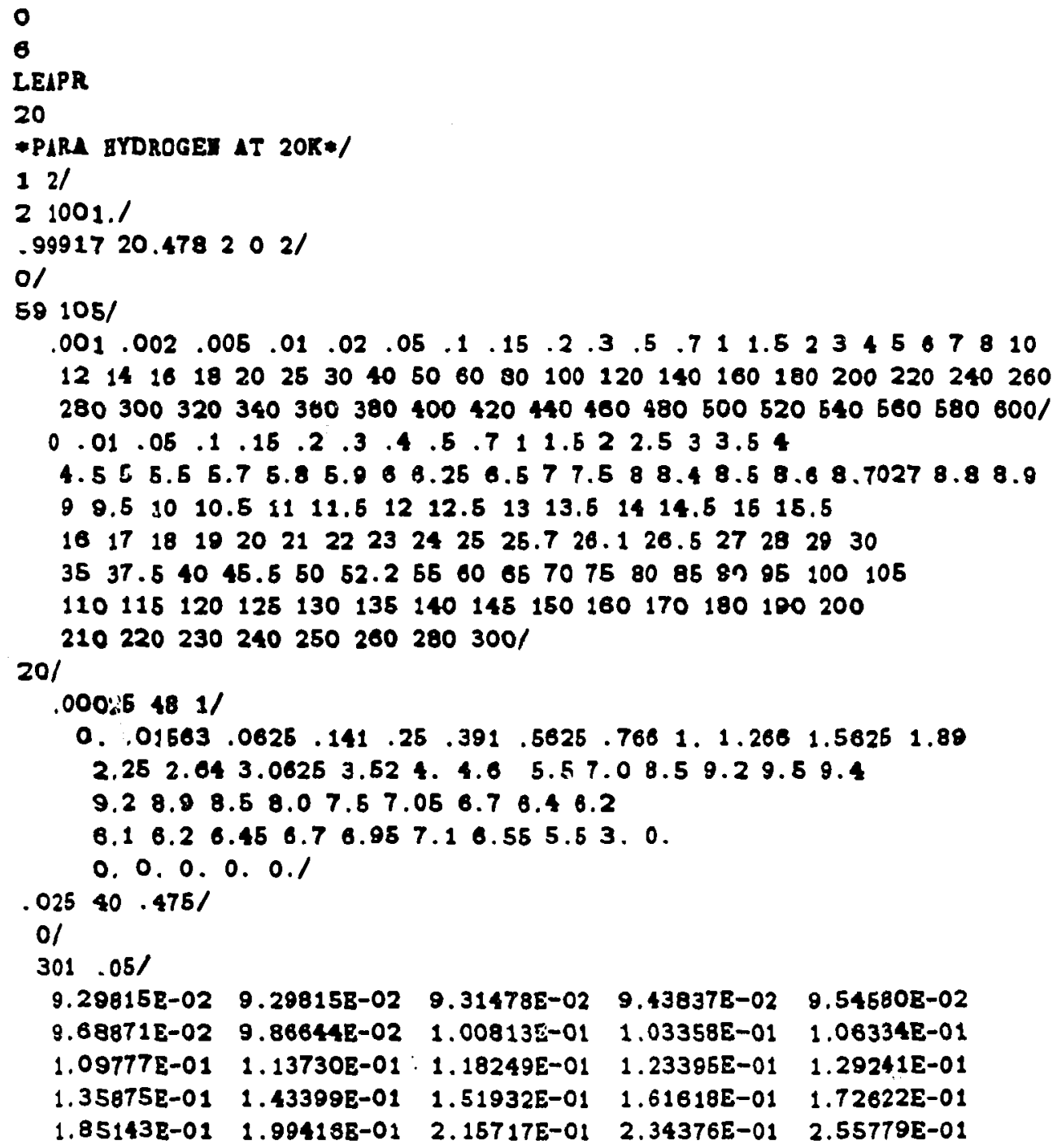




\begin{tabular}{|c|c|c|c|c|}
\hline & & $3 E-01$ & 01 & \\
\hline & & $441 E-01$ & & $98670 E-01$ \\
\hline & $40870 E+00$ & $66330 \varepsilon+00$ & +00 & $.05540 E+00$ \\
\hline $390 \Sigma+00$ & $28030 \mathrm{~B}+00$ & $.33060 \varepsilon+00$ & $.34180 E+00$ & $2.31640 E+00$ \\
\hline $810 E+\infty 0$ & $17080 E+00$ & $05850 E+00$ & $.92640 E+00$ & $1.77900 \mathrm{E}+00$ \\
\hline$:+00$ & $+\infty$ & $D E+\infty O$ & $1.13270 E+00$ & $9.78790 \mathrm{E}-01$ \\
\hline-01 & & .01 & & 20E-01 \\
\hline-01 & & -01 & & $3.28120 \mathrm{E}-01$ \\
\hline-01 & & 01 & & $E-01$ \\
\hline-01 & B-01 & $10 E-01$ & $9.69420 \mathrm{~B}-01$ & $D E+00$ \\
\hline$+\infty$ & & $+\infty 0$ & +00 & $E+00$ \\
\hline & & $\mathrm{B}+\infty$ & $+\infty 0$ & $E+\infty$ \\
\hline & & $+\infty 0$ & +00 & $E+\infty$ \\
\hline+00 & & 00 & +00 & 8-01 \\
\hline & & -01 & & 8-01 \\
\hline & & 01 & & E-01 \\
\hline & & & & \\
\hline & & .01 & & \\
\hline & & +00 & .00 & $+\infty$ \\
\hline$+\infty 0$ & & +00 & 00 & $+\infty$ \\
\hline$+\infty$ & .00 & $+\infty 0$ & -00 & +00 \\
\hline+00 & .00 & +00 & 00 & -01 \\
\hline-01 & -01 & 01 & 01 & -01 \\
\hline-01 & 01 & 01 & 01 & -01 \\
\hline & .01 & 01 & 01 & -01 \\
\hline & -01 & -01 & -01 & $:+00$ \\
\hline .00 & & +00 & 00 & $:+\infty$ \\
\hline .00 & & 00 & & $+\infty$ \\
\hline & & .00 & & $8+\infty$ \\
\hline & & +00 & & +00 \\
\hline & & 01 & & -01 \\
\hline & & 01 & & -01 \\
\hline & & 01 & & -01 \\
\hline & & -01 & -01 & $E-01$ \\
\hline & & $+\infty$ & 100 & $8+\infty$ \\
\hline & & $+\infty 0$ & 100 & $E+\infty$ \\
\hline$+\infty 0$ & & +00 & & $\varepsilon+\infty$ \\
\hline+00 & & 00 & & $+\infty$ \\
\hline & & 01 & & $3-01$ \\
\hline & & -01 & & B-01 \\
\hline & & -01 & & $E-01$ \\
\hline & & -01 & -01 & $E-01$ \\
\hline & & $+\infty 0$ & $+\infty 0$ & $8+00$ \\
\hline & & +00 & $+\infty 0$ & $8+\infty$ \\
\hline & & $+\infty 0$ & $+\infty 0$ & $8+\infty$ \\
\hline & & +00 & $+\infty 0$ & $E+00$ \\
\hline & & 00 & -01 & $3-01$ \\
\hline & & -01 & -01 & $E-01$ \\
\hline & & -01 & & $E-01$ \\
\hline & & & & -01 \\
\hline & & & & $+\infty$ \\
\hline & & $+\infty$ & & $+\infty$ \\
\hline & & $+\infty$ & +00 & $E+\infty 0$ \\
\hline & & 1. & $+\infty 0$ & $E+00$ \\
\hline & & +00 & $90 E+00$ & $1.00010 E+0$ \\
\hline
\end{tabular}




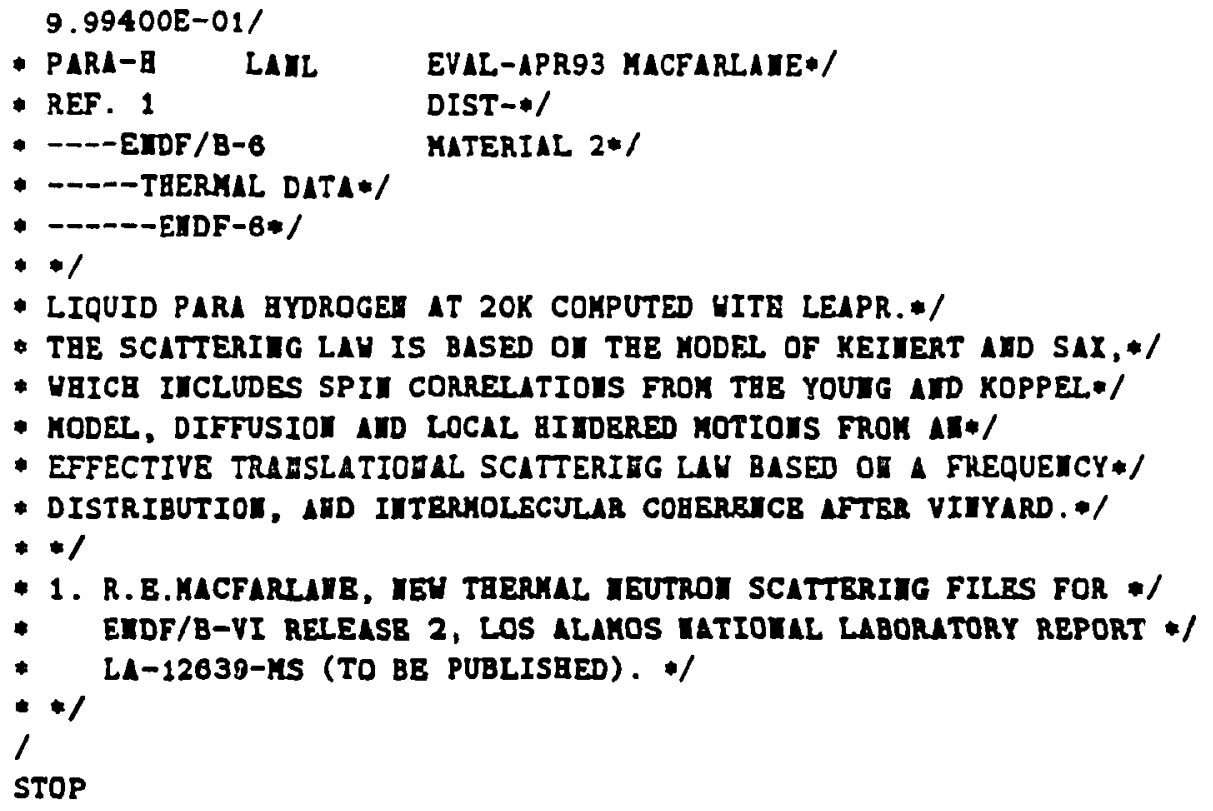

The following is the input used for ortho hydrogen:

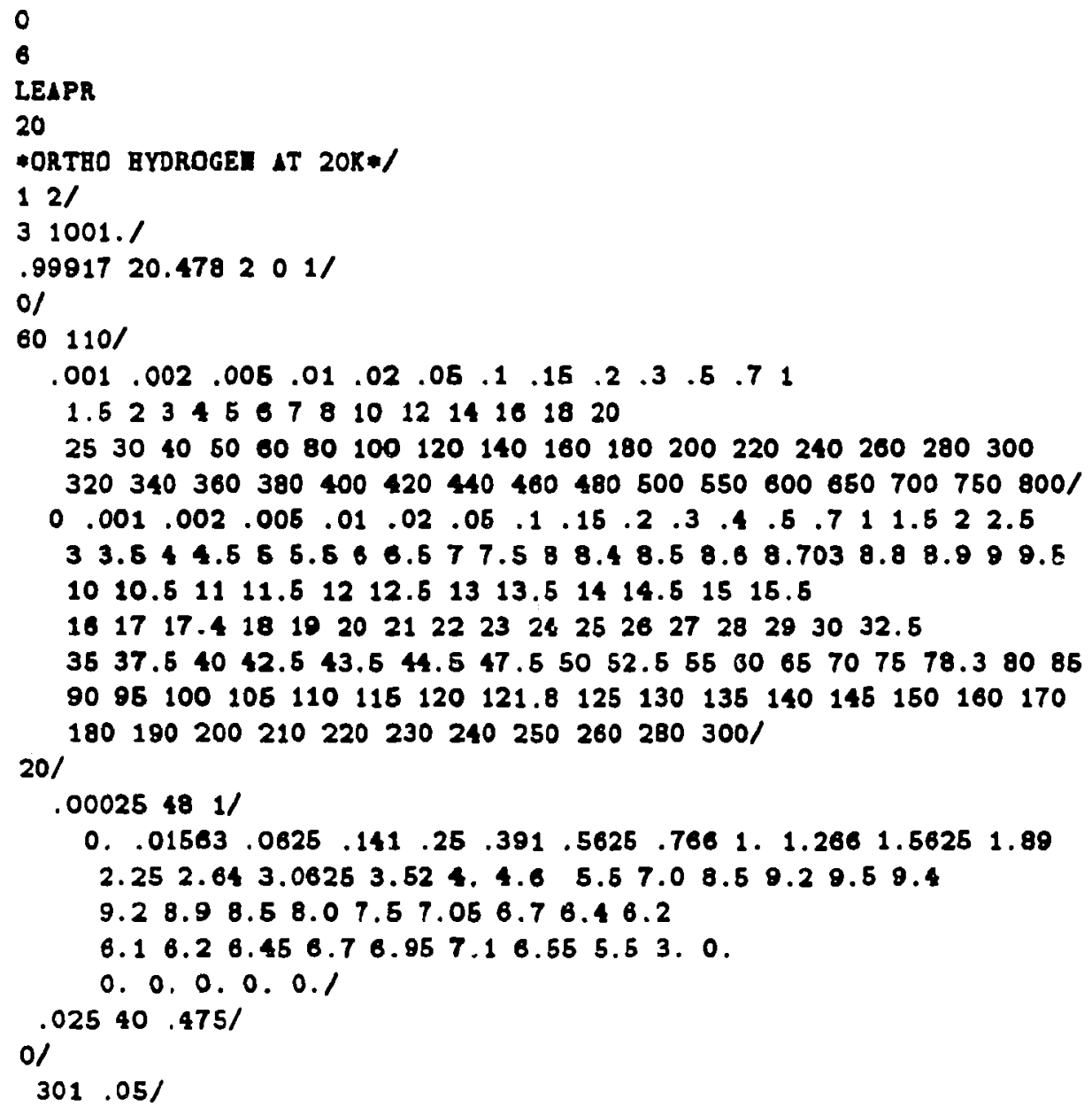




\begin{tabular}{|c|c|c|c|c|}
\hline & & & & - \\
\hline & & $E-01$ & $3 E-01$ & $06334 E-01$ \\
\hline & $3730 E-01$ & & & $29241 E-01$ \\
\hline & $3399 E-01$ & $1932 \varepsilon-01$ & & $.72622 E-01$ \\
\hline & $99416 E-01$ & $15717 \mathrm{E}-01$ & & $.55779 E-01$ \\
\hline $80385 E-01$ & $08731 E-01$ & $41443 E-01$ & & $.22934 E-01$ \\
\hline & & & & \\
\hline & & $E+00$ & & $.05540 E+00$ \\
\hline & & & & $640 E+00$ \\
\hline & & & & \\
\hline & & & & \\
\hline 01 & & & & \\
\hline & & & & \\
\hline OB-01 & & & & \\
\hline $0 \mathrm{E}-01$ & & & & \\
\hline$i+\infty$ & & & & \\
\hline$+\infty$ & & & & \\
\hline & & & & \\
\hline & & & & \\
\hline & & & & \\
\hline & & & & \\
\hline & & & & \\
\hline & & & & \\
\hline & & 00 & .00 & \\
\hline 00 & & -00 & 00 & \\
\hline 00 & & 00 & +00 & \\
\hline 00 & & 00 & 00 & \\
\hline 01 & & 01 & 01 & \\
\hline 01 & & 01 & 01 & \\
\hline 01 & & & & \\
\hline & & & & \\
\hline 00 & & & 00 & \\
\hline 00 & & & & \\
\hline & & & & \\
\hline & & & & \\
\hline & & & & \\
\hline & & -01 & & \\
\hline & & -01 & & \\
\hline 01 & & -01 & & -01 \\
\hline .0 & 1.0 & 100 & +00 & $+\infty 0$ \\
\hline 1.0 & & 100 & +00 & $+\infty$ \\
\hline$+\infty 0$ & & +00 & +00 & $+\infty 0$ \\
\hline 1.0 & & +00 & 00 & $+\infty 0$ \\
\hline 100 & & & & -01 \\
\hline-01 & & -01 & -01 & $3-01$ \\
\hline & & -01 & -01 & -01 \\
\hline .01 & & $z-01$ & -01 & $8-01$ \\
\hline-01 & & $E+\infty$ & +00 & $E+00$ \\
\hline+00 & & +00 & +00 & $z+\infty$ \\
\hline$O E+O O$ & & 100 & $+\infty$ & $E+00$ \\
\hline$\$+\infty$ & $+\infty$ & $+\infty 0$ & $1.00650 E+00$ & $10 E+00$ \\
\hline $40 E+00$ & & $+\infty 0$ & -01 & $E-01$ \\
\hline & & -01 & 9.9 & $e-01$ \\
\hline & & & & -01 \\
\hline $92110 E-01$ & 2740E-01 & $93510 E-01$ & $9.94400 \mathrm{E}-01$ & $.95370 E-01$ \\
\hline
\end{tabular}




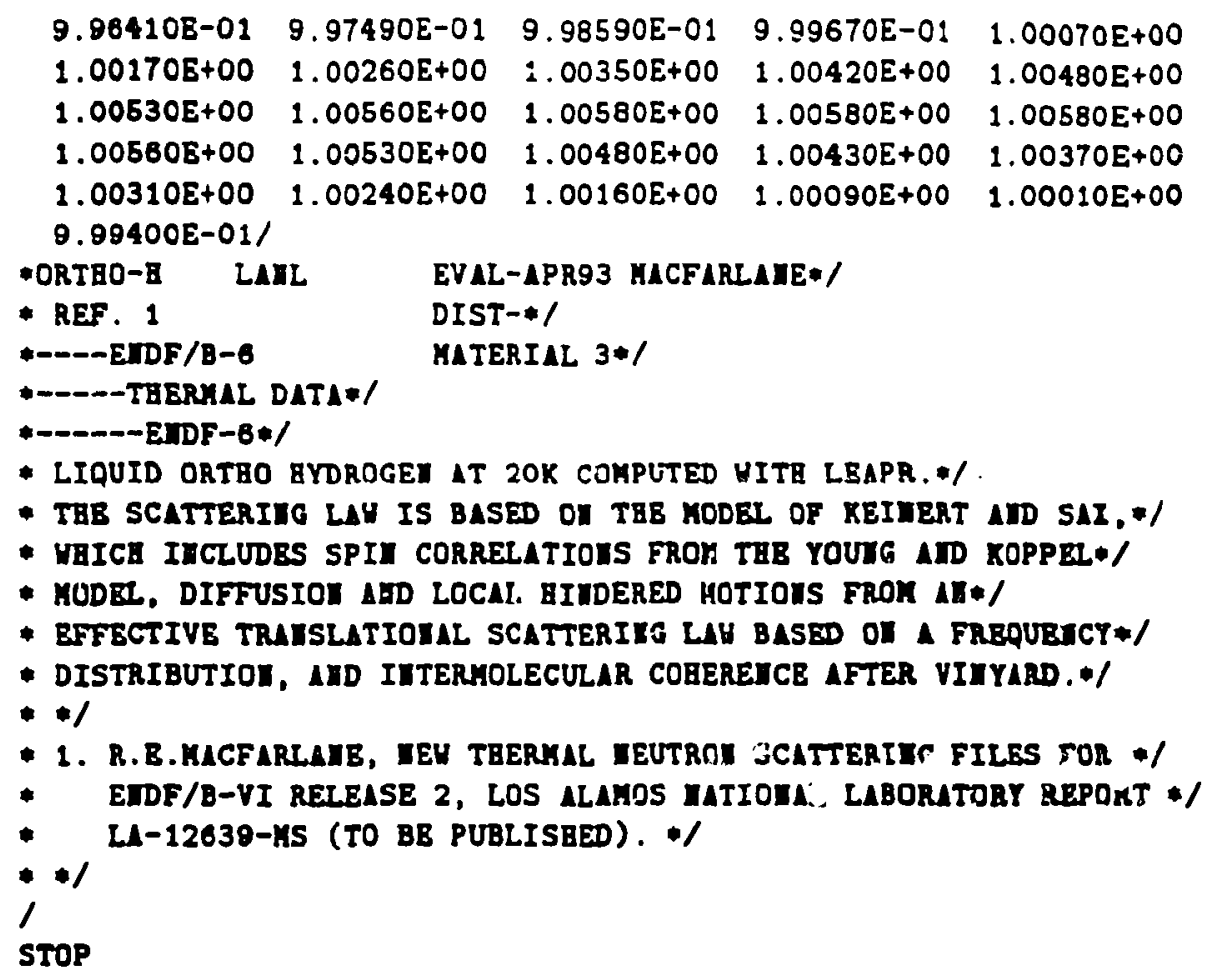

Figures 81 and 82 show the frequency spectrum for the solid-type modes and the static structure factor, respectively.

Several views of the scattering law for liquid para hydrogen are shown in Figures 83 through 86 . The asymmetric version of $S(\alpha, \beta)$ is plotted because liquid hydrogen does not obey the microscopic reversibility condition required for having a symmetric scattering law. Note that the $\alpha$ and $\beta$ grids are sufficiently detailed to represent the important features. The cusps evident in Figure 86 are due to plotting semilog curves on a log-log plot. Similarly, Figures 87 through 90 show the scattering law for liquid ortho hydrogen.

Some examples of cross sections and secondary energy distributions computed by THERMR are shown in Figures 91, 92, and 93. 


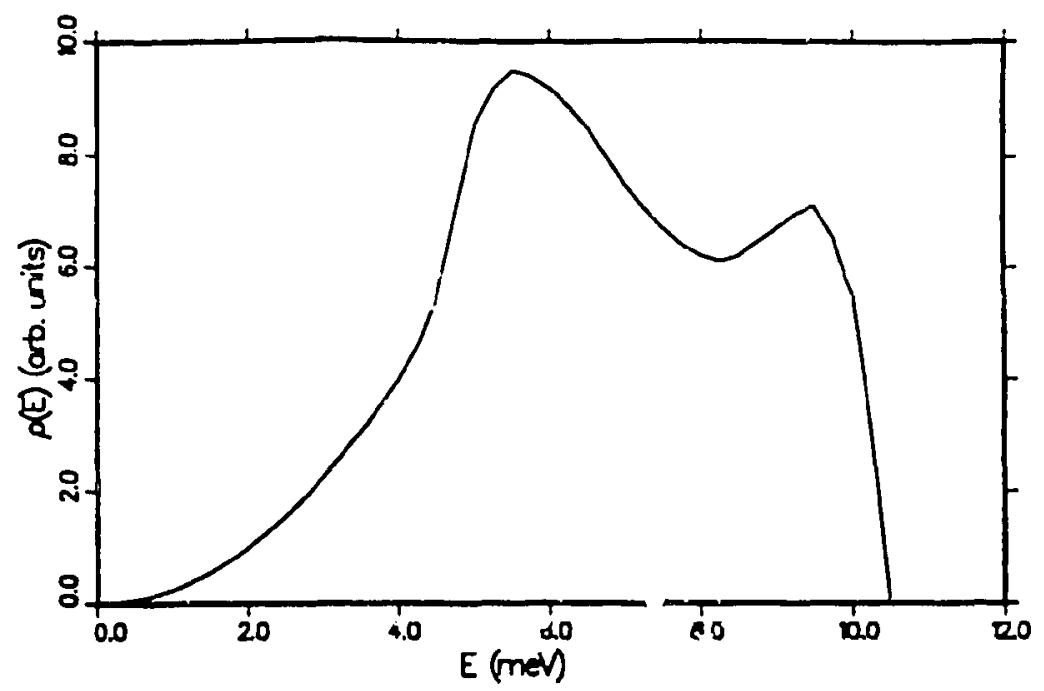

Figure 81: The Keinert-Sax frequency distribution for the effective translational modes of liquid hydrogen.

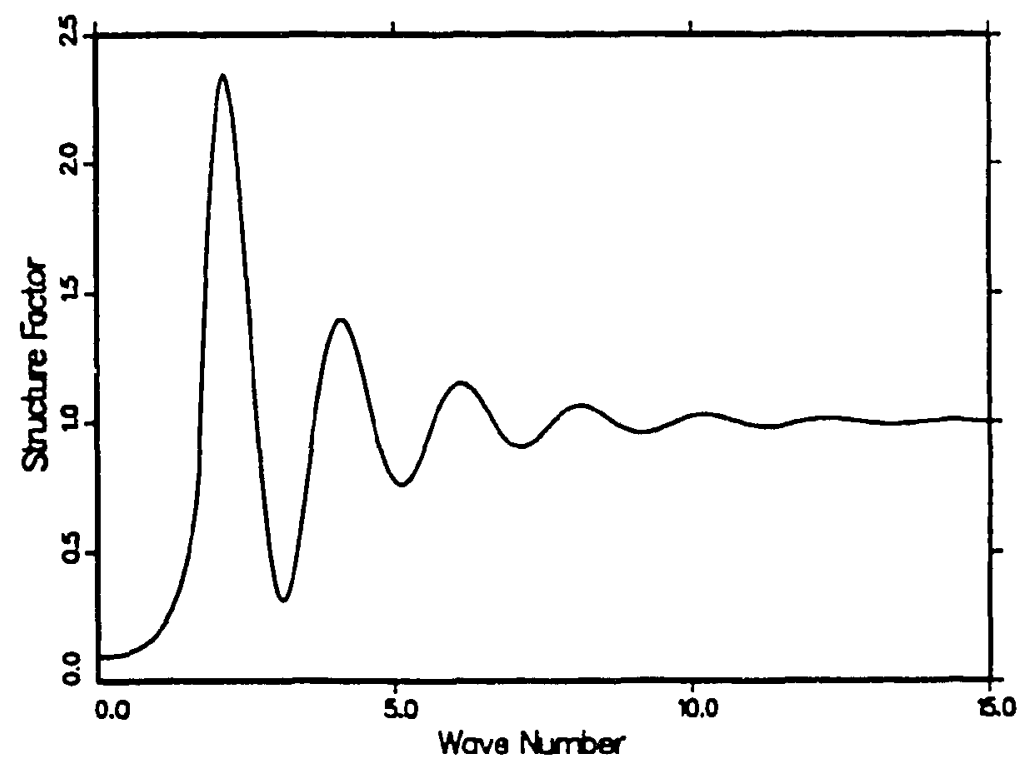

Figure 82: The static structure factor $S(\kappa)$ for liquid hydrogen. 


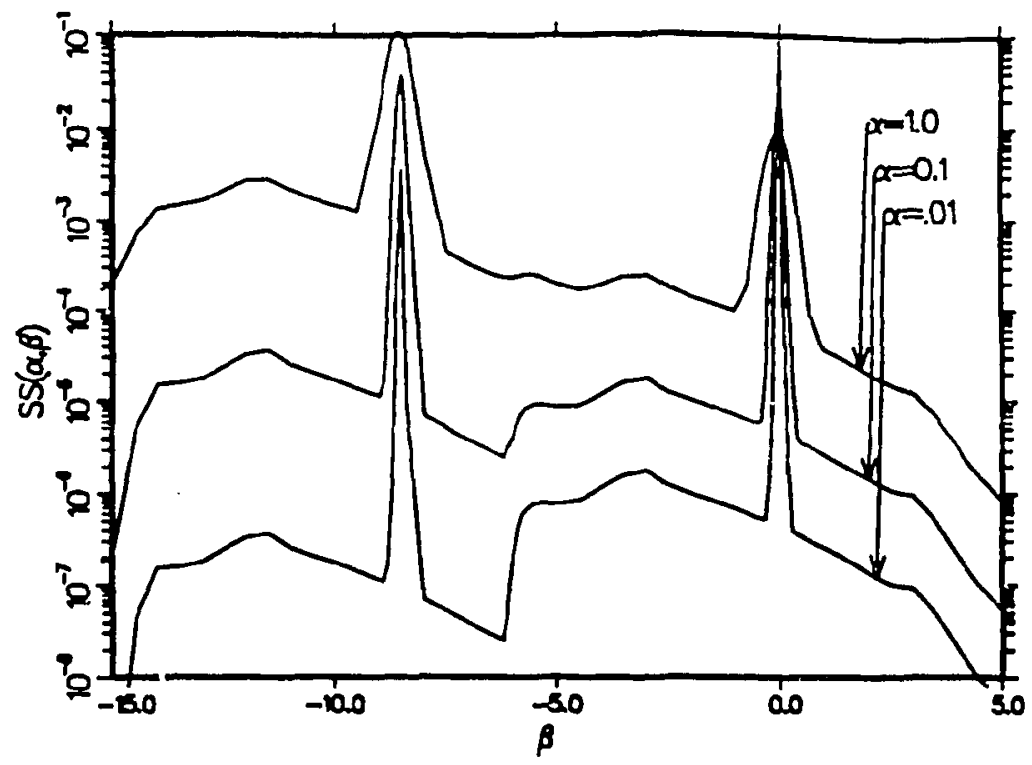

Figure 83: Script-S for liquid para hydrogen at $20 \mathrm{~K}$ is shown as a function of $\beta$ for several $\alpha$ values, concentrating on the low- $\alpha$ and low- $\beta$ range.

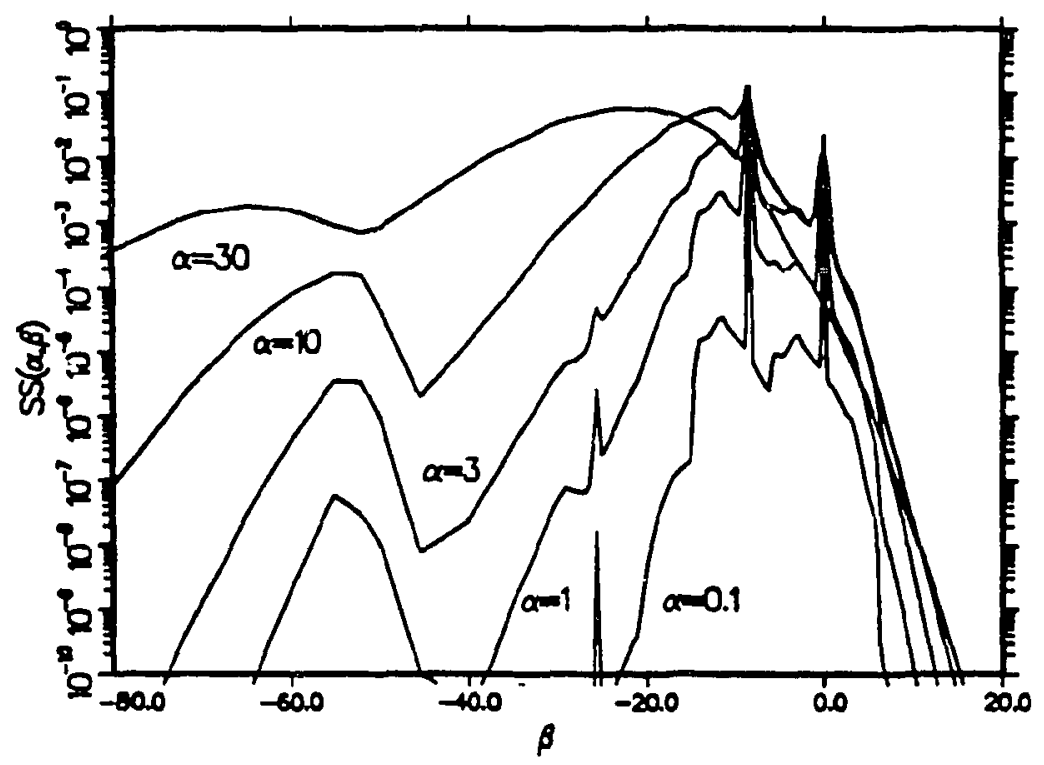

Figure 84: Script-S for liquid para hydrogen at $20 \mathrm{~K}$ is shown as a function of $\beta$ for several $\alpha$ values, concentrating on the middle range of $\alpha$ and $\beta$ values. 


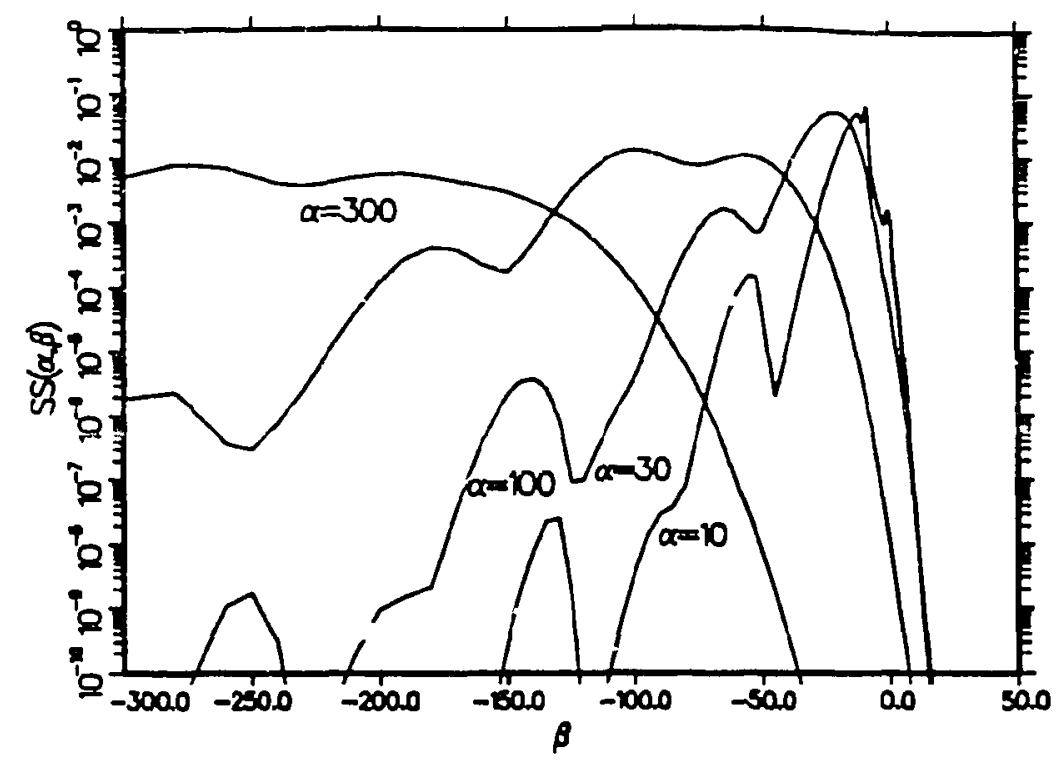

Figure 85: Script-S for liquid para hydrogen at $20 \mathrm{~K}$ is shown as a function of $\beta$ for several $\alpha$ values, concentrating on high $\alpha$ and $\beta$ values.

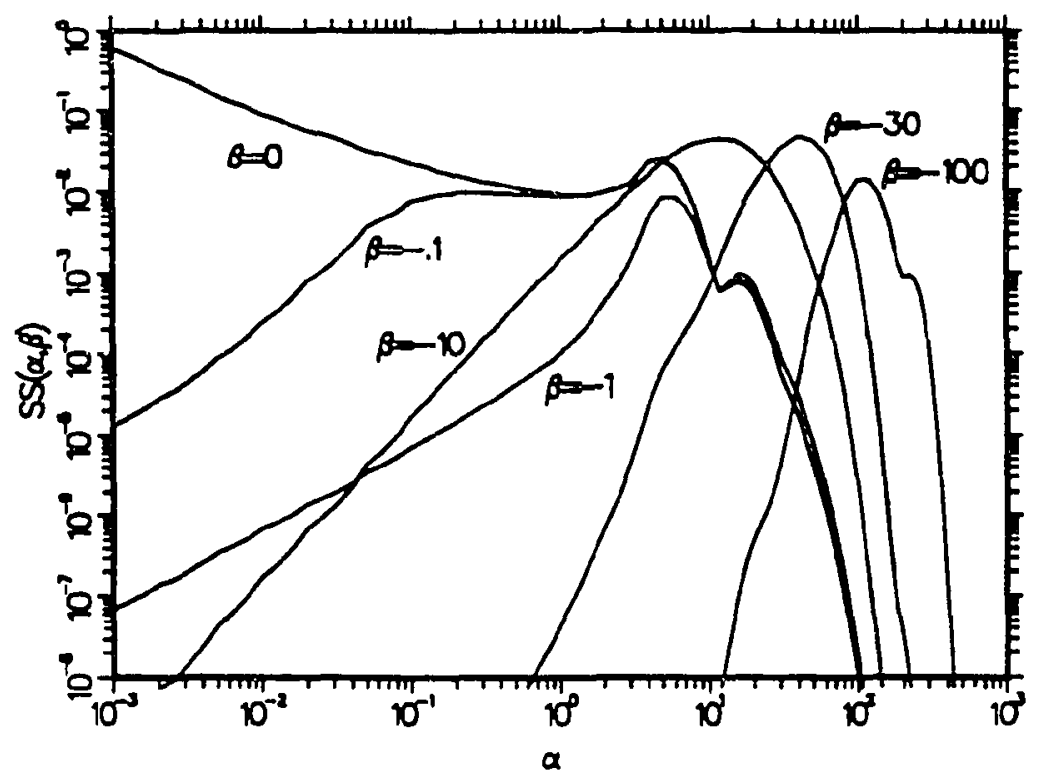

Figure 86: Script-S for liquid para hydrogen at $20 \mathrm{~K}$ is shown as a function of $\alpha$ for several $\beta$ values corresponding to downscatter. 


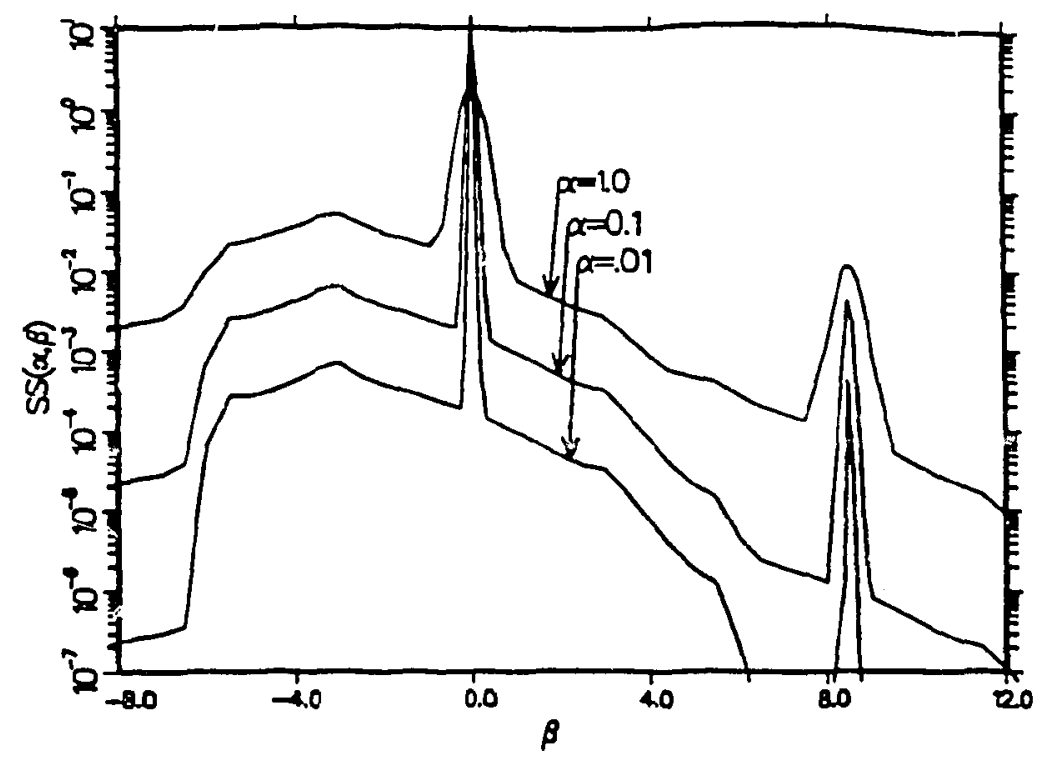

Figure 87: Script-S for liquid ortho hydrogen at $20 \mathrm{~K}$ is shown as a function of $\beta$ for several $\alpha$ values, concentrating on the low- $\alpha$ and low- $\beta$ range.

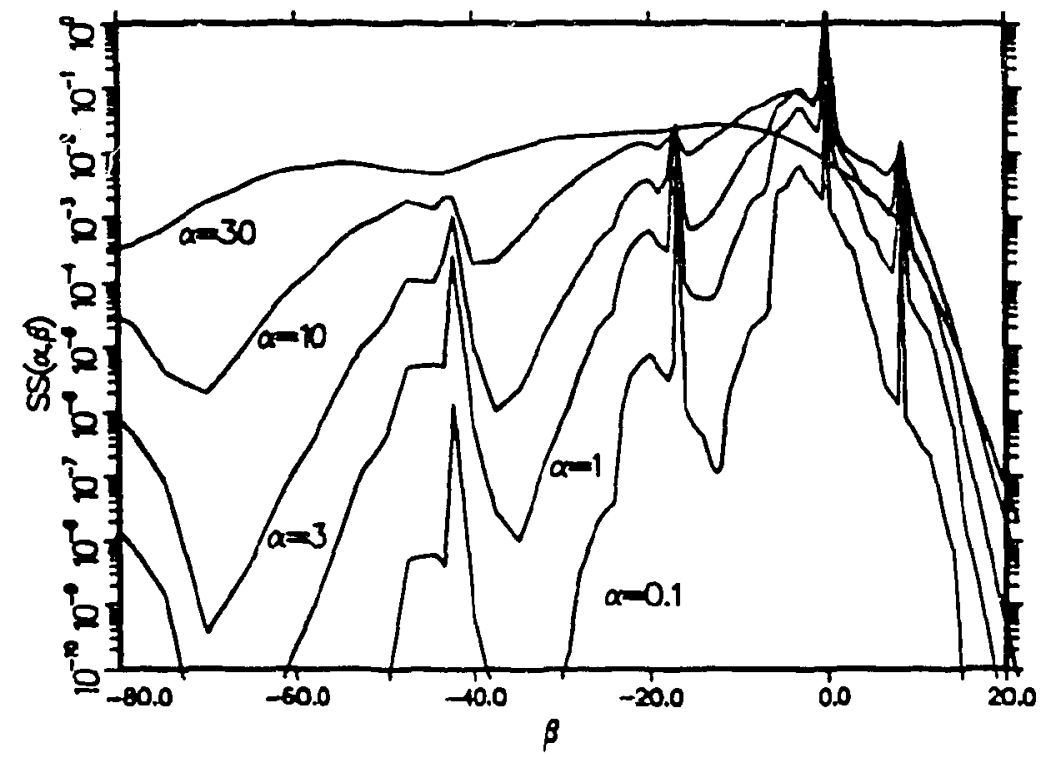

Figure 88: Script-S for liquid ortho hydrogen at $20 \mathrm{~K}$ is shown as a function of $\beta$ for several $\alpha$ values, concentrating on the middle range of $\alpha$ and $\beta$ values. 


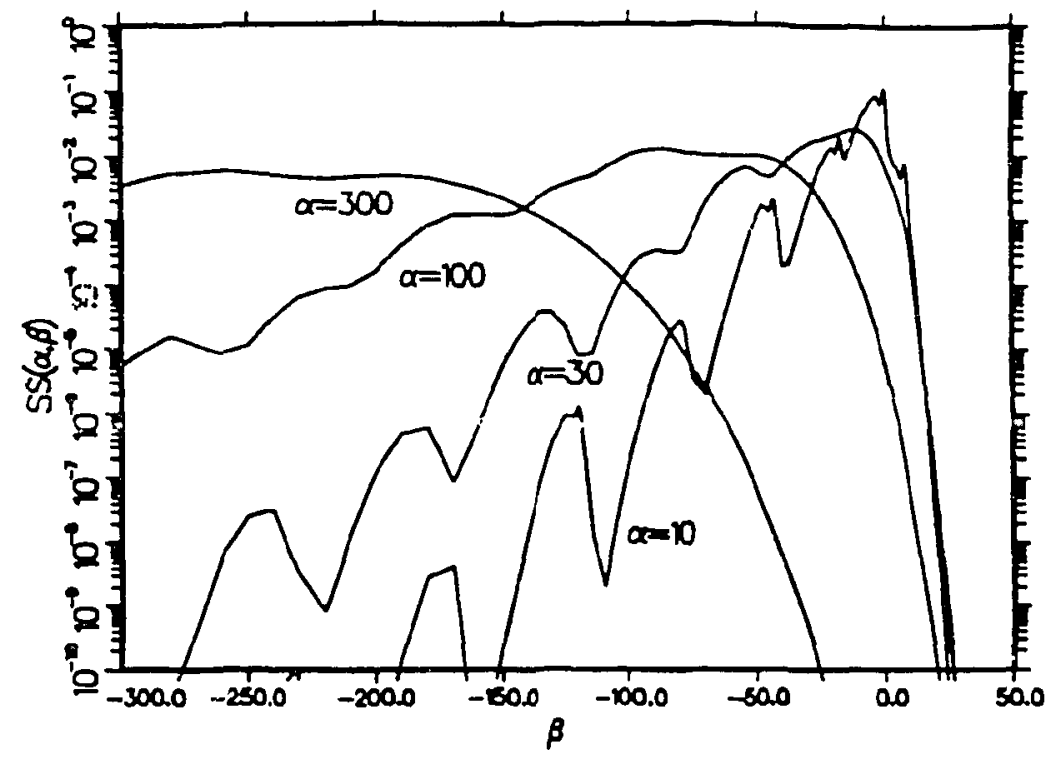

Figure 89: Script-S for liquid ortho hydrogen at $20 \mathrm{~K}$ is shown as a function of $\beta$ for several $\alpha$ values, concentrating o"1 high $\alpha$ and $\beta$ values.

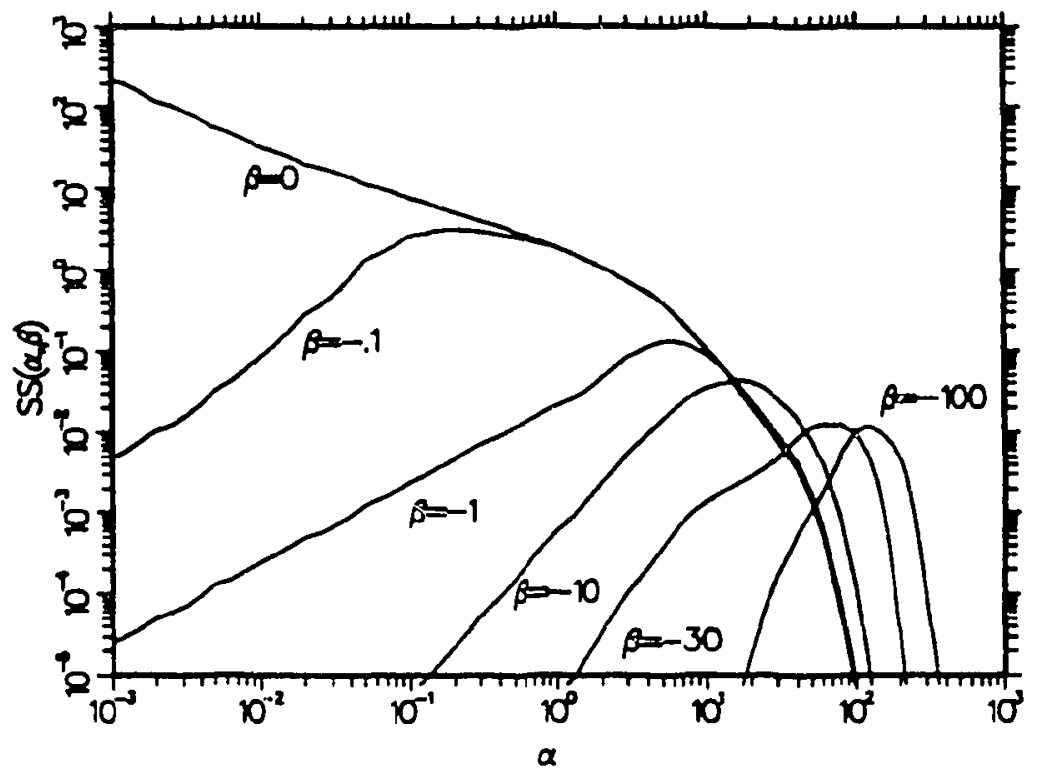

Figure 90: Script-S for liquid ortho hydrogen at $20 \mathrm{~K}$ is shown as a function of $\alpha$ for several $\beta$ values corresponding to downscatter. 


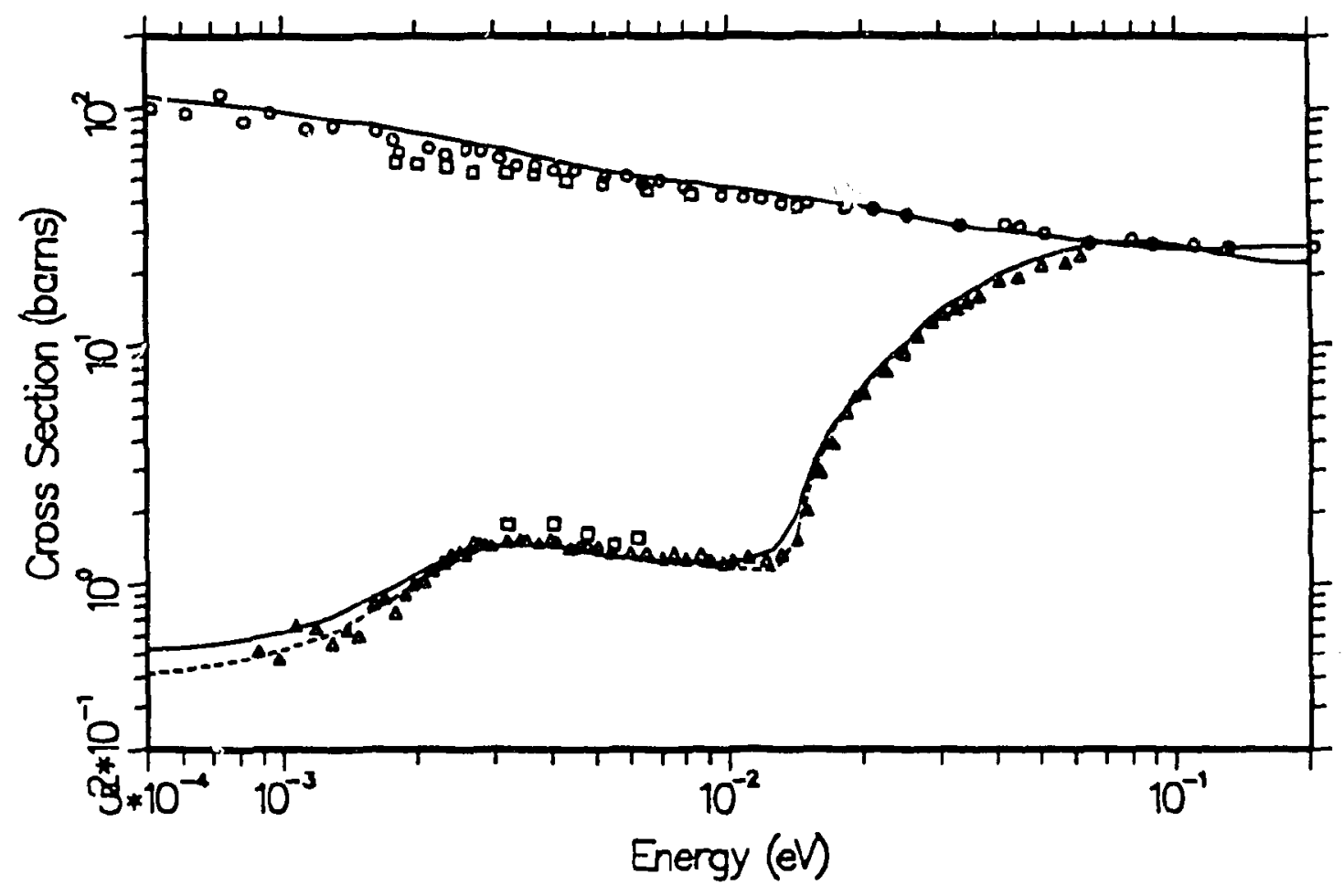

Figure 91: The cross sections for liquid ortho hydrogen (upper curve) and liquid para hydrogen (lower curve) are compared with experimental data (see Ref. 29) obtained by Squires (gas) at $20 \mathrm{~K}$ (squares), Whittemore at $20 \mathrm{~K}$ (circles), and Seiffert at $14 \mathrm{~K}$ (triangles). The solid curves are at $20 \mathrm{~K}$, and the dashed curve is at $14 \mathrm{~K}$. The sharp drop in the para cross section below $0.05 \mathrm{eV}$ is due to spin coherence, and the second drop below $.003 \mathrm{eV}$ is due to intermolecular interference. 


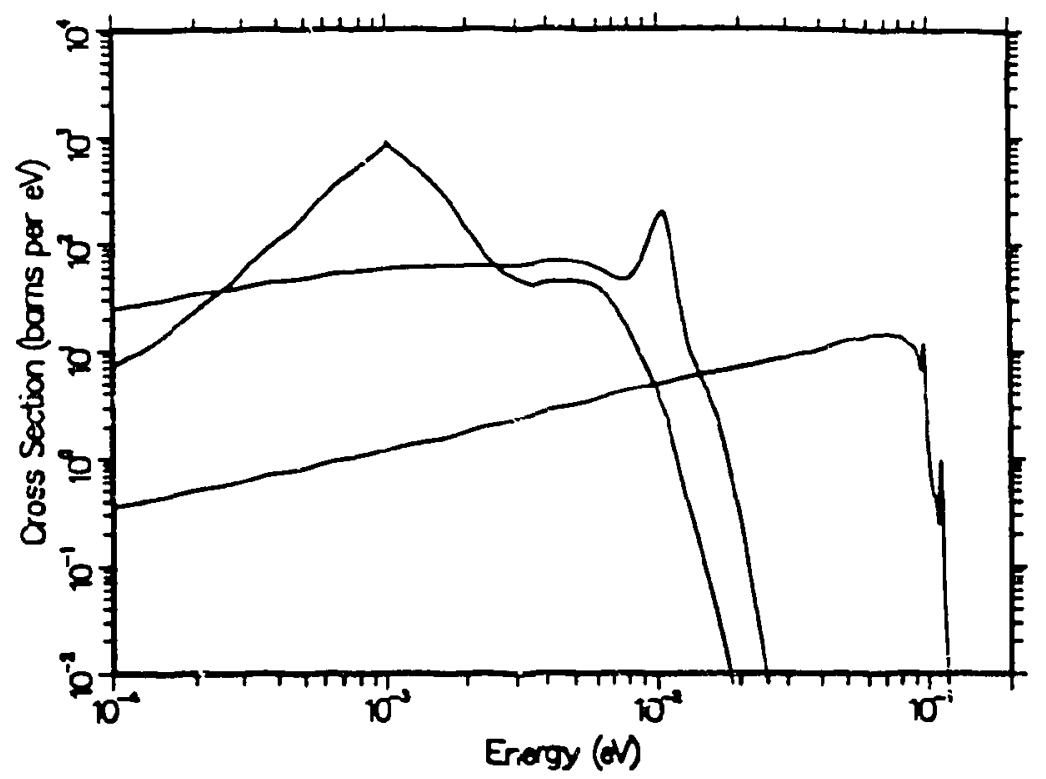

Figure 92: The spectra $\sigma\left(E \rightarrow E^{\prime}\right)$ for liquid para hydrogen are shown for $E=.001, .0106$, and $.112 \mathrm{eV}$.

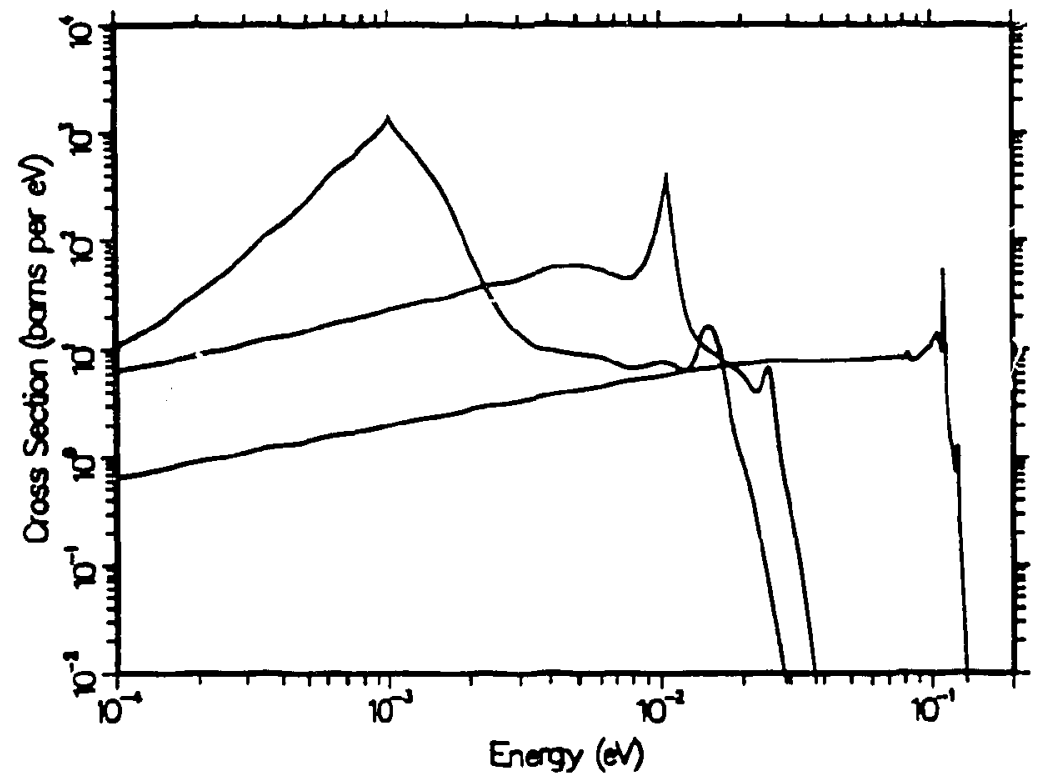

Figure 93: The spectra $\sigma\left(E \rightarrow E^{\prime}\right)$ for liquid ortho hydrogen are shown for $E=.001, .0106$, and $.112 \mathrm{eV}$. 


\section{LIQUID DEUTERIUM}

The model for liquid $\mathrm{D}_{2}$ is almost identical to the one described above for liquid $\mathrm{H}_{2}$. The diffusion parameters used for liquid deuterium are identical to those used for liquid hydrogen. This means that we are assuming that about 10 deuterium molecules are ccontained in the clump that is diffusing through the liquid. The static structure factor used to account for intermolecular interference was kindly provided to us by Keinert and Sax.

The LEAPR input file for para deuterium follows:

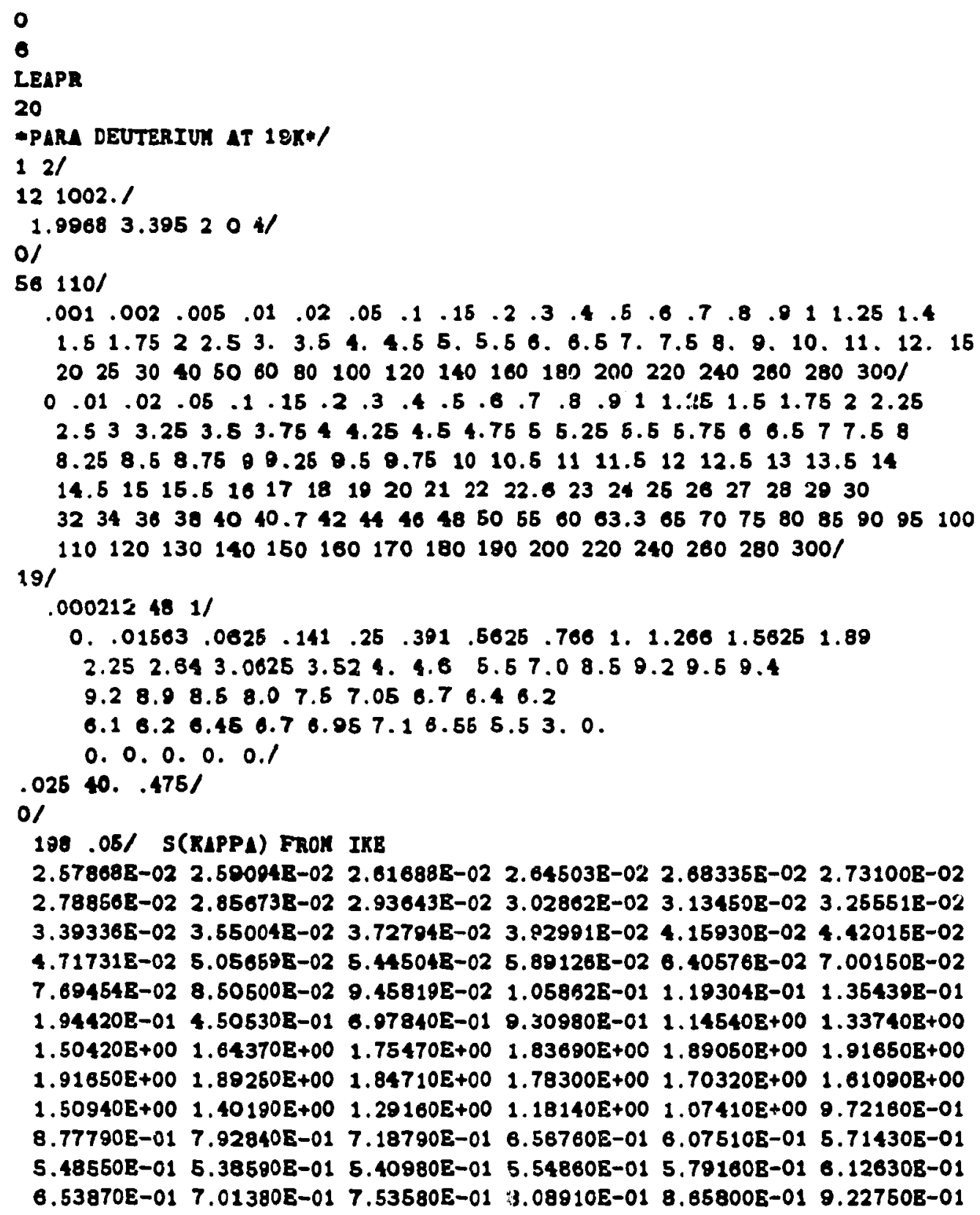


9.78350E-01 $1.03130 E+001.08050 E+00 \quad 1.12490 E+00 \quad 1.16360 E+001.19620 E+00$ 1.22200E+00 1.24100E+00 1.25290E+00 1.25790E+00 $1.25620 E+00 \quad 1.24830 E+00$ 1. $23470 E+001.21600 E+001.19300 E+00 \quad 1.16650 E+001.13730 E+001.10620 E+00$ $1.07410 E+001.04190 E+001.01030 E+00$ 9.80050E-01 9.51840E-01 9.26230E-01 9.037OOE-O 2 8.84650E-01 8.69350E-01 8.57990E-01 8.5063CE-01 8.47250E-01 8.47730E-O1 E.51870E-0i 8.59390E-01 8.68930E-01 8.83090E-01 8.98440E-01 9.15500E-01 9.33770E-01 9.52760E-01 9.71980E-01 9.90970E-01 $1.00930 \mathrm{E}+00$ $1.02650 \mathrm{E}+00 \quad 1.04230 \mathrm{E}+00 \quad 1.05630 \mathrm{E}+00 \quad 1.08840 \mathrm{E}+00 \quad 1.07820 \mathrm{E}+00 \quad 1.08580 \mathrm{E}+00$ $1.09090 E+00 \quad 1.09370 E+00 \quad 1.09410 E+00 \quad 1.09230 E+00 \quad 1.08830 E+001.08250 E+00$ $1.07490 \mathrm{E}+001.06590 \mathrm{E}+001.05580 \mathrm{E}+00 \quad 1.04480 \mathrm{E}+001 . \mathrm{C} 3330 \mathrm{E}+001.02140 \mathrm{E}+00$

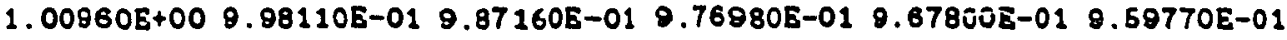
9.53030E-01 $9.47680 \mathrm{~B}-01 \quad 9.43800 \mathrm{~B}-01 \quad 9.41390 \mathrm{~B}-01 \quad 9.40460 \mathrm{E}-01 \quad 9.40950 \mathrm{E}-01$ 0.42800E-01 $9.46900 \mathrm{E}-01 \quad 0.50130 \mathrm{E}-01 \quad 9.65330 \mathrm{E}-01 \quad 0.61360 \mathrm{~B}-01 \quad 9.68020 \mathrm{E}-01$ 9.751408-01 9.82530E-01 $9.90020 \mathrm{E}-01 \quad 9.97420 \mathrm{E}-01 \quad 1.00460 \mathrm{E}+001.01130 \mathrm{E}+00$ $1.01750 \mathrm{E}+00 \quad 1.02300 \mathrm{~B}+00 \quad 1.02780 \mathrm{~B}+00 \quad 2.03170 \mathrm{~B}+00 \quad 1.03480 \mathrm{E}+00 \quad 1.03600 \mathrm{~B}+00$ $1.03810 E+001.03830 \mathrm{~B}+001.03770 \mathrm{E}+00 \quad 1.03620 \mathrm{~B}+001.03390 \mathrm{~B}+001.03100 \mathrm{~B}+00$ $1.02750 \mathrm{E}+00 \quad 1.02350 \mathrm{E}+001.01910 \mathrm{E}+00 \quad 1.01440 \mathrm{E}+00 \quad 1.00970 \mathrm{E}+001.00480 \mathrm{E}+00$ 1. $00010 E+009.95590 \mathrm{~B}-01 \quad 9.91330 \mathrm{E}-01 \quad 9.87450 \mathrm{~B}-01 \quad 9.84010 \mathrm{~B}-01 \quad 9.81070 \mathrm{E}-01$ $9.78670 \mathrm{~B}-019.76860 \mathrm{~B}-01 \quad 9.75640 \mathrm{~B}-01 \quad 9.75030 \mathrm{~B}-01 \quad 0.75000 \mathrm{~B}-01 \quad 9.75540 \mathrm{~B}-01$ 9.7661OB-01 9.78170B-01 9.801608-01 9.82520B-01 9.86280B-01 9.88060B-01 $9.91110 \mathrm{E}-019.94230 \mathrm{E}-01 \quad 9.97330 \mathrm{~B}-01 \quad 1.00040 \mathrm{E}+001.00340 \mathrm{~B}+001.00610 \mathrm{E}+00 /$ - PARA-D LAIR EVAL-APR93 MICFARLAIE */

The LEAPR input file for liquid ortho deuterium follows:

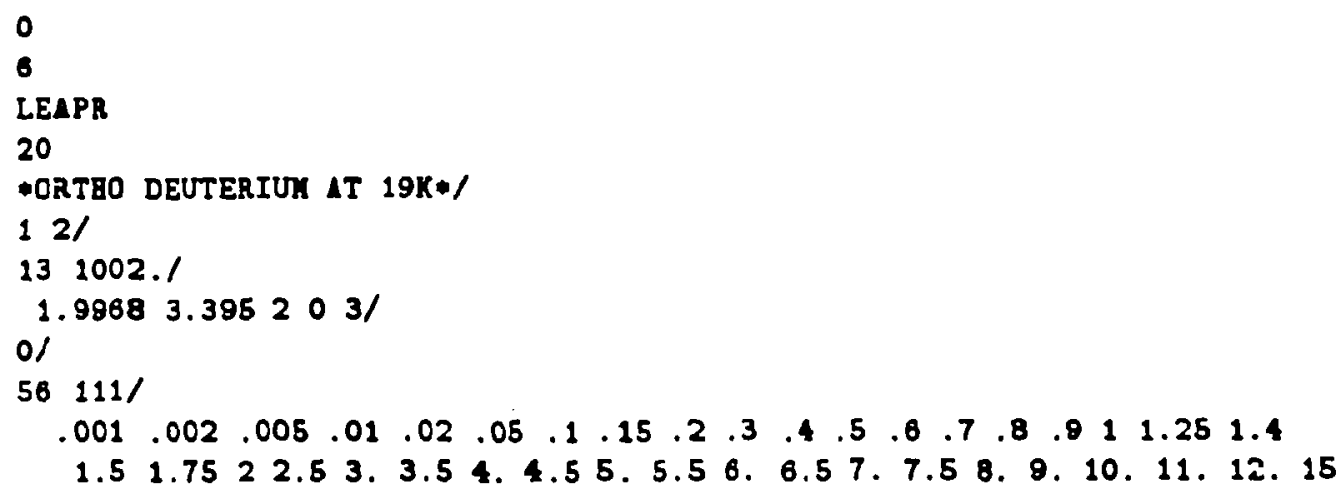


$2026 \quad 30 \quad 4050 \quad 60 \quad 80 \quad 100 \quad 120 \quad 140 \quad 160 \quad 180200220240 \quad 260280 \quad 300 /$ $\begin{array}{lllllllllllllllllllll}0.01 & .02 & .05 & .1 & .15 & .2 & .3 & .4 & .5 & .6 & .7 & .8 & .9 & 1.25 & 1.5 & 1.75 & 2 & 2.25 & 2.5\end{array}$ $\begin{array}{lllllllllllllllllllllll}2.75 & 3 & 3.25 & 3.5 & 3.75 & 4 & 4.25 & 4.5 & 4.75 & 5 & 5.25 & 5.5 & 5.75 & 6 & 6.5 & 7 & 7.5 & 8\end{array}$ $\begin{array}{llllllllllllllll}8.5 & 9.5 & 10 & 10.5 & 11 & 11.5 & 12 & 12.5 & 13 & 13.25 & 13.6 & 13.75 & 14 & 14.5\end{array}$ $\begin{array}{llllllllllllllllllll}15 & 15.5 & 6 & 17 & 18 & 18 & 20 & 21 & 22 & 23 & 24 & 25 & 26 & 26.5 & 27 & 27.5 & 28 & 29 & 30 & 31.6\end{array}$ $\begin{array}{lllllllllllllllllllllllll}32 & 34 & 36 & 38 & 40 & 42 & 44 & 45.2 & 46 & 48 & 50 & 55 & 60 & 6.3 .65 & 67.8 & 70 & 75 & 80 & 85 & 90 & 95 & 100\end{array}$ $\begin{array}{llllllllllllllllll}110 & 120 & 130 & 140 & : 50 & 160 & 170 & 180 & 190 & 200 & 220 & 240 & 260 & 280 & 300 /\end{array}$ $19 /$

$.000212481 /$

$\begin{array}{lllllllllllll}0.01563 & .0625 & .141 & .25 & .391 & .5625 & .766 & 1 . & 1.266 & 1.5625 & 1.89\end{array}$

$2.252 .643 .0625 \quad 3.524 .4 .6 \quad 5.5 \quad 7.0 \quad 8.5 \quad 9.29 .5 \quad 9.4$

$9.28 .98 .5 \quad 8.0 \quad 7.5 \quad 7.05 \quad 6.7 \quad 6.46 .2$

$6.16 .26 .456 .7 \quad 0.957 .16 .555 .5 \quad 3.0$.

0. 0.0 .0 .0 .1

$.02540 . .475 /$

$0 /$

$198.05 /$ S(KLPPL) FROK IKE

2.57868B-02 2.59094E-02 2.61688E-02 2.645038-02 2.683358-02 2.731008-02

2.78856E-02 2.85673E-02 2.93643E-02 3.02862B-02 3.13450B-02 3.25551B-G2

3. 39336I-02 3.55004E-02 3.72794B-02 3.92991B-02 \&.15930B-02 \&.42015E-02

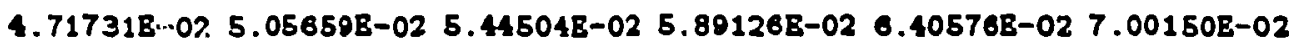

7.69454E-02 8.50500E-02 9.45819E-02 $1.05862 E-01 \quad 1.19304 E-01 \quad 1.35439 B-01$

$1.94420 \mathrm{~B}-01 \quad \mathrm{C} .50530 \mathrm{E}-01 \quad 6.97840 \mathrm{E}-01 \quad 9.30980 \mathrm{~B}-01 \quad 1.14540 \mathrm{~B}+001.33740 \mathrm{~B}+00$

$1.50420 \mathrm{~B}+00 \quad 1.64370 \mathrm{E}+00 \quad 1.75470 \mathrm{E}+00 \quad 1.83690 \mathrm{~B}+00 \quad 1.8905 \mathrm{JE}+001.91650 \mathrm{E}+00$

$1.91650 \mathrm{~B} \div 00 \quad 1.89250 \mathrm{~B}+001.84710 \mathrm{E}+001.78300 \mathrm{~B}+001.70320 \mathrm{~B}+001.61090 \mathrm{~B}+00$

$1.50940 \mathrm{E}+001.40180 \mathrm{E}+00 \quad 1.29160 \mathrm{E}+00 \quad 1.18140 \mathrm{E}+00 \quad 1.07410 \mathrm{~B}+009.72160 \mathrm{~B}-01$

8.777.9OB-01 7.92840E-01 7.18790E-01 6.56760B-01 6.07510B-01 5.71430B-01

5.4855OB-01 5.38590B-01 5.40880B-01 5.64860E-01 5.79160B-01 6.12630B-01

6.53870B-01 7.01380B-01 7.53580B-01 8.08910E-01 8.65800B-01 9.22750B-01

9.78350B-01 $1.03130 \mathrm{~B}+00 \quad 1.08050 \mathrm{~B}+00 \quad 1.12490 \mathrm{~B}+00 \quad 1.16360 \mathrm{~B}+001.18620 \mathrm{~B}+00$

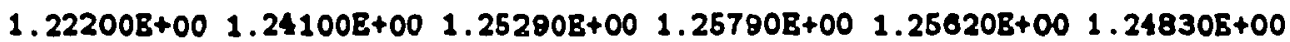

$1.23470 \mathrm{~B}+00 \quad 1.21600 \mathrm{E}+00 \quad 1.15300 \mathrm{~B}+00 \quad 1.16650 \mathrm{~B}+00 \quad 1.13730 \mathrm{~B}+00 \quad 1.10620 \mathrm{~B}+00$

$1.07410 \mathrm{~B}+00 \quad 1.04190 \mathrm{E}+00 \quad 1.01030 \mathrm{E}+00 \quad 9.80050 \mathrm{~B}-01 \quad 0.51840 \mathrm{~B}-01 \quad 0.26230 \mathrm{~B}-01$

9.03700B-01 8.84650B-01 8.69350B-01 8.57990E-01 8.50630B-01 8. $7250 \mathrm{~B}-01$

8.47730B-01 8.51870B-01 8.59390B-01 8.69930B-01 8.83000B-01 8.98440B-01

9.15500E-01 9.3377OE-01 9.62760E-01 9.71980E-01 $9.90970 \mathrm{~B}-011.00930 \mathrm{~B}+00$

$1.02650 \mathrm{~B}+001.04230 \mathrm{E}+001.05630 \mathrm{E}+001.06840 \mathrm{E}+001.07820 \mathrm{~B}+001.08580 \mathrm{~B}+00$

$1.09090 \mathrm{~B}+001.09370 \mathrm{~B}+00 \quad 1.09410 \mathrm{E}+00 \quad 1.09230 \mathrm{E}+00 \quad 1.08830 \mathrm{~B}+001.08250 \mathrm{~B}+00$

$1.07400 \mathrm{~B}+001.06590 \mathrm{~B}+00 \quad 1.05580 \mathrm{~B}+00 \quad 1.04480 \mathrm{E}+00 \quad 1.03330 \mathrm{~B}+001.02140 \mathrm{E}+00$

$1.00960 \mathrm{~B}+00 \quad 9.98110 \mathrm{~B}-01 \quad 9.87160 \mathrm{~B}-01 \quad 9.76980 \mathrm{~B}-01 \quad 9.67800 \mathrm{~B}-01 \quad 9.69770 \mathrm{~B}-01$

9.53030B-01 $9.47600 \mathrm{~B}-01 \quad 9.43800 \mathrm{~B}-01 \quad 9.41390 \mathrm{~B}-01 \quad 9.40460 \mathrm{~B}-01 \quad 9.40950 \mathrm{~B}-01$

9.42E00E-01 9.45000B-01 9.50130B-01 9.55330E-01 9.61360B-01 9.68020B-01

9.75140B-01 9.82530B-01 9.90020B-01 9.97420B-01 $1.00460 \mathrm{~B}+001.01130 \mathrm{E}+00$

$\therefore .01750 \mathrm{~B}+001.02300 \mathrm{E}+001.02780 \mathrm{E}+001.03170 \mathrm{E}+001.03480 \mathrm{E}+001.03690 \mathrm{E}+00$

$1.03810 B+001.03830 B+001.03770 E+001.03620 E+001.03390 E+001.03100 E+00$

$1.02750 \mathrm{~B}+001.02350 \mathrm{E}+00 \quad 1.01910 \mathrm{E}+00 \quad 1.01440 \mathrm{E}+00 \quad 1.00970 \mathrm{~B}+001.00480 \mathrm{~B}+00$

1.00010E+00 9.95590E-01 9.91330E-01 9.87460E-01 9.84010E-01 9.81070E-01

9.7867OE-01 9.76860E-01 9.7564OE-01 9.75030E-01 9.7500OB-01 9.75E4OB-01

9.76610E-01 9.78170E-01 9.80160E-01 9.82520E-01 9.85180B-01 9.88060E-01

$9.91110 E-019.94230 \mathrm{E}-01 \quad 9.97360 \mathrm{E}-01 \quad 1.00040 \mathrm{E}+00 \quad 1.003408+001.00610 \mathrm{E}+00 /$

* ORTEO-D LAIL EVAL-APR93 MLCFARLAEB */

* REF. 1 DIST- */

* - - ETDF/B-6 HATERIAL $13 * /$

- - - - THERMAL DATA*

- - - - - EIDF-6 
- liquid ortho devteriut at 19K computed hitg leapr. *

- tge scatterigg lay is based ol the model of keidest and sax, */

- HHICB IICLUDES SPIY CORRELATIOUS FROM THE YOUIG IID KOPPEL *

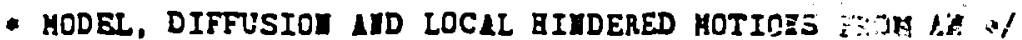

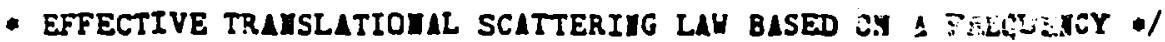

- distributiot, itd imterholecular cobereicb afíer vimyLRd. */

- *

- 1. R.e. macfarlame, mew tgermal meUtroy scatterimg files fok */

- EadF/B-VI RELEASE 2, los alamos mitiomal laboratory report */

* (TO BE PUBLISBED). *

* *

I

STOP

Figures 94 and 95 show the frequency spectrum for the hindered rotations and the static structure factor, respectively.

Several views of the scattering law for liquid para deuterium are shown in Figures 96 through 99 . The asymmetric version of $S(\alpha, \beta)$ is plotted, because liquid deuterium does not obey the microscopic reversibility condition required for having a symmetric scattering law. Note that the $\alpha$ and $\beta$ grids are sufficiently detailed to represent the important features. The cusps evident in Figure 99 are due to plotting semi-log curves on a log-log plot. Similarly, Figures 100 through 103 show the scattering law for liquid ortbo deuterium.

Figure 104 shows the cross sections for para and ortho deuterium and compares them to experimental data for the equilibrium mixture at $19 \mathrm{~K}(1 / 3$ para, $2 / 3$ ortho). Figures 105 and 106 show outgoing neutron spectra for three different values of the incident energy. 


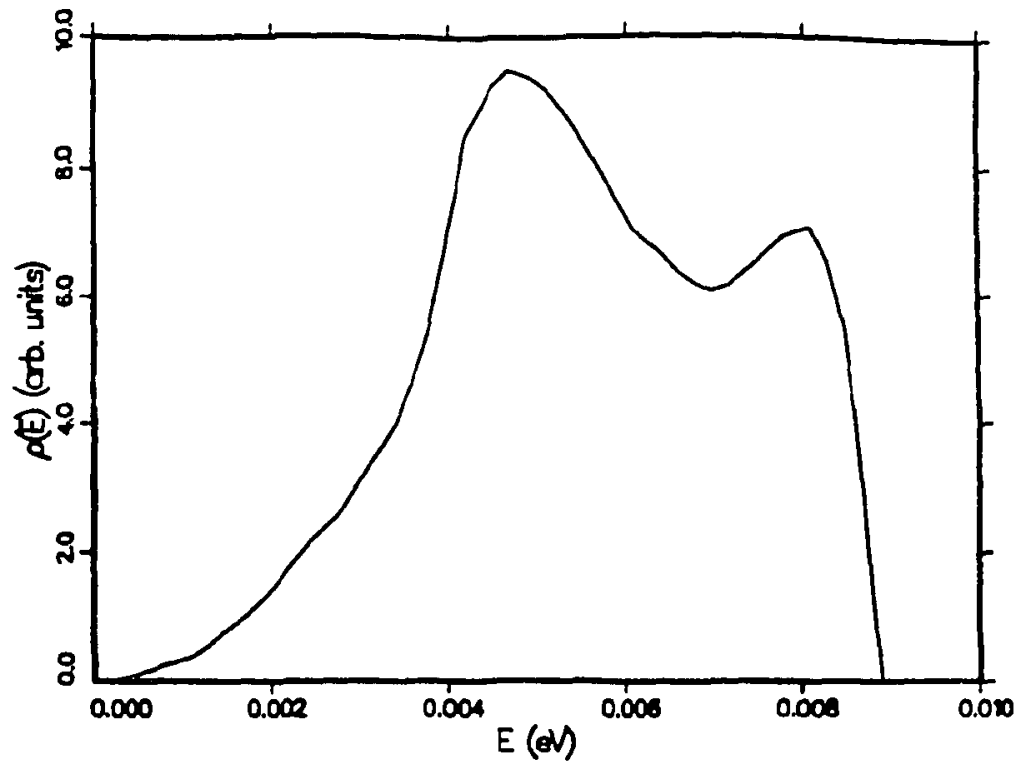

Figure 94: The Keinert-Sax frequency distribution for the effective translational modes of liquid deuterium.

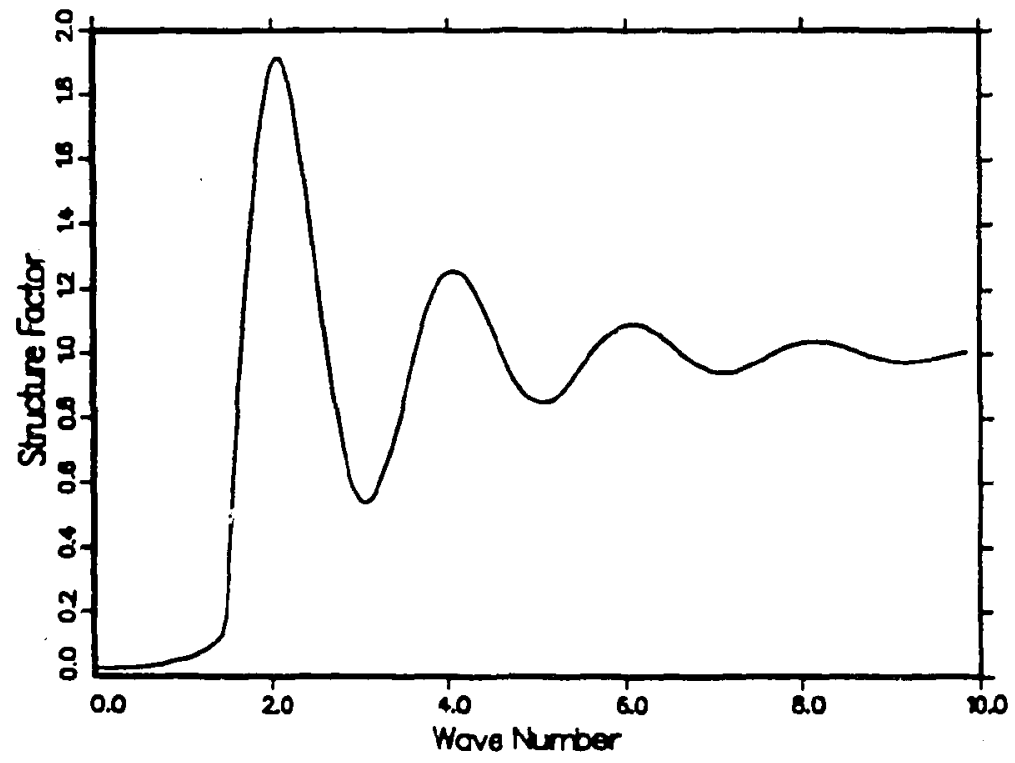

Figure 95: The static structure factor $S(\kappa)$ for liquid cleuterium. 


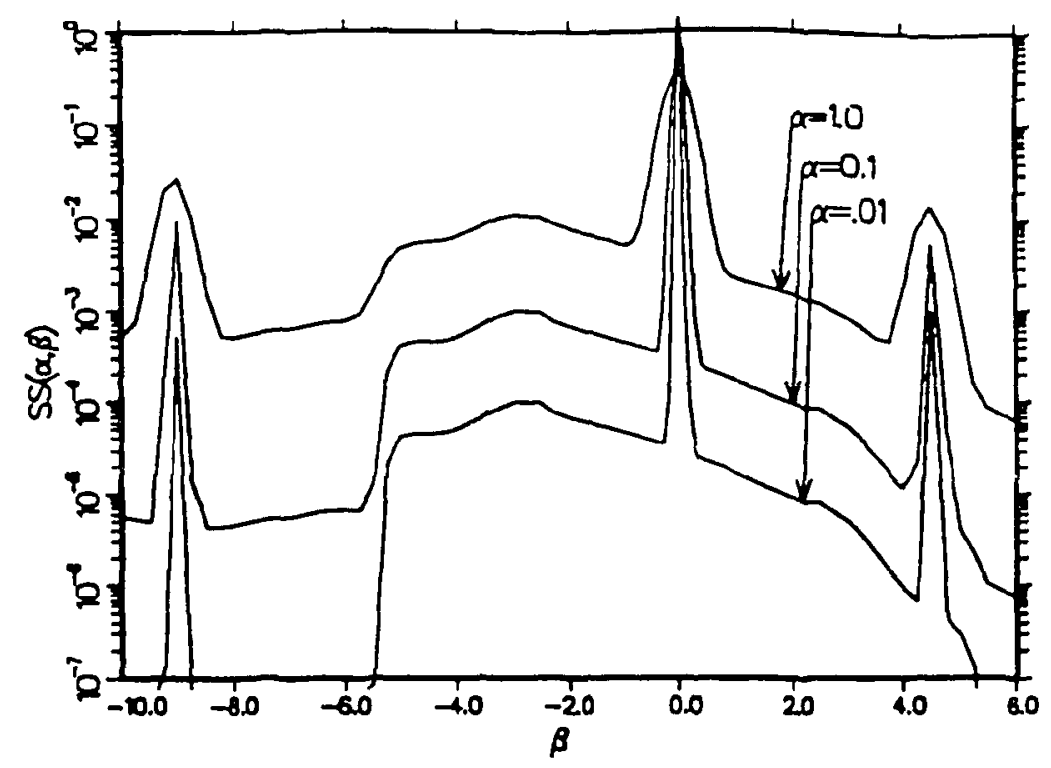

Figure 96: Script-S for liquid para deuterium at $19 \mathrm{~K}$ is shown as a function of $\beta$ for several $\alpha$ values, concentrating on the low- $\alpha$ and low- $\beta$ range.

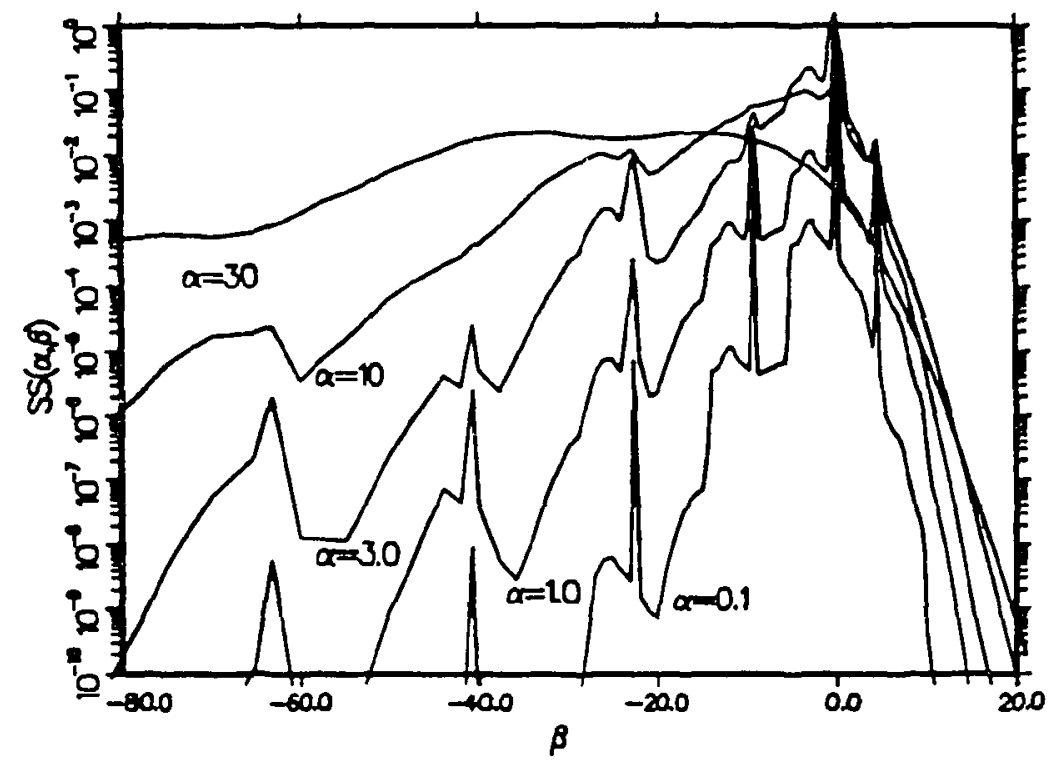

Figure 97: Script-S for liquid para deuterium at $19 \mathrm{~K}$ is shown as a function of $\beta$ for several $\alpha$ values, concentrating on the middle range of $\alpha$ and $\beta$ values. 


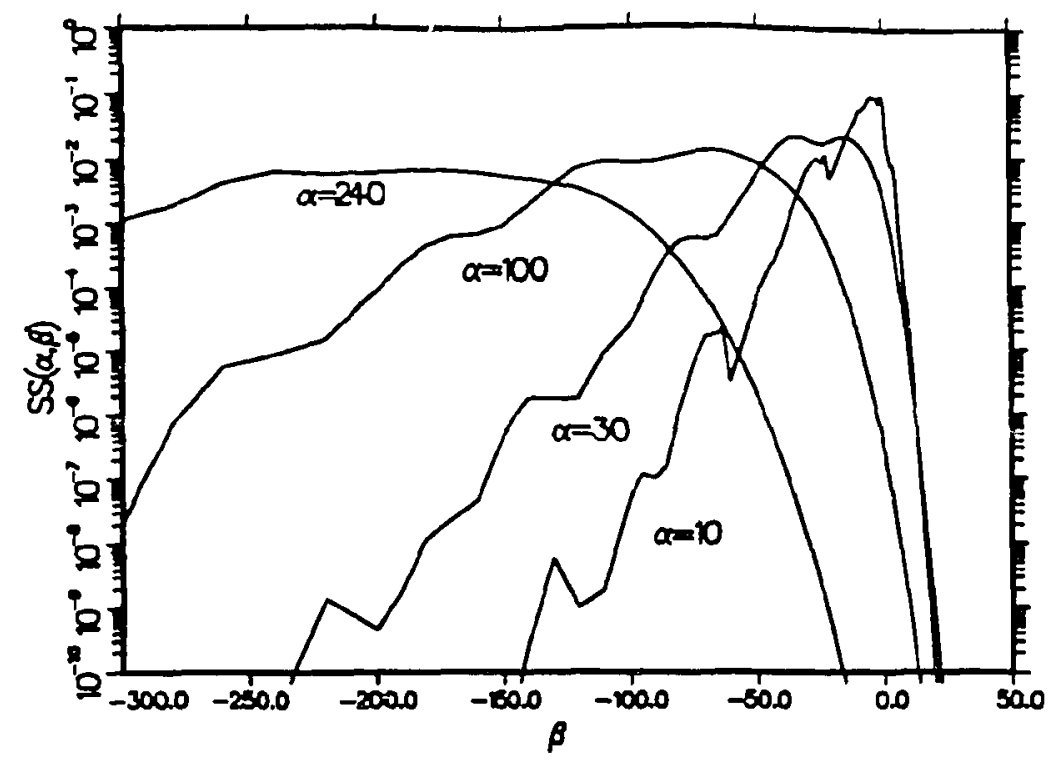

Figure 98: Script-S for liquid para deuterium at $19 \mathrm{~K}$ is shown as a function of $\beta$ for several $\alpha$ values, concentrating on bigh $\alpha$ and $\beta$ values.

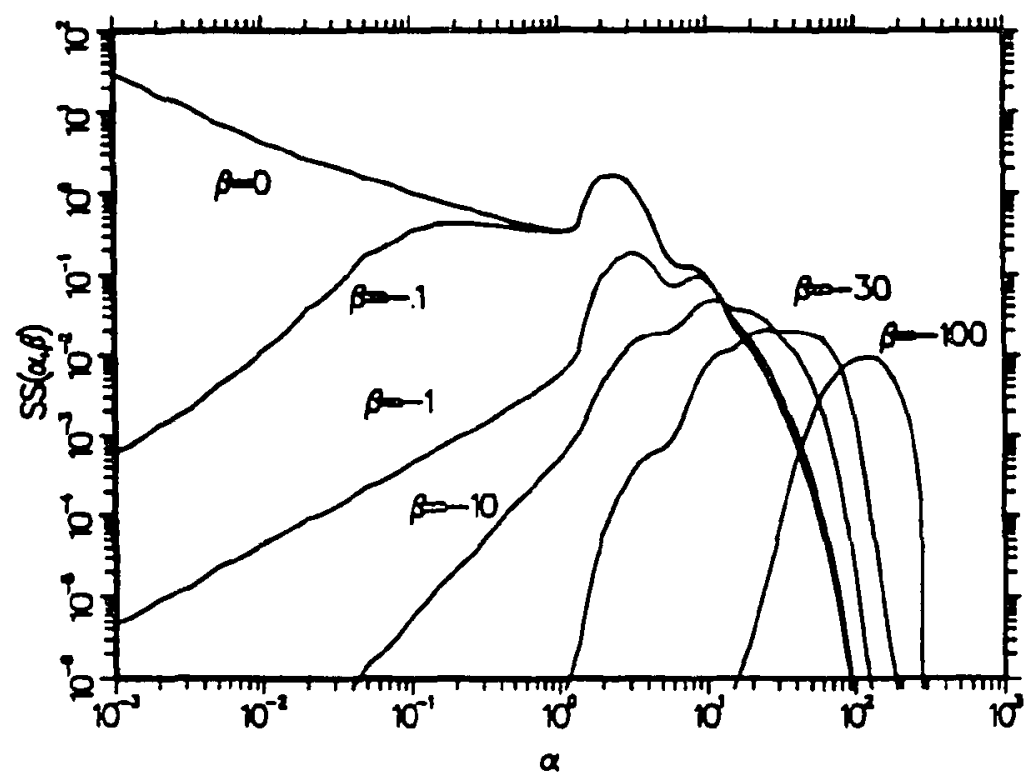

Figure 99: Script-S for liquid para deuterium at $19 \mathrm{~K}$ is shown as a function of $\alpha$ for several $\beta$ values corresponding to downscatter. 


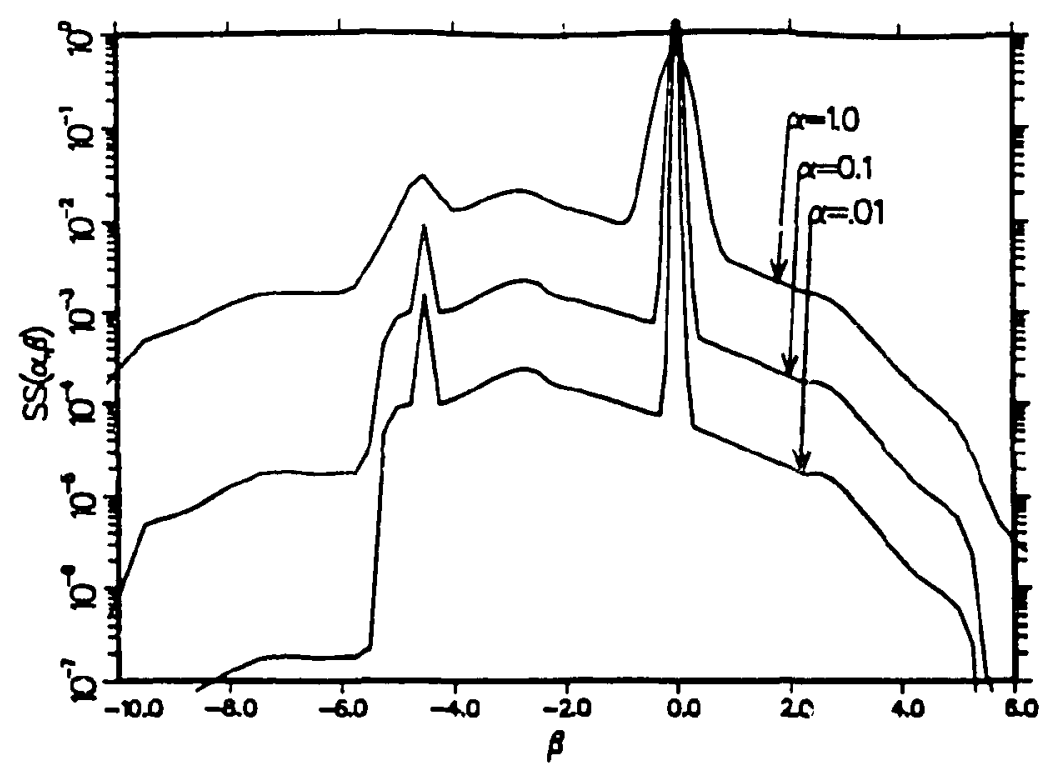

Figure 100: Script-S for liquid ortho deuterium at $19 \mathrm{~K}$ is shown as a function of $\beta$ for several $\alpha$ values, concentrating on the low- $\alpha$ and low- $\beta$ range.

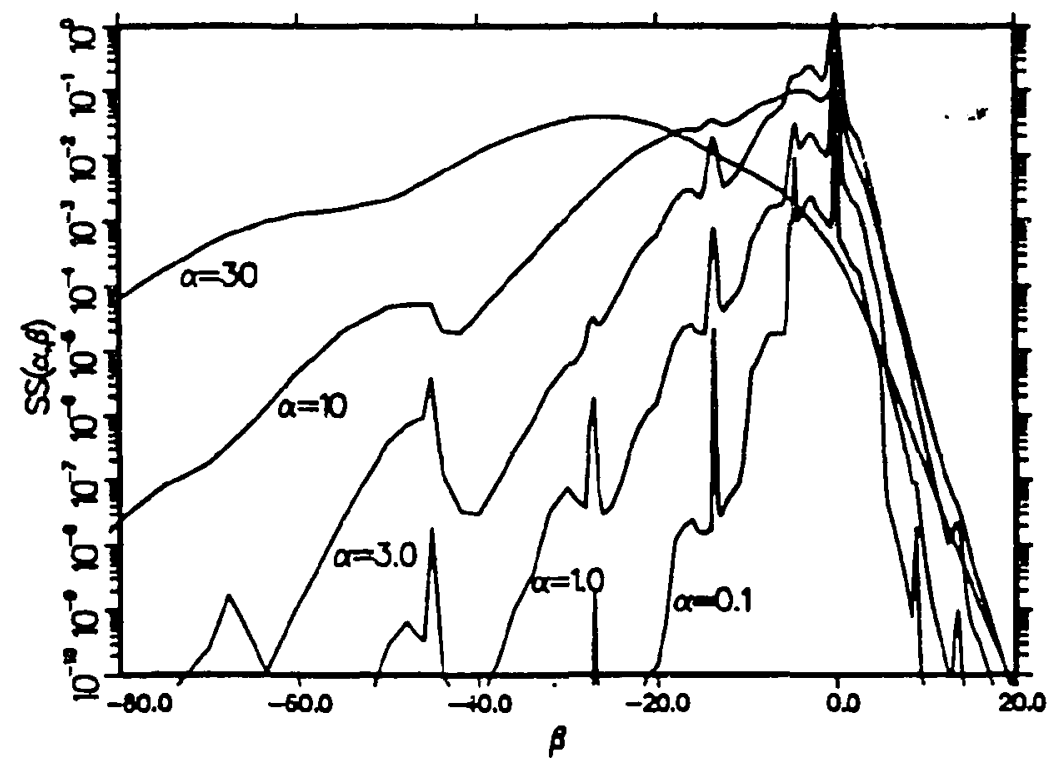

Figure 101: Script-S for liquid ortho deuterium at $19 \mathrm{~K}$ is shown as a function of $\beta$ for several $\alpha$ values, concentrating on the middle range of $\alpha$ and $\beta$ values. 


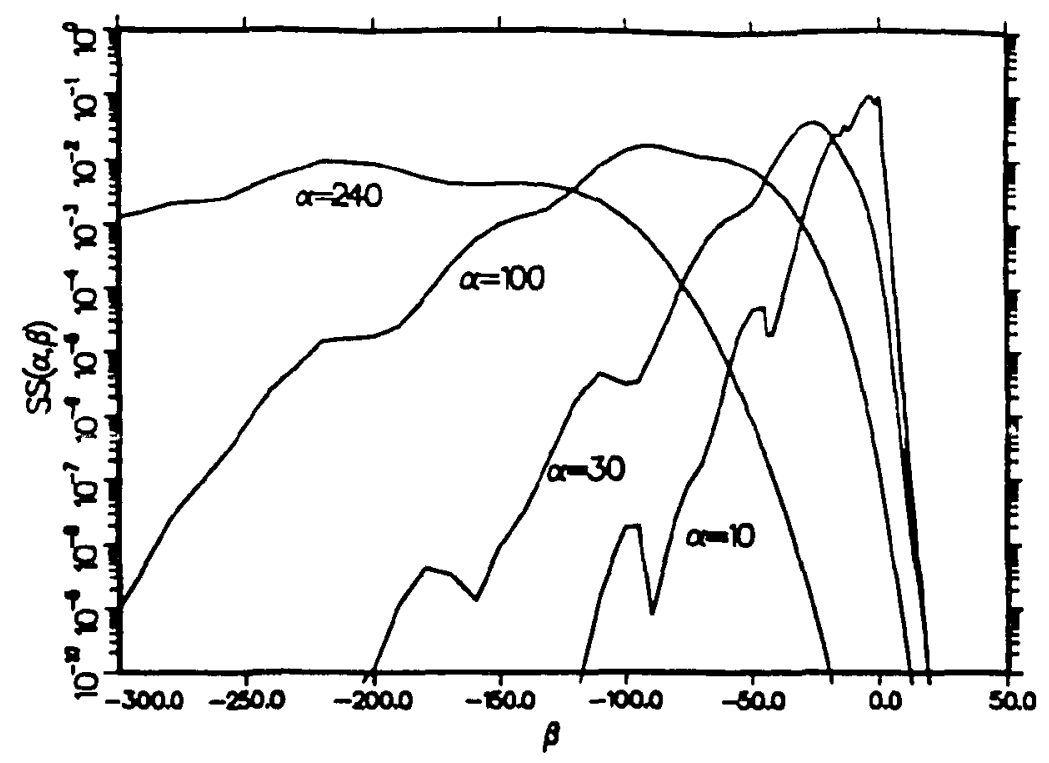

Figure 102: Script-S for liquid ortho deuterium at $19 \mathrm{~K}$ is shown as a function of $\beta$ for several $\alpha$ values, concentrating on high $\alpha$ and $\beta$ values.

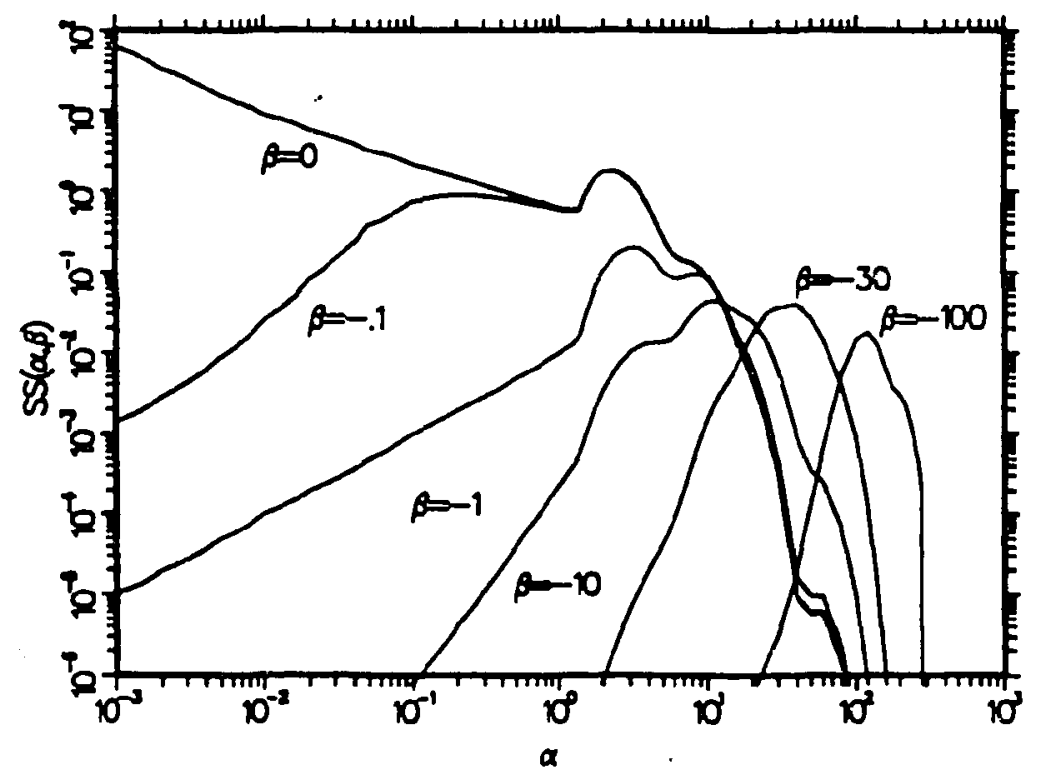

Figure 103: Script-S for liquid ortho deuterium at $19 \mathrm{~K}$ is shown as a function of $\alpha$ for several $\beta$ values corresponding to downscatter. 


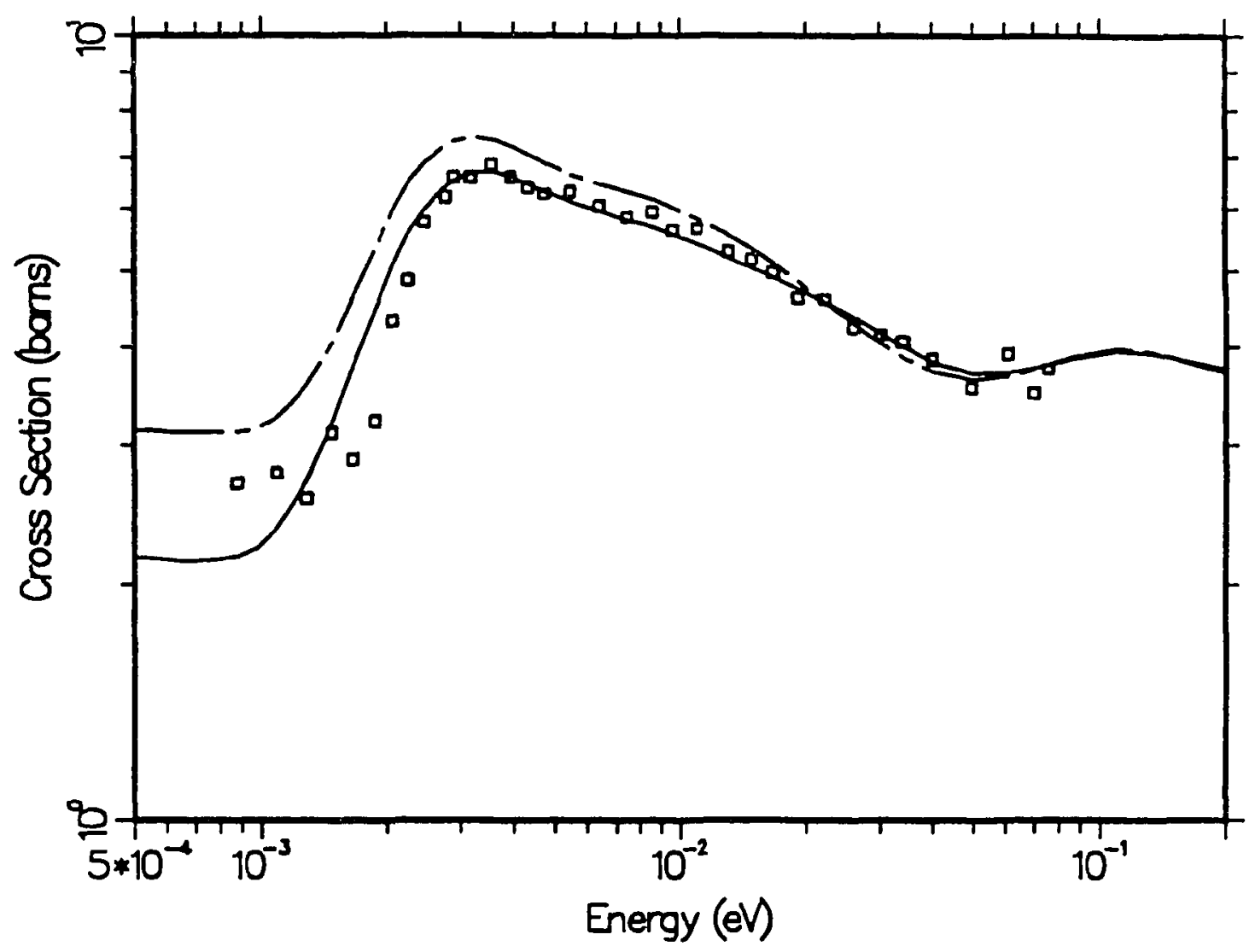

Figure 104: The cross sections for liquid para deuterium (solid curve) and liquid ortho deuterium (chain-dash curve) at $19 \mathrm{~K}$ are compared with experimental data of Seiffert for an equilibrium ortho-para mixture at $19 \mathrm{~K}$ (se Ref. 30). The drop in the cross sections below $.003 \mathrm{eV}$ is due to intermolecular interference. 


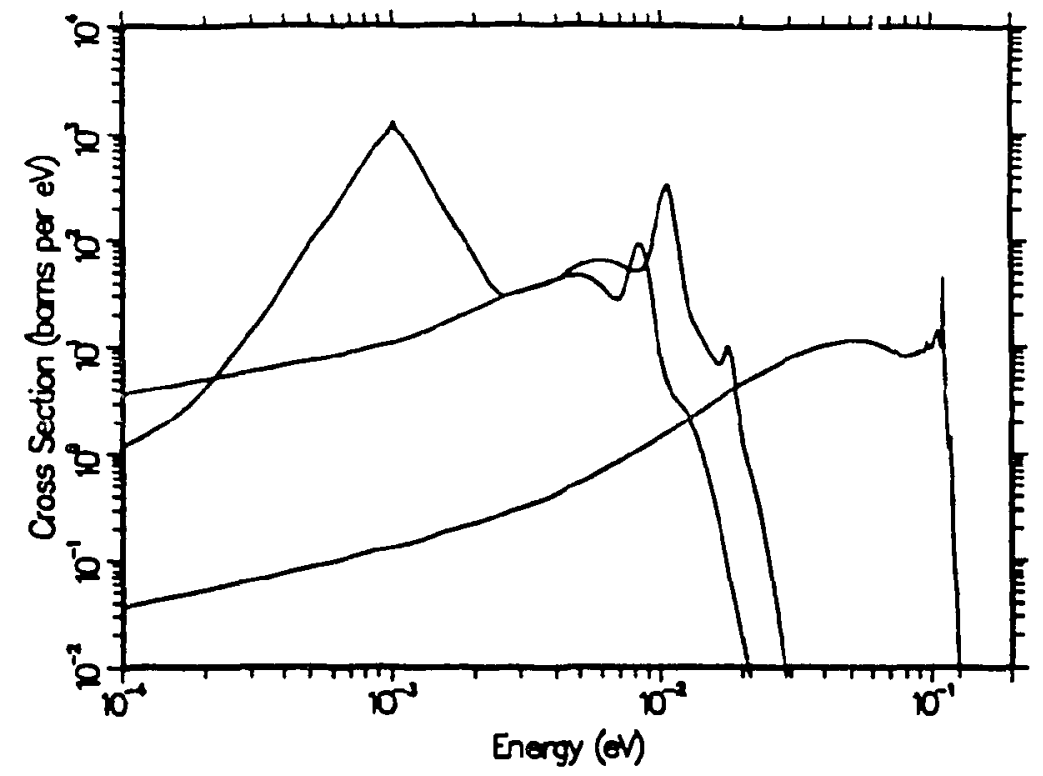

Figure 105: The neutron emission spectra for liquid para deuterium at $19 \mathrm{~K}$ are shown for incident energies of $0.001,0.0106$, and $0.112 \mathrm{eV}$.

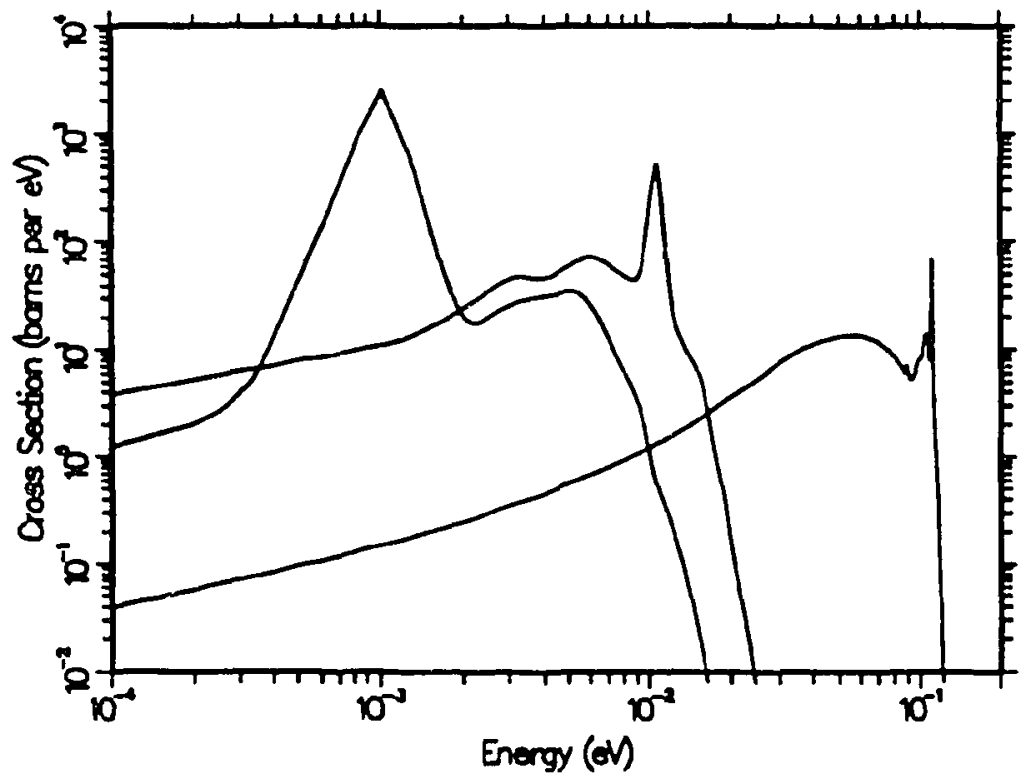

Figure 108: The neutron emission spectra for liquid ortho deuterium at $19 \mathrm{~K}$ are shown for incident energies of $0.001,0.0106$, and $0.112 \mathrm{eV}$. 
Alamos, New Mexico, Los Alamos National Laboratory report LA-121.46-C (August 1991).

7. J. U. Koppel, J. R. Triplett, and Y. D. Naliboff, "GASKET: A Unified Code for Thermal Neutron Scattering," General Atomics report GA-7417 (Rev.) (March 1967).

8. D. E. Parks, M S. Nelkin, J. R. Beyster, and N. F. Wikner, Slow Neutron Scattering and Thermalization, W. A. Benjamin, Inc. (New York, 1970).

9. Y. D. Naliboff and J. U. Koppel, "Coherent Elastic Scattering of Neutrons by Hexagonal Lattices," General Atomic report GA-6026(1964).

10. R. W. G. Wycoff, Crystal Structures, Interscience Publishers (New York, 1960).

11. James A. Young and Juan U. Koppel, Phys. Rev. 135, A603 (1964).

12. P. A. Egelstaff, B. C. Haywood, and F. J. Webb, Proc. Phys. Soc., 90, 681 (1967).

13. Wolfgarig Schott, Z. Physik 231, 243 (1970).

14. Masahiko Utsuro, Z. Physik B 27, 111 (1977).

15. J. Keinert and J. Sax, "Investigation of Neutron Scattering Dynamics in Liquid Hydrogen and Deuterium for Cold Neutron Sources," Kerntechnik 51, 19 (1987).

16. George. H. Vineyard, Phys. Rev. $\underline{110}, 999$ (1958).

17. R. E. Schmunk, R. M. Brugger, F. ר. Randolph, and K. A. Strong, Phys. Rev. 128, 562 (1962).

18. G. Borgonovi, "Lattice Dynamics and Neutron Scattering of BeO," General Atomic report GA-8758 (1968).

19. R. M. Brugger, K. A. Strong, and J. M. Carpenter, J. Phys. Chem. Solids 28, 249 (1967).

20. J. A. Young, N. F. Wilkner, and D. E. Parks, Nukleonik band 1, 295(1965).

21. J. A. Young and J. U. Koppel, J. Chem. Phys. 42, 357 (1965).

22. E. L. Slaggie, "Central Force Lattice Dynamical Model for Zirconium Hydride," General Atomic report GA-8132 (July 1967).

23. Y. D. Harker and R. M. Brugger, "Investigation of the Low-Temperature Phase Transitions in Solid Methane by Slow Neutron Inelastic Scattering," J. Chem. Phys. 46, 2201 (1967).

24. Ashck K. Agrawal and Sidney Yip, "Slow-Neutron Scattering by Molecular Liquids," Nucl. Sci. Eng. 37, 368 (1969).

25. R. G. Gordon, J. Chem. Phys. $\underline{43}, 1307$ (1965).

26. G. E. Ewing, J. Chem. Phys. 40, 179 (1964). 
27. These data were obtained from the paper of Keinert and $\operatorname{Sax}^{27}$ and converted to the numbers shown by subtracting the hydrogen absorbtion cross section. The ortho cross sections were converted from normal $\mathrm{H}_{2}$ cross sections using the theoretical ortho and para cross sections to produce a conversion factor. The original references are G. L. Squires and A. T. Stewart, Proc. Roy. Soc. A230, 19 (1955), W. L. Whittemore and A. W. McReynolds, "Differential Neutron Thermalization," General Atomic report GA-2505 (1961), and W. D. Seiffert, "Messung der Streuquerschnitte von flüssigem und festem Wasserstoff, Deuterium, und Deuteriumhydrid für thermische Neutronen," EUR-5566d (1970).

28. These data were obtained from graphs provided by Keinert and Sax as a private communication. The original source was W. D. Seiffert, "Messung der Streuquerschnitte von flüssigem und festem Wasserstoff, Deuterium, und Deuteriumhydrid für thermische Neutronen," EUR-5566d (1970). 